\title{
Cobalt-Catalyzed Enantioselective Vinylation of Activated Ketones and Imines
}

\author{
Yuan Huang, Rui-Zhi Huang, Yu Zhao* \\ Department of Chemistry, National University of Singapore \\ 3 Science Drive 3, Singapore 117543 \\ Email: zhaoyu@nus.edu.sg
}

\section{Supporting Information}

\section{Contents:}

1. General information 2

2. General procedure for cobalt-catalyzed enantioselective vinylation $\quad 3-4$

3. Analytical data of vinylation products 2

4. Analytical data of vinylation products $7 \quad 19-29$

5. Analytical data of vinylation products $14 \quad 30-38$

6. General procedure of derivatization and analytical data 39-43

7. NMR spectra of the products and derivatives 44-104

8. X-ray analysis of compounds ent-2g, 3 and 14k 105-144

9. Reference 145 


\section{General Information}

Chemicals and solvents were purchased from commercial suppliers and used as received. ${ }^{1} \mathbf{H}$ and ${ }^{13} \mathbf{C}$ NMR spectra were recorded on a Bruker ACF300 (300 MHz) or AMX500 (500 MHz) spectrometer. Chemical shifts were reported in parts per million (ppm), and the residual solvent peak was used as an internal reference: proton (chloroform $\delta$ 7.26), carbon (chloroform $\delta 77.0$ ) or tetramethylsilane (TMS $\delta 0.00$ ) was used as a reference. Multiplicity was indicated as follows: $\mathrm{s}$ (singlet), $\mathrm{d}$ (doublet), $\mathrm{t}$ (triplet), q (quartet), m (multiplet), dd (doublet of doublet), bs (broad singlet). Coupling constants were reported in Hertz (Hz). Low resolution mass spectra were obtained on a Finnigan/MAT LCQ spectrometer in ESI mode, and a Finnigan/MAT 95XL-T mass spectrometer in EI mode. All high resolution mass spectra (HRMS) were obtained on a Finnigan/MAT 95XL-T spectrometer. For thin layer chromatography (TLC), Merck pre-coated TLC plates (Merck 60 F254) were used, and compounds were visualized with a UV light at $254 \mathrm{~nm}$. Further visualization was achieved by staining with iodine, or potassium permanganate solution followed by heating using a heat gun. Flash chromatography separations were performed on Merck 60 (0.040-0.063 mm) mesh silica gel. The enantiomeric ratios (er) of products were determined by chiral phase HPLC analysis on SHIMAZU HPLC units, including the following instruments: pump, LC-20AD; detector, SPD-20A; column, Chiralcel OD-H, Chiralpak AS-H, IC, IE or AD-H. Optical rotations were recorded on an MRC AP81 automatic polarimeter.

All reactions were carried out under nitrogen atmosphere. All commercially available reagents listed below were used as received for the reactions without any purification. THF was dried on alumina columns using a solvent dispensing system. Cyclic imines were synthesized according to the literature. ${ }^{1}$

Commercially available reagents: Vinyl boronic acid, $\mathrm{CoCl}_{2}, \mathrm{CoBr}_{2}, \mathrm{CoI}_{2}, \mathrm{CoF}_{2}$ (Sigma-Aldrich); Chiral phosphine ligands: $(S, S)$-BDPP(Sigma-Aldrich), $(R)$-BINAP (Sigma-Aldrich), (R)-(S)-Josiphos (Strem). 


\section{General Procedure for Cobalt-Catalyzed Enantioselective Vinylation}

1) cobalt-catalyzed asymmetric vinylation of $\alpha$-ketoesters

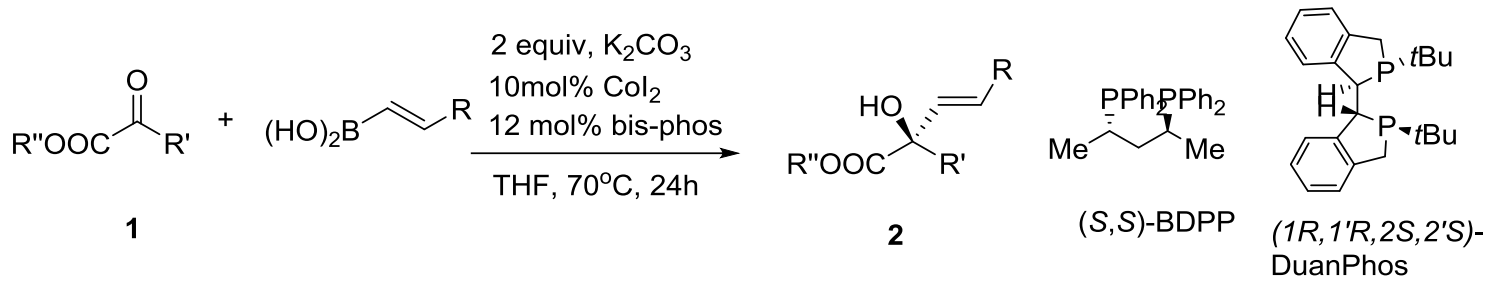

To a $10 \mathrm{~mL}$ vial equipped with a dried stir bar was added cobalt iodide $(0.010 \mathrm{mmol}),(S$, $S$,)-BDPP (for $\mathrm{R}^{\prime}=\mathrm{Me}$ and alkyl) or $\left(1 R, 1^{\prime} R, 2 S, 2^{\prime} S\right)$-DuanPhos (for $\mathrm{R}^{\prime}=$ alkenyl) $(0.012$ mmol) and anhydrous THF (1 mL) in the glovebox. The reaction mixture was then allowed to stir for $30 \mathrm{~min}$, followed by the addition of vinylboronic acid $(0.15 \mathrm{mmol}), \alpha$-ketoesters 1 $(0.10 \mathrm{mmol})$ and 2 equiv. $\mathrm{K}_{2} \mathrm{CO}_{3}$. The reaction mixture was taken outside the glovebox and allowed to stir at $70{ }^{\circ} \mathrm{C}$ for $24 \mathrm{~h}$. The crude reaction mixture was concentrated under reduced pressure. The products $\mathbf{2}$ were directly purified by silica gel chromatography (ethyl acetate:hexanes =1: 9). The enantiopurity of the purified products was analyzed by chiral HPLC (Chiralcel OD-H; OJ-H, AS-H, AD-H).

2) cobalt-catalyzed enantioselective vinylation of isatins<smiles>[R2]N1C(=O)C(=O)c2cc[R17]cc21</smiles>

6

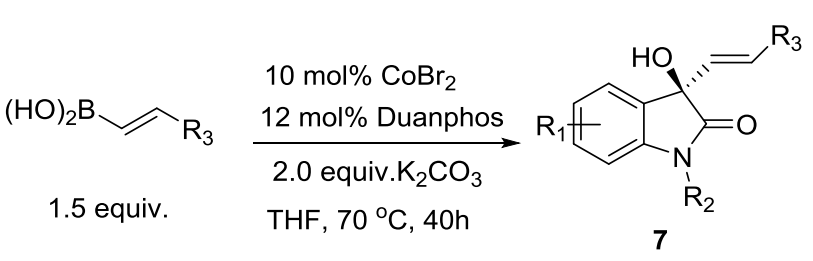

7

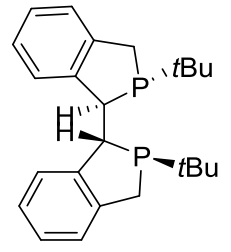

$\left(1 R, 1^{\prime} R, 2 S, 2^{\prime} S\right)-$ DuanPhos

To a $10 \mathrm{~mL}$ vial equipped with a dried stir bar was added cobalt bromide $(0.010 \mathrm{mmol})$, $\left(1 R, 1^{\prime} R, 2 S, 2^{\prime} S\right)$-DuanPhos $(0.012 \mathrm{mmol})$ and anhydrous THF $(1 \mathrm{~mL})$ in the glovebox. The reaction mixture was then allowed to stir for $30 \mathrm{~min}$, followed by the addition of vinylboronic 
acid $(0.15 \mathrm{mmol})$, isatin $6(0.10 \mathrm{mmol})$ and 2 equiv. $\mathrm{K}_{2} \mathrm{CO}_{3}$. The reaction mixture was taken outside the glovebox and allowed to stir at $70{ }^{\circ} \mathrm{C}$ for $40 \mathrm{~h}$. The crude reaction mixture was concentrated under reduced pressure. The products 7 were directly purified by silica gel chromatography (ethyl acetate:DCM = 1: 5). The enantiopurity of the purified products was analyzed by chiral HPLC (Chiralcel OD-H; AS-H, AD-H).

\section{3) cobalt-catalyzed asymmetric vinylation of imines}<smiles></smiles>

14

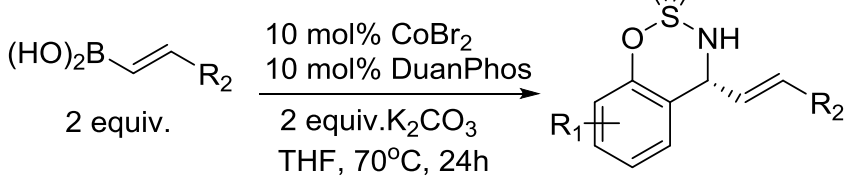

16

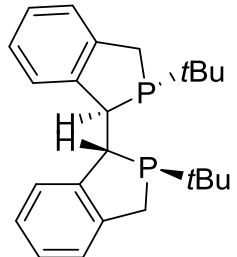

$\left(1 R, 1^{\prime} R, 2 S, 2^{\prime} S\right)$-DuanPhos

To a $10 \mathrm{~mL}$ vial equipped with a dried stir bar was added cobalt bromide $(0.010 \mathrm{mmol})$, $\left(1 R, 1^{\prime} R, 2 S, 2^{\prime} S\right)$-DuanPhos $(0.010 \mathrm{mmol})$ and anhydrous THF $(0.5 \mathrm{~mL})$ in the glovebox. The reaction mixture was then allowed to stir for $30 \mathrm{~min}$, followed by addition of vinylboronic acid $(0.20 \mathrm{mmol})$, imines $14(0.10 \mathrm{mmol})$ and 2 equiv. $\mathrm{K}_{2} \mathrm{CO}_{3}$. The reaction mixture was taken outside the glovebox and allowed to stir at $70{ }^{\circ} \mathrm{C}$ for $24 \mathrm{~h}$. The crude reaction mixture was concentrated under reduced pressure. The products $\mathbf{1 6}$ were directly purified by silica gel chromatography (ethyl acetate:hexanes =1:5). The enantiopurity of the purified products was analyzed by chiral HPLC (Chiralcel OD-H; Chiralpak AS-H, AD-H, IE). 


\section{Analytical data of vinylation products 2}

\section{Ethyl $(R, E)$-2-hydroxy-2-methyl-4-phenylbut-3-enoate}

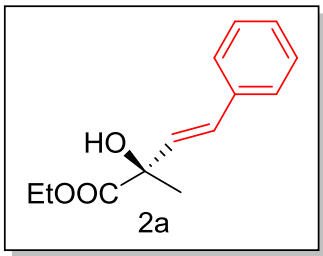

Colorless oil, $60 \%$ yield.

${ }^{1} \mathbf{H}$ NMR $\left(300 \mathrm{MHz}, \mathrm{CDCl}_{3}\right): \delta 7.46-7.25(\mathrm{~m}, 5 \mathrm{H}), 6.85(\mathrm{~d}, J=15.9$ $\mathrm{Hz}, 1 \mathrm{H}), 6.38(\mathrm{~d}, J=15.9 \mathrm{~Hz}, 1 \mathrm{H}), 4.45-4.18(\mathrm{~m}, 2 \mathrm{H}), 3.52(\mathrm{~s}, 1 \mathrm{H})$, $1.63(\mathrm{~s}, 3 \mathrm{H}), 1.36(\mathrm{t}, J=7.1 \mathrm{~Hz}, 3 \mathrm{H})$.

${ }^{13} \mathrm{C}$ NMR $\left(75 \mathrm{MHz}, \mathrm{CDCl}_{3}\right): \delta 175.52,136.27,130.78,129.32$, $128.50,127.74,126.62,74.41,62.31,26.23,14.08$.

HRMS (ESI): m/z Calcd. for $\left[\mathrm{C}_{13} \mathrm{H}_{16} \mathrm{NaO}_{3}, \mathrm{M}+\mathrm{Na}\right]^{+}:$243.0997; Found: 243.0988 .

Optical Rotation: $[\alpha]_{D}^{24}=-56.83\left(c=0.17, \mathrm{CHCl}_{3}\right)$, The absolute configuration was assigned by the X-ray crystallography of the hydrolysis product 3 .

96:4 er. HPLC condition: Chiralcel OJ-H, $n$-hexane $/ i-\mathrm{PrOH}=99: 1$, flow rate $=1 \mathrm{ml} / \mathrm{min}$, wavelength $=254 \mathrm{~nm}, \mathrm{t}_{\mathrm{R}}=18.443 \mathrm{~min}$ for minor isomer, $\mathrm{t}_{\mathrm{R}}=21.197$ min for major isomer.
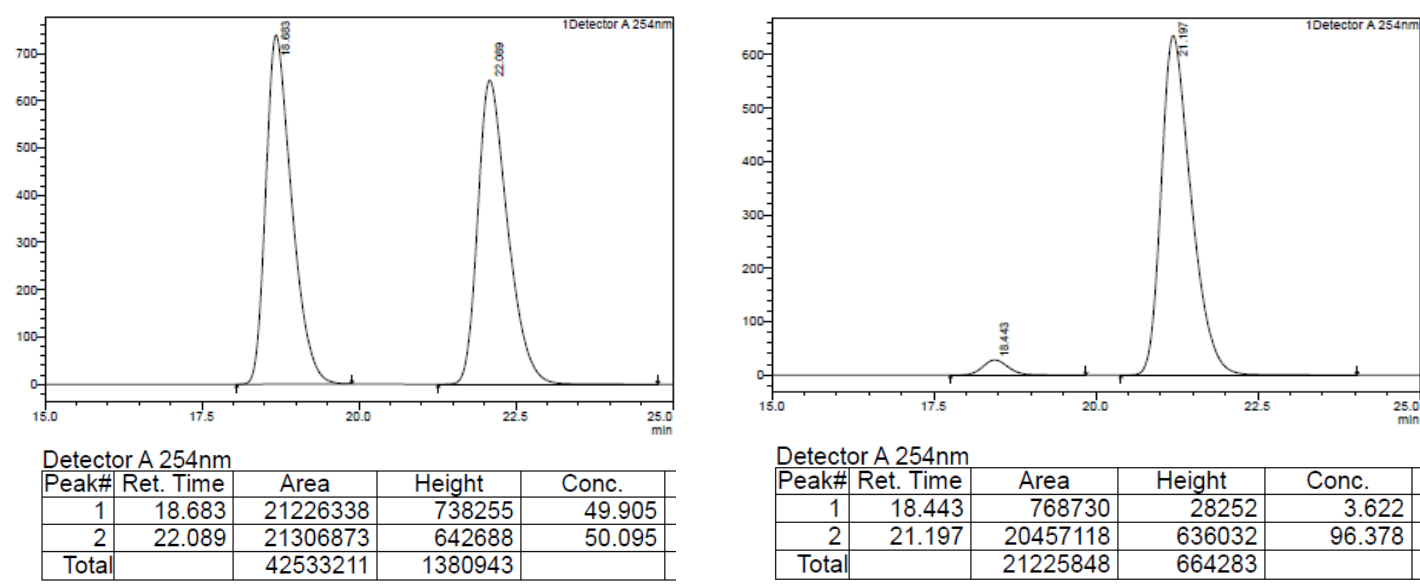

Ethyl (R, E)-4-(4-fluorophenyl)-2-hydroxy-2-methylbut-3-enoate

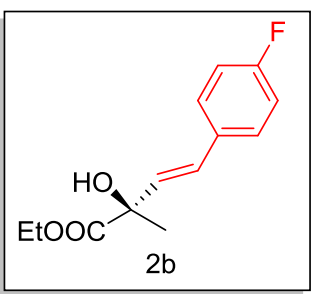

Colorless oil, $60 \%$ yield.

${ }^{1} \mathbf{H}$ NMR $\left(500 \mathrm{MHz}, \mathrm{CDCl}_{3}\right): \delta 7.38(\mathrm{dd}, J=8.6,5.5 \mathrm{~Hz}, 2 \mathrm{H}), 7.03(\mathrm{t}$, $J=8.7 \mathrm{~Hz}, 2 \mathrm{H}), 6.79(\mathrm{~d}, J=15.9 \mathrm{~Hz}, 1 \mathrm{H}), 6.28(\mathrm{~d}, J=15.9 \mathrm{~Hz}, 1 \mathrm{H})$, $4.40-4.18(\mathrm{~m}, 2 \mathrm{H}), 3.51(\mathrm{~s}, 1 \mathrm{H}), 1.60(\mathrm{~s}, 3 \mathrm{H}), 1.35(\mathrm{t}, J=7.1 \mathrm{~Hz}, 3 \mathrm{H})$. ${ }^{13} \mathrm{C}$ NMR $\left(125 \mathrm{MHz}, \mathrm{CDCl}_{3}\right): \delta 175.54,162.46\left(\mathrm{~d}, J_{F C}=247.2 \mathrm{~Hz}\right)$, $132.55\left(\mathrm{~d}, \mathrm{~J}_{\mathrm{FCCCC}}=3.4 \mathrm{~Hz}\right), 130.62\left(\mathrm{~d}, J_{F C C C C C}=2.2 \mathrm{~Hz}\right), 128.29$, $128.23\left(\mathrm{~d}, J_{F C C C}=8.0 \mathrm{~Hz}\right), 115.48\left(\mathrm{~d}, J_{F C C}=21.6 \mathrm{~Hz}\right), 74.42,62.40,26.36,14.15$.

${ }^{19}$ F NMR $\left(282 \mathrm{MHz}, \mathrm{CDCl}_{3}\right): \delta-114.18(\mathrm{tt}, J=8.6,5.4 \mathrm{~Hz}, 1 \mathrm{~F})$.

HRMS (ESI): $\mathrm{m} / \mathrm{z}$ Calcd. for $\left[\mathrm{C}_{13} \mathrm{H}_{15} \mathrm{FNaO}_{3}, \mathrm{M}+\mathrm{Na}\right]^{+}$: 261.0903 ; Found: 261.0895 . 
Optical Rotation: $[\alpha]_{\mathrm{D}}^{24}=-17.00(\mathrm{c}=0.21, \mathrm{MeOH})$. The absolute configuration was assigned by analogue to $\mathbf{2 a}$.

95.5:4.5 er. HPLC condition: Chiralcel OJ-H, $n$-hexane $/ i-\mathrm{PrOH}=95: 5$, flow rate $=1 \mathrm{ml} / \mathrm{min}$, wavelength $=254 \mathrm{~nm}, \mathrm{t}_{\mathrm{R}}=10.065 \mathrm{~min}$ for minor isomer, $\mathrm{t}_{\mathrm{R}}=12.880 \mathrm{~min}$ for major isomer.
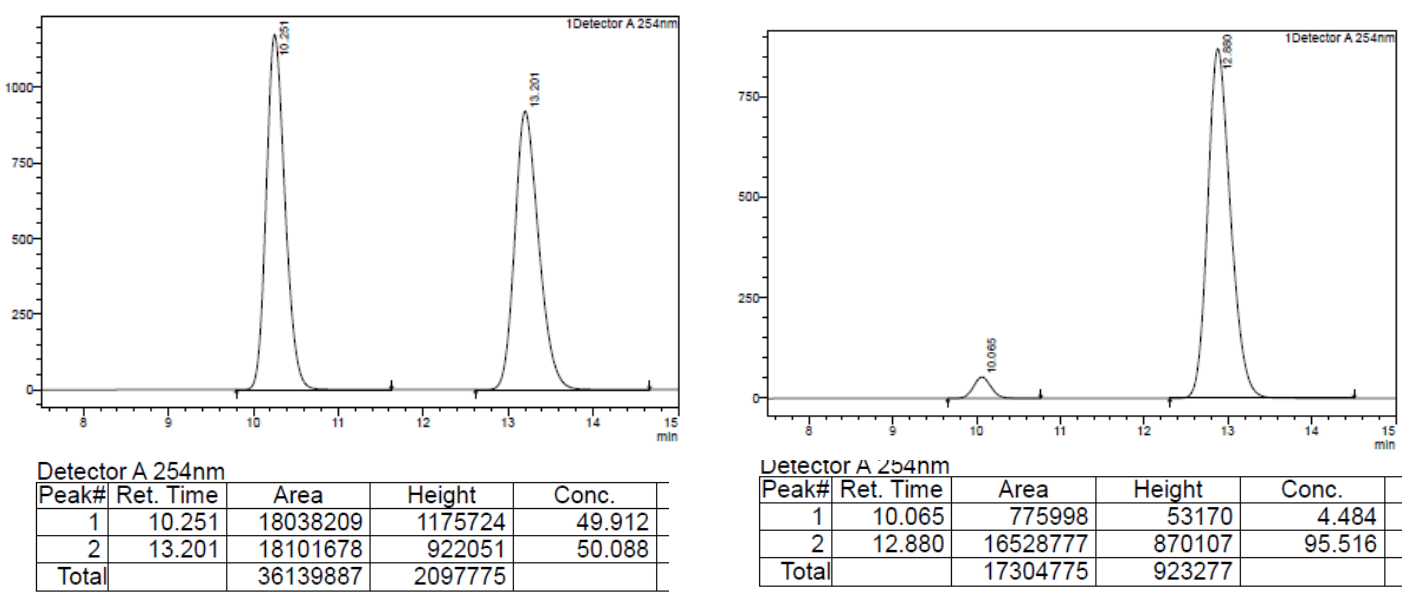

Ethyl $(R, E)$-2-hydroxy-4-(4-methoxyphenyl)-2-methylbut-3-enoate

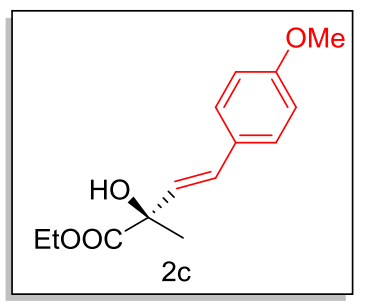

Colorless oil, $75 \%$ yield.

${ }^{1} \mathbf{H}$ NMR $\left(500 \mathrm{MHz}, \mathrm{CDCl}_{3}\right): \delta 7.35(\mathrm{~d}, J=8.7 \mathrm{~Hz}, 2 \mathrm{H}), 6.88(\mathrm{~d}, J$ $=8.6 \mathrm{~Hz}, 2 \mathrm{H}), 6.76(\mathrm{~d}, J=15.9 \mathrm{~Hz}, 1 \mathrm{H}), 6.22(\mathrm{~d}, J=15.8 \mathrm{~Hz}, 1 \mathrm{H})$, $4.41-4.16(\mathrm{~m}, 2 \mathrm{H}), 3.83(\mathrm{~s}, 3 \mathrm{H}), 3.47(\mathrm{~s}, 1 \mathrm{H}), 1.60(\mathrm{~s}, 3 \mathrm{H}), 1.34(\mathrm{t}$, $J=7.1 \mathrm{~Hz}, 3 \mathrm{H})$.

${ }^{13}$ C NMR $\left(125 \mathrm{MHz}, \mathrm{CDCl}_{3}\right): \delta 175.73,159.43,129.14,128.84$, $128.74,127.89,114.02,74.48,62.28,55.30,26.29,14.15$.

HRMS (ESI): $\mathrm{m} / \mathrm{z}$ Calcd. for $\left[\mathrm{C}_{14} \mathrm{H}_{18} \mathrm{NaO}_{4}, \mathrm{M}+\mathrm{Na}\right]^{+}:$273.1103; Found: 273.1095 .

Optical Rotation: $[\alpha]_{D}^{24}=-26.75(c=0.25, \mathrm{MeOH})$, The absolute configuration was assigned by analogue to $\mathbf{2 a}$.

95.5:4.5 er. HPLC condition: Chiralcel OJ-H, $n$-hexane $/ i-\mathrm{PrOH}=95: 5$, flow rate $=1 \mathrm{ml} / \mathrm{min}$, wavelength $=254 \mathrm{~nm}, \mathrm{t}_{\mathrm{R}}=24.337 \mathrm{~min}$ for minor isomer, $\mathrm{t}_{\mathrm{R}}=26.529 \mathrm{~min}$ for major isomer. 

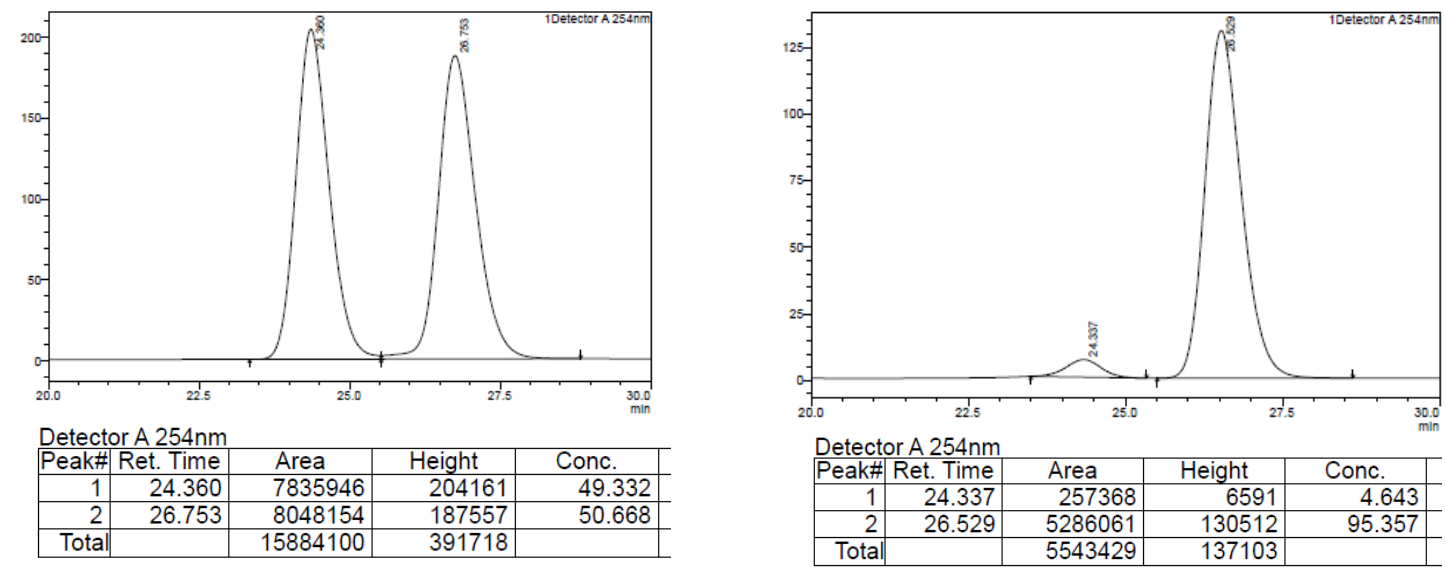

Benzyl $(R, E)$-2-hydroxy-2-methylpent-3-enoate

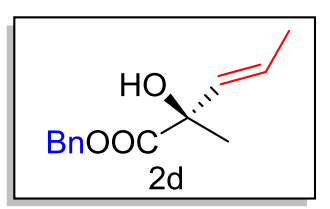

Colorless oil, 74\%, yield.

${ }^{1} \mathbf{H}$ NMR $\left(500 \mathrm{MHz}, \mathrm{CDCl}_{3}\right): \delta 7.44-7.34(\mathrm{~m}, 5 \mathrm{H}), 5.88(\mathrm{dq}, J=15.3$, $6.6 \mathrm{~Hz}, 1 \mathrm{H}), 5.65(\mathrm{dq}, J=15.3,1.6 \mathrm{~Hz}, 1 \mathrm{H}), 5.26(\mathrm{~d}, J=12.3 \mathrm{~Hz}, 1 \mathrm{H})$, $5.20(\mathrm{~d}, J=12.3 \mathrm{~Hz}, 1 \mathrm{H}), 3.25(\mathrm{~s}, 1 \mathrm{H}), 1.72(\mathrm{dd}, J=6.6,1.6 \mathrm{~Hz}, 3 \mathrm{H})$, $1.52(\mathrm{~s}, 3 \mathrm{H})$.

${ }^{13}$ C NMR $\left(125 \mathrm{MHz}, \mathrm{CDCl}_{3}\right): \delta 175.75,135.36,132.54,128.62,128.43,127.97,125.98$, $74.30,67.55,25.82,17.49$.

HRMS (ESI): $\mathrm{m} / \mathrm{z}$ Calcd. for $\left[\mathrm{C}_{13} \mathrm{H}_{16} \mathrm{NaO}_{3}, \mathrm{M}+\mathrm{Na}\right]^{+}:$243.0997; Found: 243.0991 .

Optical Rotation: $[\alpha]_{D}^{24}=11.00(\mathrm{c}=0.14, \mathrm{MeOH})$, The absolute configuration was assigned by analogue to $\mathbf{2 a}$.

94.5:5.5 er. HPLC condition: Chiralcel OJ-H, $n$-hexane $/ i-\mathrm{PrOH}=95: 5$, flow rate $=1 \mathrm{ml} / \mathrm{min}$, wavelength $=227 \mathrm{~nm}, \mathrm{t}_{\mathrm{R}}=19.720 \mathrm{~min}$ for minor isomer, $\mathrm{t}_{\mathrm{R}}=21.617$ min for major isomer.
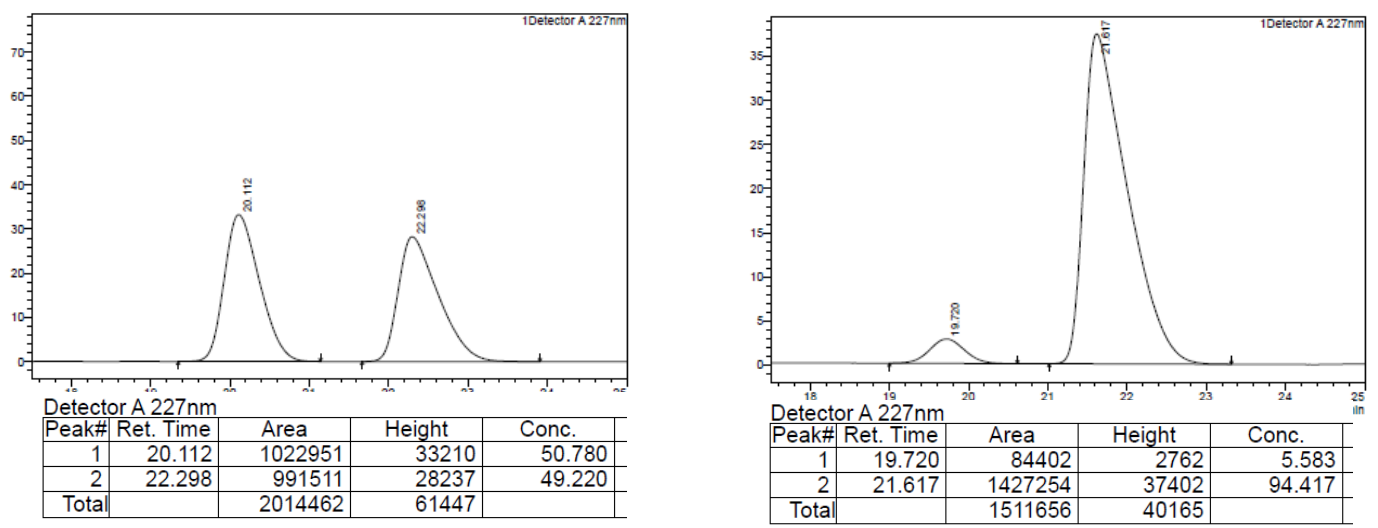


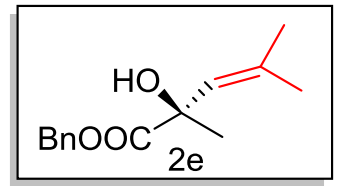

Colorless oil, $30 \%$ yield.

${ }^{1} \mathbf{H}$ NMR $\left(500 \mathrm{MHz}, \mathrm{CDCl}_{3}\right): \delta 7.44-7.31(\mathrm{~m}, 5 \mathrm{H}), 5.38(\mathrm{~d}, J=1.2$ $\mathrm{Hz}, 1 \mathrm{H}), 5.24$ (s, 2H), 1.73 (s, 3H), 1.67 (s, 3H), 1.55 (s, 3H).

${ }^{13} \mathrm{C}$ NMR $\left(75 \mathrm{MHz}, \mathrm{CDCl}_{3}\right): \delta 176.65,138.38,135.38,128.47,128.30$, $127.95,126.02,73.20,67.39,28.19,26.67,18.46$.

HRMS (ESI): $\mathrm{m} / \mathrm{z}$ Calcd. for $\left[\mathrm{C}_{14} \mathrm{H}_{18} \mathrm{NaO}_{3}, \mathrm{M}+\mathrm{Na}\right]^{+}: 257.1154$; Found: 257.1152 .

Optical Rotation: $[\alpha]_{D}^{22}=+38.5(\mathrm{c}=0.14, \mathrm{MeOH})$. The absolute configuration was assigned by analog to that of $2 \mathbf{a}$.

98:2 er. HPLC condition: Chiralpak IE column, $n$-hexane $/ i-\mathrm{PrOH}=95: 5$, flow rate $=1 \mathrm{ml} / \mathrm{min}$, wavelength $=254 \mathrm{~nm}, t_{R}=10.057$ min for minor isomer, $t_{R}=10.550 \min$ for major.
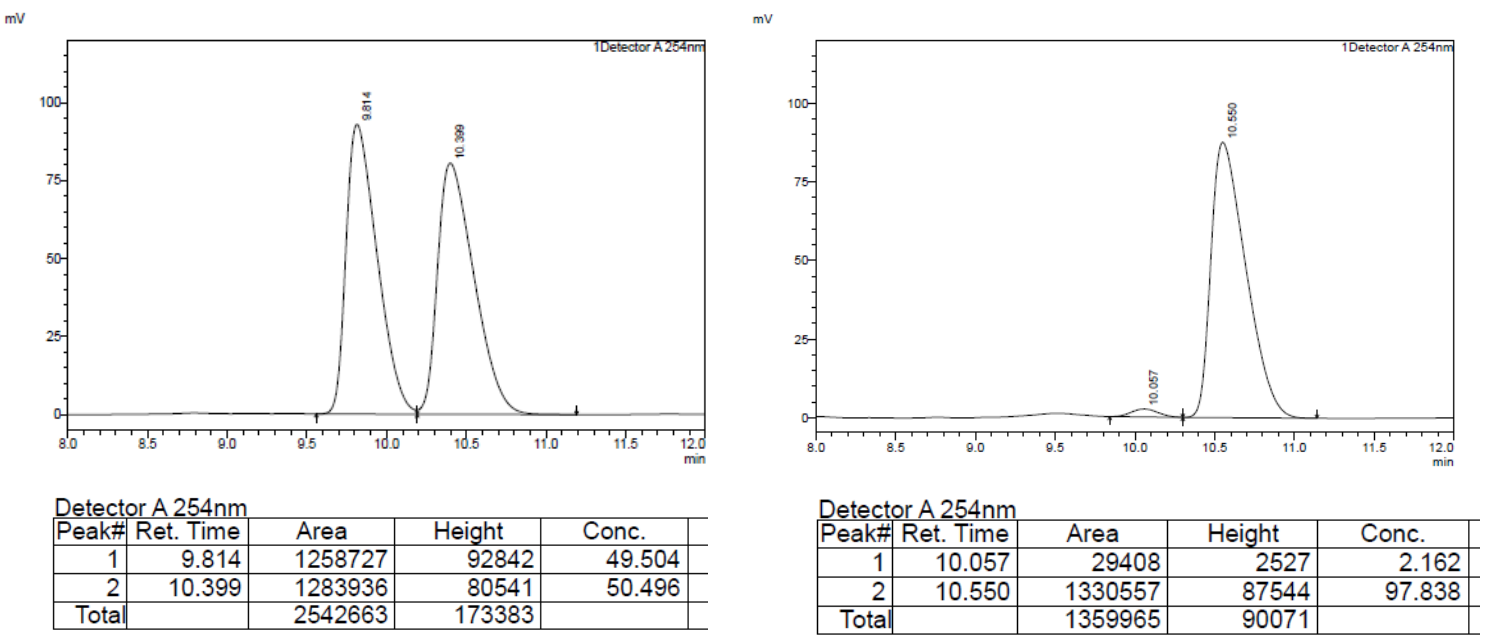

Ethyl $(R, E)$-2-hydroxy-2-phenethyl-4-phenylbut-3-enoate

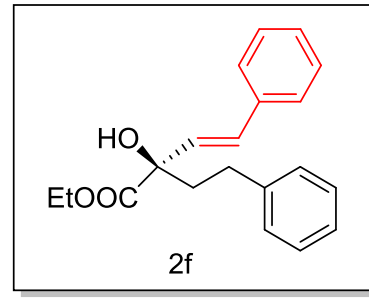

$\mathrm{Hz}, 3 \mathrm{H})$.

${ }^{13} \mathbf{C}$ NMR $\left(125 \mathrm{MHz}, \mathrm{CDCl}_{3}\right): \delta 175.08,141.56,136.39,130.07,129.93,128.57,128.46$, $128.38,127.80,126.70,125.91,77.03,62.47,40.99,29.92,14.20$.

HRMS (ESI): $\mathrm{m} / \mathrm{z}$ Calcd. for $\left[\mathrm{C}_{20} \mathrm{H}_{22} \mathrm{NaO}_{3}, \mathrm{M}+\mathrm{Na}\right]^{+}: 333.1467$; Found: 333.1470

Optical Rotation: $[\alpha]_{\mathrm{D}}^{24}=-9.58\left(\mathrm{c}=0.21, \mathrm{CHCl}_{3}\right)$. The absolute configuration was assigned by analogue to $\mathbf{2 a}$.
Pale yellow oil, $50 \%$ yield.

${ }^{1} \mathbf{H}$ NMR $\left(500 \mathrm{MHz}, \mathrm{CDCl}_{3}\right): \delta 7.43(\mathrm{~d}, J=7.3 \mathrm{~Hz}, 2 \mathrm{H}), 7.38-7.27$ $(\mathrm{m}, 5 \mathrm{H}), 7.25-7.19(\mathrm{~m}, 3 \mathrm{H}), 6.91(\mathrm{~d}, J=15.8 \mathrm{~Hz}, 1 \mathrm{H}), 6.35(\mathrm{~d}, J=$ $15.8 \mathrm{~Hz}, 1 \mathrm{H}), 4.42-4.16(\mathrm{~m}, 2 \mathrm{H}), 2.86(\mathrm{ddd}, J=13.5,11.7,4.9 \mathrm{~Hz}$, $1 \mathrm{H}), 2.61$ (ddd, $J=13.5,11.7,4.9,1 \mathrm{H}), 2.27$ (ddd, $J=13.6,11.6$, $5.2 \mathrm{~Hz}, 1 \mathrm{H}), 2.15(\mathrm{ddd}, J=13.7,11.8,5.0 \mathrm{~Hz}, 1 \mathrm{H}), 1.35(\mathrm{t}, J=7.1$ 
94.5: 5.5 er. HPLC condition: Chiralcel OD-H, $n$-hexane $/ i-\operatorname{PrOH}=99: 1$, flow rate $=1 \mathrm{ml} / \mathrm{min}$, wavelength $=254 \mathrm{~nm}, \mathrm{t}_{\mathrm{R}}=16.002 \min$ for major isomer, $\mathrm{t}_{\mathrm{R}}=18.106$ min for minor isomer.
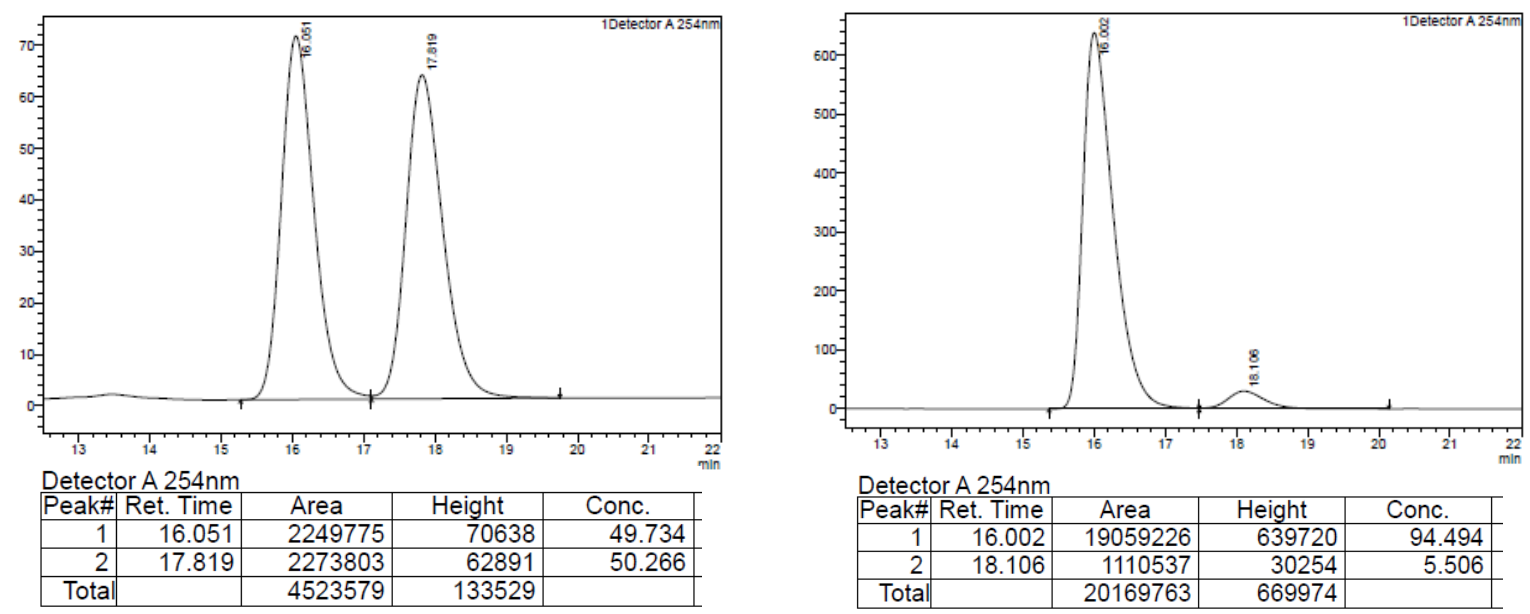

Ethyl $(R, E)$-2-hydroxy-4-phenylbut-3-enoate

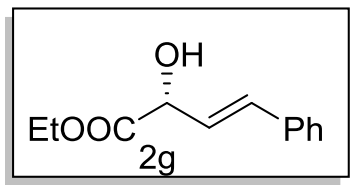

Known Compound, Colorless oil, $70 \%$ yield.

${ }^{1} \mathbf{H}$ NMR $\left(300 \mathrm{MHz}, \mathrm{CDCl}_{3}\right): \delta 7.51-7.15(\mathrm{~m}, 5 \mathrm{H}), 6.82(\mathrm{dd}, J=$ $15.9,1.4 \mathrm{~Hz}, 1 \mathrm{H}), 6.25(\mathrm{dd}, J=15.8,5.5 \mathrm{~Hz}, 1 \mathrm{H}), 4.82(\mathrm{dd}, J=5.5$, $1.6 \mathrm{~Hz}, 1 \mathrm{H}), 4.36-4.22(\mathrm{~m}, 2 \mathrm{H}) 1.32(\mathrm{t}, J=7.1 \mathrm{~Hz}, 3 \mathrm{H})$.

${ }^{13}$ C NMR (75 MHz, $\left.\mathrm{CDCl}_{3}\right): \delta 173.26,136.12,132.07,128.51$, $127.92,126.60,125.41,71.20,62.19,14.08$.

Optical Rotation: $[\alpha]^{23}=-34\left(\mathrm{c}=0.15, \mathrm{CHCl}_{3}\right)$. The absolute configuration was comparing the optical rotation with the reported data. ${ }^{2}$

89.3:10.7 er. HPLC condition: Chiralpak IC column, $n$-hexane $/ i-\mathrm{PrOH}=95: 5$, flow rate $=1$ $\mathrm{ml} / \mathrm{min}$, wavelength $=254 \mathrm{~nm}, \mathrm{t}_{\mathrm{R}}=16.362 \mathrm{~min}$ for minor isomer, $\mathrm{t}_{\mathrm{R}}=19.317$ min for major.
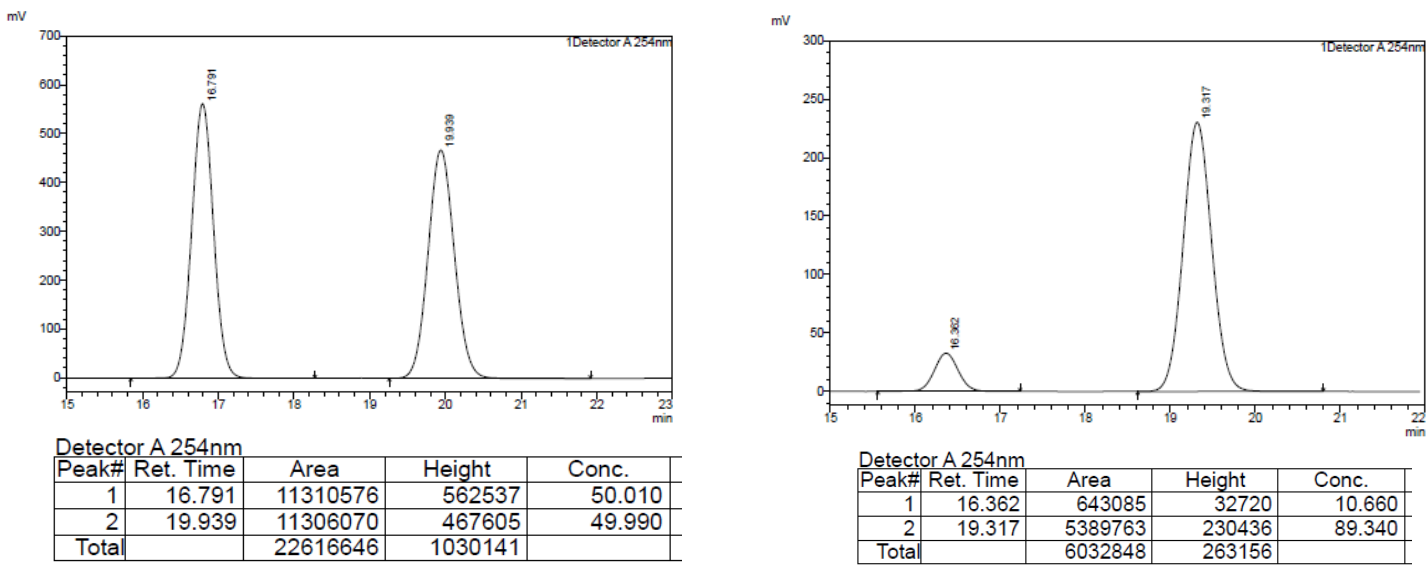
Methyl (R, E)-2-hydroxy-4-(4-methoxyphenyl)-2-((E)-styryl)but-3-enoate (2h)

Methyl (S, E)-2-hydroxy-4-(4-methoxyphenyl)-2-((E)-styryl)but-3-enoate (ent-2h)

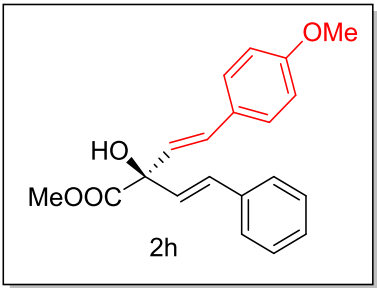

Colorless oil, $666 \%$ yield.

${ }^{1}$ H NMR $\left(300 \mathrm{MHz}, \mathrm{CDCl}_{3}\right): \delta 7.49-7.43(\mathrm{~m}, 2 \mathrm{H}), 7.43-7.26$ $(\mathrm{m}, 5 \mathrm{H}), 6.96-6.84(\mathrm{~m}, 4 \mathrm{H}), 6.50(\mathrm{~d}, J=15.8,1 \mathrm{H}), 6.36(\mathrm{~d}, J=$ $15.8,1 \mathrm{H}), 3.90(\mathrm{~s}, 3 \mathrm{H}), 3.87-3.83(\mathrm{~m}, 3 \mathrm{H}), 3.79(\mathrm{~s}, 1 \mathrm{H})$.

${ }^{13} \mathrm{C}$ NMR $\left(75 \mathrm{MHz}, \mathrm{CDCl}_{3}\right): \delta 174.46,159.45,136.19,129.94$, $129.59,128.87,128.55,128.51,127.96,127.87,126.72,126.36$,

$126.14,113.93,55.22,53.52$.

HRMS (ESI): $\mathrm{m} / \mathrm{z}$ Calcd. for $\left[\mathrm{C}_{20} \mathrm{H}_{20} \mathrm{NaO}_{4}, \mathrm{M}+\mathrm{Na}\right]^{+}: 347.1254$; Found: 347.1267.

Optical Rotation: 2h: $[\alpha]^{22}=-6.15\left(c=0.1, \mathrm{CH}_{2} \mathrm{Cl}_{2}\right)$. ent-2h: $[\alpha]^{22}=+16.50(\mathrm{c}=0.32$, $\mathrm{CH}_{2} \mathrm{Cl}_{2}$ ). The absolute configuration was assigned by analogy to that of ent-2i.

95.5:4.5 er. HPLC condition: Chiralcel OD-H column, $n$-hexane $/ i$-PrOH $=99: 1$, flow rate $=1$ $\mathrm{ml} / \mathrm{min}$, wavelength $=254 \mathrm{~nm}, \mathrm{t}_{\mathrm{R}}=22.322 \min$ for major isomer, $\mathrm{t}_{\mathrm{R}}=35.907 \mathrm{~min}$ for minor isomer.

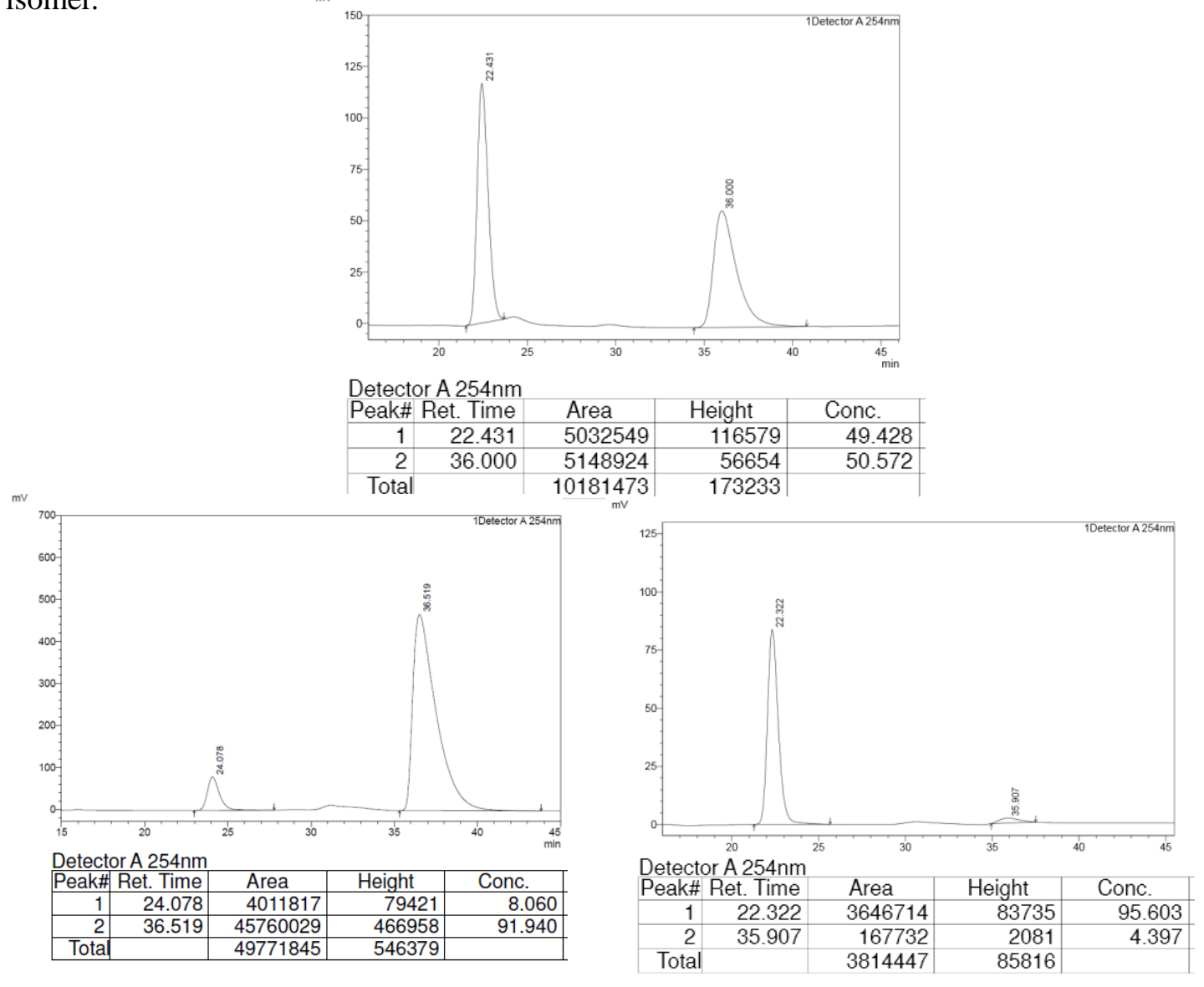




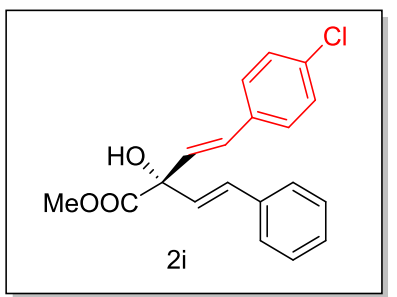

Pale yellow solid, $48 \%$ yield.

${ }^{1} \mathbf{H}$ NMR $\left(300 \mathrm{MHz}, \mathrm{CDCl}_{3}\right): \delta 7.53-7.42(\mathrm{~m}, 2 \mathrm{H}), 7.41-7.24$ $(\mathrm{m}, 7 \mathrm{H}), \delta 6.89(\mathrm{~d}, \mathrm{~J}=34.3 \mathrm{~Hz}, 1 \mathrm{H}) .6 .88(\mathrm{~d}, \mathrm{~J}=2.6 \mathrm{~Hz}, 1 \mathrm{H}), 6.48$ $(\mathrm{d}, \mathrm{J}=1.8 \mathrm{~Hz}, 1 \mathrm{H}), 6.44(\mathrm{~d}, \mathrm{~J}=1.7 \mathrm{~Hz}, 1 \mathrm{H}), 3.91(\mathrm{~s}, 3 \mathrm{H}), 3.82(\mathrm{~s}$, 1H)

${ }^{13}$ C NMR $\left(75 \mathrm{MHz}, \mathrm{CDCl}_{3}\right): \delta 174.15,136.02,134.65,130.17$, 128.96, 128.86, 128.69, 128.55, 128.13, 128.00, 127.95, 126.74, 53.65 .

HRMS (ESI): $\mathrm{m} / \mathrm{z}$ Calcd. for $\left[\mathrm{C}_{19} \mathrm{H}_{16} \mathrm{ClO}_{3}, \mathrm{M}-\mathrm{H}\right]^{-}: 327.0793$; Found: 327.0784 .

Optical Rotation: $[\alpha]^{22}=+11.50\left(\mathrm{c}=0.15, \mathrm{CH}_{2} \mathrm{Cl}_{2}\right)$. The absolute configuration was assigned by analogy to that of $\boldsymbol{e n t}-\mathbf{2 i}$.

96:4 er. HPLC condition: Chiralcel OD-H column, $n$-hexane $/ i-\mathrm{PrOH}=99: 1$, flow rate $=1$ $\mathrm{ml} / \mathrm{min}$, wavelength $=254 \mathrm{~nm}, \mathrm{t}_{\mathrm{R}}=25.732 \mathrm{~min}$ for major isomer, $\mathrm{t}_{\mathrm{R}}=33.422 \mathrm{~min}$ for minor isomer.
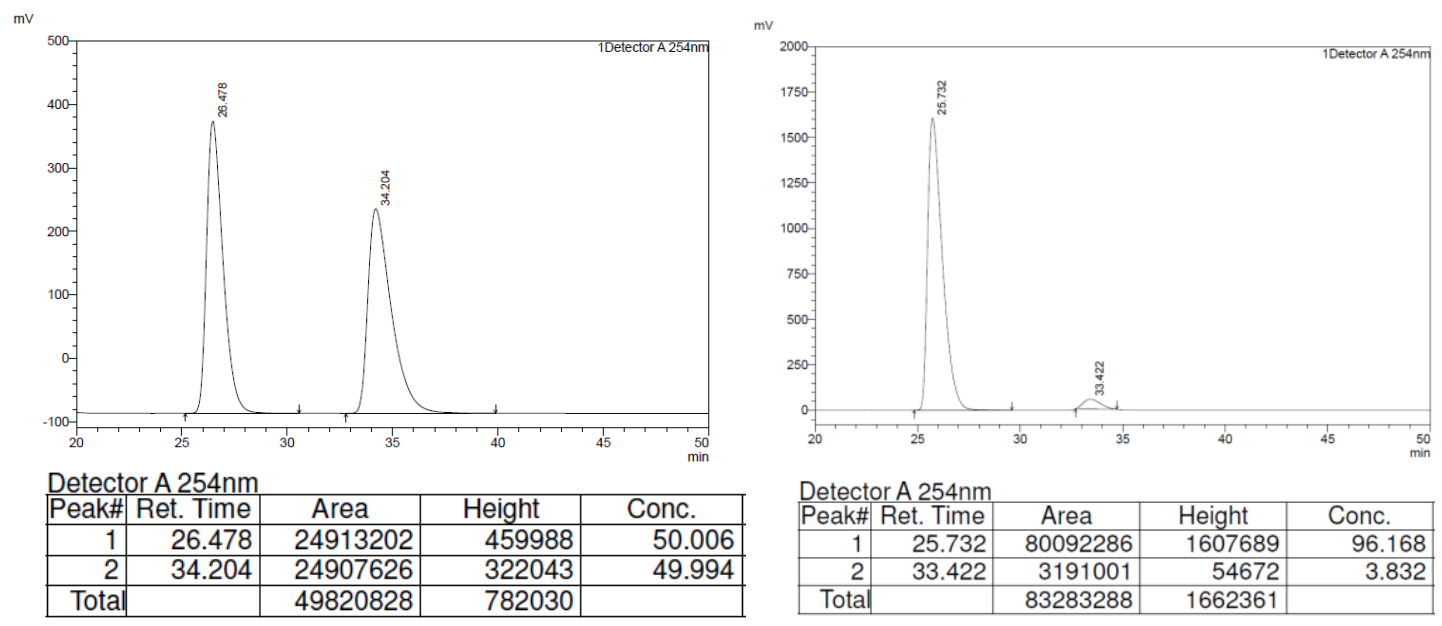

Methyl $(R, E)$-2-hydroxy-2-((E)-styryl)pent-3-enoate

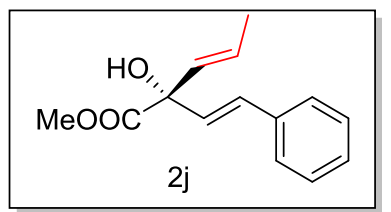

Colorless oil, $60 \%$ yield.

${ }^{1} \mathbf{H}$ NMR $\left(300 \mathrm{MHz}, \mathrm{CDCl}_{3}\right): \delta 7.49-7.39(\mathrm{~m}, 2 \mathrm{H}), 7.39-7.24$ $(\mathrm{m}, 3 \mathrm{H}), 6.87(\mathrm{~d}, J=15.9,1 \mathrm{H}), 6.42(\mathrm{~d}, J=15.9,1 \mathrm{H}), 6.07-5.93$ $(\mathrm{m}, 1 \mathrm{H}), 5.78(\mathrm{dq}, J=15.3,1.5,1 \mathrm{H}), 3.87(\mathrm{~s}, 3 \mathrm{H}), 3.83(\mathrm{~s}, 1 \mathrm{H})$, $1.79(\mathrm{dd}, J=6.5,1.6,3 \mathrm{H})$.

${ }^{13} \mathrm{C}$ NMR $\left(75 \mathrm{MHz}, \mathrm{CDCl}_{3}\right): \delta 174.64,136.26,130.34,129.61,128.76,128.48,128.37$, $127.77,126.94,126.67,126.35,53.33,17.58$.

HRMS (ESI): $\mathrm{m} / \mathrm{z}$ Calcd. for $\left[\mathrm{C}_{14} \mathrm{H}_{16} \mathrm{NaO}_{3}, \mathrm{M}+\mathrm{Na}\right]^{+}:$255.0992; Found: 255.0987.

Optical Rotation: $[\alpha]_{D}^{22}=-25.00\left(\mathrm{c}=0.24, \mathrm{CH}_{2} \mathrm{Cl}_{2}\right)$. The absolute configuration was assigned by analog to that of ent-2i.

95.5: 4.5 er. HPLC condition: Chiralpak AS-H column, $n$-hexane $/ i-\mathrm{PrOH}=99: 1$, flow rate $=$ 
$1 \mathrm{ml} / \mathrm{min}$, wavelength $=254 \mathrm{~nm}, t_{R}=16.817 \min$ for minor isomer, $t_{R}=20.036 \min$ for major isomer.
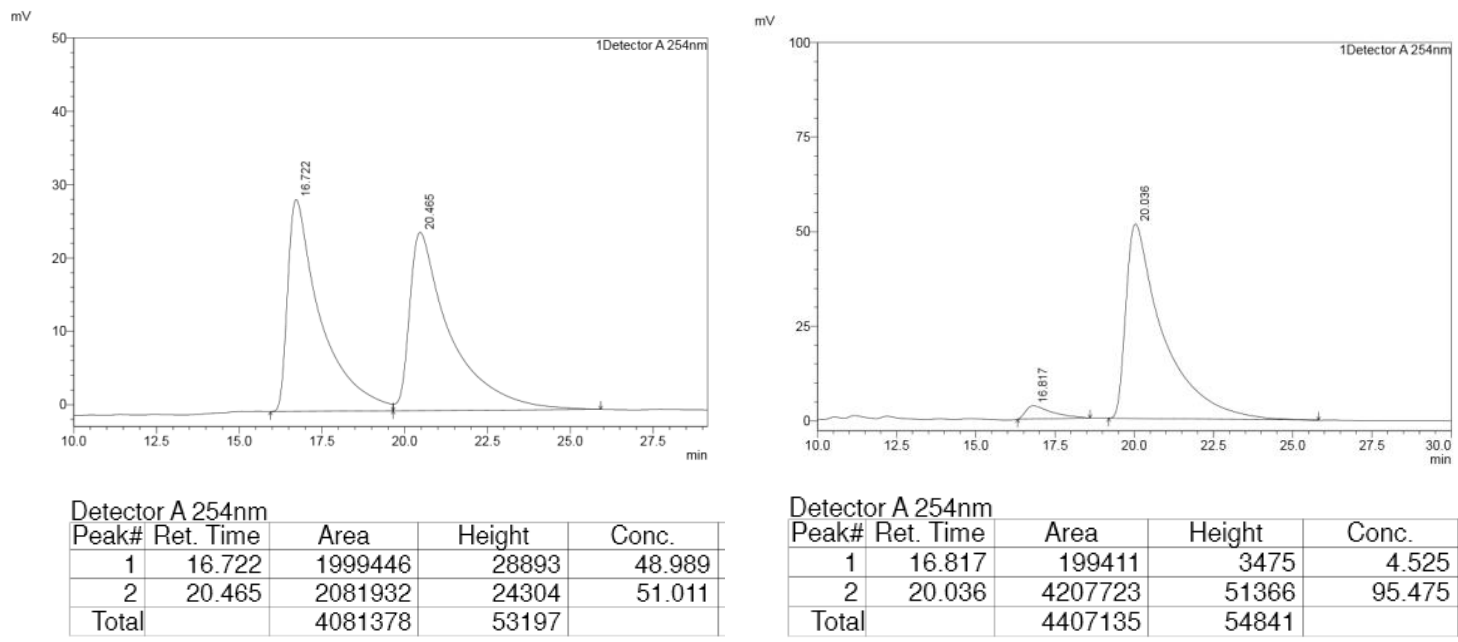

Methyl (R, E)-4-(3-chlorophenyl)-2-hydroxy-2-((E)-styryl)but-3-enoate

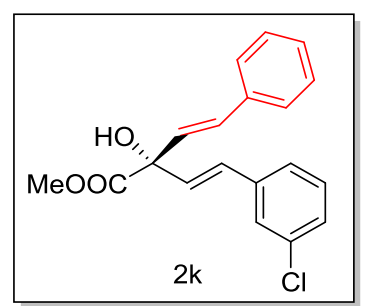

Colorless oil, $58 \%$ yield.

${ }^{1} \mathbf{H}$ NMR $\left(300 \mathrm{MHz}, \mathrm{CDCl}_{3}\right): 7.49-7.41(\mathrm{~m}, 3 \mathrm{H}), 7.36(\mathrm{~m}, 2 \mathrm{H})$, $7.32-7.21(\mathrm{~m}, 4 \mathrm{H}), 6.91(\mathrm{~d}, \mathrm{~J}=15.8 \mathrm{~Hz}, 1 \mathrm{H}), 6.86(\mathrm{~s}, 1 \mathrm{H}), 6.50(\mathrm{~d}$, $\mathrm{J}=15.8 \mathrm{~Hz}, 1 \mathrm{H}), 6.46(\mathrm{~d}, \mathrm{~J}=15.9 \mathrm{~Hz}, 2 \mathrm{H}) .3 .90(\mathrm{~s}, 3 \mathrm{H})$.

${ }^{13}$ C NMR $\left(125 \mathrm{MHz}, \mathrm{CDCl}_{3}\right): \delta=174.18,138.14,136.09,134.57$, $130.35,129.92,129.83,128.83,128.63,128.15,128.09,127.92$,

$126.83,126.57,125.22,53.75$.

HRMS (ESI): $\mathrm{m} / \mathrm{z}$ Calcd. for $\left[\mathrm{C}_{19} \mathrm{H}_{16} \mathrm{ClO}_{3}, \mathrm{M}-\mathrm{H}\right]=327.0793$; Found: 327.0784

Optical Rotation: $[\alpha]_{D}^{22}=-3.70\left(\mathrm{c}=0.2, \mathrm{CH}_{2} \mathrm{Cl}_{2}\right)$. The absolute configuration was assigned by analog to that of ent-2i.

96.5:3.5 er. HPLC condition: Chiralcel OD-H column, $n$-hexane $/ i-\operatorname{PrOH}=99: 1$, flow rate $=1$ $\mathrm{ml} / \mathrm{min}$, wavelength $=254 \mathrm{~nm}, \mathrm{t}_{\mathrm{R}}=32.055 \mathrm{~min}$ for minor isomer, $\mathrm{t}_{\mathrm{R}}=34.134 \mathrm{~min}$ for major isomer.
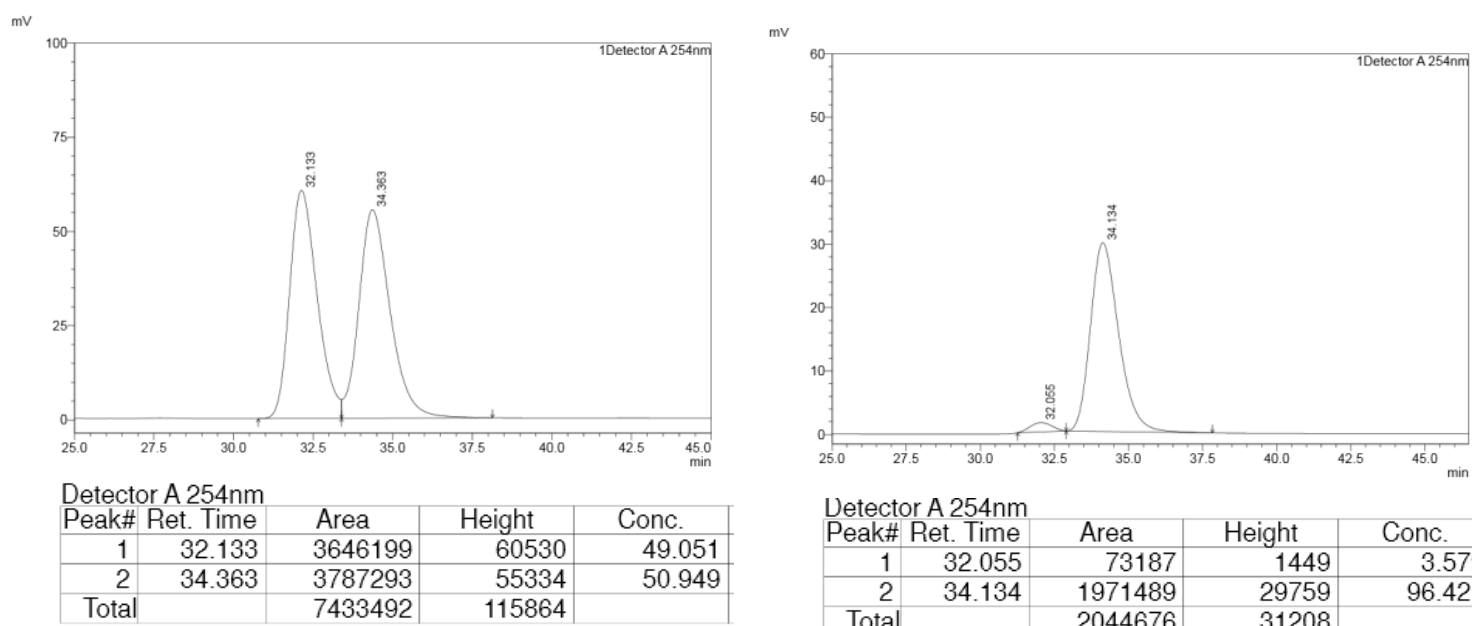

\begin{tabular}{|c|c|c|c|c|}
\hline & Area & Height & Conc. \\
\hline 1 & 32.055 & 73187 & 1449 & 3.579 \\
\hline 2 & 34.134 & 1971489 & 29759 & 96.421 \\
\hline Total & & 2044676 & 31208 & \\
\hline
\end{tabular}




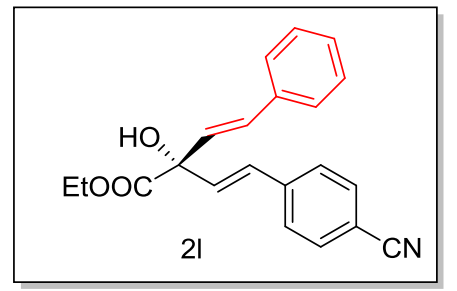

Colorless oil, $58 \%$ yield.

${ }^{1} \mathbf{H}$ NMR $\left(300 \mathrm{MHz}, \mathrm{CDCl}_{3}\right): \delta 7.74(\mathrm{~s}, 1 \mathrm{H}), 7.65(\mathrm{~d}, J=7.8$ $\mathrm{Hz}, 1 \mathrm{H}), 7.56$ (dd, $J=5.2,3.8 \mathrm{~Hz}, 1 \mathrm{H}), 7.53-7.43(\mathrm{~m}, 3 \mathrm{H})$, $7.41-7.29(\mathrm{~m}, 3 \mathrm{H}), 6.96(\mathrm{~s}, 1 \mathrm{H}), 6.91(\mathrm{~s}, 1 \mathrm{H}), 6.58(\mathrm{~d}, J=$ $15.8 \mathrm{~Hz}, 1 \mathrm{H}), 6.47(\mathrm{~d}, J=15.8 \mathrm{~Hz}, 1 \mathrm{H}), 4.38(\mathrm{q}, J=7.1 \mathrm{~Hz}$, $2 \mathrm{H}), 3.90(\mathrm{~s}, 1 \mathrm{H}), 1.41(\mathrm{t}, J=7.1 \mathrm{~Hz}, 3 \mathrm{H})$.

${ }^{13} \mathrm{C}$ NMR $\left(75 \mathrm{MHz}, \mathrm{CDCl}_{3}\right): \delta 173.38,137.54,135.96,131.23,131.05,130.27,130.02$, 129.36, 128.56, 128.05, 127.93, 127.70, 126.74, 118.60, 112.77, 63.17, 14.12 .

HRMS (ESI): $\mathrm{m} / \mathrm{z}$ Calcd. For $\left[\mathrm{C}_{21} \mathrm{H}_{19} \mathrm{NNaO}_{3}, \mathrm{M}+\mathrm{Na}\right]$ : 356.1257; Found: 356.1262.

Optical Rotation: $[\alpha]_{D}^{22}=-15.50\left(\mathrm{c}=0.30, \mathrm{CH}_{2} \mathrm{Cl}_{2}\right)$. The absolute configuration was assigned by analog to that of $\boldsymbol{e n t}-\mathbf{2 i}$.

94:6 er. HPLC condition: Chiralcel OD-H column, $n$-hexane $/ i-\mathrm{PrOH}=92: 8$, flow rate $=1$ $\mathrm{ml} / \mathrm{min}$, wavelength $=254 \mathrm{~nm}, \mathrm{t}_{\mathrm{R}}=20.030 \mathrm{~min}$ for minor isomer, $\mathrm{t}_{\mathrm{R}}=23.779$ min for major isomer.
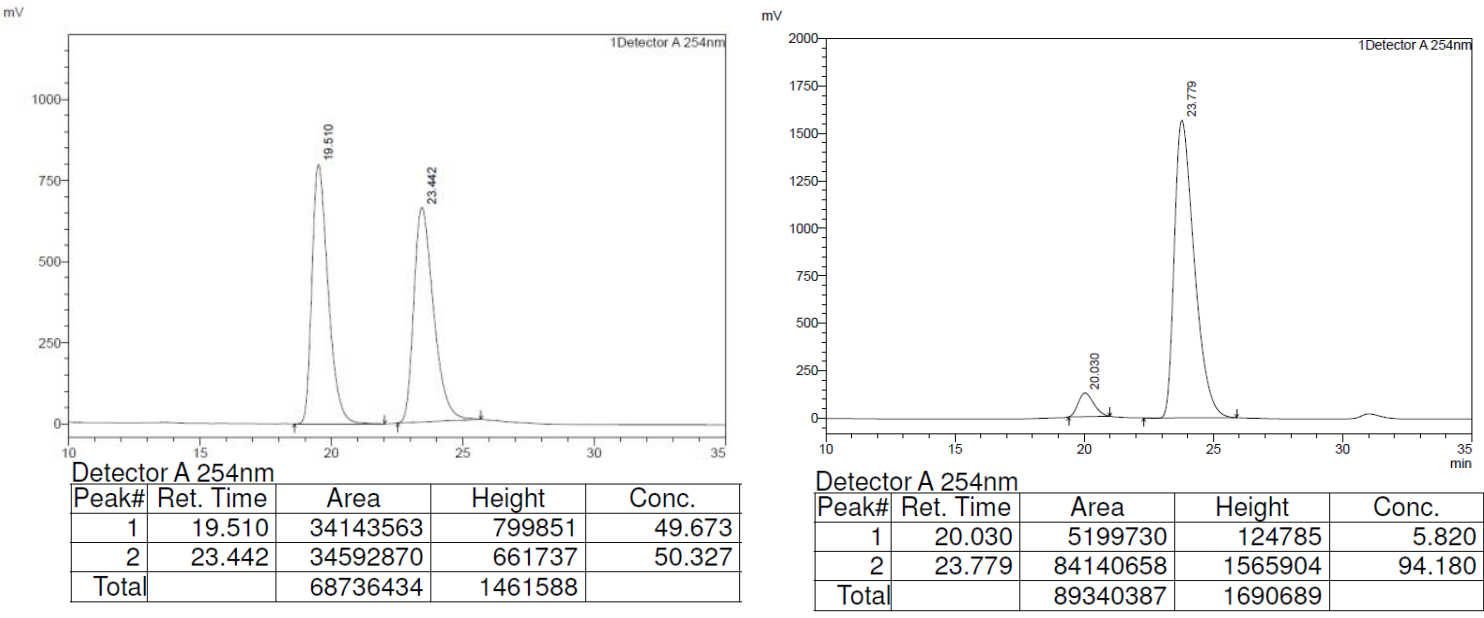

Methyl $(R, E)$-2-hydroxy-2-((E)-4-methylstyryl)-4-phenylbut-3-enoate

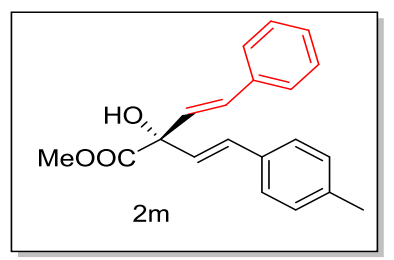

Colorless oil, $55 \%$ yield.

${ }^{1} \mathbf{H}$ NMR $\left(500 \mathrm{MHz}, \mathrm{CDCl}_{3}\right): \delta 7.45(\mathrm{~d}, J=7.5,2 \mathrm{H}), 7.38-7.33$ (m, 4H), $7.32-7.24(\mathrm{~m}, 1 \mathrm{H}), 7.16(\mathrm{~d}, J=7.9,2 \mathrm{H}), 6.91(\mathrm{~s}, 1 \mathrm{H})$, $6.90(\mathrm{~d}, \mathrm{~J}=32.4 \mathrm{~Hz}, 1 \mathrm{H}), 6.49(\mathrm{~d}, \mathrm{~J}=15.8 \mathrm{~Hz}, 1 \mathrm{H}), 6.43(\mathrm{~d}, \mathrm{~J}=$ $15.8 \mathrm{~Hz}, 1 \mathrm{H}), 3.89(\mathrm{~d}, J=3.8,3 \mathrm{H}), 3.78(\mathrm{~s}, 1 \mathrm{H}), 2.37$ (s, 3H).

${ }^{13}$ C NMR $\left(125 \mathrm{MHz}, \mathrm{CDCl}_{3}\right): \delta=174.49,137.91,136.28,133.44$, $130.11,129.32,128.61,127.98,127.45,126.82,126.73,53.61,21.24$.

HRMS (ESI): $\mathrm{m} / \mathrm{z}$ Calcd. for $\left[\mathrm{C}_{20} \mathrm{H}_{20} \mathrm{NaO}_{3}, \mathrm{M}+\mathrm{Na}\right]^{+}: 331.1305$; Found: 331.1312 .

Optical Rotation: $\left[[\alpha]^{22}=-12.60\left(\mathrm{c}=0.16, \mathrm{CH}_{2} \mathrm{Cl}_{2}\right)\right.$. The absolute configuration was assigned by analog to that of $\boldsymbol{e n t} \mathbf{- 2 i}$. 
96.5:3.5 er. HPLC condition: Chiralcel OD-H column, $n$-hexane $/ i$-PrOH $=99: 1$, flow rate $=1$ $\mathrm{ml} / \mathrm{min}$, wavelength $=254 \mathrm{~nm}, t_{R}=23.341 \mathrm{~min}$ for minor isomer, $t_{R}=25.713 \mathrm{~min}$ for major isomer.
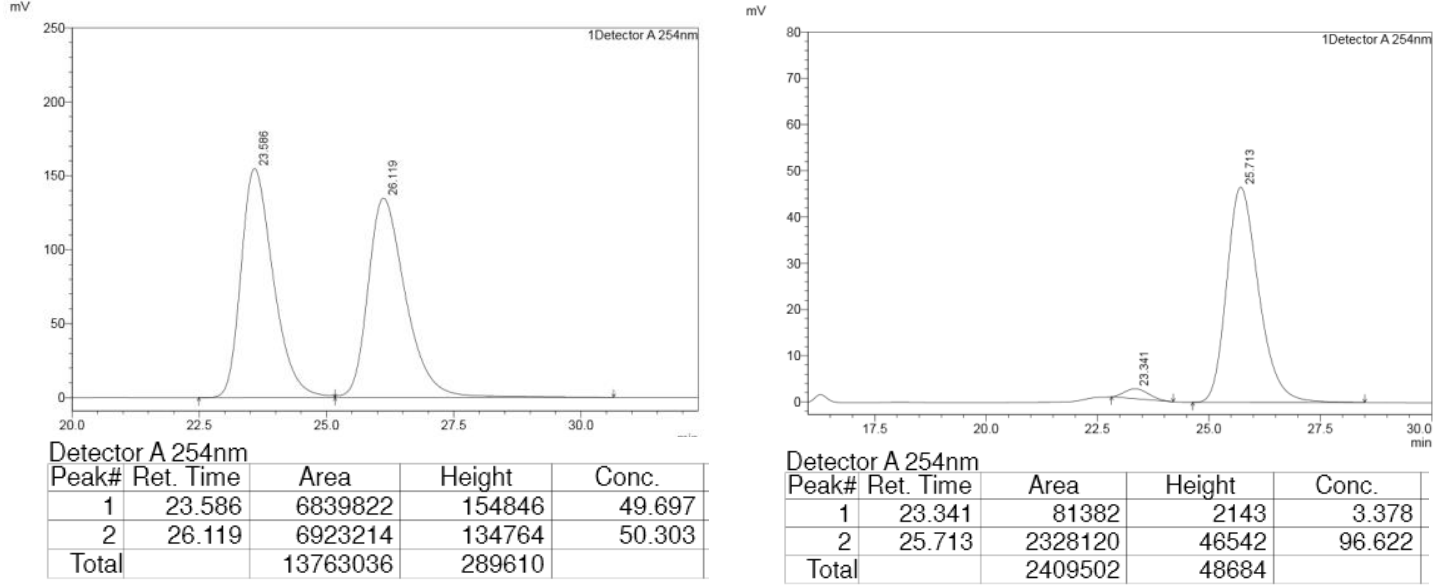

Methyl $(R, E)$-2-hydroxy-4-phenyl-2-((E)-4-(trifluoromethyl)styryl)but-3-enoate

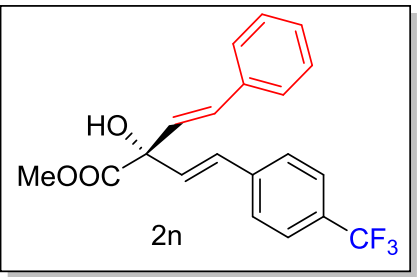

Colorless oil, $55 \%$ yield.

${ }^{1} \mathbf{H}$ NMR $\left(500 \mathrm{MHz}, \mathrm{CDCl}_{3}\right): \delta 7.60(\mathrm{~d}, J=8.2,2 \mathrm{H}), 7.54(\mathrm{~d}, J$ $=8.2,2 \mathrm{H}), 7.45(\mathrm{~d}, J=7.7,2 \mathrm{H}), 7.36(\mathrm{t}, J=7.6,2 \mathrm{H}), 7.30(\mathrm{dd}$, $J=7.3,1 \mathrm{H}), 6.93(\mathrm{~d}, \mathrm{~J}=5 \mathrm{~Hz}, 1 \mathrm{H}), 6.92(\mathrm{~d}, \mathrm{~J}=5 \mathrm{~Hz}, 1 \mathrm{H})$, $6.58(\mathrm{~d}, J=15.8,1 \mathrm{H}), 6.47(\mathrm{~d}, J=15.8,1 \mathrm{H}), 3.91(\mathrm{~s}, 3 \mathrm{H})$.

${ }^{13} \mathbf{C}$ NMR $\left(125 \mathrm{MHz}, \mathrm{CDCl}_{3}\right): \delta 174.06,139.77,136.06$,

131.07, 130.46, 128.81, 128.64, 128.14, 128.05, 127.44(q, $\left.J_{F C}=237.4 \mathrm{~Hz}\right), 126.99,126.83$, $125.55\left(\mathrm{q}, J_{F C}=15 \mathrm{~Hz}\right), 53.76$.

${ }^{19}$ F NMR $\left(282 \mathrm{MHz}, \mathrm{CDCl}_{3}\right): \delta-62.52$.

HRMS (ESI): $\mathrm{m} / \mathrm{z}$ Calcd. for $\left[\mathrm{C}_{20} \mathrm{H}_{16} \mathrm{~F}_{3} \mathrm{O}_{3}, \mathrm{M}-\mathrm{H}\right]^{-}: 361.1057$; Found: 361.1063 .

Optical Rotation: $[\alpha]^{22}=-24.66\left(c=0.20, \mathrm{CH}_{2} \mathrm{Cl}_{2}\right)$. The absolute configuration was assigned by analog to that of $\boldsymbol{e n t} \mathbf{- 2 i}$.

95.5:4.5 er. HPLC condition: Chiralcel OD-H column, $n$-hexane $/ i$-PrOH $=99: 1$, flow rate $=1$ $\mathrm{ml} / \mathrm{min}$, wavelength $=254 \mathrm{~nm}, \mathrm{t}_{\mathrm{R}}=25.121 \mathrm{~min}$ for minor isomer, $\mathrm{t}_{\mathrm{R}}=29.647 \mathrm{~min}$ for major isomer.
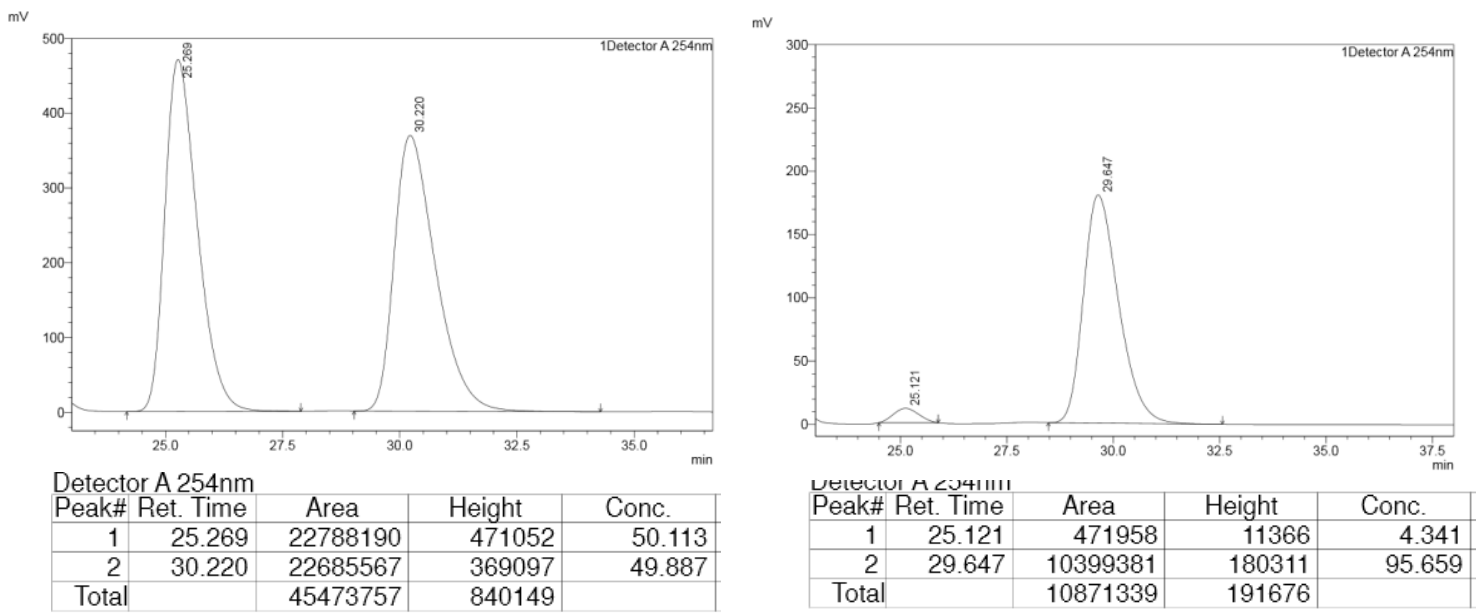
Methyl (R, E)-4-(4-bromophenyl)-2-hydroxy-2-((E)-styryl)but-3-enoate

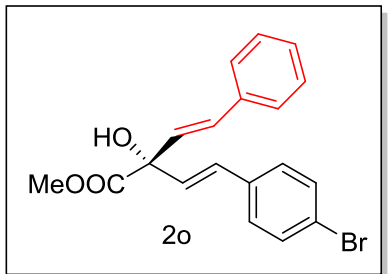

$121.83,53.73$.

colorless oil, $60 \%$ yield.

${ }^{1}$ H NMR $\left(500 \mathrm{MHz}, \mathrm{CDCl}_{3}\right): \delta 7.45(\mathrm{dt}, J=23.6,8.8,4 \mathrm{H}), 7.38$ $-7.23(\mathrm{~m}, 5 \mathrm{H}), 6.92(\mathrm{~d}, \mathrm{~J}=15.9 \mathrm{~Hz}, 1 \mathrm{H}), 6.86(\mathrm{~d}, \mathrm{~J}=15.9 \mathrm{~Hz}$, $1 \mathrm{H}), 6.49$ (s, 1H), $6.46(\mathrm{~d}, \mathrm{~J}=7.0 \mathrm{~Hz}, 1 \mathrm{H}) .3 .90$ (s, 3H).

${ }^{13}$ C NMR $\left(125 \mathrm{MHz}, \mathrm{CDCl}_{3}\right): \delta 174.19,136.11,135.20,131.73$, $130.29,129.20,129.02,128.63,128.35,128.20,128.09,126.83$,

HRMS (ESI): $\mathrm{m} / \mathrm{z}$ Calcd. for $\left[\mathrm{C}_{19} \mathrm{H}_{16} \mathrm{BrO}_{3}, \mathrm{M}-\mathrm{H}\right]^{-}: 371.0288$; Found: 371.0290.

Optical Rotation: $\alpha]_{D}^{22}=-4.50\left(\mathrm{c}=0.20, \mathrm{CH}_{2} \mathrm{Cl}_{2}\right)$. The absolute configuration was assigned by analog to that of $\boldsymbol{e n t}-\mathbf{2 i}$.

95.5:4.5 er. HPLC condition: Chiralcel OD-H column, $n$-hexane $/ i-\operatorname{PrOH}=99: 1$, flow rate $=1$ $\mathrm{ml} / \mathrm{min}$, wavelength $=254 \mathrm{~nm}, \mathrm{t}_{\mathrm{R}}=29.640 \mathrm{~min}$ for minor isomer, $\mathrm{t}_{\mathrm{R}}=38.409 \mathrm{~min}$ for major isomer.
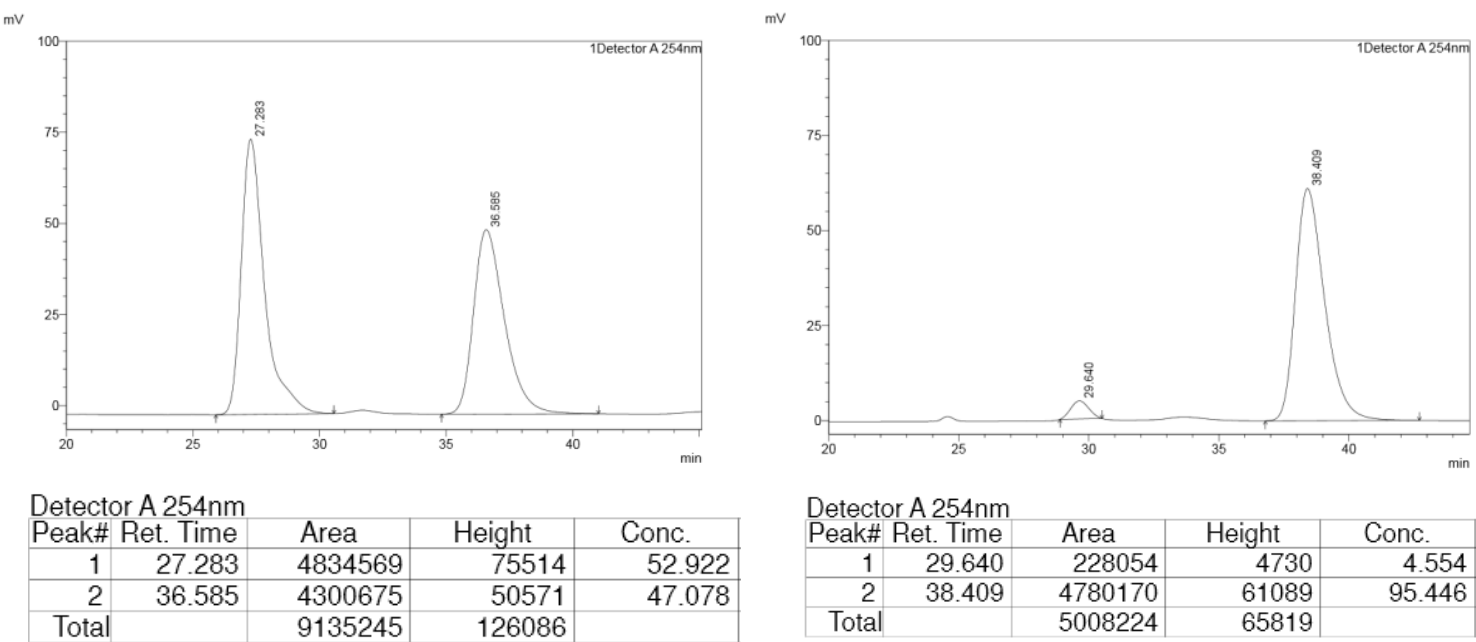

Methyl $(R, E)-4-(4-f l u o r o p h e n y l)-2-h y d r o x y-2-((E)$-styryl)but-3-enoate

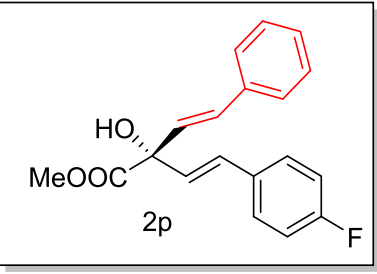

$115.31,53.61$.
Colorless oil, $62 \%$ yield.

${ }^{1} \mathbf{H}$ NMR $\left(300 \mathrm{MHz}, \mathrm{CDCl}_{3}\right): \delta 7.52-7.26(\mathrm{~m}, 8 \mathrm{H}), 7.12-7.00$ (m, 2H), $6.91(\mathrm{dd}, J=15.8,9.6,2 \mathrm{H}), 6.44(\mathrm{dd}, J=20.8,15.8$, $2 \mathrm{H}), 3.91(\mathrm{~s}, 3 \mathrm{H})$.

${ }^{13} \mathbf{C}$ NMR $\left(75 \mathrm{MHz}, \mathrm{CDCl}_{3}\right): \delta 174.25,136.06,132.31,130.10$, $128.93,128.53,128.35,128.25,128.07,127.97,126.73,115.60$, 
${ }^{19}$ F NMR $\left(282 \mathrm{MHz}, \mathrm{CDCl}_{3}\right): \delta-113.61--113.69(\mathrm{~m}, 1 \mathrm{~F})$.

HRMS (ESI): $\mathrm{m} / \mathrm{z}$ Calcd. for $\left[\mathrm{C}_{19} \mathrm{H}_{16} \mathrm{FO}_{3}, \mathrm{M}-\mathrm{H}\right]^{-}:$: 311.1089; Found: 311.1086.

Optical Rotation: $[\alpha]_{D}^{22}=-7.30\left(\mathrm{c}=0.25 \mathrm{CH}_{2} \mathrm{Cl}_{2}\right)$. The absolute configuration was assigned by analog to that of ent-2i.

97:3 er. HPLC condition: Chiralcel OD-H column, $n$-hexane $/ i-\mathrm{PrOH}=99: 1$, flow rate $=1$ $\mathrm{ml} / \mathrm{min}$, wavelength $=254 \mathrm{~nm}, \mathrm{t}_{\mathrm{R}}=25.508 \mathrm{~min}$ for minor isomer, $\mathrm{t}_{\mathrm{R}}=32.382 \mathrm{~min}$ for major isomer.
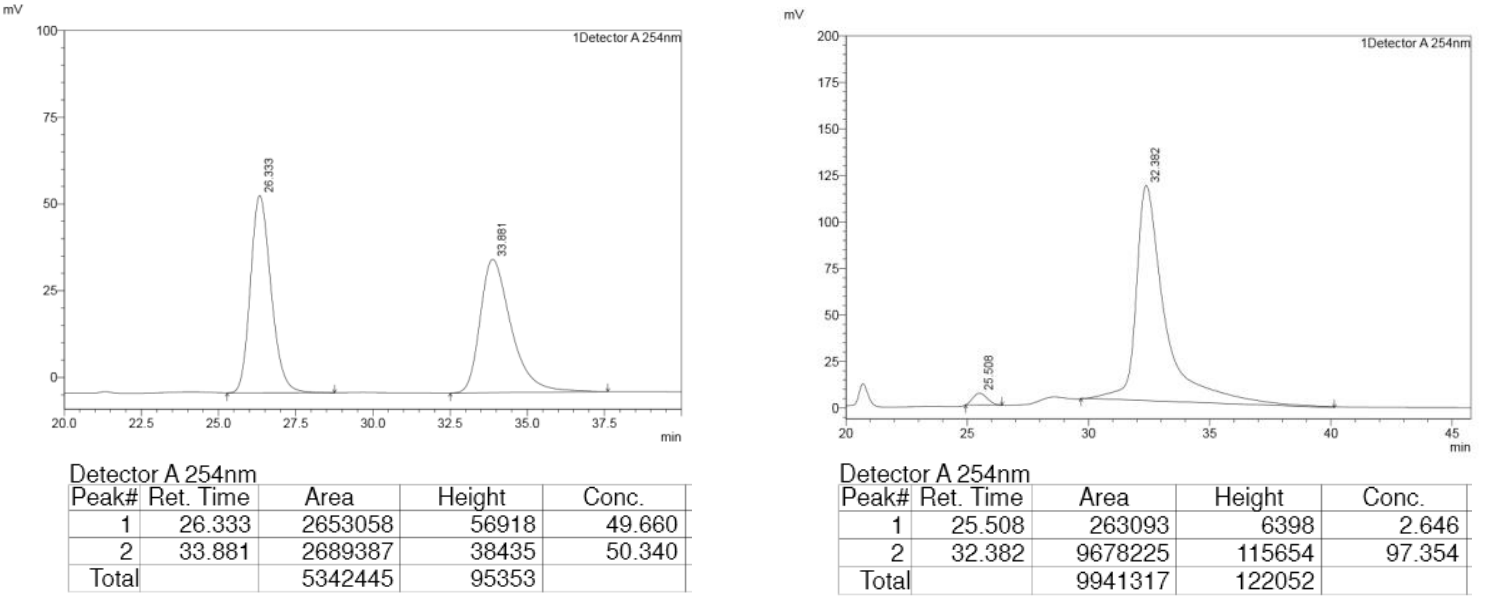

Methyl $(R, E)-4-(4-c h l o r o p h e n y l)-2-h y d r o x y-2-((E)$-styryl)but-3-enoate

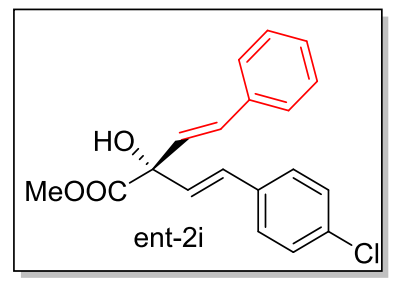

Pale yellow solid, $75 \%$ yield.

${ }^{1} \mathbf{H}$ NMR $\left(300 \mathrm{MHz}, \mathrm{CDCl}_{3}\right): \delta 7.46-7.44(\mathrm{~m}, 2 \mathrm{H}), 7.41-$ $7.24(\mathrm{~m}, 7 \mathrm{H}), \delta 6.89(\mathrm{~d}, \mathrm{~J}=34.3 \mathrm{~Hz}, 1 \mathrm{H}) .6 .88(\mathrm{~d}, \mathrm{~J}=2.6 \mathrm{~Hz}$, $1 \mathrm{H}), 6.48(\mathrm{~d}, \mathrm{~J}=1.8 \mathrm{~Hz}, 1 \mathrm{H}), 6.45(\mathrm{~d}, \mathrm{~J}=1.7 \mathrm{~Hz}, 1 \mathrm{H}), 3.91$ $(\mathrm{s}, 3 \mathrm{H}), 3.82(\mathrm{~s}, 1 \mathrm{H})$.

${ }^{13} \mathrm{C}$ NMR $\left(75 \mathrm{MHz}, \mathrm{CDCl}_{3}\right): \delta 174.15,145.12,136.02,134.65$, 133.57, 130.17, 128.95, 128.86, 128.69, 128.54, 128.13, 127.99, 127.95, 126.73, 53.65.

HRMS (ESI): m/z Calcd. For $\left[\mathrm{C}_{19} \mathrm{H}_{16} \mathrm{ClO}_{3}, \mathrm{M}-\mathrm{H}\right]=327.0793$; Found: 327.0791.

Optical Rotation: $[\alpha]_{D}^{22}=-7.67\left(\mathrm{c}=0.12, \mathrm{CH}_{2} \mathrm{Cl}_{2}\right)$. The absolute configuration was assigned by X-ray crystallography.

96.5:3.5 er. HPLC condition: Chiralcel OD-H column, $n$-hexane $/ i$-PrOH $=99: 1$, flow rate $=1$ $\mathrm{ml} / \mathrm{min}$, wavelength $=254 \mathrm{~nm}, \mathrm{t}_{\mathrm{R}}=27.238 \mathrm{~min}$ for minor isomer, $\mathrm{t}_{\mathrm{R}}=34.185 \mathrm{~min}$ for major isomer.
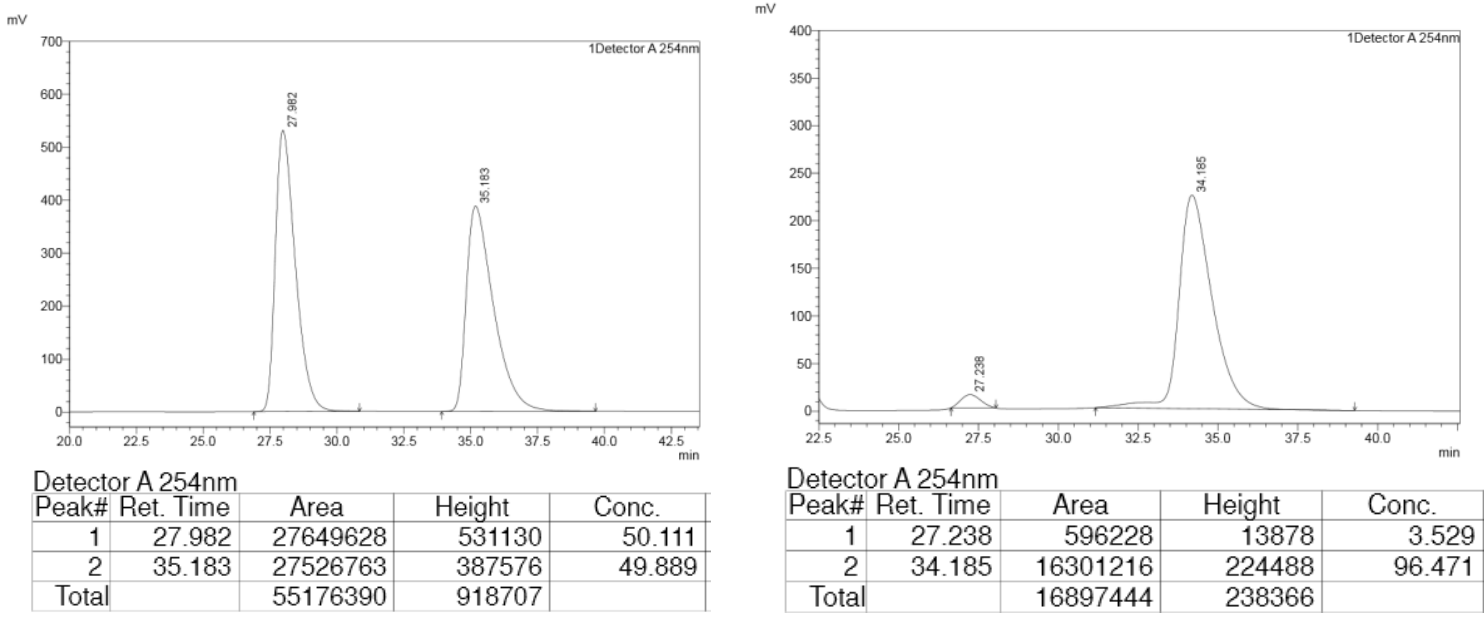


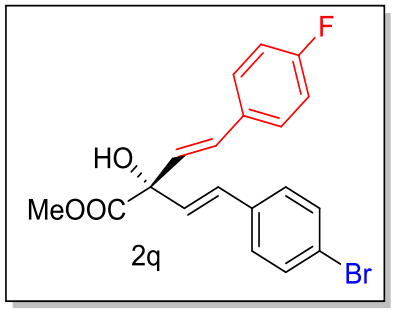

Pale yellow solid, $60 \%$ yield.

${ }^{1} \mathbf{H}$ NMR $\left(500 \mathrm{MHz}, \mathrm{CDCl}_{3}\right): \delta 7.48(\mathrm{~s}, 1 \mathrm{H}), 7.46(\mathrm{~s}, 1 \mathrm{H}), 7.40$ $(\mathrm{m}, 2 \mathrm{H}), 7.31(\mathrm{~s}, 1 \mathrm{H}), 7.29(\mathrm{~d}, \mathrm{~J}=2.6 \mathrm{~Hz}, 1 \mathrm{H}), 6.88(\mathrm{~d}, \mathrm{~J}=8.9 \mathrm{~Hz}$, $1 \mathrm{H}), 6.84(\mathrm{~d}, \mathrm{~J}=8.9 \mathrm{~Hz}, 1 \mathrm{H}), 6.46(\mathrm{~d}, \mathrm{~J}=15.8 \mathrm{~Hz}, 1 \mathrm{H}), 6.37(\mathrm{~d}, \mathrm{~J}$ $=15.8 \mathrm{~Hz}, 1 \mathrm{H}) .3 .90(\mathrm{~s}, 3 \mathrm{H})$.

${ }^{13}$ C NMR (125 MHz, $\left.\mathrm{CDCl}_{3}\right): \delta=174.11,163.59,161.62,135.15$, $132.31,131.73,129.13,129.04,128.42,128.35,128.33,127.93$,

${ }^{19}$ F NMR $\left(282 \mathrm{MHz}, \mathrm{CDCl}_{3}\right): \delta-113.33--113.78(\mathrm{~m}, 1 \mathrm{~F})$.

HRMS (ESI): $\mathrm{m} / \mathrm{z}$ Calcd. for $\left[\mathrm{C}_{19} \mathrm{H}_{15} \mathrm{BrFO}_{3}, \mathrm{M}-\mathrm{H}\right]: 389.0194$; Found: 389.0190 .

Optical Rotation: $[\alpha]^{22}=-14.83\left(\mathrm{c}=0.30, \mathrm{CH}_{2} \mathrm{Cl}_{2}\right)$. The absolute configuration was assigned by analog to that of $\boldsymbol{e n t}$-2i.

97.5:2.5 er. HPLC condition: Chiralcel OD-H column, $n$-hexane $/ i$ - $\operatorname{PrOH}=99: 1$, flow rate $=1$ $\mathrm{ml} / \mathrm{min}$, wavelength $=254 \mathrm{~nm}, \mathrm{t}_{\mathrm{R}}=36.688 \mathrm{~min}$ for minor isomer, $\mathrm{t}_{\mathrm{R}}=39.461 \mathrm{~min}$ for major isomer.
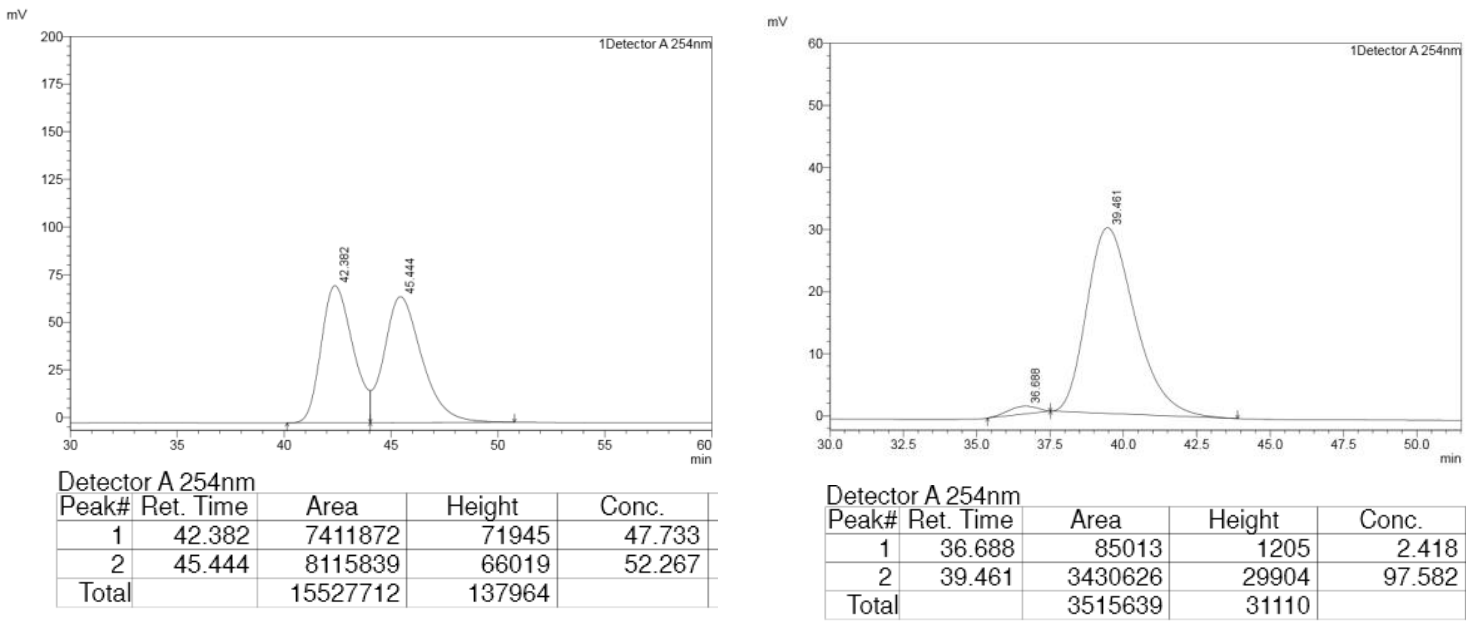

\section{Methyl (R, E)-4-(benzofuran-2-yl)-2-hydroxy-2-((E)-styryl)but-3-enoate}

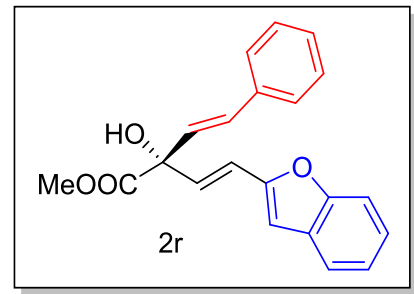

Colorless oil, $60 \%$ yield.

${ }^{1} \mathbf{H}$ NMR $\left(500 \mathrm{MHz}, \mathrm{CDCl}_{3}\right): \delta 7.55(\mathrm{~d}, J=7.6,1 \mathrm{H}), 7.52-$ $7.43(\mathrm{~m}, 3 \mathrm{H}), 7.41-7.27(\mathrm{~m}, 4 \mathrm{H}), 7.22(\mathrm{t}, J=7.5,1 \mathrm{H}), 6.93(\mathrm{~d}$, $\mathrm{J}=15.8 \mathrm{~Hz}, 1 \mathrm{H}), 6.87(\mathrm{~s}, 1 \mathrm{H}), 6.74(\mathrm{~d}, \mathrm{~J}=15.6 \mathrm{~Hz}, 1 \mathrm{H}), 6.48$ (d, J = $15.8 \mathrm{~Hz}, 1 \mathrm{H}) .3 .92(\mathrm{~s}, 3 \mathrm{H}), 3.84(\mathrm{~s}, 1 \mathrm{H})$.

${ }^{13} \mathbf{C}$ NMR $\left(125 \mathrm{MHz}, \mathrm{CDCl}_{3}\right): \delta=174.14,153.86,136.13$, $130.37,130.06,128.62,128.07,126.84,124.82,122.88,121.07$,

$118.55,110.92,106.00,53.82$.

HRMS (ESI): $\mathrm{m} / \mathrm{z}$ Calcd. for $\left[\mathrm{C}_{21} \mathrm{H}_{18} \mathrm{NaO}_{4}, \mathrm{M}+\mathrm{Na}\right]^{+}: 357.1097$; Found: 357.1111.

Optical Rotation: $[\alpha]^{22}=-13.45\left(\mathrm{c}=0.30, \mathrm{CH}_{2} \mathrm{Cl}_{2}\right)$. The absolute configuration was assigned 
by analog to that of ent-2i.

96:4 er. HPLC condition: Chiralpark OD-H column, $n$-hexane $/ i-\operatorname{PrOH}=99: 1$, flow rate $=1$ $\mathrm{ml} / \mathrm{min}$, wavelength $=254 \mathrm{~nm}, \mathrm{t}_{\mathrm{R}}=19.580 \min$ for minor isomer, $\mathrm{t}_{\mathrm{R}}=21.822 \mathrm{~min}$ for major isomer.
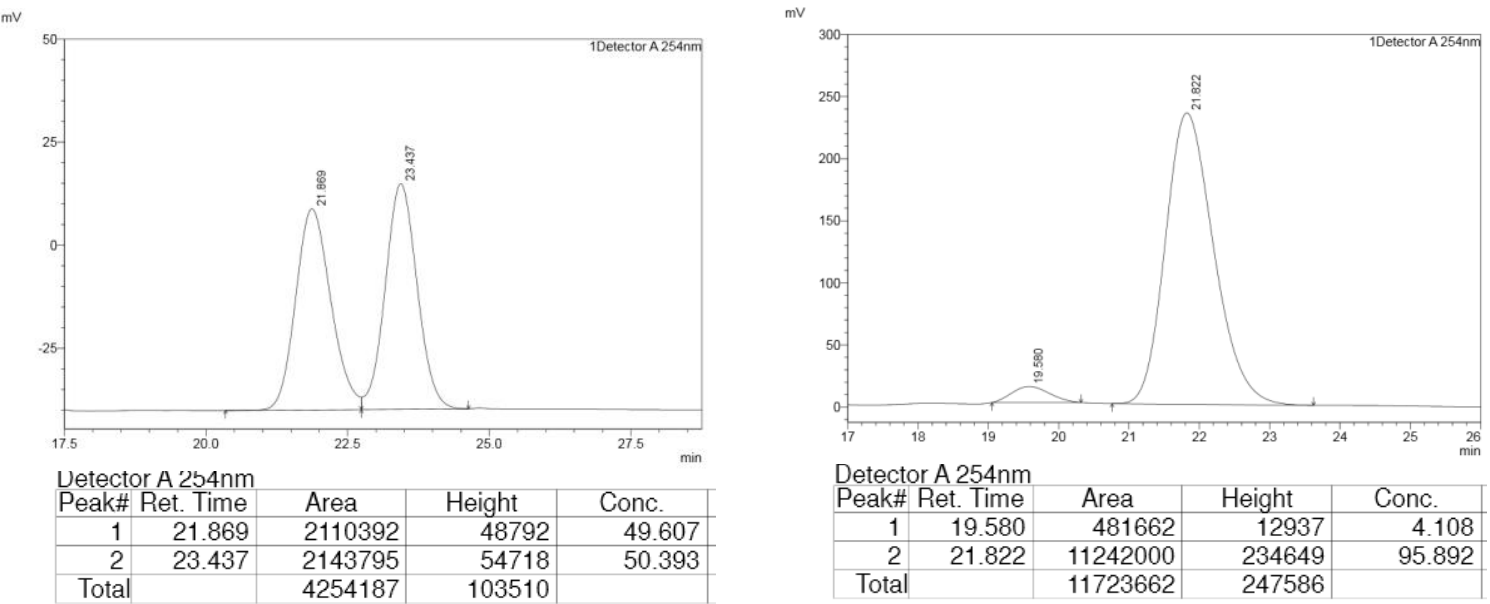

Ethyl $(S, 3 E, 5 E)$-2-hydroxy-6-phenyl-2-((E)-styryl)hexa-3,5-dienoate

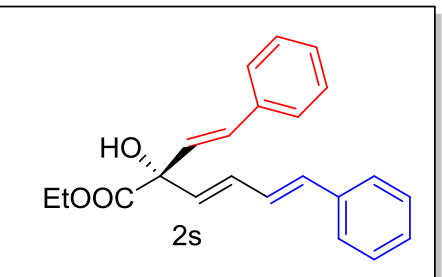

Colorless oil, $40 \%$ yield.

${ }^{1} \mathbf{H}$ NMR $\left(500 \mathrm{MHz}, \mathrm{CDCl}_{3}\right): \delta 7.43(\mathrm{~m}, 4 \mathrm{H}), 7.35(\mathrm{~m}, \mathrm{H})$, $7.27(\mathrm{~m}, 2 \mathrm{H}), 6.90(\mathrm{~d}, \mathrm{~J}=15.8 \mathrm{~Hz}, 1 \mathrm{H}), 6.84(\mathrm{dd}, \mathrm{J}=15.6$, $10.6 \mathrm{~Hz}, 1 \mathrm{H}), 6.71(\mathrm{dd}, \mathrm{J}=15.0,10.7 \mathrm{~Hz}, 1 \mathrm{H}), 6.64(\mathrm{~d}, \mathrm{~J}=$ $15.6 \mathrm{~Hz}, 1 \mathrm{H}), 6.43(\mathrm{~d}, \mathrm{~J}=15.8 \mathrm{~Hz}, 1 \mathrm{H}), 6.09(\mathrm{~d}, \mathrm{~J}=15.0 \mathrm{~Hz}$, $1 \mathrm{H}) .4 .39-4.28(\mathrm{~m}, 2 \mathrm{H}), 3.76(\mathrm{~s}, 1 \mathrm{H}), 1.37$ (t, $J=7.2,3 \mathrm{H})$.

${ }^{13} \mathbf{C}$ NMR (125 MHz, $\left.\mathrm{CDCl}_{3}\right): \delta=173.86,137.06,133.95,132.35,130.55,129.99,128.63$, 128.59, 127.93, 127.75, 127.71, 126.80, 126.48, 62.94, 14.18.

HRMS (ESI): $\mathrm{m} / \mathrm{z}$ Calcd. for $\left[\mathrm{C}_{21} \mathrm{H}_{20} \mathrm{NaO}_{3}, \mathrm{M}+\mathrm{Na}\right]^{+}: 343.1305$; Found: 343.1314 .

Optical Rotation: $[\alpha]^{22}{ }_{D}=-5.6\left(c=0.5, \mathrm{CH}_{2} \mathrm{Cl}_{2}\right)$. The absolute configuration was assigned by analog to that of ent-2i.

92:8 er. HPLC condition: Chiralpak AS-H column, $n$-hexane $/ i$-PrOH $=99: 1$, flow rate $=1$ $\mathrm{ml} / \mathrm{min}$, wavelength $=254 \mathrm{~nm}, \mathrm{t}_{\mathrm{R}}=25.005$ min for major isomer, $\mathrm{t}_{\mathrm{R}}=31.829 \min$ for minor isomer.
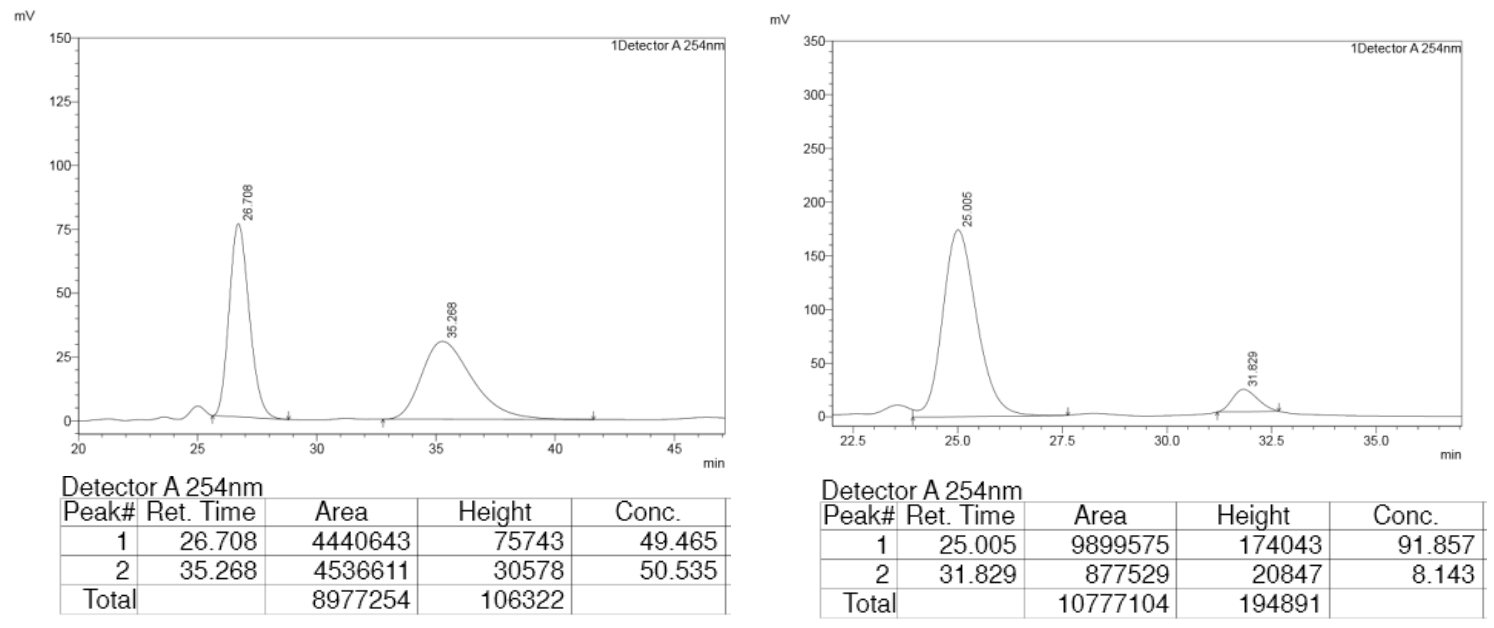


\section{Analytical data of vinylation products 7}

\section{(R, E)-3-hydroxy-3-styrylindolin-2-one}

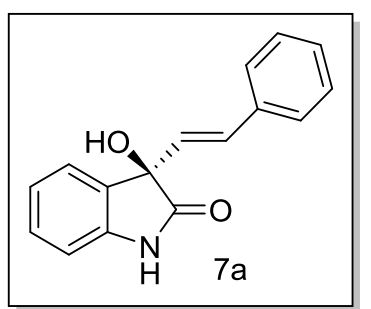

Known Compound ${ }^{3}$, white solid, $80 \%$ yield.

${ }^{1}$ H NMR (300 MHz, DMSO): $\delta=10.38$ (s, 1H), $7.47-7.36(\mathrm{~m}, 2 \mathrm{H})$, $7.36-7.19(\mathrm{~m}, 5 \mathrm{H}), 7.06-6.96(\mathrm{~m}, 1 \mathrm{H}), 6.87(\mathrm{~d}, J=7.6,1 \mathrm{H}), 6.56(\mathrm{~d}$, $J=16.0,1 \mathrm{H}), 6.39$ (s, 1H), 6.35 (d, $J=16.0,1 \mathrm{H})$

${ }^{13}$ C NMR (75 MHz, DMSO): $\delta$ 178.29, 142.07, 136.43, 132.20, $129.88,129.76,129.70,129.14,128.31,126.98,125.19$, 122.35, $110.34,76.85$

Optical Rotation: $[\alpha]_{\mathrm{D}}^{25}=-28.6(\mathrm{c}=0.24, \mathrm{MeOH})$. The absolute configuration was assigned by analog to that of $7 \mathbf{j}$.

96.5:3.5 er HPLC condition: Chiralpak AD-H, $n$-hexane $/ i-\mathrm{PrOH}=90: 10$, flow rate $=1 \mathrm{ml} / \mathrm{min}$, wavelength $=254 \mathrm{~nm}, t_{R}=22.670 \mathrm{~min}$ for minor isomer, $t_{R}=31.828 \mathrm{~min}$ for major isomer,
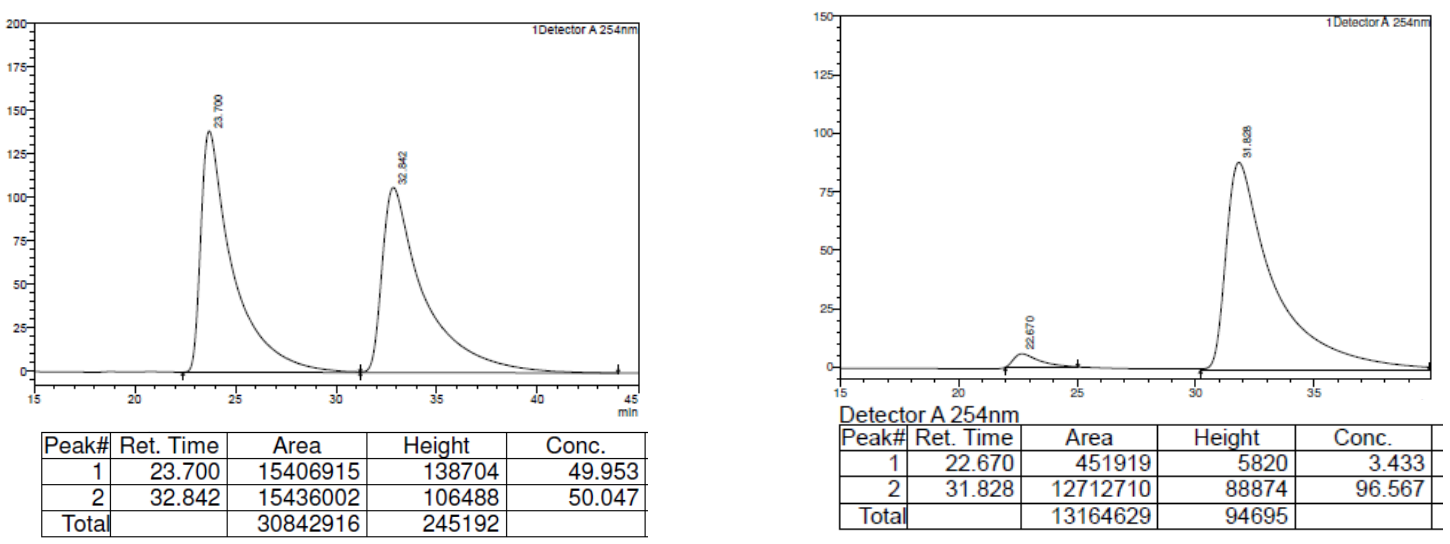

\section{(R, E)-3-hydroxy-1-methyl-3-styrylindolin-2-one}

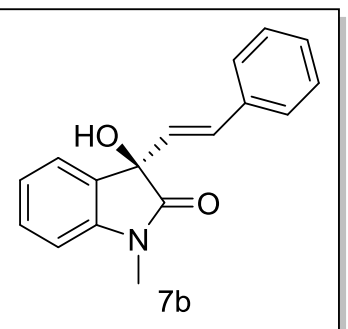

HRMS(ESI): $\mathrm{m} / \mathrm{z}$ Calcd. for $\left[\mathrm{C}_{17} \mathrm{H}_{15} \mathrm{NNaO}_{2}, \mathrm{M}+\mathrm{Na}\right]^{+}:$288.1000; Found: 288.0997.

Optical Rotation: $[\alpha]_{D}^{25}=-44.4(\mathrm{c}=0.37, \mathrm{MeOH})$, The absolute configuration was assigned by analog to that of $\mathbf{7} \mathbf{j}$.

95.5:4.5 er HPLC condition: Chiralcel $\mathrm{OJ}-\mathrm{H}, n$-hexane $/ i-\mathrm{PrOH}=90: 10$, flow rate $=1 \mathrm{ml} / \mathrm{min}$, wavelength $=254 \mathrm{~nm}, t_{R}=25.781 \mathrm{~min}$ for major isomer, $t_{R}=42.656 \min$ for minor isomer. 

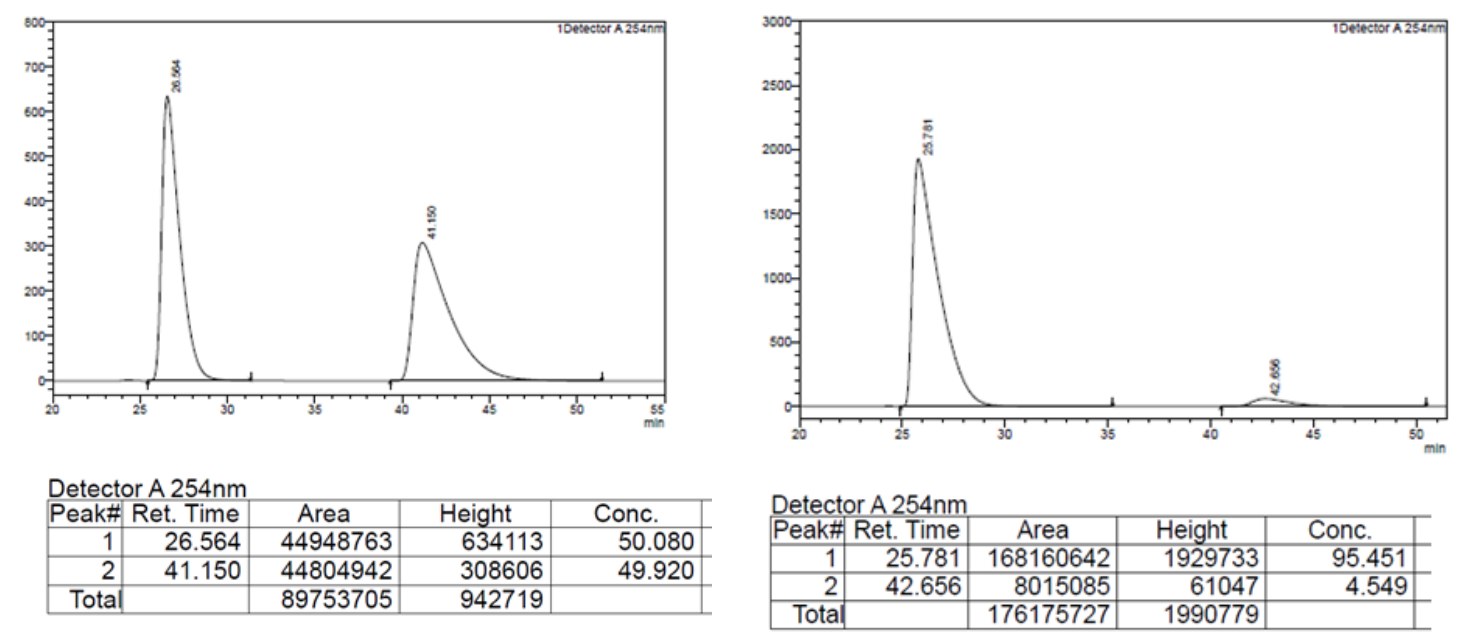

\section{(R, E)-1-benzyl-3-hydroxy-3-styrylindolin-2-one}

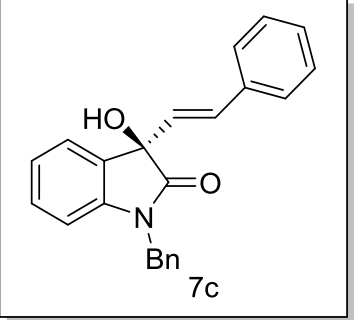

Pale yellow solid, $80 \%$ yield.

${ }^{1} \mathbf{H}$ NMR $\left(500 \mathrm{MHz}, \mathrm{CDCl}_{3}\right): \delta 7.40(\mathrm{~d}, J=6.8 \mathrm{~Hz}, 1 \mathrm{H}), 7.35-7.15$ $(\mathrm{m}, 11 \mathrm{H}), 7.06(\mathrm{t}, J=7.5 \mathrm{~Hz}, 1 \mathrm{H}), 6.71(\mathrm{~d}, J=16.0,1 \mathrm{H}), 6.71(\mathrm{~d}, J=$ $8.0,1 \mathrm{H}), 6.37(\mathrm{~d}, J=16.0 \mathrm{~Hz}, 1 \mathrm{H}), 4.96(\mathrm{~d}, J=15.7 \mathrm{~Hz}, 1 \mathrm{H}), 4.80(\mathrm{~d}$, $J=15.7 \mathrm{~Hz}, 1 \mathrm{H}), 3.39$ (s, 1H).

${ }^{13} \mathbf{C}$ NMR $\left(125 \mathrm{MHz}, \mathrm{CDCl}_{3}\right): \delta 176.93,142.39,135.85,135.31$, $131.85,129.84,129.52,128.86,128.56,128.23,127.73,127.31$, 127.16, 126.89, 124.83, 123.39, 109.79, 76.86, 43.91.

HRMS (ESI): $\mathrm{m} / \mathrm{z}$ Calcd. for $\left[\mathrm{C}_{23} \mathrm{H}_{19} \mathrm{NNaO}_{2}, \mathrm{M}+\mathrm{Na}\right]^{+}$: 364.1313 ; Found: 364.1317.

Optical Rotation: $[\alpha]_{\mathrm{D}}^{25}=-19.30(\mathrm{c}=0.38, \mathrm{MeOH})$. The absolute configuration was assigned by analog to that of $\mathbf{7 j}$.

93.5:6.5 er HPLC condition: Chiralcel OD-H, $n$-hexane $/ i-\operatorname{PrOH}=80: 20$, flow rate $=0.5 \mathrm{ml} / \mathrm{min}$, wavelength $=254 \mathrm{~nm}, \mathrm{t}_{\mathrm{R}}=21.603 \mathrm{~min}$ for minor isomer, $\mathrm{t}_{\mathrm{R}}=23.819$ min for major isomer.
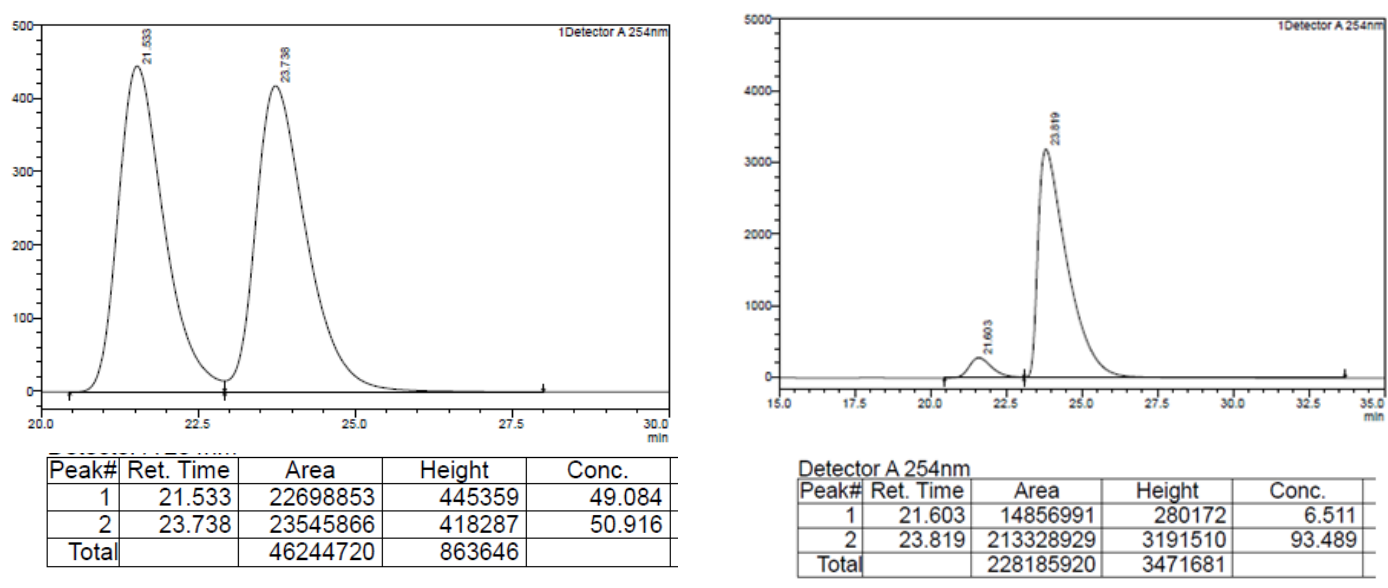


\section{(R, E)-3-hydroxy-1-(4-methoxybenzyl)-3-styrylindolin-2-one}

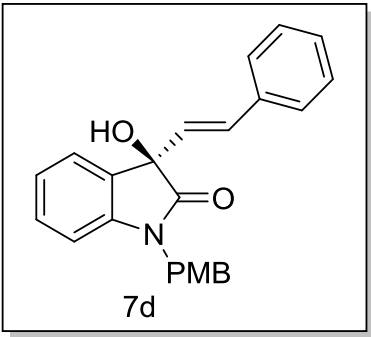

Brown solid, 70\% yield.

${ }^{1}$ H NMR (300 MHz, DMSO): $\delta 7.47-7.38(\mathrm{~m}, 2 \mathrm{H}), 7.37-7.18(\mathrm{~m}$, $7 \mathrm{H}), 7.06(\mathrm{t}, J=7.2 \mathrm{~Hz}, 1 \mathrm{H}), 6.99-6.84(\mathrm{~m}, 3 \mathrm{H}), 6.59(\mathrm{~d}, J=16.0$ $\mathrm{Hz}, 1 \mathrm{H}), 6.58(\mathrm{~s}, 1 \mathrm{H}), 6.40$ (d, J=16.0 Hz, 1H), 4.83 (s, 2H), 3.71 (s, $3 \mathrm{H})$.

${ }^{13}$ C NMR (75 MHz, DMSO): $\delta$ 176.57, 159.06, 142.45, 136.30, $131.59,130.16,129.66,129.45,129.14,128.56,128.39$, 127.02, 124.93, 123.07, 114.48, 109.94, 76.57, 55.51, 42.57.

HRMS (ESI): $\mathrm{m} / \mathrm{z}$ Calcd. for $\left[\mathrm{C}_{24} \mathrm{H}_{21} \mathrm{NNaO}_{3}, \mathrm{M}+\mathrm{Na}\right]^{+}:$394.1419; Found: 394.1420 .

Optical Rotation: $[\alpha]_{D}^{25}=-30.90(\mathrm{c}=0.36, \mathrm{MeOH})$. The absolute configuration was assigned by analog to that of $\mathbf{7 j}$.

93.5:6.5 er HPLC condition: Chiralcel OD-H, $n$-hexane $/ i$-PrOH $=80: 20$, flow rate $=1.0 \mathrm{ml} / \mathrm{min}$, wavelength $=254 \mathrm{~nm}, \mathrm{t}_{\mathrm{R}}=13.494 \mathrm{~min}$ for minor isomer, $\mathrm{t}_{\mathrm{R}}=16.522 \mathrm{~min}$ for major isomer.
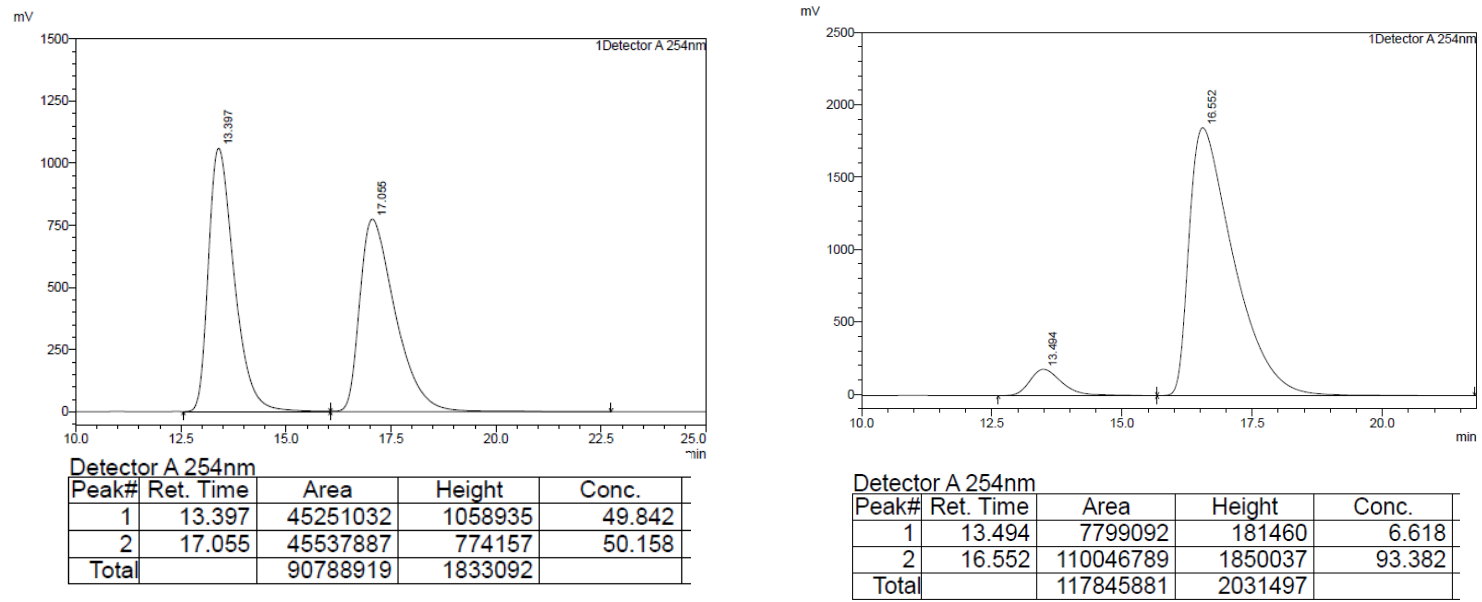

\section{(R, E)-3-hydroxy-5-methyl-3-styrylindolin-2-one}

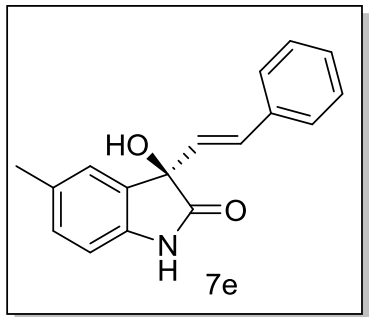

110.04, 76.92, 21.10.
Yellow solid, $50 \%$ yield.

${ }^{1}$ H NMR (300 MHz, DMSO): $\delta=10.27(\mathrm{~s}, 1 \mathrm{H}), 7.47-7.37(\mathrm{~m}, 2 \mathrm{H})$, $7.37-7.17(\mathrm{~m}, 3 \mathrm{H}), 7.12-7.00(\mathrm{~m}, 2 \mathrm{H}), 6.75(\mathrm{~d}, J=7.8,1 \mathrm{H}), 6.58(\mathrm{~d}$, $J=16.0,1 \mathrm{H}), 6.33(\mathrm{~s}, 1 \mathrm{H}), 6.32$ (d, $J=16.0,1 \mathrm{H}) 2.26$ (s, 3H).

${ }^{13}$ C NMR (75 MHz, DMSO): $\delta=178.27,139.54,136.46,132.29$, $131.20,129.86,129.83,129.67,129.11,128.25,126.96,125.75$,

HRMS (ESI): m/z Calcd. for $\left[\mathrm{C}_{17} \mathrm{H}_{15} \mathrm{NNaO}_{2}, \mathrm{M}+\mathrm{Na}\right]^{+}:$288.1000; Found: 288.1005.

Optical Rotation: $[\alpha]_{D}^{24}=-64.80(\mathrm{c}=0.10, \mathrm{MeOH})$, The absolute configuration was assigned by analog to that of $\mathbf{7 j}$. 
96:4 er. HPLC condition: Chiralcel AD-H, $n$-hexane $/ i-\mathrm{PrOH}=90: 10$, flow rate $=1 \mathrm{ml} / \mathrm{min}$, wavelength $=254 \mathrm{~nm}, t_{R}=19.402 \min$ for minor isomer, $t_{R}=27.085$ min for major isomer.
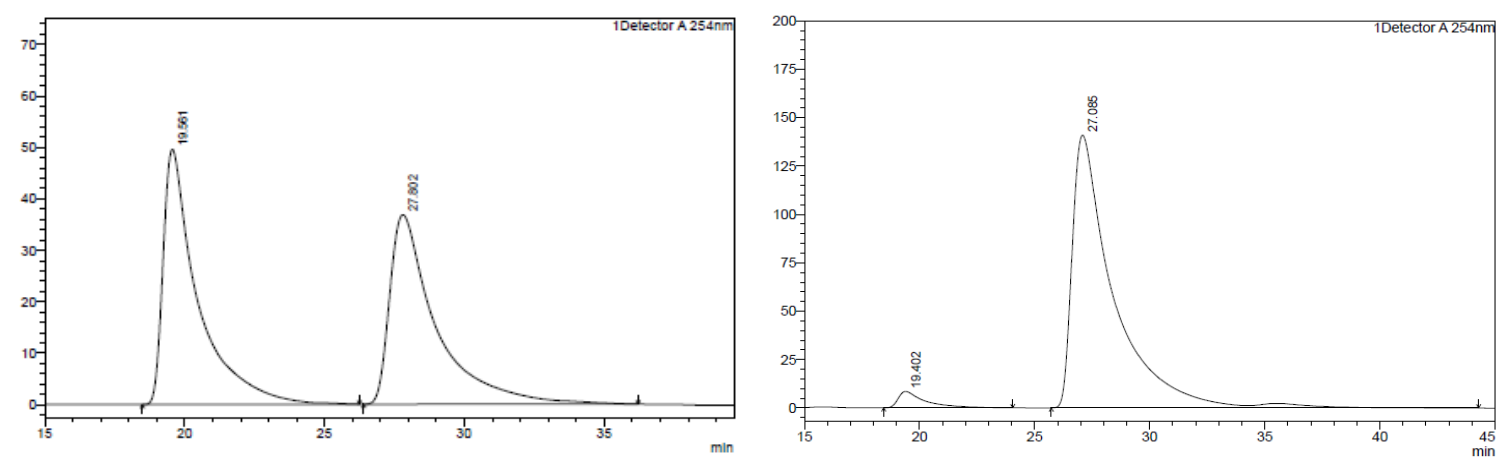

\begin{tabular}{|c|c|c|c|c|}
\hline \multirow{2}{*}{\multicolumn{2}{|c|}{$\begin{array}{l}\text { Detector A 254nm } \\
\text { Peak\# Ret. Time }\end{array}$}} & \multirow[b]{2}{*}{ Area } & \multirow[b]{2}{*}{ Height } & \multirow[b]{2}{*}{ Conc. } \\
\hline & & & & \\
\hline 1 & 19.561 & 4266581 & 49568 & 50.199 \\
\hline 2 & 27.802 & 4232717 & 36808 & 49.801 \\
\hline Tota & & 8499298 & 86376 & \\
\hline
\end{tabular}

Detector A $254 \mathrm{~nm}$
\begin{tabular}{|r|r|r|r|r|}
\hline Peak\# & Ret. Time & \multicolumn{1}{c|}{ Area } & Height & \multicolumn{1}{c|}{ Conc. } \\
\hline 1 & 19.402 & 690282 & 8475 & 3.770 \\
\hline 2 & 27.085 & 17619310 & 140881 & 96.230 \\
\hline Total & & 18309593 & 149356 & \\
\hline
\end{tabular}

\section{(R, E)-3-hydroxy-5-methoxy-3-styrylindolin-2-one}

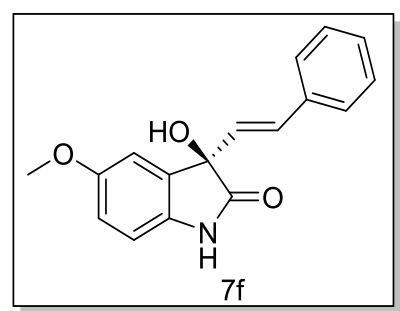

110.80, 77.25, 55.98.

Orange solid, $73 \%$ yield.

${ }^{1} \mathbf{H}$ NMR (300 MHz, DMSO): $\delta 10.21(\mathrm{~s}, 1 \mathrm{H}), 7.46-7.37$ (m, 2H), $7.36-7.17(\mathrm{~m}, 3 \mathrm{H}), 6.89(\mathrm{~d}, J=2.4 \mathrm{~Hz}, 1 \mathrm{H}), 6.85-6.73(\mathrm{~m}, 2 \mathrm{H})$, $6.56(\mathrm{~d}, J=16.0 \mathrm{~Hz}, 1 \mathrm{H}), 6.39(\mathrm{~s}, 1 \mathrm{H}), 6.35(\mathrm{~d}, J=16.0 \mathrm{~Hz}, 1 \mathrm{H})$, $3.71(\mathrm{~s}, 3 \mathrm{H})$.

${ }^{13}$ C NMR (75 MHz, DMSO): $\delta$ 178.18, 155.54, 136.45, 135.18, $133.43,129.89,129.81,129.13,128.31,127.01,114.53,111.82$,

HRMS (ESI): $\mathrm{m} / \mathrm{z}$ Calcd. for $\left[\mathrm{C}_{17} \mathrm{H}_{15} \mathrm{NNaO}_{3}, \mathrm{M}+\mathrm{Na}\right]^{+}$: 304.0950; Found: 304.0952.

Optical Rotation: $[\alpha]^{24}=-68.3(c=0.29, \mathrm{MeOH})$. The absolute configuration was assigned by analog to that of $\mathbf{7 j}$.

96:4 er. HPLC condition: Chiralpak AD-H, $n$-hexane $/ i-\operatorname{PrOH}=90: 10$, flow rate $=1 \mathrm{ml} / \mathrm{min}$, wavelength $=254 \mathrm{~nm}, t_{R}=29.988 \mathrm{~min}$ for minor isomer, $t_{R}=41.878$ min for major isomer. 

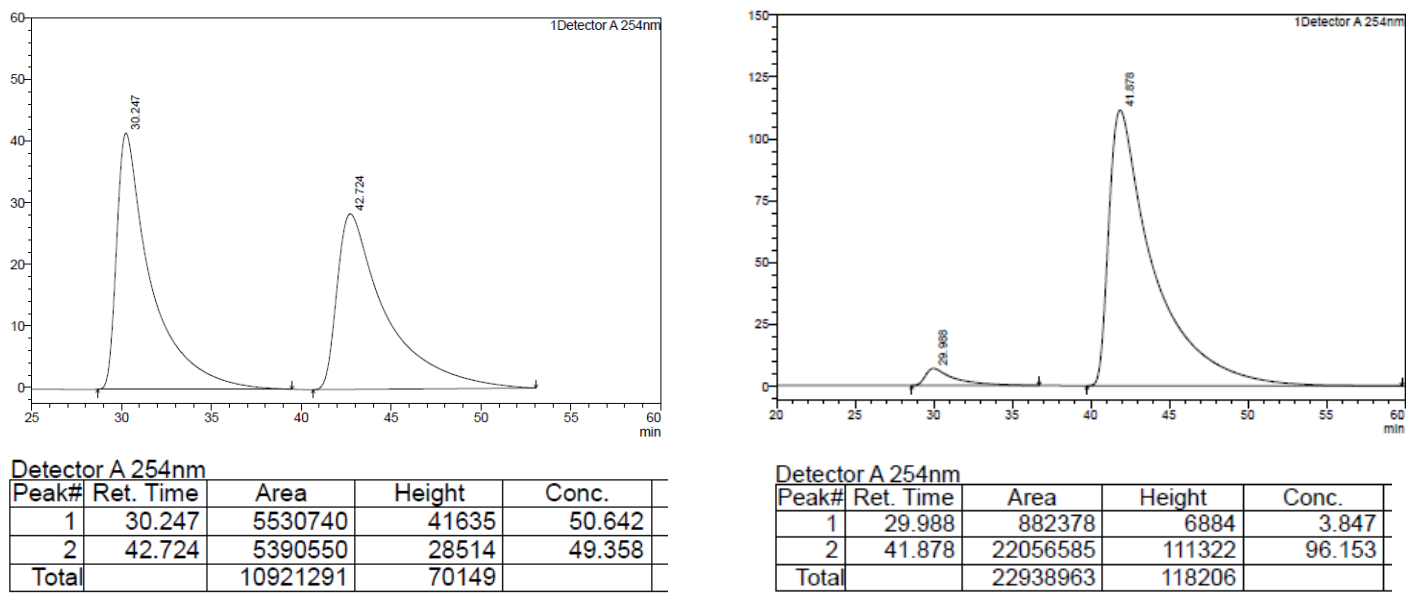

\begin{tabular}{|c|c|c|c|c|}
\hline \multicolumn{5}{|c|}{ PeakttRet Time } \\
\hline 1 & 29.988 & 882378 & 6884 & 3.847 \\
\hline 2 & 41.878 & 22056585 & 111322 & 96.153 \\
\hline Total & & 22938963 & 118206 & \\
\hline
\end{tabular}

\section{(R, E)-3-hydroxy-5-iodo-3-styrylindolin-2-one}

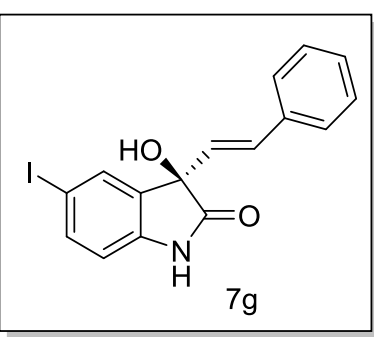

$85.11,76.69$.
Brown solid, $84 \%$ yield.

${ }^{1}$ H NMR (500 MHz, DMSO): $\delta 10.50(\mathrm{~s}, 1 \mathrm{H}), 7.59(\mathrm{dd}, J=8.1$, $1.8 \mathrm{~Hz}, 1 \mathrm{H}), 7.53(\mathrm{~d}, J=1.7 \mathrm{~Hz}, 1 \mathrm{H}), 7.46-7.41(\mathrm{~m}, 2 \mathrm{H}), 7.32$ (t, $J=7.5 \mathrm{~Hz}, 2 \mathrm{H}), 7.27-7.21(\mathrm{~m}, 1 \mathrm{H}), 6.72(\mathrm{~d}, J=8.1 \mathrm{~Hz}, 1 \mathrm{H})$, $6.59(\mathrm{~d}, J=16.0 \mathrm{~Hz}, 1 \mathrm{H}), 6.49(\mathrm{~s}, 1 \mathrm{H}), 6.35(\mathrm{~d}, J=16.0 \mathrm{~Hz}, 1 \mathrm{H})$. ${ }^{13}$ C NMR (125 MHz, DMSO): $\delta$ 177.54, 141.79, 138.17, 136.31, $135.05,133.34,130.14,129.12,129.04,128.40,127.09,112.92$,

HRMS (ESI): $\mathrm{m} / \mathrm{z}$ Calcd. for $\left[\mathrm{C}_{16} \mathrm{H}_{12} \mathrm{INNaO}_{2}, \mathrm{M}+\mathrm{Na}\right]^{+}$: 399.9810; Found: 399.9808.

Optical Rotation: $[\alpha]^{25}=-15.70(c=0.35, \mathrm{MeOH})$. The absolute configuration was assigned by analog to that of $\mathbf{7} \mathbf{j}$.

93.5:6.5 er. HPLC condition: Chiralpak AD-H, $n$-hexane $i-\mathrm{PrOH}=90: 10$, flow rate $=1$ $\mathrm{ml} / \mathrm{min}$, wavelength $=254 \mathrm{~nm}, \mathrm{t}_{\mathrm{R}}=19.938 \mathrm{~min}$ for minor isomer, $\mathrm{t}_{\mathrm{R}}=31.761 \mathrm{~min}$ for major isomer.
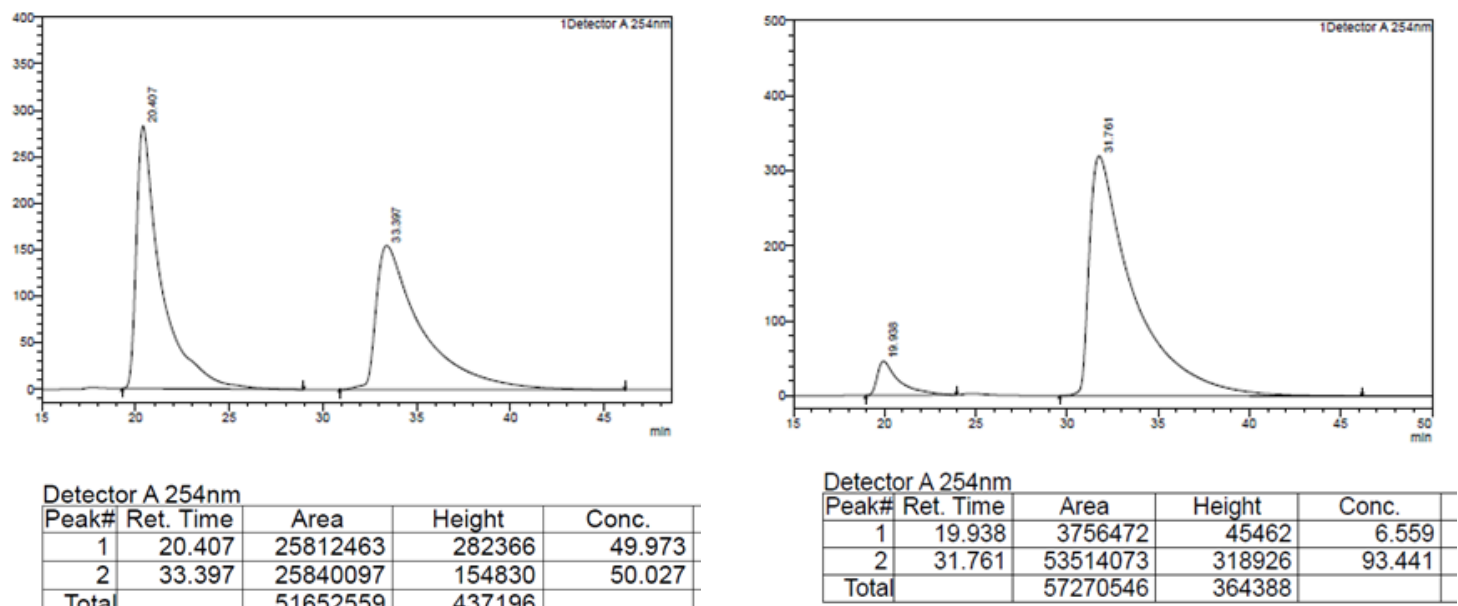


\section{(R, E)-5-chloro-3-hydroxy-3-styrylindolin-2-one}

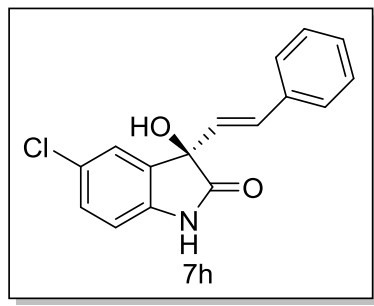

111.36, 76.36 .

Brown solid, $72 \%$ yield.

${ }^{1}$ H NMR (300 MHz, DMSO): $\delta 10.52(\mathrm{~s}, 1 \mathrm{H}), 7.51-7.39(\mathrm{~m}, 2 \mathrm{H})$, $7.38-7.18(\mathrm{~m}, 5 \mathrm{H}), 6.88(\mathrm{dd}, J=7.6,1.1 \mathrm{~Hz}, 1 \mathrm{H}), 6.59(\mathrm{~d}, J=$ $16.0 \mathrm{~Hz}, 1 \mathrm{H}), 6.53(\mathrm{~s}, 1 \mathrm{H}), 6.37(\mathrm{~d}, J=16.0 \mathrm{~Hz}, 1 \mathrm{H})$.

${ }^{13}$ C NMR (75 MHz, DMSO): $\delta$ 177.37, 140.39, 135.78, 133.83, $129.75,129.00,128.61,128.48,127.91,126.57,125.85,124.65$,

HRMS (ESI): $\mathrm{m} / \mathrm{z}$ Calcd. for $\left[\mathrm{C}_{16} \mathrm{H}_{12} \mathrm{ClNNaO}_{2}, \mathrm{M}+\mathrm{Na}\right]^{+}:$308.0454; Found: 308.0451.

Optical Rotation: $[\alpha]^{24}=-72.40(\mathrm{c}=0.29, \mathrm{MeOH})$. The absolute configuration was assigned by analog to that of $\mathbf{7} \mathbf{j}$.

93:7 er. HPLC condition: Chiralpak AD-H, $n$-hexane $/ i-\mathrm{PrOH}=90: 10$, flow rate $=1 \mathrm{ml} / \mathrm{min}$, wavelength $=254 \mathrm{~nm}, \mathrm{t}_{\mathrm{R}}=18.083 \mathrm{~min}$ for minor isomer, $\mathrm{t}_{\mathrm{R}}=25.527 \mathrm{~min}$ for major isomer.
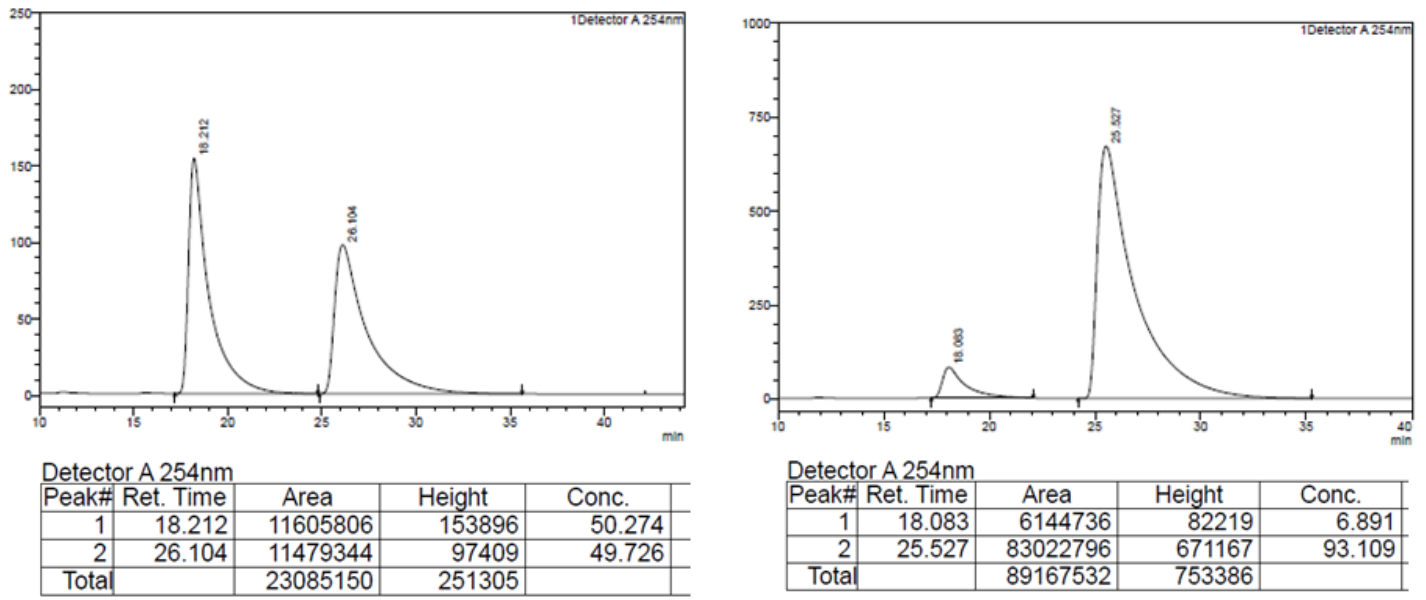

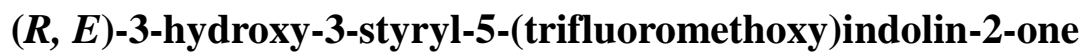

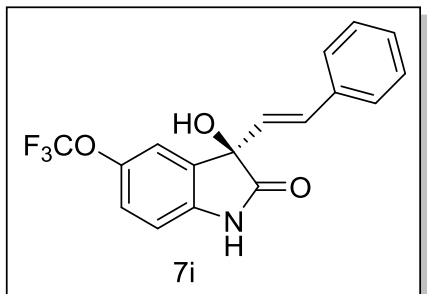

${ }^{19}$ F NMR (282 MHz, DMSO): $\delta$-57.24 (s, 3F).

Optical Rotation: $[\alpha]^{24}=-20.0(\mathrm{c}=0.51, \mathrm{MeOH})$. The absolute configuration was assigned by analogy to that of $\mathbf{7} \mathbf{j}$.

Gray solid, $90 \%$ yield.

${ }^{1}$ H NMR (300 MHz, DMSO): $\delta 10.58(\mathrm{~s}, 1 \mathrm{H}), 7.47-7.39(\mathrm{~m}$, $2 \mathrm{H}), 7.36-7.21(\mathrm{~m}, 5 \mathrm{H}), 6.95(\mathrm{~d}, J=9.1 \mathrm{~Hz}, 1 \mathrm{H}), 6.59(\mathrm{~d}, J=$ $16.0 \mathrm{~Hz}, 1 \mathrm{H}), 6.58(\mathrm{~s}, 1 \mathrm{H}), 6.38(\mathrm{~d}, J=16.0 \mathrm{~Hz}, 1 \mathrm{H})$.

${ }^{13}$ C NMR (75 MHz, DMSO): $\delta$ 178.04, 143.70, 141.08, 136.14, $133.84,130.31,129.03,128.72,128.35,126.97,122.86,120.57(\mathrm{q}$, $\left.J_{F C}=255.3 \mathrm{~Hz}\right), 118.62,111.20,76.80$.

HRMS (ESI): ) m/z Calcd. for $\left[\mathrm{C}_{17} \mathrm{H}_{12} \mathrm{~F}_{3} \mathrm{NNaO}_{3}, \mathrm{M}+\mathrm{Na}\right]^{+}$: 358.0667; Found: 358.0666. 
92:8 er. HPLC condition: Chiralpak AD-H, $n$-hexane $/ i-\mathrm{PrOH}=90: 10$, flow rate $=1 \mathrm{ml} / \mathrm{min}$, wavelength $=254 \mathrm{~nm}, \mathrm{t}_{\mathrm{R}}=11.056 \mathrm{~min}$ for minor isomer, $\mathrm{t}_{\mathrm{R}}=15.143 \mathrm{~min}$ for major isomer.
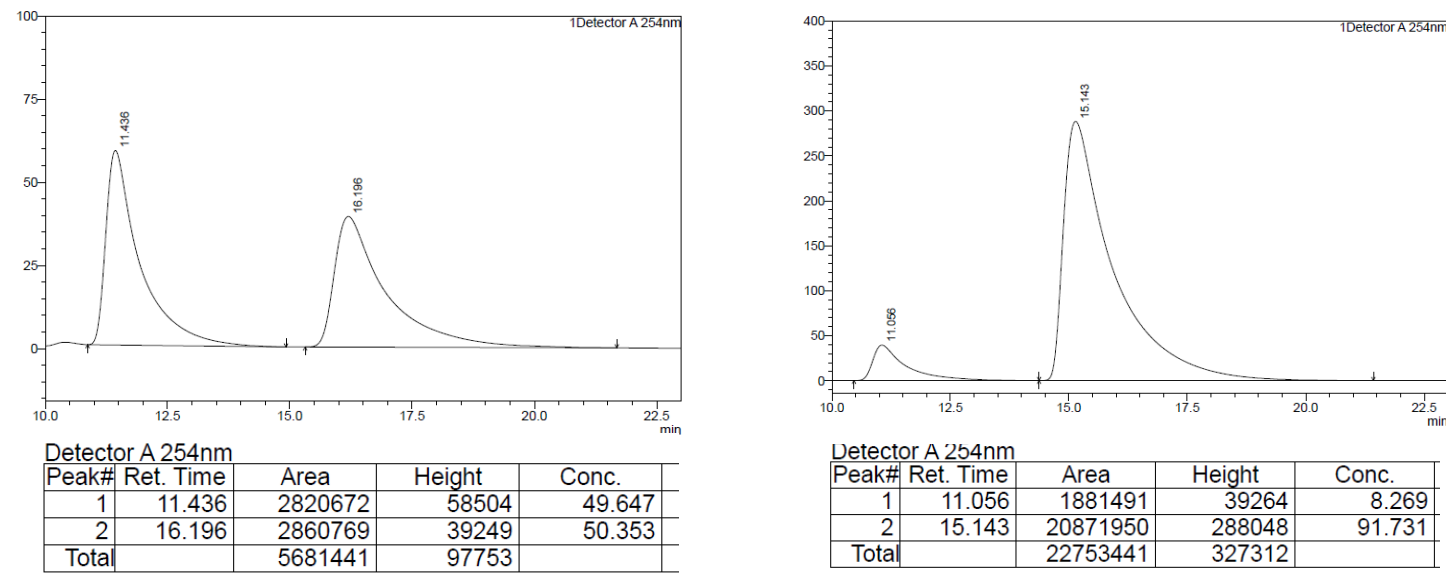

\section{(R, E)-5-chloro-3-hydroxy-1-(4-methoxybenzyl)-3-styrylindolin-2-one}

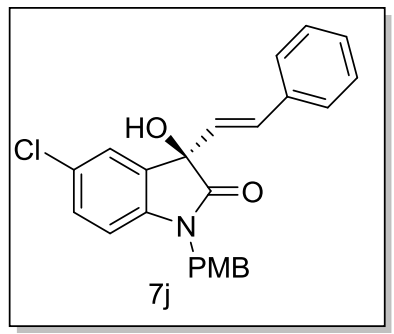

Known compound ${ }^{4}$, pale orange solid, yield $66 \%$.

${ }^{1} \mathbf{H}$ NMR $\left(500 \mathrm{MHz}, \mathrm{CDCl}_{3}\right): \delta 7.46-7.26(\mathrm{~m}, 6 \mathrm{H}), 7.23(\mathrm{~d}, J=$ $8.5 \mathrm{~Hz}, 3 \mathrm{H}), 6.87(\mathrm{~d}, J=8.6 \mathrm{~Hz}, 2 \mathrm{H}), 6.78(\mathrm{~d}, J=16.0 \mathrm{~Hz}, 1 \mathrm{H})$, $6.71(\mathrm{~d}, J=8.4 \mathrm{~Hz}, 1 \mathrm{H}), 6.37(\mathrm{~d}, J=16.0 \mathrm{~Hz}, 1 \mathrm{H}), 4.92(\mathrm{~d}, J=$ $15.5 \mathrm{~Hz}, 1 \mathrm{H}), 4.81(\mathrm{~d}, J=15.5 \mathrm{~Hz}, 1 \mathrm{H}), 3.80(\mathrm{~s}, 3 \mathrm{H}), 3.41(\mathrm{~s}$, $1 \mathrm{H})$.

${ }^{13}$ C NMR $\left(125 \mathrm{MHz}, \mathrm{CDCl}_{3}\right): \delta 176.47,159.30,140.89,135.60$, $132.25,131.23,129.73,128.86,128.64,128.57,128.47,126.96,126.89,126.65,125.33$, $114.38,110.88,76.81,55.28,43.58$.

Optical Rotation: $[\alpha]^{22}=-85.70\left(\mathrm{c}=0.40, \mathrm{CHCl}_{3}\right)$. The absolute configuration was assigned by the same compound reported in the literature ${ }^{[3]}$.

90:10 er. HPLC condition: Chiralcel OD-H, $n$-hexane $/ i-\mathrm{PrOH}=80: 20$, flow rate $=0.5 \mathrm{ml} / \mathrm{min}$, wavelength $=254 \mathrm{~nm}, t_{R}=23.937$ min for major isomer, $t_{R}=29.496$ min for minor isomer.
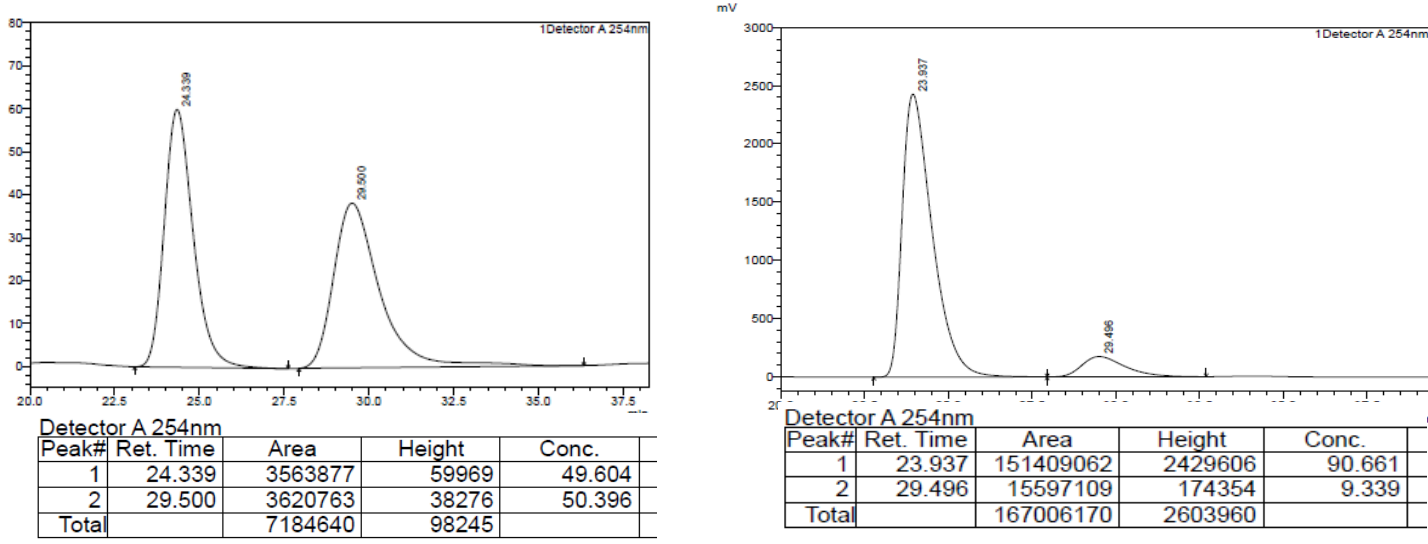


\section{$(R, E)$-7-chloro-3-hydroxy-3-styrylindolin-2-one}

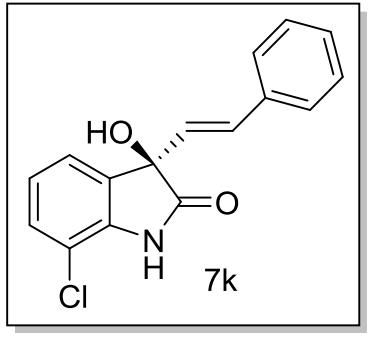

114.58, 77.40.

Brown solid, $90 \%$ yield.

${ }^{1}$ H NMR (500 MHz, DMSO): $\delta 10.82(\mathrm{~s}, 1 \mathrm{H}), 7.47-7.40(\mathrm{~m}, 2 \mathrm{H})$, $7.35-7.28(\mathrm{~m}, 3 \mathrm{H}), 7.24(\mathrm{t}, J=7.2 \mathrm{~Hz}, 2 \mathrm{H}), 7.04(\mathrm{dd}, J=8.0,7.5$ $\mathrm{Hz}, 1 \mathrm{H}), 6.58(\mathrm{~d}, J=16.0 \mathrm{~Hz}, 1 \mathrm{H}), 6.54(\mathrm{~s}, 1 \mathrm{H}), 6.36(\mathrm{~d}, J=16.0$ $\mathrm{Hz}, 1 \mathrm{H})$.

${ }^{13}$ C NMR (125 MHz, DMSO): $\delta$ 178.15, 139.74, 136.28, 134.14, $130.27,129.63,129.13,129.00,128.43,127.07,123.80,123.78$,

HRMS (ESI): $\mathrm{m} / \mathrm{z}$ Calcd. for $\left[\mathrm{C}_{16} \mathrm{H}_{12} \mathrm{ClNNaO}_{2}, \mathrm{M}+\mathrm{Na}\right]^{+}: 308.0454$; Found: 308.0455 .

Optical Rotation: $[\alpha]_{D}^{24}=-26.00(\mathrm{c}=0.10, \mathrm{MeOH})$. The absolute configuration was assigned by analog to that of $\mathbf{7 j}$.

92:8 er. HPLC condition: Chiralcel OD-H, $n$-hexane $/ i-\mathrm{PrOH}=80: 20$, flow rate $=1 \mathrm{ml} / \mathrm{min}$, wavelength $=254 \mathrm{~nm}, \mathrm{t}_{\mathrm{R}}=7.379 \mathrm{~min}$ for minor isomer, $\mathrm{t}_{\mathrm{R}}=10.816 \mathrm{~min}$ for major isomer.
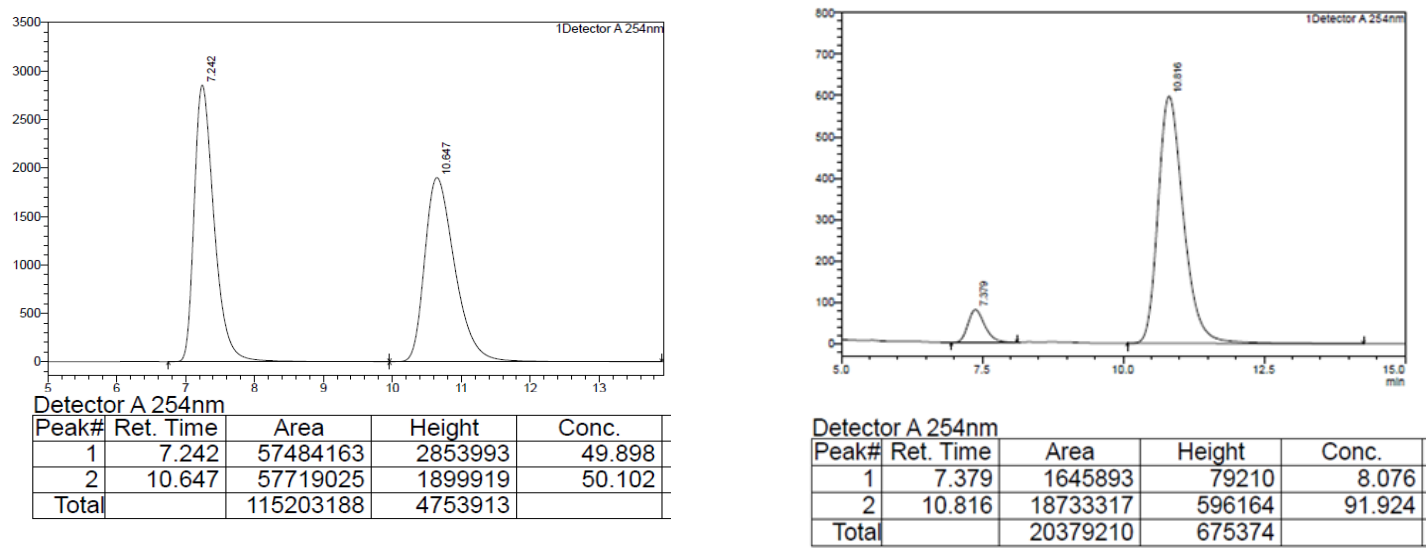

\section{(R, E)-3-(4-chlorostyryl)-3-hydroxyindolin-2-one}

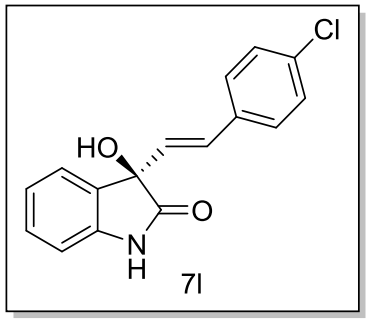

Pale yellow solid, yield 55\%.

${ }^{1}$ H NMR (300 MHz, DMSO): $\delta 10.38(\mathrm{~s}, 1 \mathrm{H}), 7.55-7.16(\mathrm{~m}, 6 \mathrm{H})$, $7.00(\mathrm{t}, J=7.0 \mathrm{~Hz}, 1 \mathrm{H}), 6.86(\mathrm{~d}, J=7.3 \mathrm{~Hz}, 1 \mathrm{H}), 6.58(\mathrm{~d}, J=16.0$ $\mathrm{Hz}, 1 \mathrm{H}), 6.40(\mathrm{~s}, 1 \mathrm{H}), 6.37(\mathrm{~d}, J=16.0 \mathrm{~Hz}, 1 \mathrm{H})$.

${ }^{13}$ C NMR (75 MHz, DMSO): $\delta 178.01,141.95,135.33,132.53$, $131.98,130.57,129.63,128.98,128.62,128.47,125.07,122.26$, $110.24,76.70$.

HRMS (ESI): ) m/z Calcd. for $\left[\mathrm{C}_{16} \mathrm{H}_{12} \mathrm{ClNNaO}_{2}, \mathrm{M}+\mathrm{Na}\right]^{+}:$308.0454; Found: 308.0452.

Optical Rotation: $[\alpha]^{25}=-18.20(\mathrm{c}=0.15, \mathrm{MeOH})$. The absolute configuration was assigned by analog to that of $\mathbf{7} \mathbf{j}$.

95:5 er. HPLC condition: Chiralcel AD-H, $n$-hexane $/ i-\mathrm{PrOH}=90: 10$, flow rate $=1.0 \mathrm{ml} / \mathrm{min}$, wavelength $=254 \mathrm{~nm}, \mathrm{t}_{\mathrm{R}}=28.185 \mathrm{~min}$ for minor isomer, $\mathrm{t}_{\mathrm{R}}=38.120$ min for major isomer. 

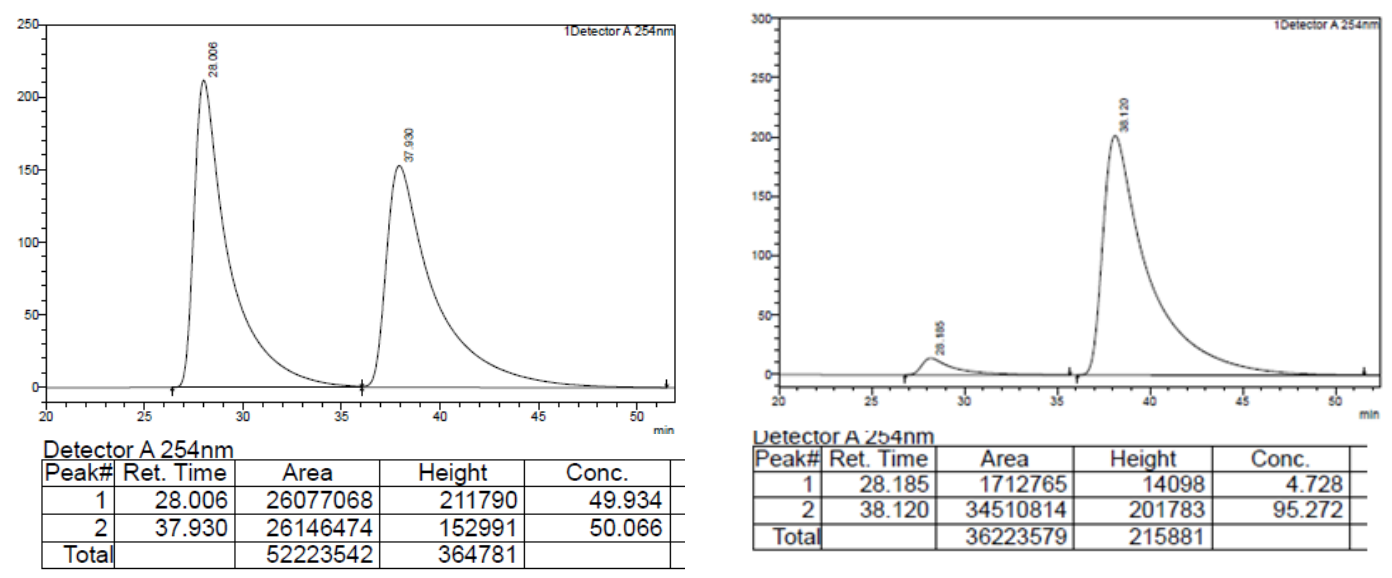

\section{(R, E)-3-(4-fluorostyryl)-3-hydroxyindolin-2-one}

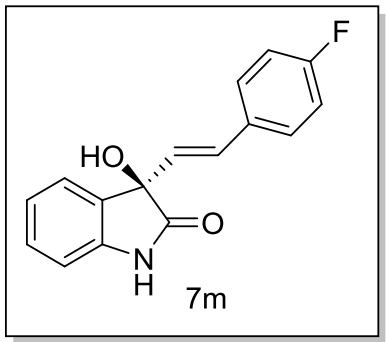

Pale yellow solid, yield $80 \%$.

${ }^{1}$ H NMR (300 MHz, DMSO): $\delta 10.36(\mathrm{~s}, 1 \mathrm{H}), 7.53-7.41(\mathrm{~m}$, $2 \mathrm{H}), 7.31-7.05(\mathrm{~m}, 4 \mathrm{H}), 7.05-6.94(\mathrm{~m}, 1 \mathrm{H}), 6.89-6.80(\mathrm{~m}$, $1 \mathrm{H}), 6.55(\mathrm{~d}, J=16.0 \mathrm{~Hz}, 1 \mathrm{H}), 6.36(\mathrm{~s}, 1 \mathrm{H}), 6.30(\mathrm{~d}, J=16.0 \mathrm{~Hz}$, $1 \mathrm{H})$.

${ }^{13}$ C NMR (75 MHz, DMSO): $\delta$ 178.11, $162.08\left(\mathrm{~d}, J_{F C}=245.0\right.$ $\mathrm{Hz}), 141.95,132.93\left(\mathrm{~d}, J_{F C C C C}=3.1 \mathrm{~Hz}\right), 132.08,129.58,129.53$, $128.83\left(\mathrm{~d}, J_{F C C C}=8.1 \mathrm{~Hz}\right), 128.57,125.06,122.23,115.83\left(\mathrm{~d}, J_{F C C}=21.5 \mathrm{~Hz}\right), 110.21,76.67$. ${ }^{19}$ F NMR (282 MHz, DMSO): $\delta-114.21(\mathrm{tt}, J=9.0,5.6 \mathrm{~Hz}, 1 \mathrm{~F})$.

HRMS (ESI): $\mathrm{m} / \mathrm{z}$ Calcd. for $\left[\mathrm{C}_{16} \mathrm{H}_{12} \mathrm{FNNaO}_{2}, \mathrm{M}+\mathrm{Na}\right]^{+}: 292.0750$; Found: 292.0752. Optical Rotation: $[\alpha]_{D}^{25}=-30.33(\mathrm{c}=0.17, \mathrm{MeOH})$. The absolute configuration was assigned by analog to that of $\mathbf{7 j}$.

95.5:4.5 er. HPLC condition: Chiralpak AD-H, $n$-hexane $/ i-\mathrm{PrOH}=90: 10$, flow rate $=1.0$ $\mathrm{ml} / \mathrm{min}$, wavelength $=254 \mathrm{~nm}, \mathrm{t}_{\mathrm{R}}=24.103 \mathrm{~min}$ for minor isomer, $\mathrm{t}_{\mathrm{R}}=34.289$ min for major isomer.
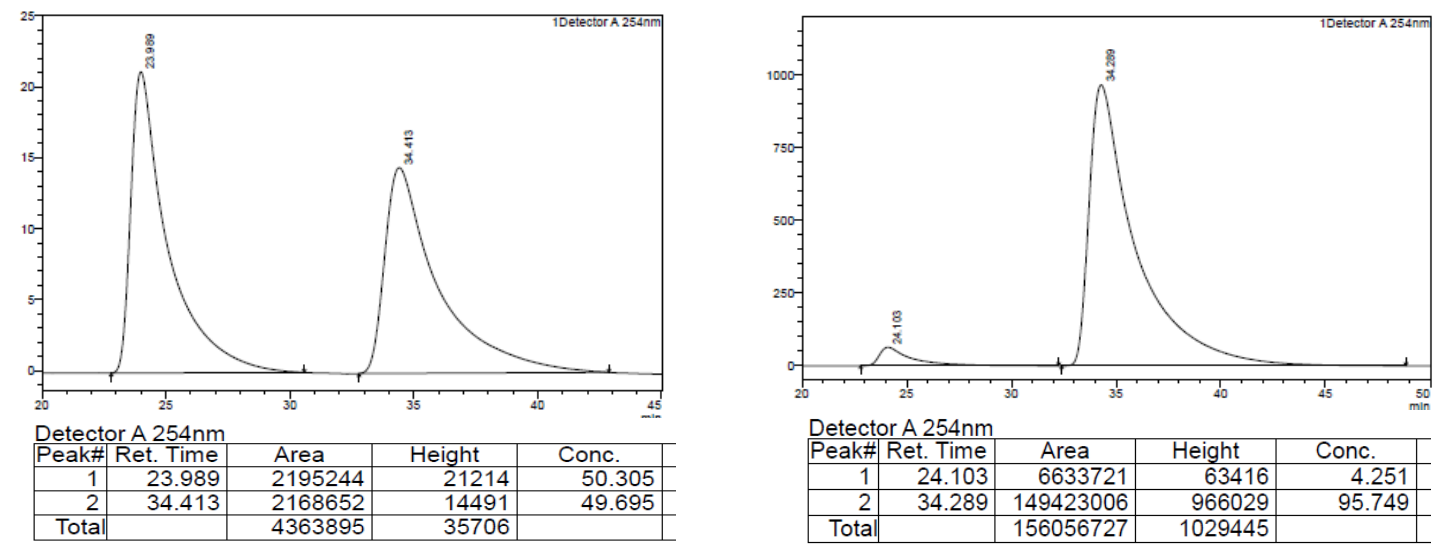


\section{(R, E)-3-hydroxy-3-(4-methylstyryl)indolin-2-one}

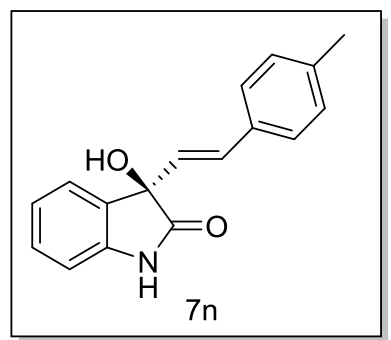

Pale yellow solid, yield: $78 \%$.

${ }^{1}$ H NMR (300 MHz, DMSO): $\delta 10.34(\mathrm{~s}, 1 \mathrm{H}), 7.36$ - 7.17 (m, $4 \mathrm{H}), 7.12(\mathrm{~d}, J=8.0 \mathrm{~Hz}, 2 \mathrm{H}), 7.00(\mathrm{td}, J=7.7,1.0 \mathrm{~Hz}, 1 \mathrm{H}), 6.86$ $(\mathrm{d}, J=7.6 \mathrm{~Hz}, 1 \mathrm{H}), 6.49(\mathrm{~d}, J=16.0 \mathrm{~Hz}, 1 \mathrm{H}), 6.33(\mathrm{~s}, 1 \mathrm{H}), 6.28$ (d, $J=16.0 \mathrm{~Hz}, 1 \mathrm{H}), 2.27$ (s, 3H).

${ }^{13}$ C NMR (75 MHz, DMSO): $\delta$ 178.24, 141.93, 137.56, 133.54, $132.14,129.69,129.61,129.53,128.60,126.80,125.07,122.20$,

110.19, 76.70, 21.12 .

HRMS (ESI): $\mathrm{m} / \mathrm{z}$ Calcd. for $\left[\mathrm{C}_{17} \mathrm{H}_{16} \mathrm{NNaO}_{2}, \mathrm{M}+\mathrm{Na}\right]^{+}:$: 288.1000; Found: 288.0997.

Optical Rotation: $[\alpha]_{\mathrm{D}}^{25}=-36.50(\mathrm{c}=0.28, \mathrm{MeOH})$. The absolute configuration was assigned by analog to that of $\mathbf{7} \mathbf{j}$.

95:5 er. HPLC condition: Chiralcel AD-H, $n$-hexane $/ i-\mathrm{PrOH}=90: 10$, flow rate $=1.0 \mathrm{ml} / \mathrm{min}$, wavelength $=254 \mathrm{~nm}, t_{R}=25.116$ min for minor isomer, $t_{R}=34.343$ min for major isomer.
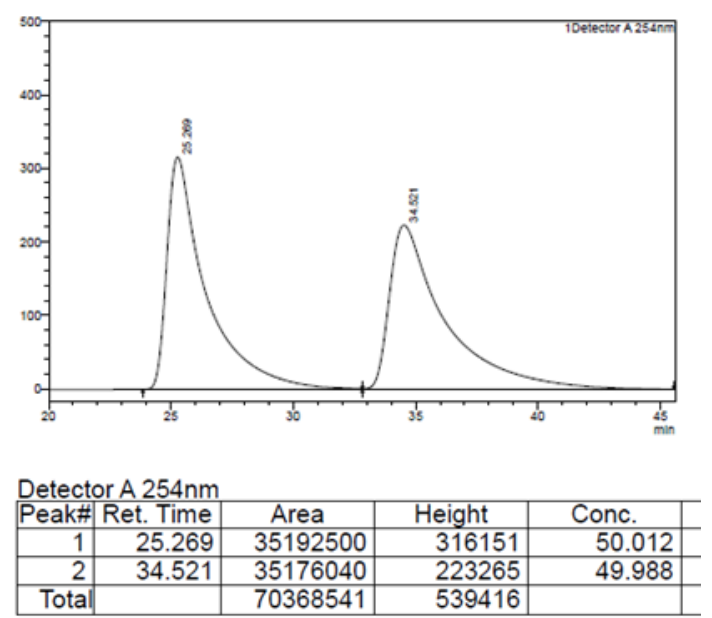
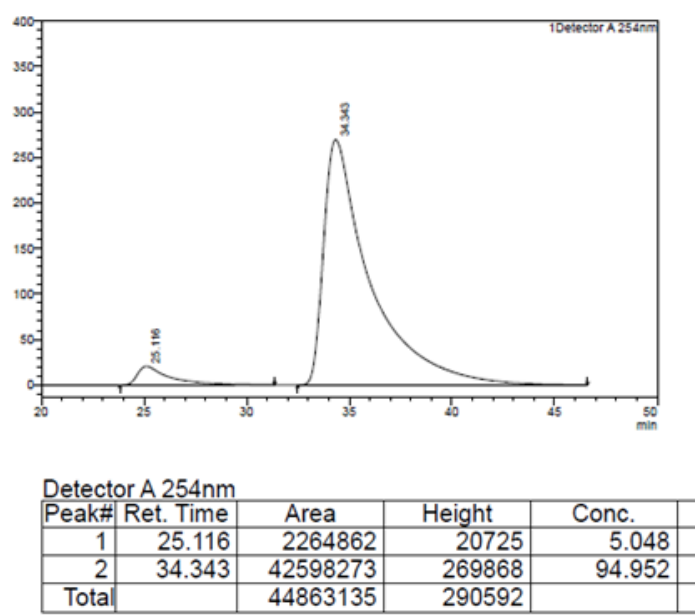

\section{$(R, E)$-3-hydroxy-3-(prop-1-en-1-yl)indolin-2-one}

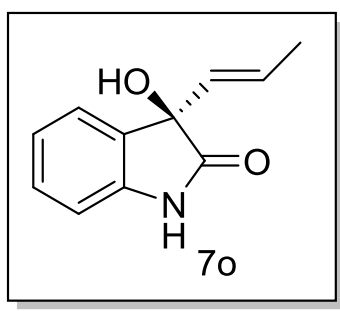

Pale yellow solid, yield: $65 \%$.

${ }^{1}$ H NMR (500 MHz, DMSO): $\delta 10.22(\mathrm{~s}, 1 \mathrm{H}), 7.28-7.14(\mathrm{~m}, 2 \mathrm{H})$, $6.98(\mathrm{td}, J=7.5,0.9 \mathrm{~Hz}, 1 \mathrm{H}), 6.82(\mathrm{~d}, J=7.7 \mathrm{~Hz}, 1 \mathrm{H}), 6.08(\mathrm{~s}, 1 \mathrm{H})$, $5.60(\mathrm{dd}, J=15.4,1.4 \mathrm{~Hz}, 1 \mathrm{H}), 5.50(\mathrm{dq}, J=15.4,6.4 \mathrm{~Hz}, 1 \mathrm{H}), 1.64$ (dd, $J=6.4,1.3 \mathrm{~Hz}, 3 \mathrm{H})$.

${ }^{13}$ C NMR (125 MHz, DMSO): $\delta$ 178.69, 141.96, 132.31, 131.63, $129.39,126.57,125.10,122.08,110.13,76.45,17.85$.

HRMS (ESI): $\mathrm{m} / \mathrm{z}$ Calcd. for $\left[\mathrm{C}_{11} \mathrm{H}_{11} \mathrm{NNaO}_{2}, \mathrm{M}+\mathrm{Na}\right]^{+}:$: 212.0687; Found: 212.0690 .

Optical Rotation: $[\alpha]_{D}^{25}=13.00(c=0.10$, Ethyl acetate), The absolute configuration was assigned by analog to that of $\mathbf{7} \mathbf{j}$.

95:5 er. HPLC condition: Chiralcel OD-H, $n$-hexane $/ i-\mathrm{PrOH}=90: 10$, flow rate $=1.0 \mathrm{ml} / \mathrm{min}$, wavelength $=254 \mathrm{~nm}, \mathrm{t}_{\mathrm{R}}=11.535 \mathrm{~min}$ for minor isomer, $\mathrm{t}_{\mathrm{R}}=15.126$ min for major isomer. 

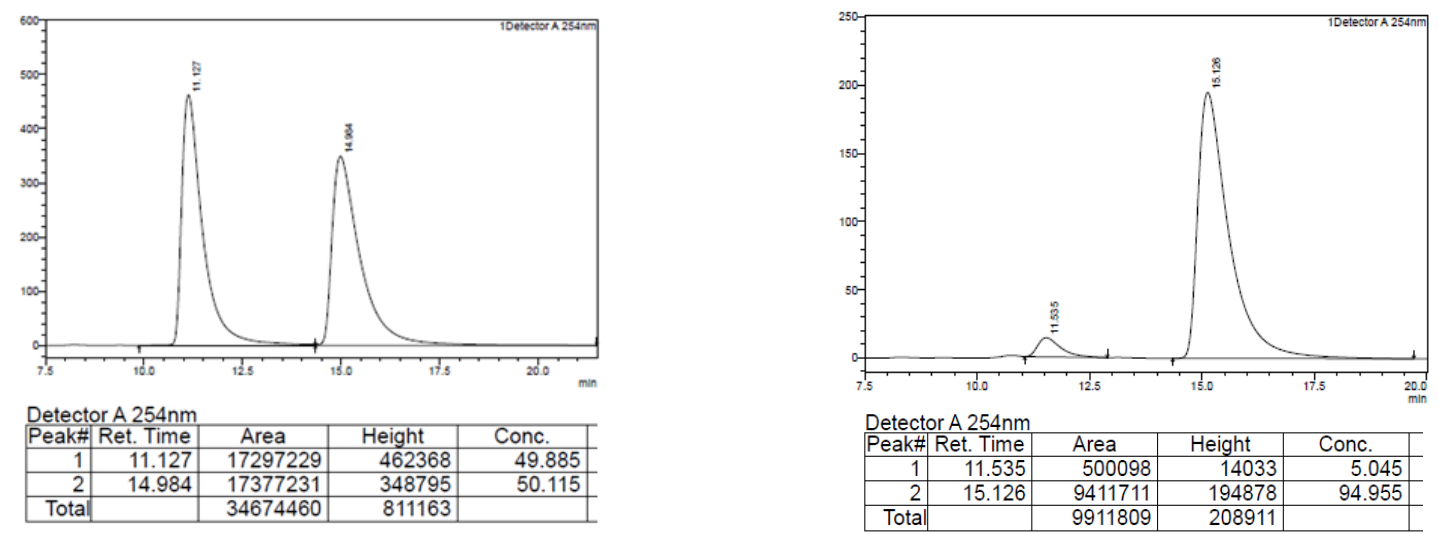

\section{(S)-3-hydroxy-3-(2-methylprop-1-en-1-yl)indolin-2-one}

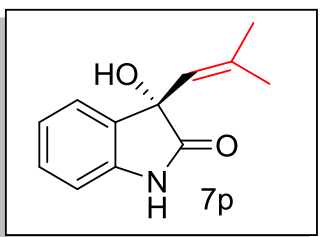

Brown soild, yield: $80 \%$.

${ }^{1}$ H NMR (500 MHz, DMSO): $\delta 10.24(\mathrm{~s}, 1 \mathrm{H}), 7.25-7.09$ (m, 2H), $6.95(\mathrm{t}, \mathrm{J}=7.5 \mathrm{~Hz}, 1 \mathrm{H}), 6.80(\mathrm{~d}, \mathrm{~J}=7.6 \mathrm{~Hz}, 1 \mathrm{H}), 6.01(\mathrm{~s}, 1 \mathrm{H}), 5.39(\mathrm{~s}$, $1 \mathrm{H}), 1.66(\mathrm{~s}, 3 \mathrm{H}), 1.35(\mathrm{~s}, 3 \mathrm{H})$.

${ }^{13} \mathbf{C}$ NMR $\left(75 \mathrm{MHz}, \mathrm{CDCl}_{3}\right): \delta 178.81,142.15,135.12,134.16$, $129.32,127.15,124.80,122.28,109.96,75.70,27.00,18.71$.

HRMS (ESI): $\mathrm{m} / \mathrm{z}$ Calcd. for $\left[\mathrm{C}_{12} \mathrm{H}_{13} \mathrm{NNaO}_{2}, \mathrm{M}-\mathrm{H}\right]$ ]: 202.0874; Found: 202.0871.

Optical Rotation: $[\alpha]_{D}^{25}=-18.5(c=0.23, \mathrm{MeOH})$. The absolute configuration was assigned by analog to that of $\mathbf{7 j}$.

97:3 er. HPLC condition: Chiralpak IE column, n-hexane/i-PrOH $=80: 20$, flow rate $=1$ $\mathrm{ml} / \mathrm{min}$, wavelength $=254 \mathrm{~nm}, \mathrm{t}_{\mathrm{R}}=8.716 \mathrm{~min}$ for minor isomer, $\mathrm{t}_{\mathrm{R}}=9.398$ min for major.
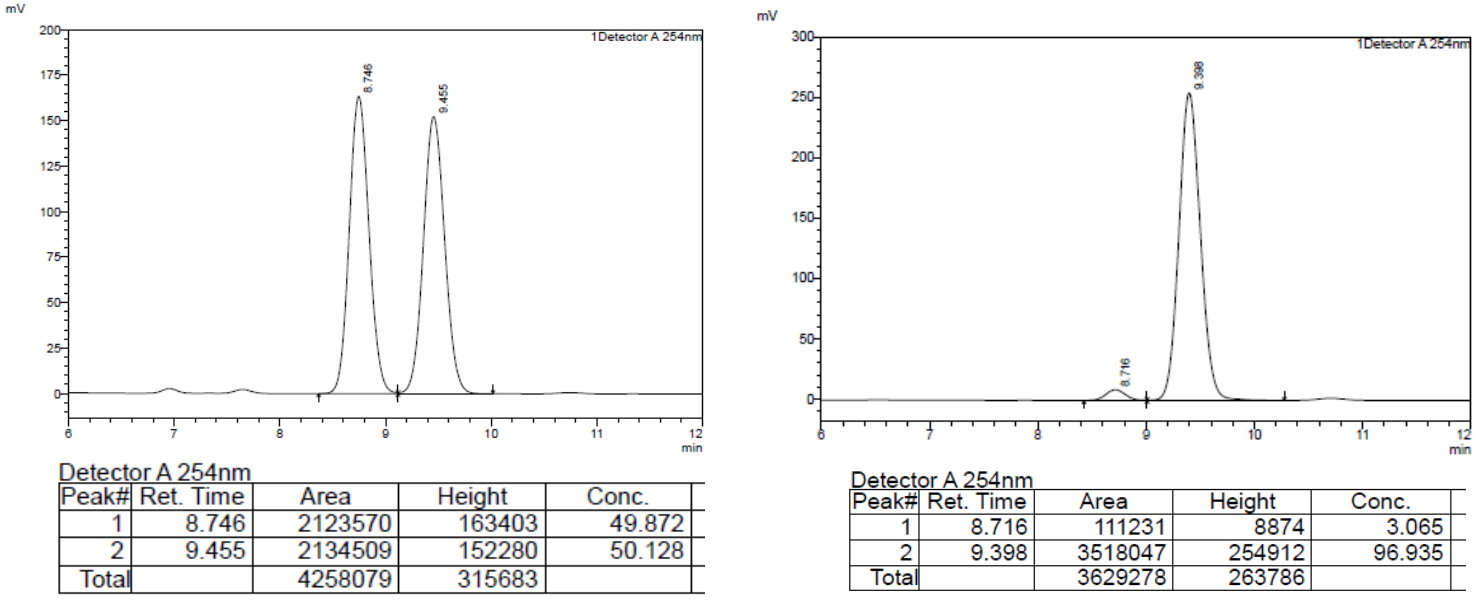


\section{Analytical data of vinylation products 14}

\section{(R, E)-4-styryl-3, 4-dihydrobenzo[1, 2, 3]oxathiazine 2, 2-dioxide}

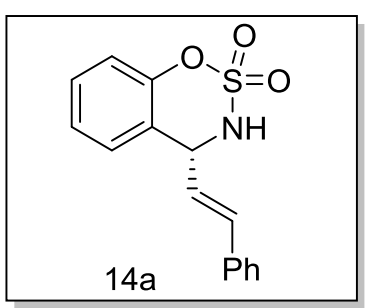

Colorless solid, $75 \%$ Yield.

${ }^{1} \mathbf{H}$ NMR $\left(500 \mathrm{MHz}, \mathrm{CDCl}_{3}\right): \delta 7.53-7.33(\mathrm{~m}, 6 \mathrm{H}), 7.31-7.17$ (m, 2H), $7.05(\mathrm{~d}, \mathrm{~J}=8.3 \mathrm{~Hz}, 1 \mathrm{H}), 6.88(\mathrm{~d}, \mathrm{~J}=15.7 \mathrm{~Hz}, 1 \mathrm{H}), 6.32$ (dd, J = 15.7, 8.6 Hz, 1H), 5.47 (t, J = 7.4 Hz, 1H), 4.93 (s, 1H). ${ }^{13}$ C NMR (125MHz, $\left.\mathrm{CDCl}_{3}\right): \delta 151.00,136.76,135.26,129.85$, $128.91,128.84,127.84,126.96,125.37,124.16,121.30,118.88$, 60.03.

HRMS (ESI): m/z Calcd. for $\left[\mathrm{C}_{15} \mathrm{H}_{12} \mathrm{NO}_{3} \mathrm{~S}, \mathrm{M}-\mathrm{H}\right]=286.0543$; Found: 286.0542.

Optical Rotation: $[\alpha]^{22}=-3.70\left(\mathrm{c}=0.40, \mathrm{CH}_{2} \mathrm{Cl}_{2}\right)$. The absolute configuration was assigned according the analogue 14k.

99.8 : 0.2 er. HPLC condition: Chiralcel OD-H column, $n$-hexane $/ i-\mathrm{PrOH}=80: 20$, flow rate $=$ $0.8 \mathrm{ml} / \mathrm{min}$, wavelength $=254 \mathrm{~nm}, t_{R}=23.294 \mathrm{~min}$ for major isomer, $t_{R}=29.770 \min$ for minor isomer.
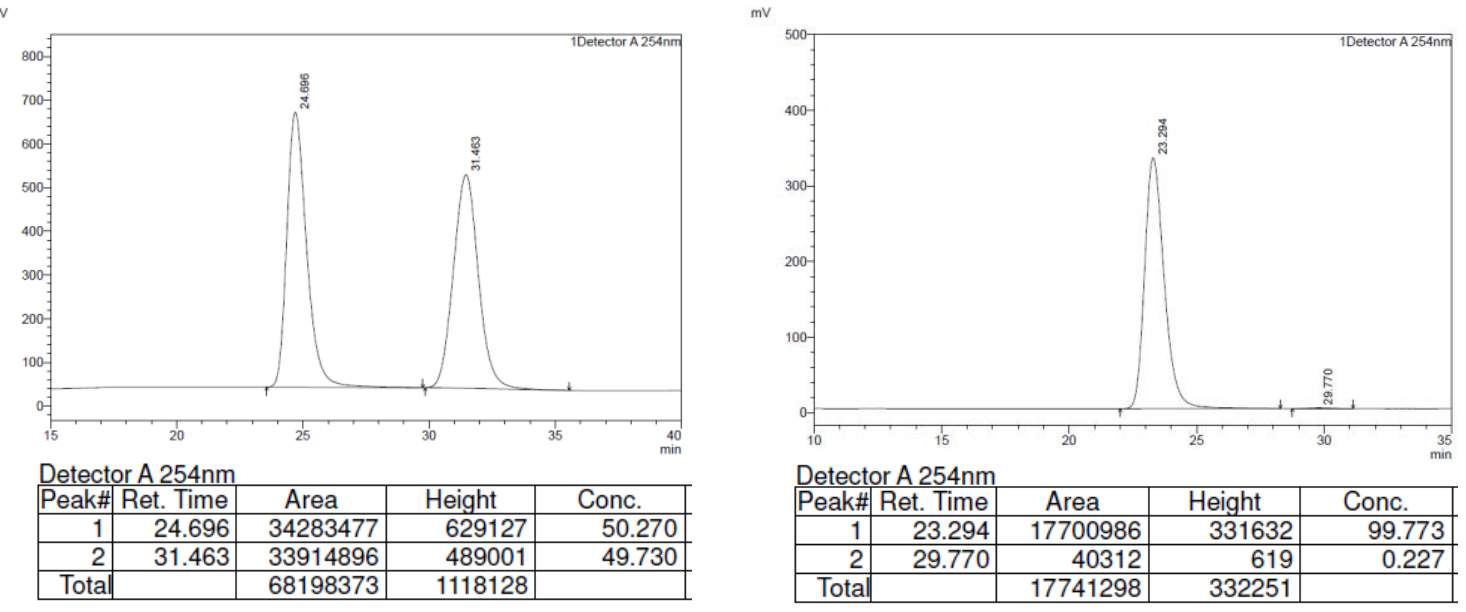

(R, E)-6-methoxy-4-styryl-3, 4-dihydrobenzo[e][1, 2, 3]oxathiazine 2, 2-dioxide

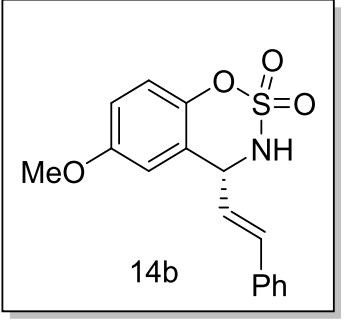

Colorless solid, $73 \%$ yield.

${ }^{1} \mathbf{H}$ NMR $\left(500 \mathrm{MHz}, \mathrm{CDCl}_{3}\right): \delta 7.46(\mathrm{~d}, \mathrm{~J}=7.2 \mathrm{~Hz}, 2 \mathrm{H}), 7.42-7.32$ $(\mathrm{m}, 2 \mathrm{H}), 7.01(\mathrm{~d}, \mathrm{~J}=9.1 \mathrm{~Hz}, 1 \mathrm{H}), 6.93-6.81(\mathrm{~m}, 2 \mathrm{H}), 6.73(\mathrm{~d}, \mathrm{~J}=$ $2.9 \mathrm{~Hz}, 1 \mathrm{H}), 6.31(\mathrm{dd}, \mathrm{J}=15.7,8.6 \mathrm{~Hz}, 1 \mathrm{H}), 5.43(\mathrm{t}, \mathrm{J}=8.3 \mathrm{~Hz}, 1 \mathrm{H})$, $4.82(\mathrm{~d}, \mathrm{~J}=8.1 \mathrm{~Hz}, 1 \mathrm{H}), 3.78(\mathrm{~s}, 3 \mathrm{H})$.

${ }^{13} \mathrm{C}$ NMR $\left(125 \mathrm{MHz}, \mathrm{CDCl}_{3}\right): \delta 156.76,144.74,136.83,135.22$, 
$128.92,128.83,126.96,124.12,122.16,119.80,115.13,112.69,60.12,55.79$.

HRMS (ESI): $\mathrm{m} / \mathrm{z}$ Calcd. For $\left[\mathrm{C}_{16} \mathrm{H}_{15} \mathrm{NNaO}_{4} \mathrm{~S}, \mathrm{M}+\mathrm{Na}\right]^{+}: 340.0614$; Found: 340.0614.

Optical Rotation: $[\alpha]_{D}^{22}=-15.67\left(c=0.50, \mathrm{CH}_{2} \mathrm{Cl}_{2}\right)$. The absolute configuration was assigned according the analogue 14k.

99.8: 0.2 er. HPLC condition: ChiralcelOD-H column, $n$-hexane $/ i-\operatorname{PrOH}=80: 20$, flow rate $=$ $0.8 \mathrm{ml} / \mathrm{min}$, wavelength $=254 \mathrm{~nm}, t_{R}=30.949 \min$ for major isomer, $t_{R}=39.189 \min$ for minor isomer.
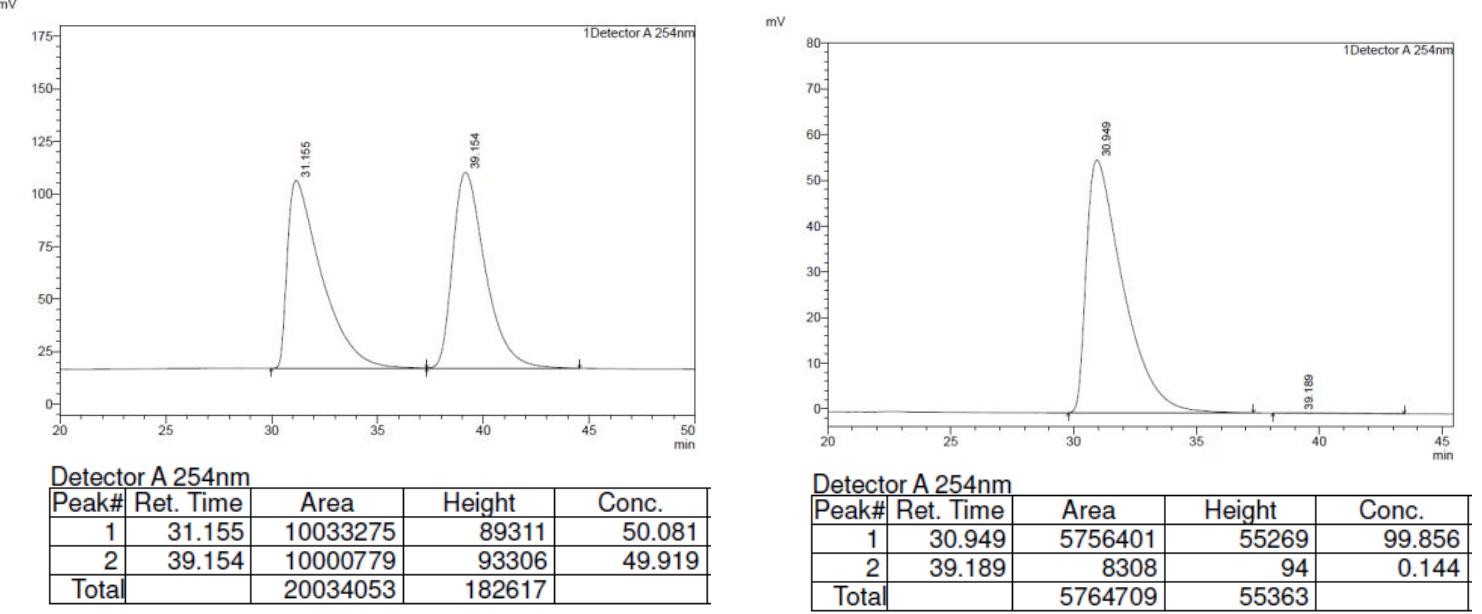

\section{$(R, E)$-6-bromo-4-styryl-3, 4-dihydrobenzo[e][1, 2, 3]oxathiazine 2, 2-dioxide}

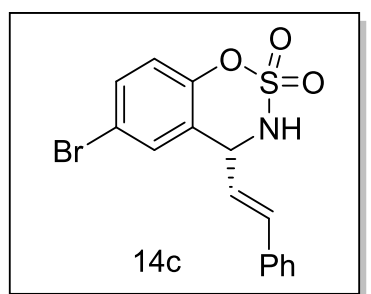

Colorless solid, $52 \%$ Yield.

${ }^{1} \mathbf{H}$ NMR $\left(500 \mathrm{MHz}, \mathrm{CDCl}_{3}\right): \delta$ 7.61-7.35 (m, 8H), $6.97(\mathrm{~d}, \mathrm{~J}=8.8$ $\mathrm{Hz}, 1 \mathrm{H}), 6.91(\mathrm{~d}, \mathrm{~J}=15.6 \mathrm{~Hz}, 1 \mathrm{H}) .6 .29(\mathrm{dd}, J=15.6,8.6 \mathrm{~Hz}, 1 \mathrm{H})$, $5.46(\mathrm{t}, J=8.4 \mathrm{~Hz}, 1 \mathrm{H}), 4.86(\mathrm{~d}, J=8.2 \mathrm{~Hz}, 1 \mathrm{H})$.

${ }^{13} \mathbf{C}$ NMR $\left(125 \mathrm{MHz}, \mathrm{CDCl}_{3}\right): \delta 150.08,137.66,134.90,132.95$, $130.58,129.18,128.90,127.05,123.32,123.14,120.66,118.11$, 59.80 .

HRMS (ESI): m/z Calcd. For $\left[\mathrm{C}_{15} \mathrm{H}_{11} \mathrm{BrNO}_{3} \mathrm{~S}, \mathrm{M}-\mathrm{H}\right]=363.9649$; Found: 363.9650.

Optical Rotation: $[\alpha]_{D}^{22}=-6.60\left(\mathrm{c}=0.50, \mathrm{CH}_{2} \mathrm{Cl}_{2}\right)$. The absolute configuration was assigned according the analogue 14k.

99.6: 0.4 er. HPLC condition: Chiralcel OD-H column, $n$-hexane $/ i-\mathrm{PrOH}=80: 20$, flow rate $=$ $0.8 \mathrm{ml} / \mathrm{min}$, wavelength $=254 \mathrm{~nm}, t_{R}=20.739 \mathrm{~min}$ for major isomer, $t_{R}=27.963 \mathrm{~min}$ for minor isomer. 

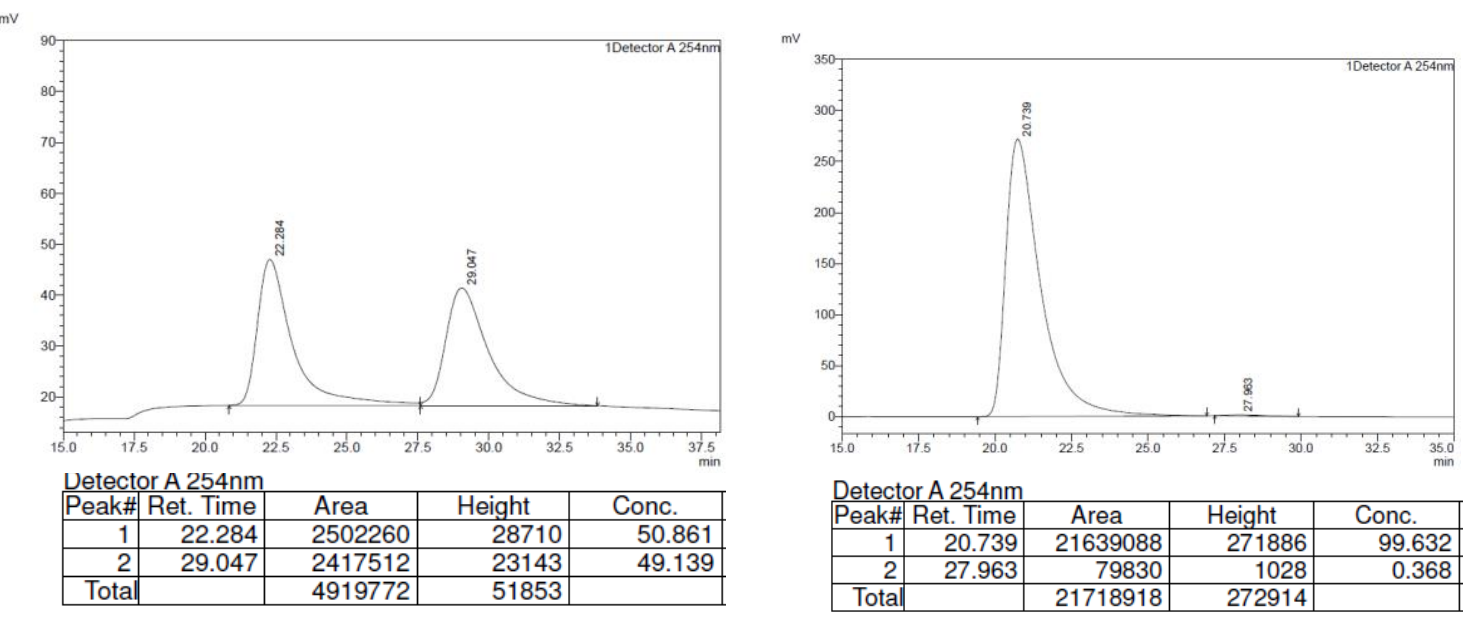

(R, E)-6, 8-dichloro-4-styryl-3, 4-dihydrobenzo[e][1, 2, 3]oxathiazine 2, 2-dioxide

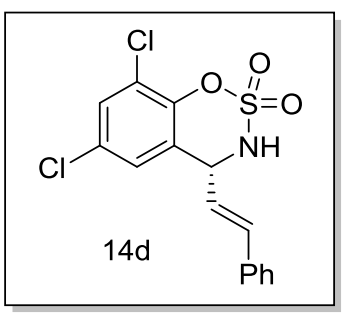

Colorless solid, $69 \%$ Yield.

${ }^{1}$ H NMR $\left(300 \mathrm{MHz}, \mathrm{CDCl}_{3}\right): \delta 7.61-7.33(\mathrm{~m}, 6 \mathrm{H}), 7.15(\mathrm{dd}, \mathrm{J}=2.4$, $1.0 \mathrm{~Hz}, 1 \mathrm{H}), 6.93(\mathrm{~d}, \mathrm{~J}=15.6 \mathrm{~Hz}, 1 \mathrm{H}), 6.28(\mathrm{dd}, \mathrm{J}=15.6,8.7 \mathrm{~Hz}$, $1 \mathrm{H}), 5.54-5.41(\mathrm{~m}, 1 \mathrm{H}), 5.09(\mathrm{~d}, \mathrm{~J}=8.3 \mathrm{~Hz}, 1 \mathrm{H})$.

${ }^{13} \mathrm{C}$ NMR $\left(125 \mathrm{MHz}, \mathrm{CDCl}_{3}\right): \delta 145.74,137.99,134.74,130.38$, $130.34,129.29,128.92,127.06,126.09,124.88,124.32,122.68$, 60.07 .

HRMS (ESI): $\mathrm{m} / \mathrm{z}$ Calcd. for $\left[\mathrm{C}_{15} \mathrm{H}_{10} \mathrm{Cl}_{2} \mathrm{NO}_{3} \mathrm{~S}, \mathrm{M}-\mathrm{H}\right]=353.9764$; Found: 353.9767.

Optical Rotation: $[\alpha]_{D}^{22}=-5.50\left(\mathrm{c}=0.40, \mathrm{CH}_{2} \mathrm{Cl}_{2}\right)$. The absolute configuration was assigned according the analogue $\mathbf{1 4 k}$.

99.5 0.5 er. HPLC condition: Chiralpak IE column, $n$-hexane $/ i$-PrOH $=90: 10$, flow rate $=0.9$ $\mathrm{ml} / \mathrm{min}$, wavelength $=254 \mathrm{~nm}, t_{R}=8.958 \mathrm{~min}$ for major isomer, $t_{R}=11.770 \min$ for minor isomer.
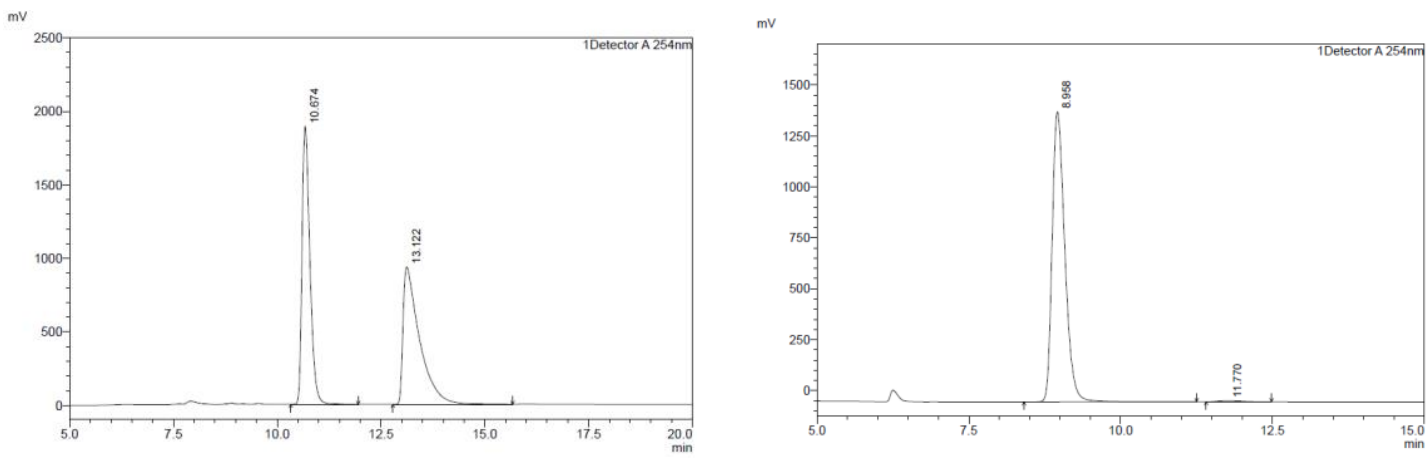

Detector A $254 \mathrm{~nm}$
\begin{tabular}{|r|r|r|r|r|}
\hline Peak\# & Ret. Time & Area & Height & Conc. \\
\hline 1 & 10.674 & 24910638 & 1889787 & 49.159 \\
\hline 2 & 13.122 & 25763221 & 934524 & 50.841 \\
\hline Total & & 50673859 & 2824311 & \\
\hline
\end{tabular}

Detector A $254 \mathrm{~nm}$
\begin{tabular}{|r|r|r|r|r|}
\hline Peak\# & Ret. Time & \multicolumn{1}{c|}{ Area } & Height & \multicolumn{1}{c|}{ Conc. } \\
\hline 1 & 8.958 & 20625749 & 1423136 & 99.436 \\
\hline 2 & 11.770 & 116979 & 4624 & 0.564 \\
\hline Total & & 20742728 & 1427761 & \\
\hline
\end{tabular}




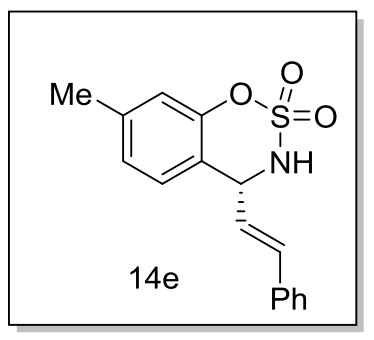

59.87, 20.96.

HRMS (ESI): $\mathrm{m} / \mathrm{z}$ Calcd. For $\left[\mathrm{C}_{16} \mathrm{H}_{15} \mathrm{NNaO}_{3} \mathrm{~S}, \mathrm{M}+\mathrm{Na}\right]^{+}: 324.0665$; Found: 324.0663.

Optical Rotation: $[\alpha]_{D}^{22}=-8.80\left(\mathrm{c}=0.45, \mathrm{CH}_{2} \mathrm{Cl}_{2}\right)$. The absolute configuration was assigned according the analogue $\mathbf{1 4 k}$.

99.8: 0.2 er. HPLC condition: Chiralcel OD-H column, $n$-hexane $/ i-\mathrm{PrOH}=80: 20$, flow rate $=$ $0.8 \mathrm{ml} / \mathrm{min}$, wavelength $=254 \mathrm{~nm}, t_{R}=22.787 \mathrm{~min}$ for major isomer, $t_{R}=28.820 \mathrm{~min}$ for minor isomer.
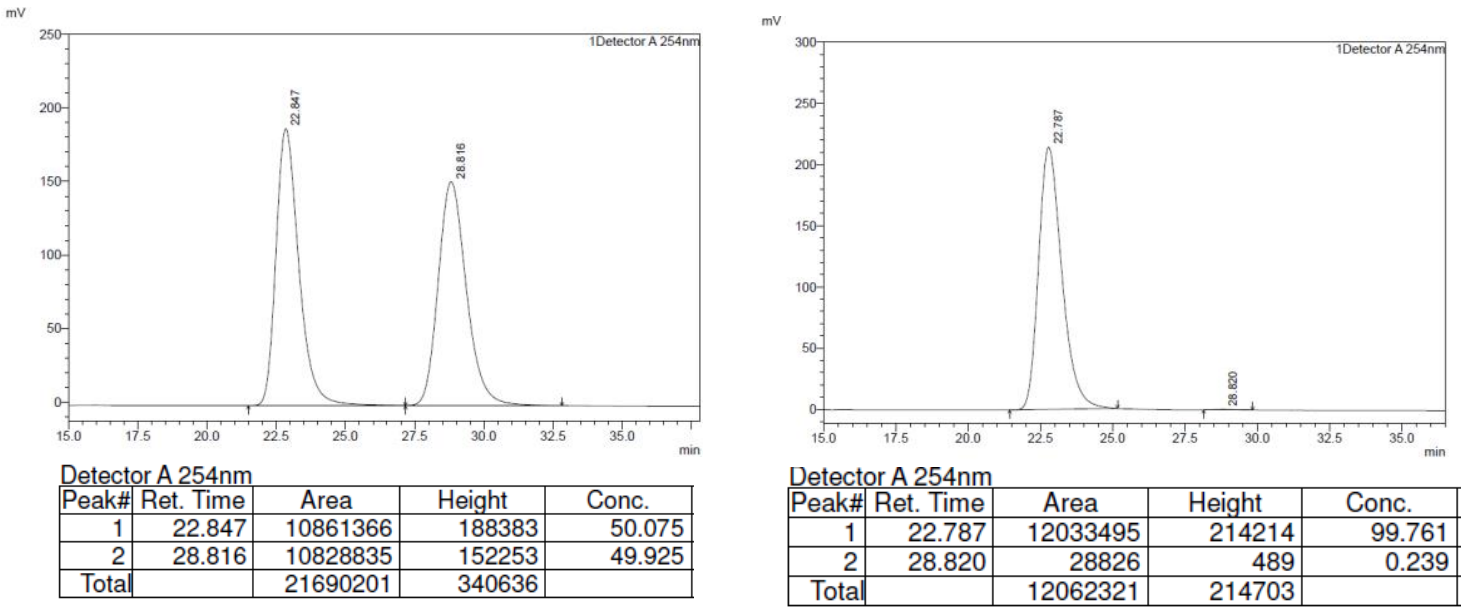

\section{(R, E)-4-(4-methoxystyryl)-3, 4-dihydrobenzo[e][1, 2, 3]oxathiazine 2, 2-dioxide}

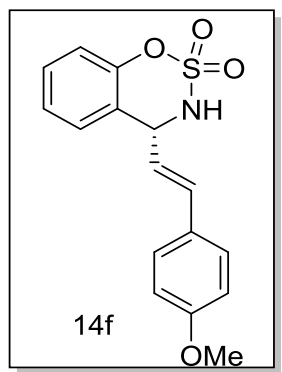

Found: 340.0620 .
Pale yellow solid, $85 \%$ yield.

${ }^{1} \mathbf{H}$ NMR $\left(500 \mathrm{MHz}, \mathrm{CDCl}_{3}\right): \delta 7.45-7.32(\mathrm{~m}, 3 \mathrm{H}), 7.22(\mathrm{~m}, 2 \mathrm{H}), 7.06$ $(\mathrm{d}, \mathrm{J}=8.2 \mathrm{~Hz}, 1 \mathrm{H}), 6.92(\mathrm{~d}, \mathrm{~J}=8.7 \mathrm{~Hz}, 2 \mathrm{H}), 6.83(\mathrm{~d}, \mathrm{~J}=15.6 \mathrm{~Hz}, 1 \mathrm{H})$, $6.15(\mathrm{dd}, \mathrm{J}=15.6,8.6 \mathrm{~Hz}, 1 \mathrm{H}), 5.45(\mathrm{t}, \mathrm{J}=8.5 \mathrm{~Hz}, 1 \mathrm{H}), 4.85(\mathrm{~d}, \mathrm{~J}=8.2$ $\mathrm{Hz}, 1 \mathrm{H}), 3.85$ (s, 3H).

${ }^{13}$ C NMR $\left(125 \mathrm{MHz}, \mathrm{CDCl}_{3}\right): \delta 160.23,150.97,136.44,129.80,128.29$, $127.90,125.34,121.75,121.53,118.84,114.26,60.22,55.38$.

HRMS (ESI): $\mathrm{m} / \mathrm{z}$ Calcd. For $\left[\mathrm{C}_{16} \mathrm{H}_{15} \mathrm{NNaO}_{4} \mathrm{~S}, \mathrm{M}+\mathrm{Na}\right]^{+}: 340.0614$; 
Optical Rotation: $[\alpha]_{D}^{22}=-3.80\left(\mathrm{c}=0.50, \mathrm{CHCl}_{3}\right)$. The absolute configuration was assigned according the analogue 14k.

99.6: 0.4 er. HPLC condition: Chiralcel OD-H column, $n$-hexane $/ i-\mathrm{PrOH}=80: 20$, flow rate $=$ $0.8 \mathrm{ml} / \mathrm{min}$, wavelength $=254 \mathrm{~nm}, \mathrm{t}_{\mathrm{R}}=20.035 \mathrm{~min}$ for major isomer, $\mathrm{t}_{\mathrm{R}}=26.855 \mathrm{~min}$ for minor isomer.
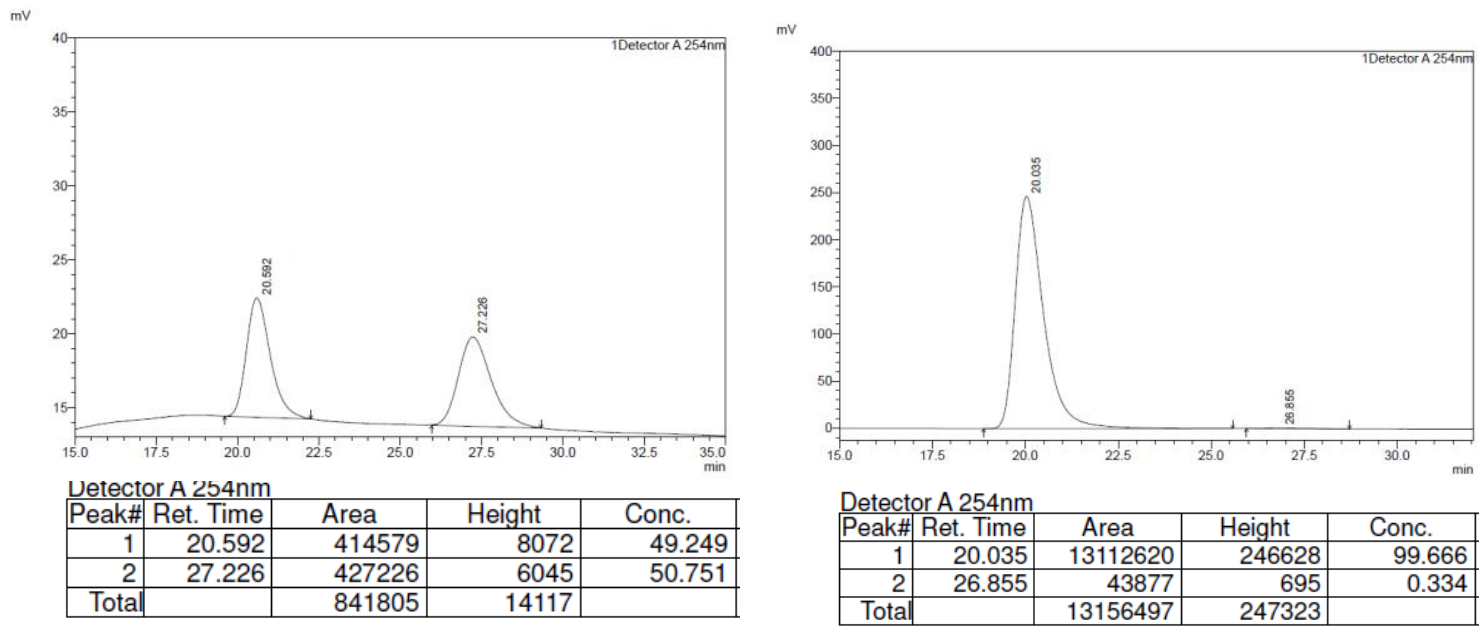

\section{(R, E)-4-(4-methylstyryl)-3, 4-dihydrobenzo[e][1, 2, 3]oxathiazine 2, 2-dioxide}

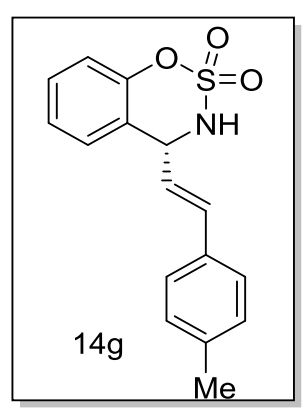

Found: 324.0664.

Optical Rotation: $[\alpha]^{22}=-12.4\left(\mathrm{c}=0.40, \mathrm{CH}_{2} \mathrm{Cl}_{2}\right)$. The absolute configuration was assigned according the analogue $14 \mathbf{k}$.

99.6: 0.4 er. HPLC condition: Chiralcel OD-H column, $n$-hexane $/ i-\mathrm{PrOH}=80: 20$, flow rate $=$ $0.8 \mathrm{ml} / \mathrm{min}$, wavelength $=254 \mathrm{~nm}, t_{R}=14.591 \mathrm{~min}$ for major isomer, $t_{R}=25.394 \mathrm{~min}$ for minor isomer.
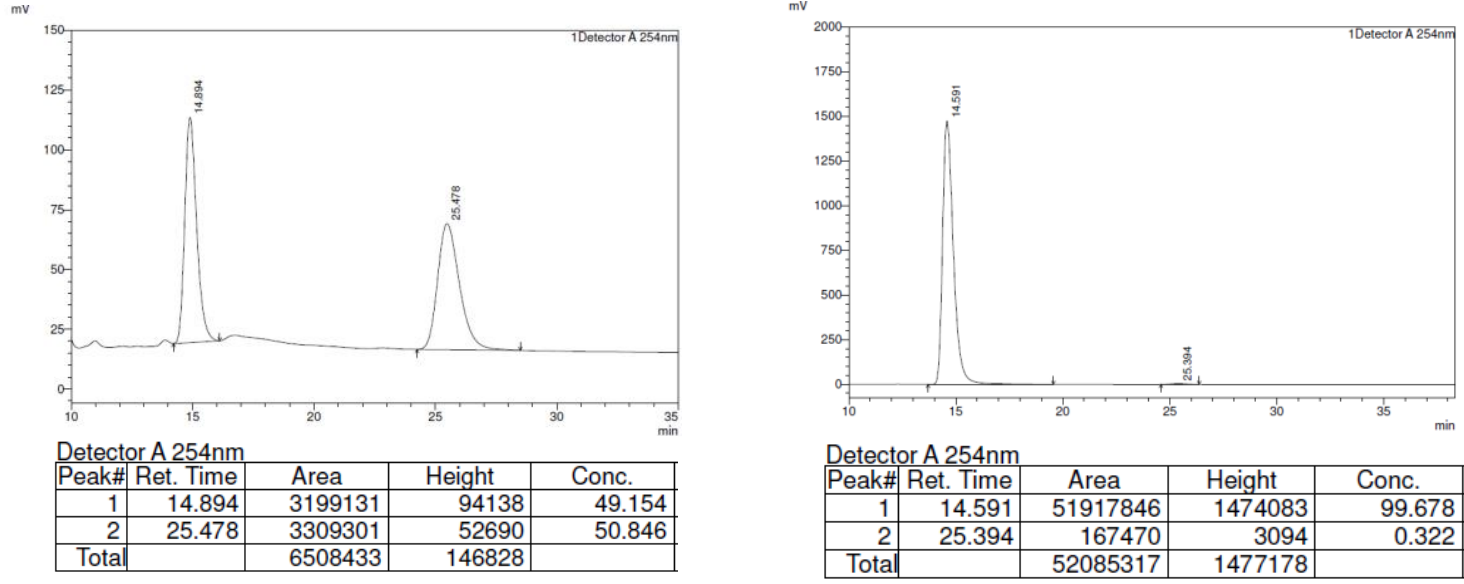


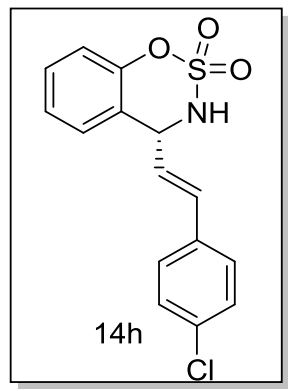

Colorless solid, $77 \%$ yield.

${ }^{1} \mathbf{H}$ NMR $\left(300 \mathrm{MHz}, \mathrm{CDCl}_{3}\right): \delta 7.47-7.32(\mathrm{~m}, 5 \mathrm{H}), 7.23(\mathrm{dd}, \mathrm{J}=3.2$, $2.5 \mathrm{~Hz}, 2 \mathrm{H}), 7.07(\mathrm{~d}, \mathrm{~J}=8.1 \mathrm{~Hz}, 1 \mathrm{H}), 6.84(\mathrm{~d}, \mathrm{~J}=15.7 \mathrm{~Hz}, 1 \mathrm{H}), 6.33(\mathrm{dd}$, $\mathrm{J}=15.7,8.5 \mathrm{~Hz}, 1 \mathrm{H}), 5.48(\mathrm{t}, \mathrm{J}=8.3 \mathrm{~Hz}, 1 \mathrm{H}), 5.01(\mathrm{~d}, \mathrm{~J}=8.2 \mathrm{~Hz}, 1 \mathrm{H})$.

${ }^{13}$ C NMR $\left(75 \mathrm{MHz}, \mathrm{CDCl}_{3}\right): \delta 150.83,135.30,134.59,133.62,129.87$, $128.95,128.08,127.70,125.37,124.73,120.89,118.87,59.83$.

HRMS (ESI): $\mathrm{m} / \mathrm{z}$ Calcd. For $\left[\mathrm{C}_{15} \mathrm{H}_{11} \mathrm{FNO}_{3} \mathrm{~S}, \mathrm{M}-\mathrm{H}\right]: 320.0154$; Found: 320.0159

Optical Rotation: $[\alpha]^{22}=-40.23\left(\mathrm{c}=0.40, \mathrm{CH}_{2} \mathrm{Cl}_{2}\right)$. The absolute configuration was assigned according the analogue 14k.

99.8: 0.2 er. HPLC condition: Chiralcel OD-H column, $n$-hexane $/ i-\mathrm{PrOH}=80: 20$, flow rate $=$ $0.8 \mathrm{ml} / \mathrm{min}$, wavelength $=254 \mathrm{~nm}, \mathrm{t}_{\mathrm{R}}=15.154 \mathrm{~min}$ for major isomer, $\mathrm{t}_{\mathrm{R}}=20.669 \mathrm{~min}$ for minor isomer.
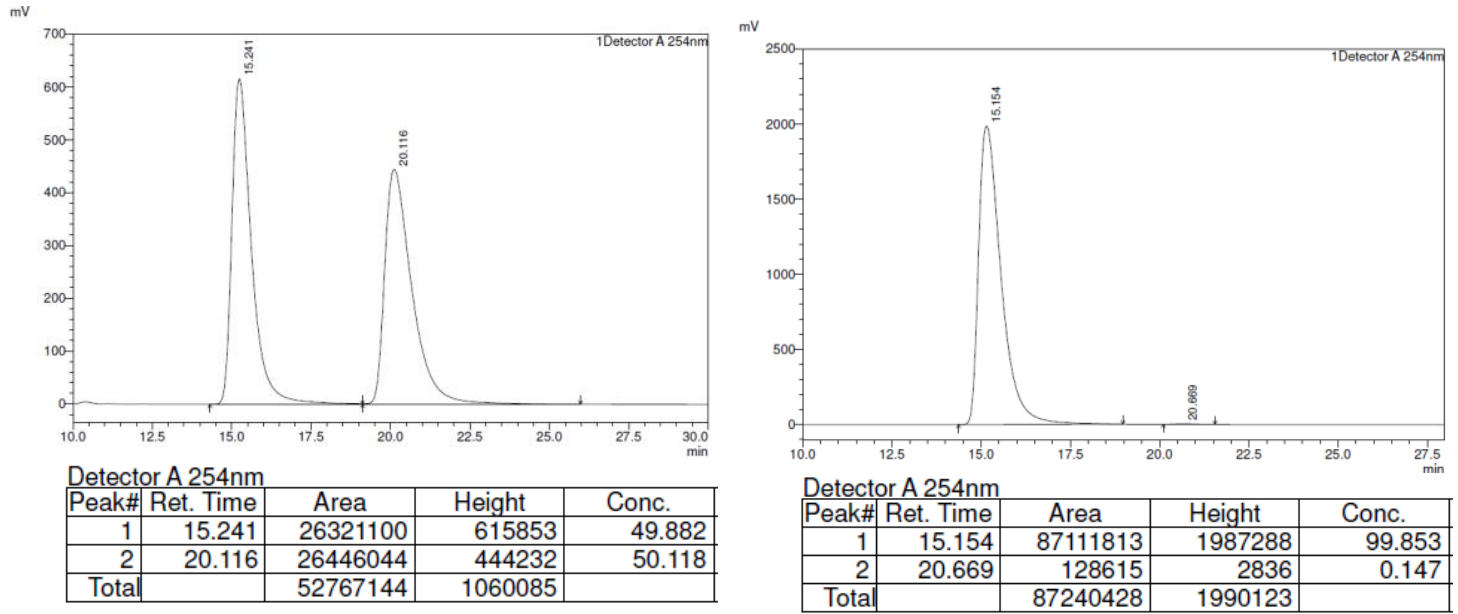

\section{(R, E)-4-(4-fluorostyryl)-3, 4-dihydrobenzo[e][1, 2, 3]oxathiazine 2, 2-dioxide}

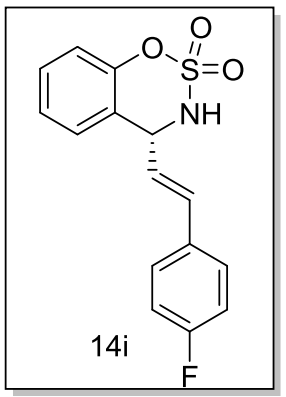

Colorless solid, $58 \%$ yield.

${ }^{1} \mathbf{H}$ NMR $\left(300 \mathrm{MHz}, \mathrm{CDCl}_{3}\right): \delta 7.62-7.34(\mathrm{~m}, 3 \mathrm{H}), 7.30-7.18(\mathrm{~m}, 2 \mathrm{H})$, $7.18-7.02(\mathrm{~m}, 3 \mathrm{H}), 6.86(\mathrm{~d}, \mathrm{~J}=15.7 \mathrm{~Hz}, 1 \mathrm{H}), 6.26(\mathrm{dd}, \mathrm{J}=15.7,8.5 \mathrm{~Hz}$, $1 \mathrm{H}), 5.48(\mathrm{t}, \mathrm{J}=8.4 \mathrm{~Hz}, 1 \mathrm{H}), 4.96(\mathrm{~d}, \mathrm{~J}=8.2 \mathrm{~Hz}, 1 \mathrm{H})$.

${ }^{13}$ C NMR $\left(75 \mathrm{MHz}, \mathrm{CDCl}_{3}\right): \delta 164.61,161.31,150.84,135.45,131.33$, $129.84,128.60,128.50,127.72,125.34,123.84,121.04,118.84,115.92$, $115.63,59.90$.

${ }^{19} \mathbf{F}$ NMR $\left(282 \mathrm{MHz}, \mathrm{CDCl}_{3}\right): \delta-112.09(\mathrm{ddd}, \mathrm{J}=12.4,8.1,4.3 \mathrm{~Hz}, 1 \mathrm{~F})$.

HRMS (ESI): $\mathrm{m} / \mathrm{z}$ Calcd. For $\left[\mathrm{C}_{15} \mathrm{H}_{11} \mathrm{FNO}_{3} \mathrm{~S}, \mathrm{M}-\mathrm{H}\right]=304.0449$; Found: 304.0451

Optical Rotation: $[\alpha]_{D}^{22}=-23.43\left(c=0.30, \mathrm{CH}_{2} \mathrm{Cl}_{2}\right)$. The absolute configuration was assigned according the analogue 14k. 
99:1 er. HPLC condition: Chiralcel OD-H column, $n$-hexane $/ i$ - $\mathrm{PrOH}=80: 20$, flow rate $=0.8$ $\mathrm{ml} / \mathrm{min}$, wavelength $=254 \mathrm{~nm}, \mathrm{t}_{\mathrm{R}}=13.841 \mathrm{~min}$ for major isomer, $\mathrm{t}_{\mathrm{R}}=16.899 \min$ for minor isomer.
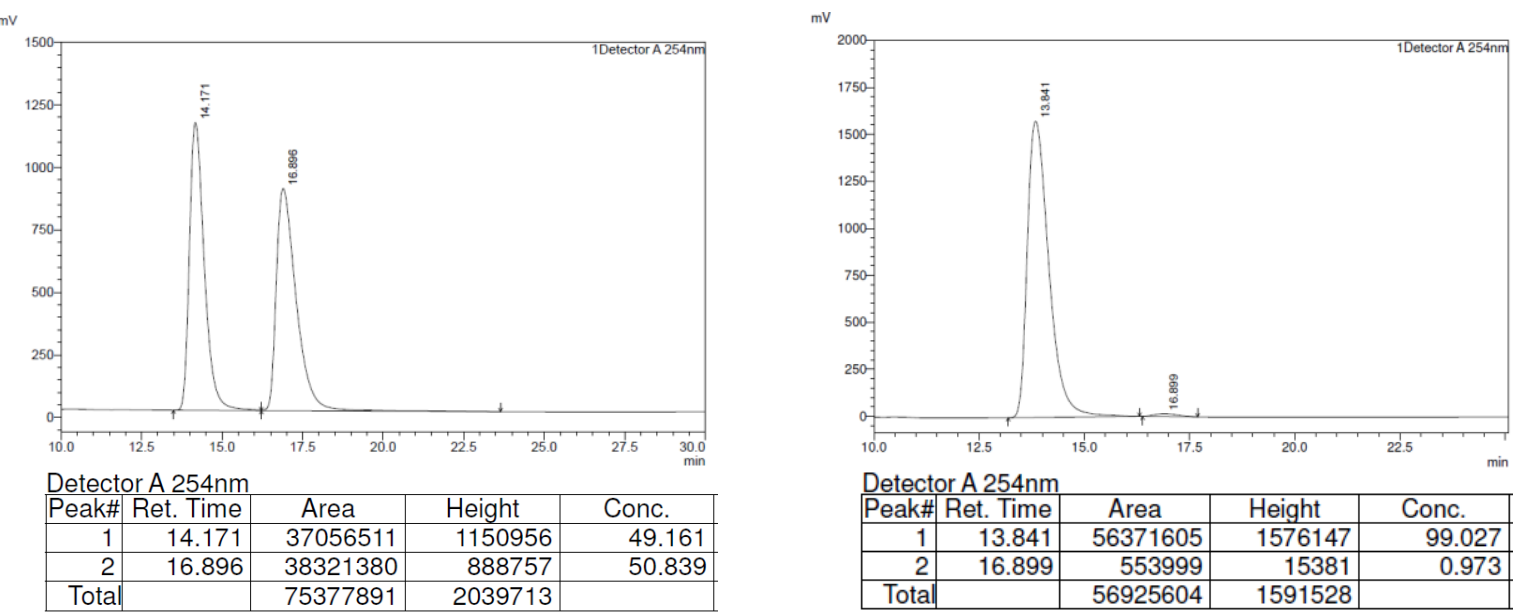

(R, E)-6-bromo-4-(4-methoxystyryl)-3, 4-dihydrobenzo[e][1, 2, 3]oxathiazine 2,

\section{2-dioxide}<smiles>COc1ccc(/C=C/[C@H]2NS(=O)(=O)Oc3ccc(Br)cc32)cc1</smiles>

Found: 393.9752

Pale yellow solid, $78 \%$ yield.

${ }^{1} \mathbf{H}$ NMR $\left(300 \mathrm{MHz}, \mathrm{CDCl}_{3}\right): \delta 7.54-7.34(\mathrm{~m}, 4 \mathrm{H}), 7.02-6.91(\mathrm{~m}$, $3 \mathrm{H}), 6.86(\mathrm{~d}, \mathrm{~J}=15.6 \mathrm{~Hz}, 1 \mathrm{H}), 6.12(\mathrm{dd}, \mathrm{J}=15.6,8.7 \mathrm{~Hz}, 1 \mathrm{H}), 5.43(\mathrm{t}$, $\mathrm{J}=8.4 \mathrm{~Hz}, 1 \mathrm{H}), 4.95(\mathrm{~d}, \mathrm{~J}=8.3 \mathrm{~Hz}, 1 \mathrm{H}), 3.87(\mathrm{~s}, 3 \mathrm{H})$.

${ }^{13} \mathrm{C}$ NMR $\left(125 \mathrm{MHz}, \mathrm{CDCl}_{3}\right): \delta 160.45,150.05,137.22,132.84$, $130.64,128.41,127.62,123.62,120.78,120.58,118.04,114.33$, 59.97, 55.39.

HRMS (ESI): m/z Calcd. For $\left.\left[\mathrm{C}_{16} \mathrm{H}_{13} \mathrm{BrNO}_{4} \mathrm{~S}, \mathrm{M}-\mathrm{H}\right]\right]^{-}: 393.9754$;

Optical Rotation: $[\alpha]_{D}^{22}=-9.03\left(\mathrm{c}=0.4, \mathrm{CH}_{2} \mathrm{Cl}_{2}\right)$. The absolute configuration was assigned according the analogue $\mathbf{1 4 k}$.

99.6: 0.4 er. HPLC condition: Chiralcel OD-H column, $n$-hexane $/ i-\mathrm{PrOH}=80 / 20$, flow rate $=$ $0.8 \mathrm{ml} / \mathrm{min}$, wavelength $=254 \mathrm{~nm}, t_{R}=20.739 \mathrm{~min}$ for major isomer, $t_{R}=27.963 \mathrm{~min}$ for minor isomer.
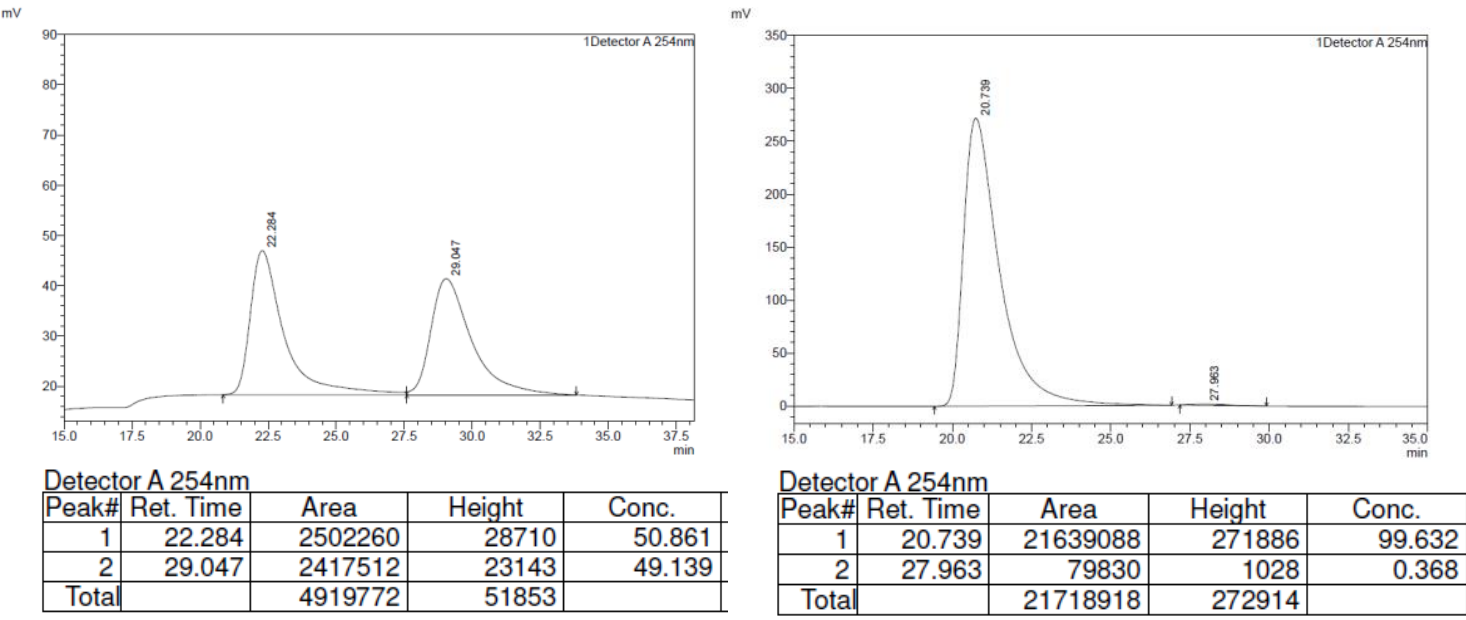


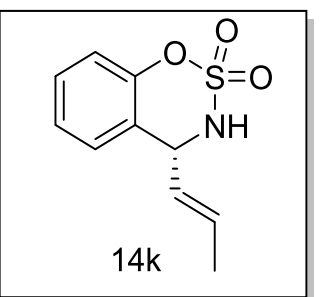

Colorless solid, $65 \%$ yield.

${ }^{1} \mathbf{H}$ NMR $\left(300 \mathrm{MHz}, \mathrm{CDCl}_{3}\right): \delta 7.41-7.32(\mathrm{~m}, 1 \mathrm{H}), 7.21(\mathrm{dd}, \mathrm{J}=3.4$, $2.2 \mathrm{~Hz}, 2 \mathrm{H}), 7.04(\mathrm{~d}, \mathrm{~J}=8.1 \mathrm{~Hz}, 1 \mathrm{H}), 6.14-5.98(\mathrm{~m}, 1 \mathrm{H}), 5.73-$ $5.55(\mathrm{~m}, 1 \mathrm{H}), 5.29(\mathrm{t}, \mathrm{J}=8.5 \mathrm{~Hz}, 1 \mathrm{H}), 4.67(\mathrm{~d}, \mathrm{~J}=8.3 \mathrm{~Hz}, 1 \mathrm{H}), 1.88$ (dd, $\mathrm{J}=6.6,1.6 \mathrm{~Hz}, 3 \mathrm{H})$.

${ }^{13} \mathrm{C}$ NMR $\left(75 \mathrm{MHz}, \mathrm{CDCl}_{3}\right): \delta 150.84,134.07,129.58,127.68$, $126.51,125.13,121.51,118.65,59.82,17.74$.

HRMS (EI): $\mathrm{m} / \mathrm{z}$ Calcd. for $\mathrm{C}_{10} \mathrm{H}_{10} \mathrm{NO}_{3} \mathrm{~S}$ [M-H] $: 224.0387$, found: 224.0381

Optical Rotation: $[\alpha]_{D}^{22}=-18.9\left(\mathrm{c}=0.37, \mathrm{CH}_{2} \mathrm{Cl}_{2}\right)$. The absolute configuration was assigned according x-ray crystallography..

99.8: 0.2 er. HPLC condition: Chiralcel OD-H column, $n$-hexane $/ i-\mathrm{PrOH}=95 / 5$, flow rate $=$ $1.0 \mathrm{ml} / \mathrm{min}$, wavelength $=254 \mathrm{~nm}, \mathrm{t}_{\mathrm{R}}=15.761 \mathrm{~min}$ for minor isomer, $\mathrm{t}_{\mathrm{R}}=21.047 \mathrm{~min}$ for major isomer.
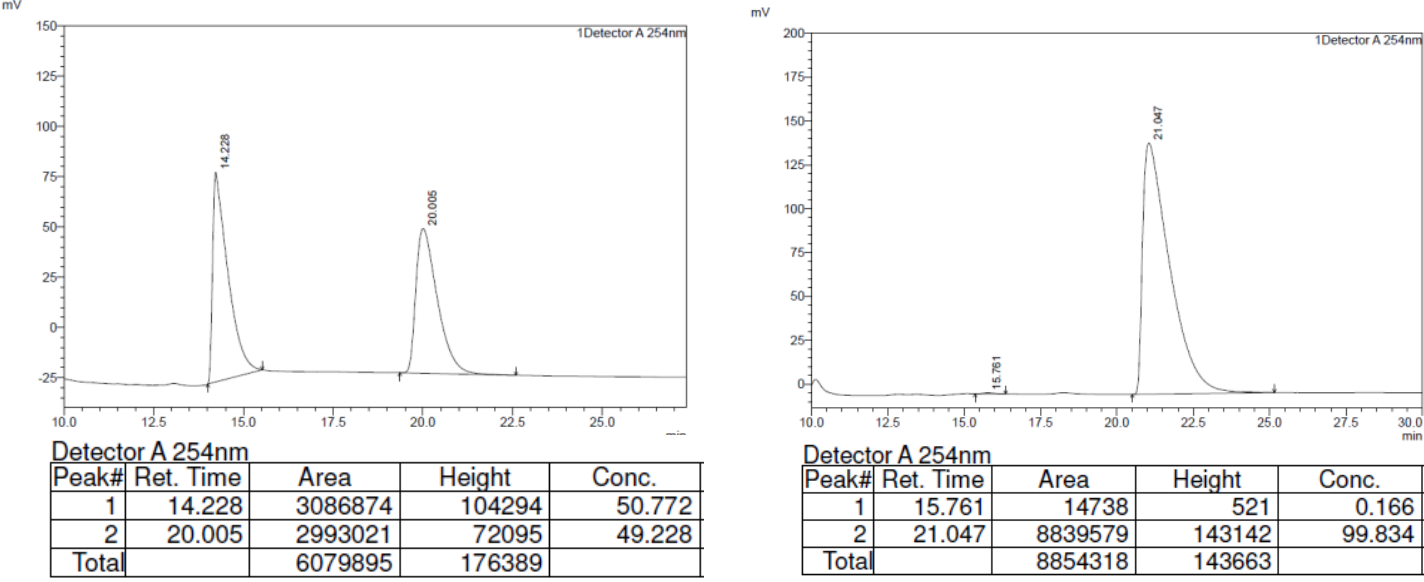

\section{(R, E)-7-methyl-4-(pent-1-en-1-yl)-3, 4-dihydrobenzo[e][1, 2, 3]oxathiazine 2,}

\section{2-dioxide}

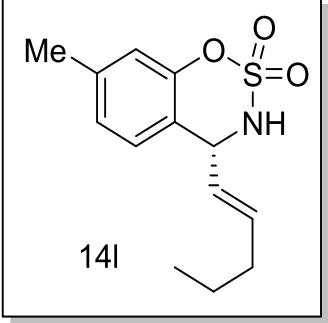

Colorless solid, $56 \%$ yield.

${ }^{1} \mathbf{H}$ NMR $\left(300 \mathrm{MHz}, \mathrm{CDCl}_{3}\right): \delta 7.14-6.93(\mathrm{~m}, 2 \mathrm{H}), 6.86(\mathrm{~s}, 1 \mathrm{H})$, $6.13-5.95(\mathrm{~m}, 1 \mathrm{H}), 5.59(\mathrm{ddt}, \mathrm{J}=15.1,8.4,1.4 \mathrm{~Hz}, 1 \mathrm{H}), 5.25(\mathrm{t}, \mathrm{J}=$ $8.4 \mathrm{~Hz}, 1 \mathrm{H}), 4.60(\mathrm{~d}, \mathrm{~J}=8.4 \mathrm{~Hz}, 1 \mathrm{H}), 2.38(\mathrm{~s}, 3 \mathrm{H}), 2.22-2.10(\mathrm{~m}$, $2 \mathrm{H}), 1.49(\mathrm{~m}, 2 \mathrm{H}), 0.99(\mathrm{t}, \mathrm{J}=7.4 \mathrm{~Hz}, 3 \mathrm{H})$.

${ }^{13} \mathrm{C}$ NMR (75 MHz, $\left.\mathrm{CDCl}_{3}\right): \delta 150.66,140.12,138.88,127.35$, $126.02,125.58,118.87,118.45,59.71,34.14,21.87,20.88,13.57$.

HRMS (ESI): $\mathrm{m} / \mathrm{z}$ Calcd. For $\left[\mathrm{C}_{13} \mathrm{H}_{17} \mathrm{NNaO}_{3} \mathrm{~S}, \mathrm{M}+\mathrm{Na}\right]^{+}: 290.0821$; Found: 290.0826

Optical Rotation: $[\alpha]_{D}^{22}=-14.56\left(\mathrm{c}=0.3, \mathrm{CH}_{2} \mathrm{Cl}_{2}\right)$. The absolute configuration was assigned according the analogue $\mathbf{1 4 k}$.

99.8: 0.2 er. HPLC condition: Chiralcel OD-H column, $n$-hexane $/ i-\mathrm{PrOH}=95: 5$, flow rate $=$ $1 \mathrm{ml} / \mathrm{min}$, wavelength $=254 \mathrm{~nm}, t_{R}=11.458 \mathrm{~min}$ for minor isomer, $t_{R}=15.033$ min for major isomer. 

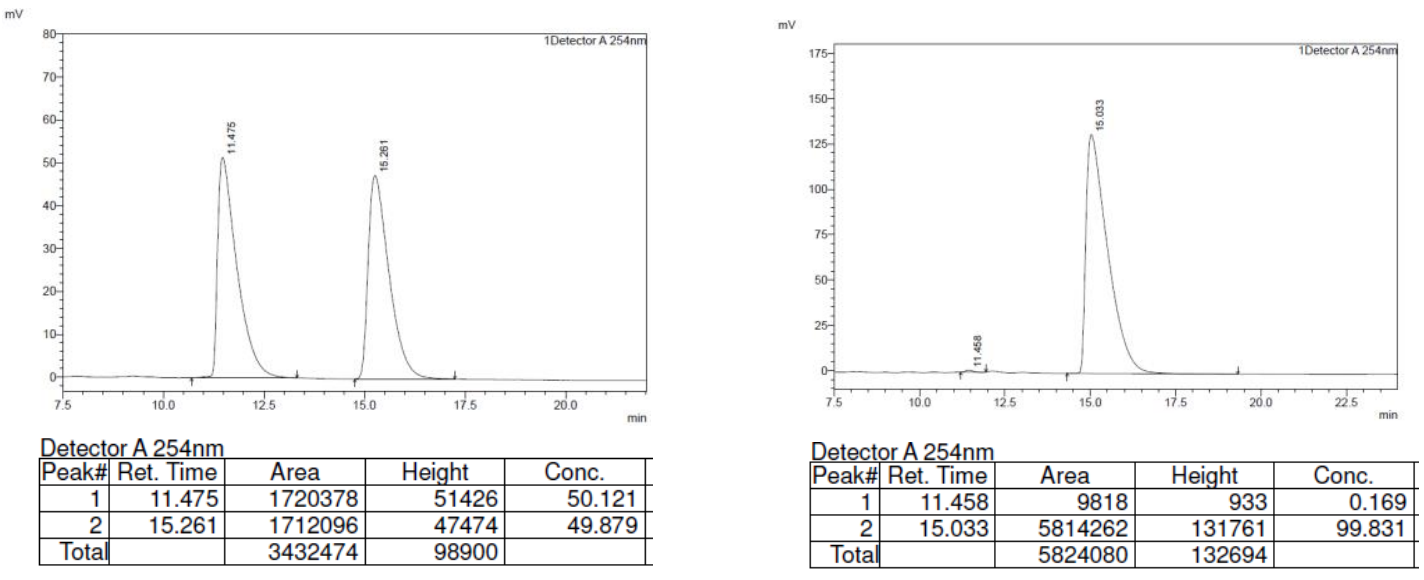

(R)-4-(2-methylprop-1-en-1-yl)-3,4-dihydrobenzo[e][1,2,3]oxathiazine 2,2-dioxide

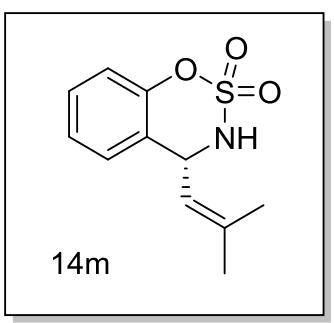

Colorless solid, $70 \%$ yield.

${ }^{1} \mathbf{H}$ NMR $\left(500 \mathrm{MHz}, \mathrm{CDCl}_{3}\right): \delta 7.33(\mathrm{t}, J=7.6 \mathrm{~Hz}, 1 \mathrm{H}), 7.19(\mathrm{t}, J=$ $7.5 \mathrm{~Hz}, 1 \mathrm{H}), 7.13(\mathrm{~d}, J=7.6 \mathrm{~Hz}, 1 \mathrm{H}), 7.01(\mathrm{~d}, J=8.2 \mathrm{~Hz}, 1 \mathrm{H}), 5.58(\mathrm{t}$, $J=8.9 \mathrm{~Hz}, 1 \mathrm{H}), 5.32(\mathrm{dd}, J=8.8,0.9 \mathrm{~Hz}, 1 \mathrm{H}), 4.52(\mathrm{~d}, J=8.8 \mathrm{~Hz}$, $1 \mathrm{H})$.

${ }^{13}$ C NMR $\left(125 \mathrm{MHz}, \mathrm{CDCl}_{3}\right): \delta 151.10,141.81,129.47,127.37$, $125.27,122.47,120.53,118.59,55.40,25.72,18.60$.

HRMS (ESI): $\mathrm{m} / \mathrm{z}$ Calcd. For $\left[\mathrm{C}_{11} \mathrm{H}_{13} \mathrm{NO}_{3} \mathrm{~S}, \mathrm{M}-\mathrm{H}\right]=$ : 238.0543; Found: 238.0538.

Optical Rotation: $[\alpha]^{22}{ }_{D}=-9.0\left(\mathrm{c}=0.3, \mathrm{CH}_{2} \mathrm{Cl}_{2}\right)$. The absolute configuration was assigned according the analogue $\mathbf{1 4 k}$.

99.7: 0.3 er. HPLC condition: Chiralpak IE column, $n$-hexane $/ i$-PrOH $=97: 3$, flow rate $=1$ $\mathrm{ml} / \mathrm{min}$, wavelength $=220 \mathrm{~nm}, t_{R}=24.249 \min$ for major isomer, $t_{R}=32.093 \mathrm{~min}$ for minor isomer.
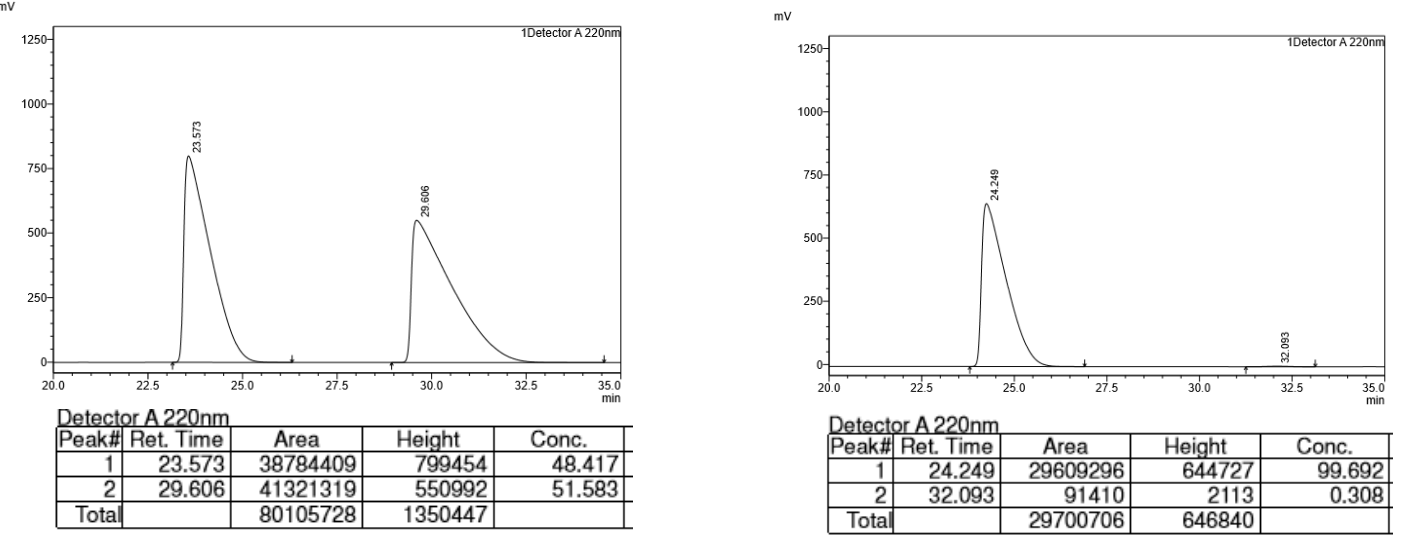


\section{General procedure of the derivatization and analytical data}<smiles>CCOC(=O)C(C)(O)C=Cc1ccccc1</smiles>

2a

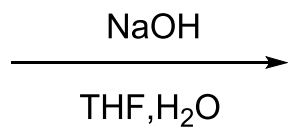

$23^{\circ} \mathrm{C}, 16 \mathrm{~h}$<smiles>CC(O)(C=Cc1ccccc1)C(=O)O</smiles>

3

96: 4 er

To the solution of $2 \mathbf{a}$ (1 equiv.) in THF: $\mathrm{H}_{2} \mathrm{O}$ (1:1) was added 4 equiv. $\mathrm{NaOH}$. The reaction mixture was allowed to stir for $16 \mathrm{~h}$, and then treated with $\mathrm{H}_{2} \mathrm{SO}_{4}$ solution (4 equiv), extracted with ethyl acetate and dried over anhydrous $\mathrm{NaSO}_{4}$. The crude product was recrystallized in ethyl acetate to afford the white crystal in $78 \%$ yield.

${ }^{1}$ H NMR (500 MHz, DMSO): $\delta 7.43(\mathrm{~d}, J=7.4 \mathrm{~Hz}, 2 \mathrm{H}), 7.34(\mathrm{t}, J=7.6 \mathrm{~Hz}, 2 \mathrm{H}), 7.25$ (d, $J=$ $7.3 \mathrm{~Hz}, 1 \mathrm{H}), 6.68(\mathrm{~d}, J=16.0 \mathrm{~Hz}, 1 \mathrm{H}), 6.46(\mathrm{~d}, J=16.0 \mathrm{~Hz}, 1 \mathrm{H}), 1.46(\mathrm{~s}, 3 \mathrm{H})$.

${ }^{13}$ C NMR (125 MHz, DMSO): $\delta$ 176.33, 136.84, 133.61, 129.11, 128.20, 127.99, 126.84, $74.25,27.04$.

HRMS (ESI): m/z Calcd. for $\left[\mathrm{C}_{11} \mathrm{H}_{11} \mathrm{O}_{3}, \mathrm{M}-\mathrm{H}\right]:$ : 191.0708; Found: 191.0712.

Optical Rotation: $[\alpha]^{24}=-60.7\left(\mathrm{c}=0.17, \mathrm{CHCl}_{3}\right)$. The absolute configuration of $\mathbf{3}$ was determined by x-ray crystallography.<smiles>CC(O)(C=Cc1ccccc1)C(=O)O</smiles>

3

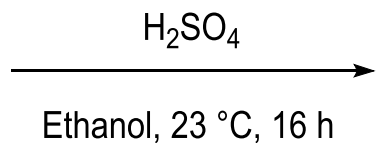

Ethanol, $23^{\circ} \mathrm{C}, 16 \mathrm{~h}$

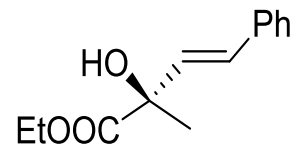

$2 a$

$98: 2$ er

Acid $\mathbf{3}$ was converted back to the ester $\mathbf{2 a}$ to determine its er after recrystallization.

To the solution of 3 ( 1 equiv.) in ethanol was added 1.2 equiv. of $\mathrm{H}_{2} \mathrm{SO}_{4}$. The reaction mixture was allowed to stir for $16 \mathrm{~h}$ at room temperature. The reaction mixture was then washed with saturated $\mathrm{NaHCO}_{3}$ solution, extracted with ethyl acetate and dried over anhydrous $\mathrm{NaSO}_{4}$. The pure product $\mathbf{2 a}$ was isolated in $86 \%$ yield by silica chromatography with ethyl acetate: hexane (1:10). The er was determined to be 98:2.
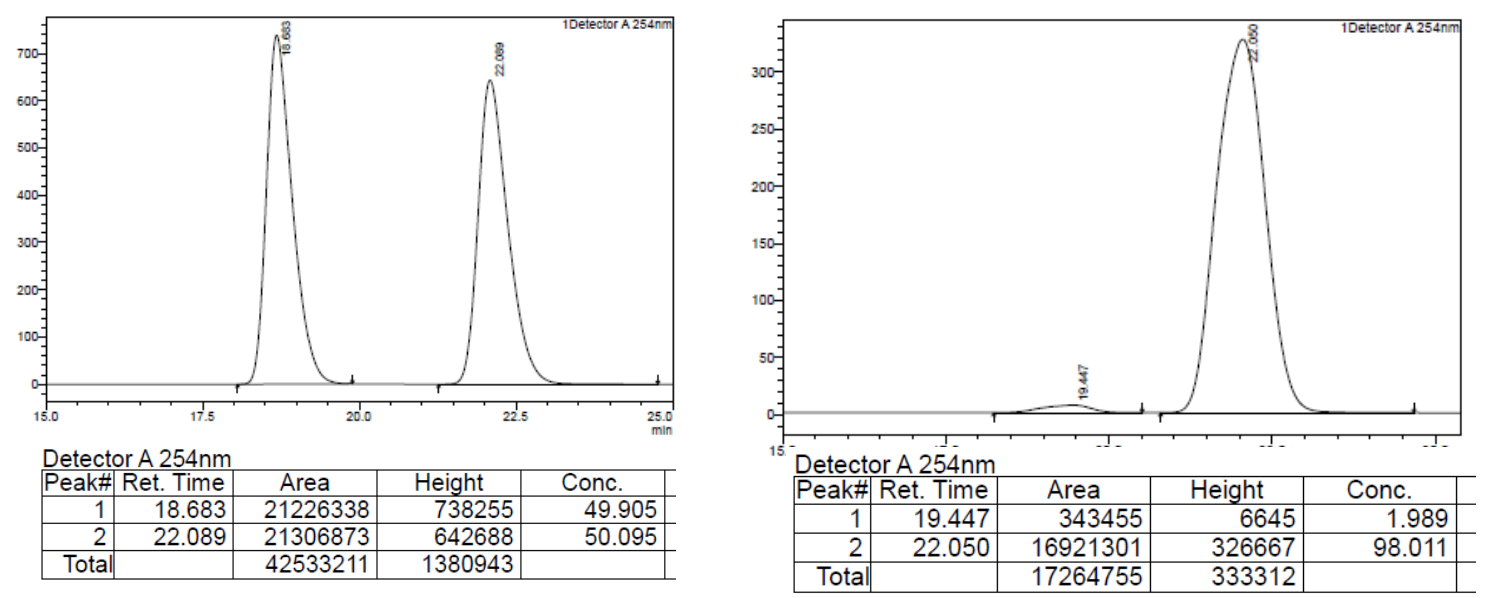
<smiles>CCOC(=O)[C@](C)(O)/C=C/c1ccccc1</smiles>

$2 a$

96:4 er

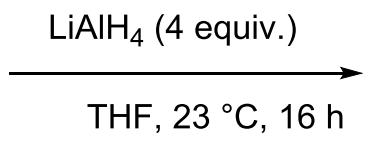

THF, $23^{\circ} \mathrm{C}, 16 \mathrm{~h}$<smiles>C[C@@](O)(/C=C/Pc1ccccc1)CO</smiles>

4

$70 \%, 96: 4$ er

To the solution of 2a ( 1 equiv.) in THF was added 4 equiv. $\mathrm{LiAlH}_{4}$. The reaction mixture was allowed to stir for $16 \mathrm{~h}$ at room temperature under inert atmosphere. The reaction mixture was then quenched with water and washed by $\mathrm{NaOH}$ solution, extracted with ethyl acetate and dried over anhydrous NaSO4. The pure product 4 was isolated in $70 \%$ yield by silica chromatography with ethyl acetate: hexane (1:3).

${ }^{1} \mathbf{H}$ NMR (500 MHz, $\mathrm{CDCl}_{3}$ ): $\delta 7.39(\mathrm{~d}, J=7.4 \mathrm{~Hz}, 2 \mathrm{H}), 7.32(\mathrm{t}, J=7.6 \mathrm{~Hz}, 2 \mathrm{H}), 7.26-7.22$ $(\mathrm{m}, 1 \mathrm{H}), 6.71(\mathrm{~d}, J=16.1 \mathrm{~Hz}, 1 \mathrm{H}), 6.25(\mathrm{~d}, J=16.1 \mathrm{~Hz}, 1 \mathrm{H}), 3.57(\mathrm{dd}, J=41.7,11.0 \mathrm{~Hz}, 2 \mathrm{H})$, $1.39(\mathrm{~s}, 3 \mathrm{H})$.

${ }^{13}$ C NMR (125 MHz, $\left.\mathrm{CDCl}_{3}\right): \delta 136.54,133.12,129.59,128.60,127.72,126.50,73.50,70.03$, 24.44 .

HRMS (ESI): $\mathrm{m} / \mathrm{z}$ Calcd. for $\left[\mathrm{C}_{11} \mathrm{H}_{14} \mathrm{NaO}_{2}, \mathrm{M}+\mathrm{Na}\right]^{+}:$201.0891; Found: 201.0887.

Optical Rotation: $[\alpha]_{\mathrm{D}}^{24}=-25.67\left(\mathrm{c}=0.16, \mathrm{CHCl}_{3}\right)$. The absolute configuration was assigned based on that of $\mathbf{3}$.

95.5:4.5 er. HPLC condition: Chiralpak IE, $n$-hexane $/ i-\mathrm{PrOH}=90: 10$, flow rate $=1 \mathrm{ml} / \mathrm{min}$, wavelength $=254 \mathrm{~nm}, \mathrm{t}_{\mathrm{R}}=10.346 \mathrm{~min}$ for minor isomer, $\mathrm{t}_{\mathrm{R}}=11.341 \mathrm{~min}$ for major isomer.
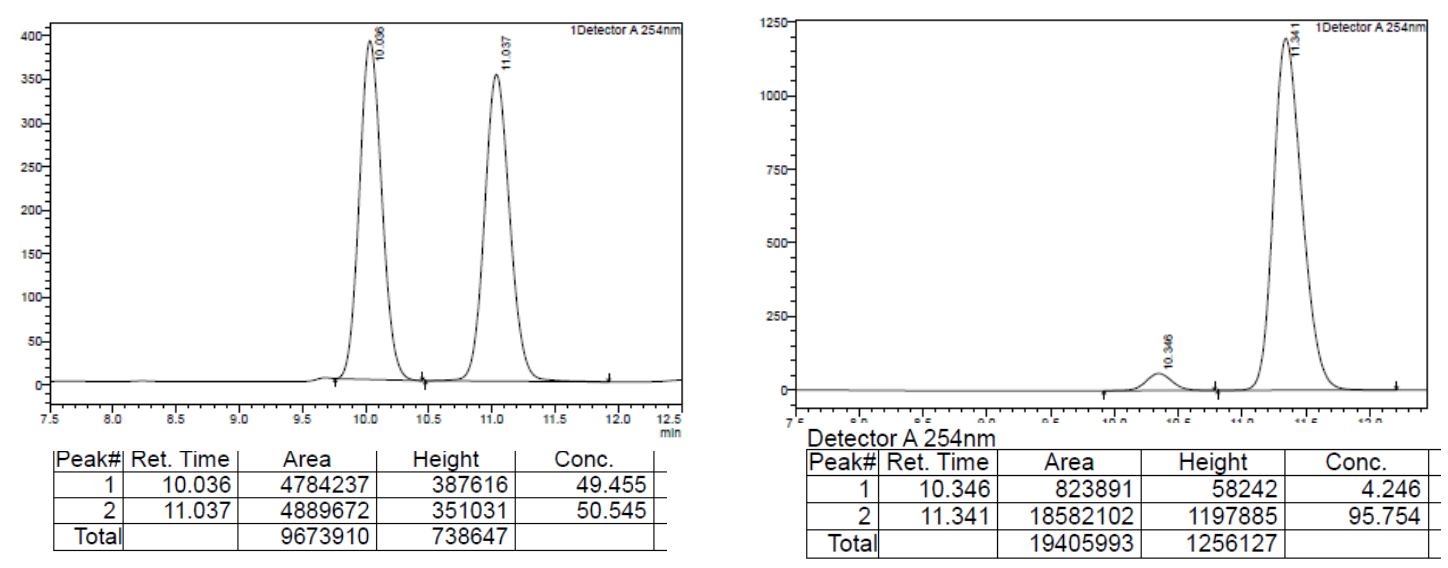


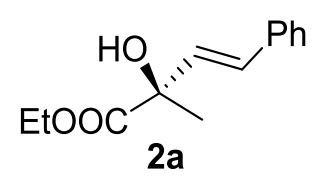

$96: 4$ er

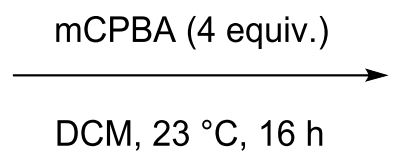

$\mathrm{DCM}, 23^{\circ} \mathrm{C}, 16 \mathrm{~h}$

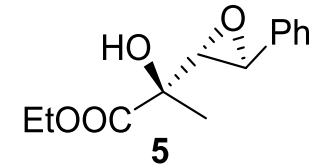

$80 \%, 95.5: 4.5 \mathrm{er}, \mathrm{dr}>20: 1$

To the solution of 2a ( 1 equiv.) in dichloromethane was added 4 equiv. $m$-CPBA. The reaction mixture was allowed to stir for $16 \mathrm{~h}$ at room temperature. The reaction mixture then washed with saturated $\mathrm{NaHCO}_{3}$ solution, extracted with ethyl acetate and dried over anhydrous $\mathrm{NaSO}_{4}$. The pure product $\mathbf{5}$ was isolated by silica chromatography with ethyl acetate: hexane (1:8) as a colorless oil in $80 \%$ yield.

${ }^{1} \mathbf{H}$ NMR $\left(500 \mathrm{MHz}, \mathrm{CDCl}_{3}\right): \delta 7.42-7.29(\mathrm{~m}, 5 \mathrm{H}), 4.41-4.30(\mathrm{~m}, 2 \mathrm{H}), 4.07(\mathrm{~d}, J=1.9 \mathrm{~Hz}$, $1 \mathrm{H}), 3.35(\mathrm{~d}, J=2.0 \mathrm{~Hz}, 1 \mathrm{H}), 3.25(\mathrm{~s}, 1 \mathrm{H}), 1.55(\mathrm{~s}, 3 \mathrm{H}), 1.38(\mathrm{t}, J=7.1 \mathrm{~Hz}, 3 \mathrm{H})$.

${ }^{13} \mathrm{C}$ NMR $\left(126 \mathrm{MHz}, \mathrm{CDCl}_{3}\right): \delta 174.27,136.45,128.53,128.40,125.83,72.02,65.40,62.43$, $54.54,21.88,14.16$.

HRMS (ESI): $\mathrm{m} / \mathrm{z}$ Calcd. for $\left[\mathrm{C}_{13} \mathrm{H}_{16} \mathrm{NaO}_{4}, \mathrm{M}+\mathrm{Na}\right]^{+}: 259.0946$; Found: 259.0942.

Optical Rotation: $[\alpha]^{24}=-15.17\left(\mathrm{c}=0.12, \mathrm{CHCl}_{3}\right)$. The relative configuration was determined by 2-D NMR.

$>$ 20:1 dr, 95.5:4.5 er. HPLC condition: Chiralcel OJ-H, $n$-hexane/ $i-\mathrm{PrOH}=99.5: 0.5$, flow rate $=1 \mathrm{ml} / \mathrm{min}$, wavelength $=227 \mathrm{~nm}, t_{R}=31.742 \mathrm{~min}$ for minor isomer, $t_{R}=35.759 \mathrm{~min}$ for major isomer.
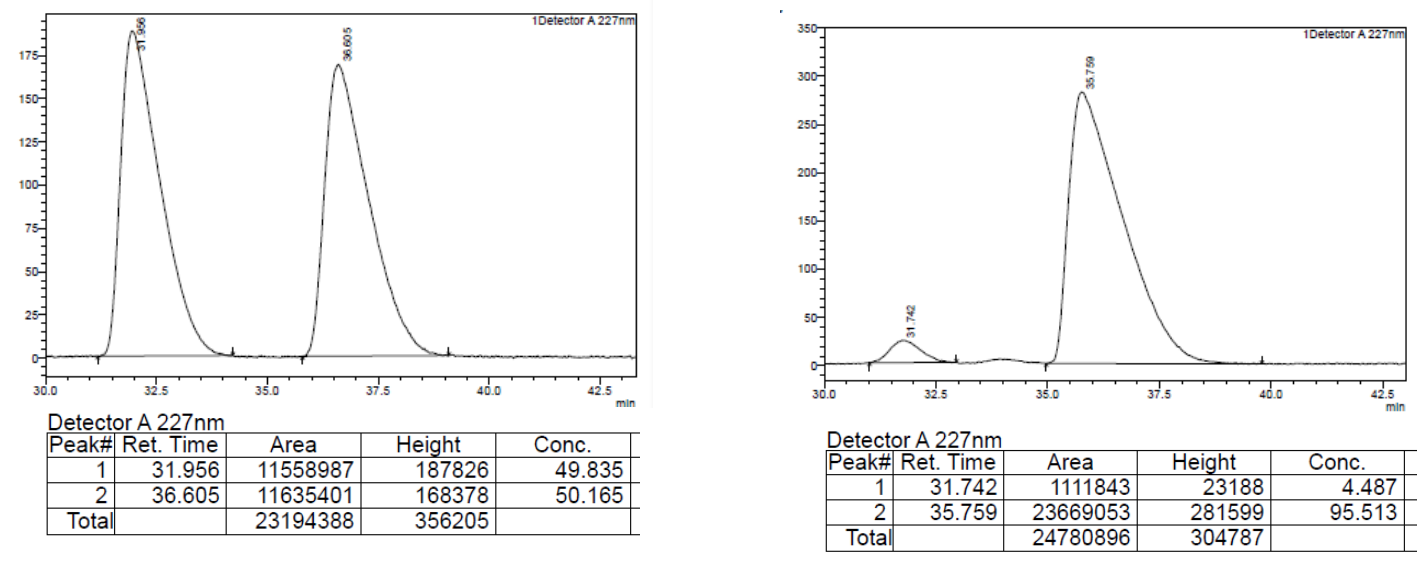


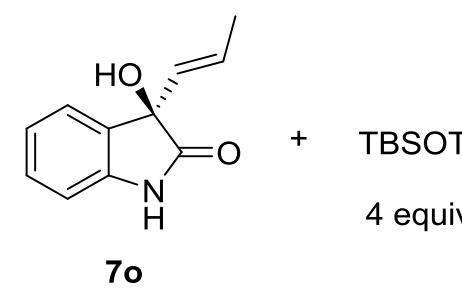

$\mathrm{PdCl}_{2}$ (20 mol\%), CuCl (1equiv.), $\mathrm{O}_{2}$

Dioxan- $\mathrm{H}_{2} \mathrm{O}(7: 1), 70^{\circ} \mathrm{C}, 24 \mathrm{~h}$

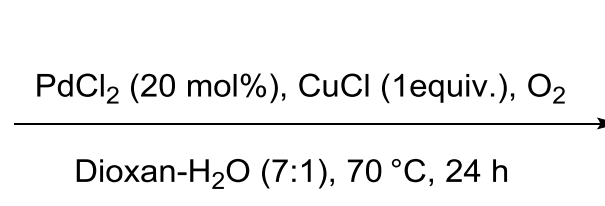

4 equiv. 2.6-Lutidine $\mathrm{DCM}, 23^{\circ} \mathrm{C}, 1 \mathrm{~h} \longrightarrow$

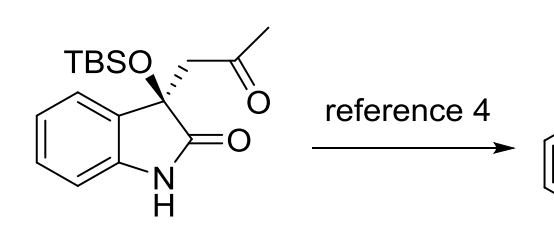
$\begin{array}{lll}9.50 \% & 10\end{array}$<smiles>CC=C[C@]1(OC(F)(F)F)C(=O)Nc2ccccc21</smiles>

$8,72 \%$

The solution of $7 \mathbf{0}(57 \mathrm{mg}, 0.30 \mathrm{mmol}), 2,6$-lutidine $(128 \mathrm{mg}, 1.20 \mathrm{mmol})$ and tert-butyldimethylsilyl triflate $(317 \mathrm{mg}, 1.20 \mathrm{mmol})$ in dry DCM $(4 \mathrm{~mL})$ was allowed to stir at $0{ }^{\circ} \mathrm{C}$ and warm up to room temperature for $1 \mathrm{~h}$. The reaction mixture was diluted with DCM. The organic layer was washed with brine, dried over $\mathrm{NaSO}_{4}$, and evaporated to give a residue, of which a solution in THF-AcOH- $\mathrm{H}_{2} \mathrm{O}(1: 1: 1,1 \mathrm{~mL})$ was stirred at $80{ }^{\circ} \mathrm{C}$ for $3 \mathrm{~h}$. The resulting mixture was diluted with DCM. The organic layer was washed with brine, dried over $\mathrm{NaSO}_{4}$, and concentrated under reduced pressure to give a residue, which was purified by silica gel column chromatography with EtOAc-hexane (1:3) as an eluent to give TBDMS ether $8(65 \mathrm{mg}, 72 \%)$.

A suspension of palladium (II) chloride ( $7 \mathrm{mg}, 0.04 \mathrm{mmol}$ ) and copper (I) chloride (20 $\mathrm{mg}, 0.20 \mathrm{mmol})$ in dioxane- $\mathrm{H}_{2} \mathrm{O}(7: 1,3 \mathrm{~mL})$ was vigorously stirred with bubbling oxygen gas at room temperature for $1 \mathrm{~h}$. Indolin-2-one $8(60 \mathrm{mg}, 0.20 \mathrm{mmol})$ was then added to the mixture. After heating at $70{ }^{\circ} \mathrm{C}$ for $24 \mathrm{~h}$, the reaction mixture was cooled to room temperature and diluted with DCM. The organic layer was washed with brine, dried over $\mathrm{NaSO}_{4}$, and concentrated under reduced pressure. The obtained residue was purified by flash silica gel column chromatography with EtOAc-hexane (2:3) as an eluent to give 9 (31 mg, 50\%) as a yellow solid.

Known compound. ${ }^{5}$

${ }^{1} \mathbf{H}$ NMR $\left(500 \mathrm{MHz}, \mathrm{CDCl}_{3}\right): \delta 8.18(\mathrm{~s}, 1 \mathrm{H}), 7.33-7.23(\mathrm{~m}, 2 \mathrm{H}), 7.04(\mathrm{t}, J=7.6 \mathrm{~Hz}, 1 \mathrm{H})$, $6.88(\mathrm{~d}, J=7.7 \mathrm{~Hz}, 1 \mathrm{H}), 3.18(\mathrm{dd}, J=90.7,15.9 \mathrm{~Hz}, 2 \mathrm{H}), 2.17(\mathrm{~s}, 3 \mathrm{H}), 0.88(\mathrm{~s}, 9 \mathrm{H}),-0.06(\mathrm{~s}$, $3 \mathrm{H}),-0.25(\mathrm{~s}, 3 \mathrm{H})$.

${ }^{13}$ C NMR (126 MHz, $\left.\mathrm{CDCl}_{3}\right): \delta$ 205.02, 178.00, 140.69, 130.38, 129.87, 124.63, 122.57, $110.24,75.41,52.53,31.24,25.58,18.07,-3.78,-4.22$.

Optical Rotation: $[\alpha]_{D}^{24}=-19.000\left(\mathrm{c}=0.14, \mathrm{CHCl}_{3}\right)$.

94:6 er. HPLC condition: Chiralpak IE, $n$-hexane $/ i-\mathrm{PrOH}=90: 10$, flow rate $=1 \mathrm{ml} / \mathrm{min}$, wavelength $=254 \mathrm{~nm}, \mathrm{t}_{\mathrm{R}}=10.418 \mathrm{~min}$ for major isomer, $\mathrm{t}_{\mathrm{R}}=13.995 \mathrm{~min}$ for minor isomer. 

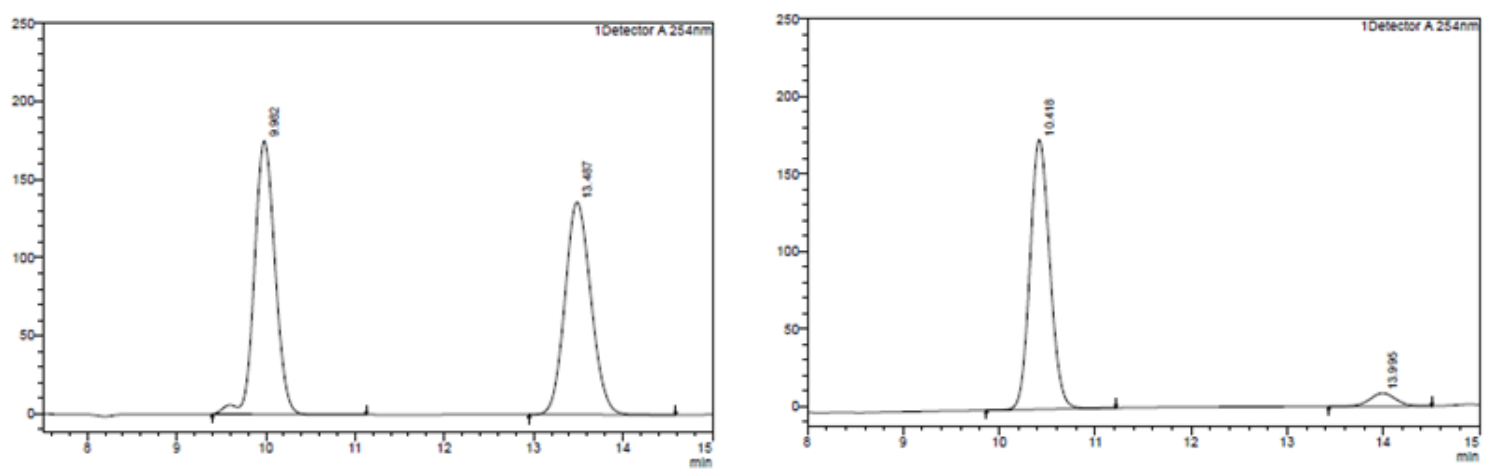

\begin{tabular}{|c|c|c|c|c|}
\hline \multirow{2}{*}{\multicolumn{5}{|c|}{ Detector A 254nm }} \\
\hline & & Area & Height & Conc \\
\hline 1 & 9.982 & 2820364 & 174967 & 50.553 \\
\hline 2 & 13.487 & 2758686 & 136038 & 49.447 \\
\hline Total & & 5579050 & 311005 & \\
\hline
\end{tabular}

\begin{tabular}{|c|c|c|c|c|}
\hline \\
\hline \multicolumn{2}{|c|}{$\begin{array}{l}\text { Detector A } 254 \mathrm{~nm} \\
\text { Peak\# Ret. Time }\end{array}$} & Area & Height & Conc. \\
\hline 1 & 10.418 & 2488654 & 173838 & 94.075 \\
\hline 2 & 13.995 & 156744 & 8419 & 5.925 \\
\hline Total & & 2645398 & 182257 & \\
\hline
\end{tabular}


7. NMR spectra of the products and derivatives

NMR spectra of the products:
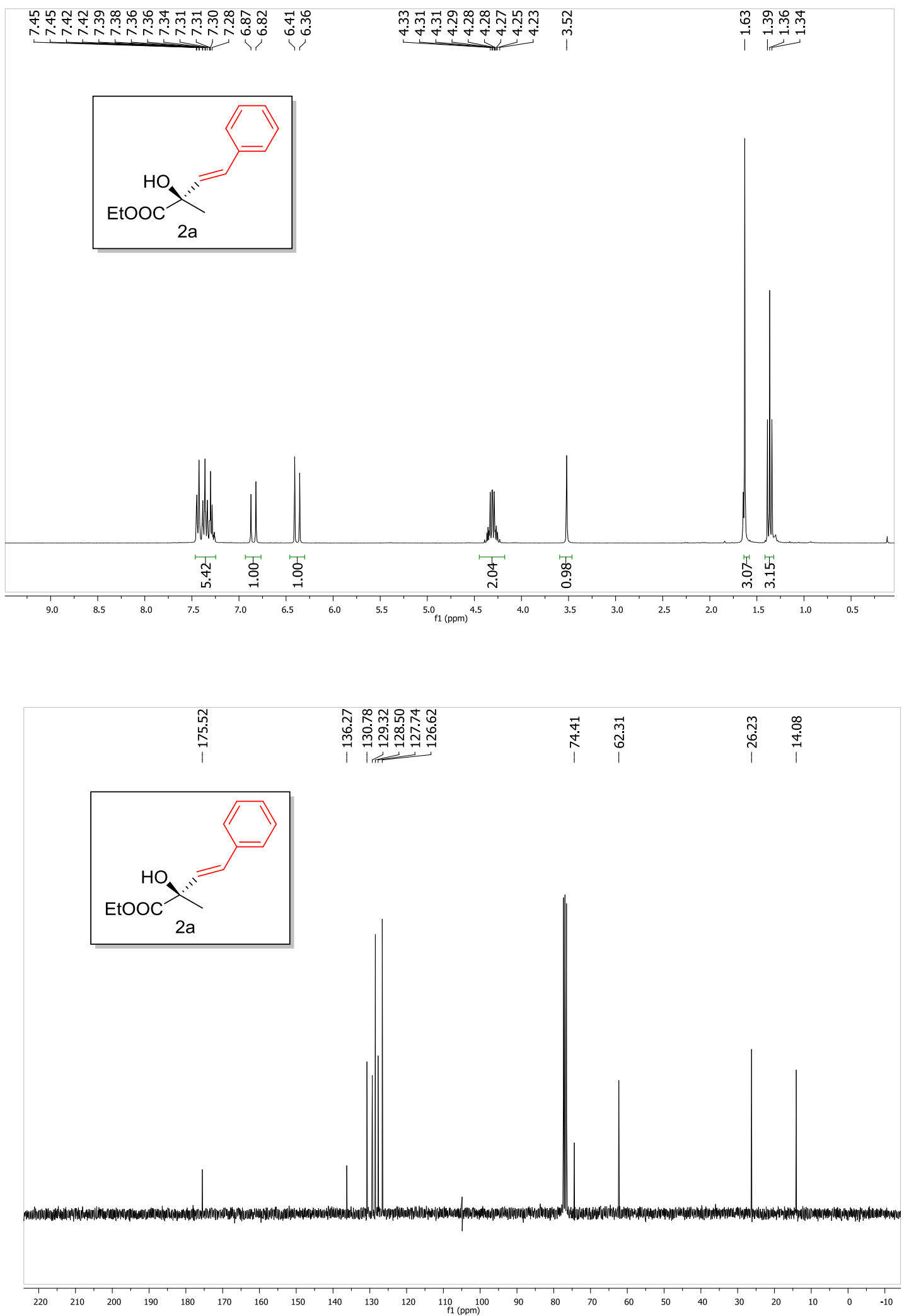

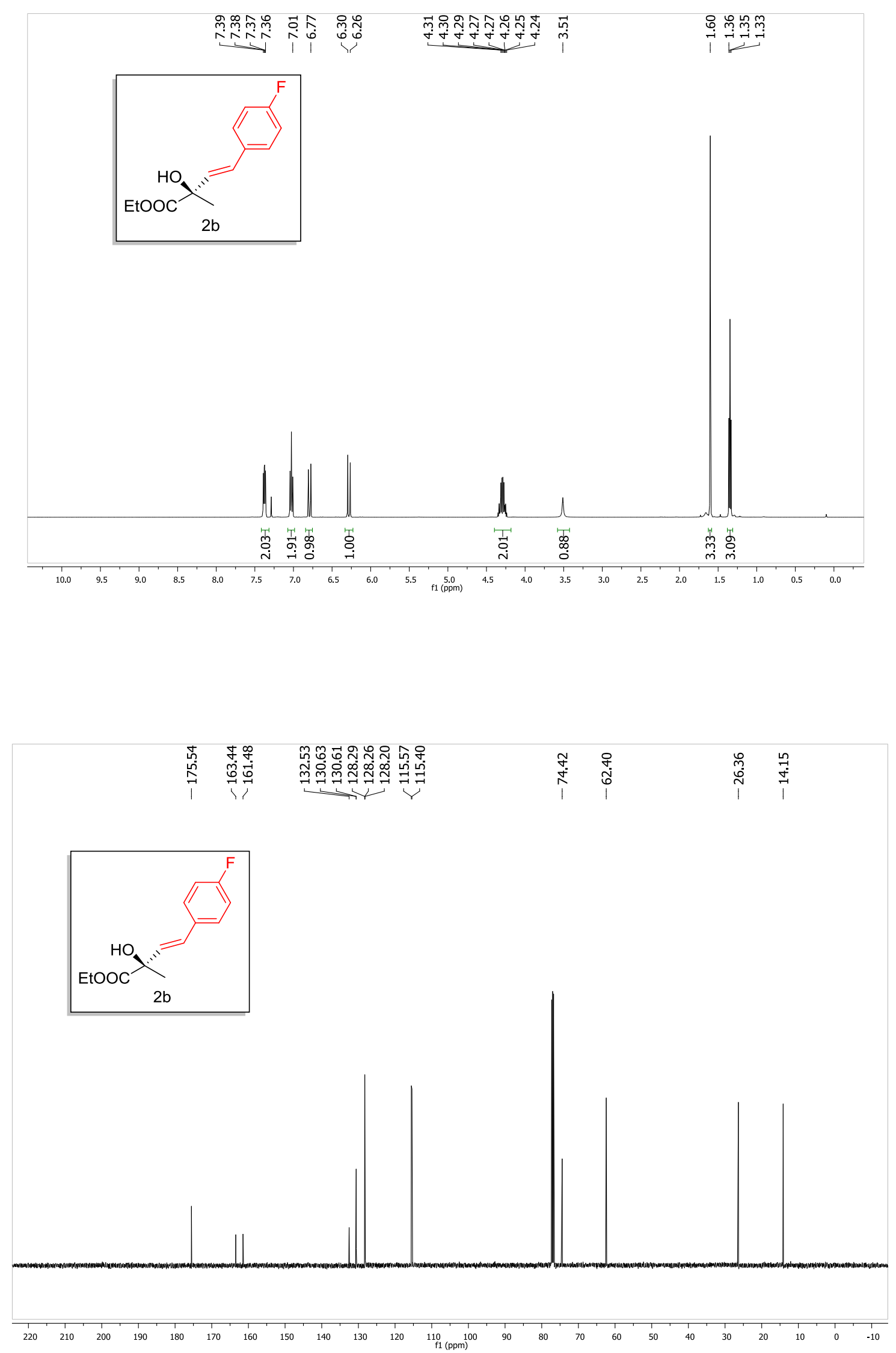


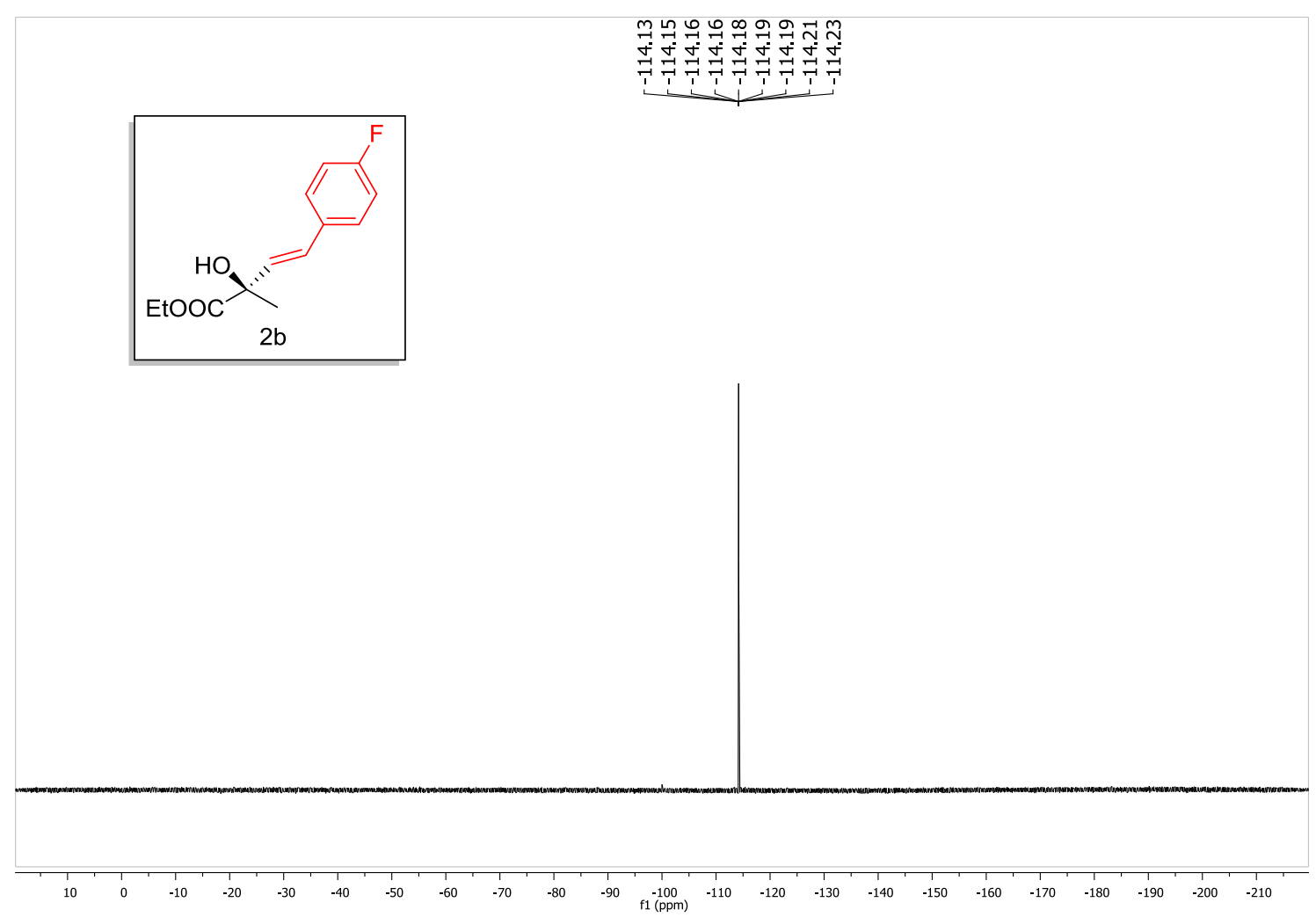



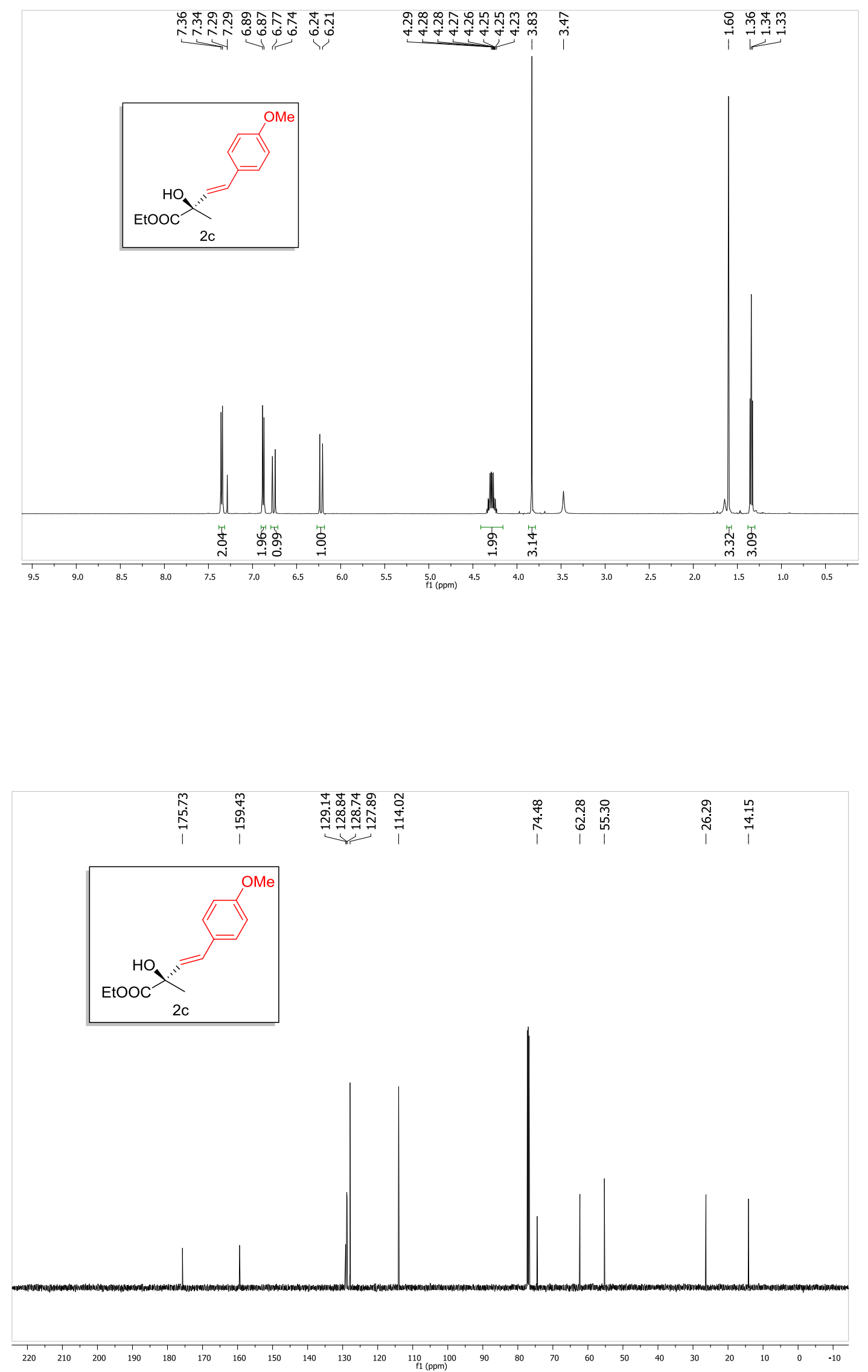

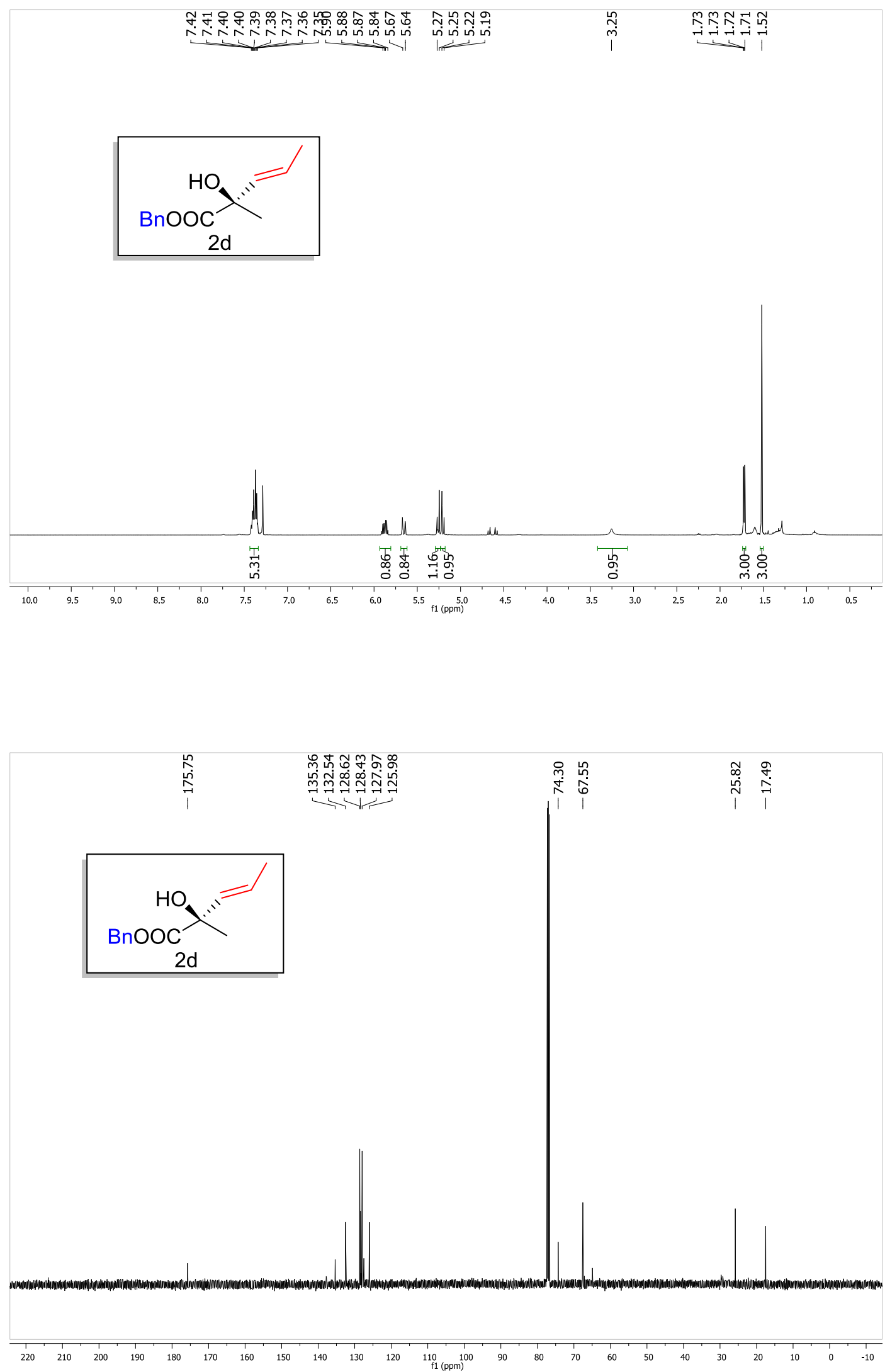

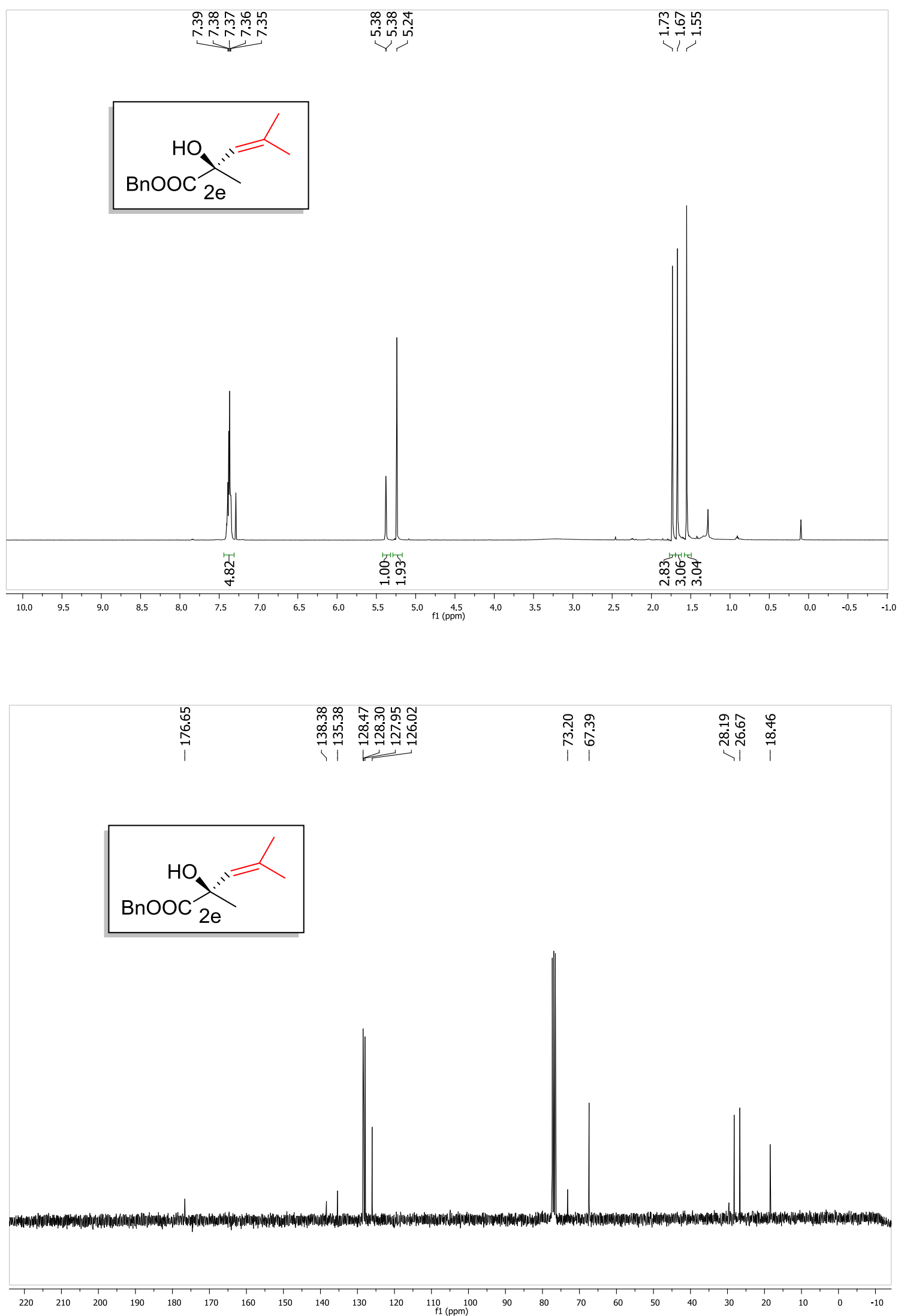


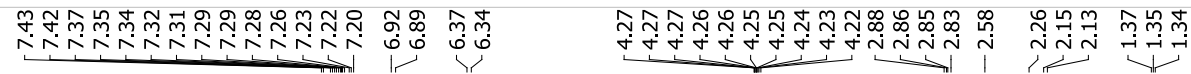

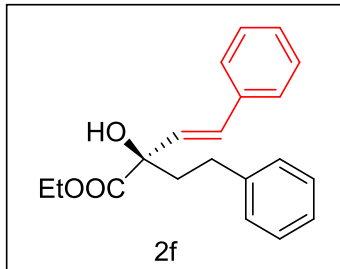

2

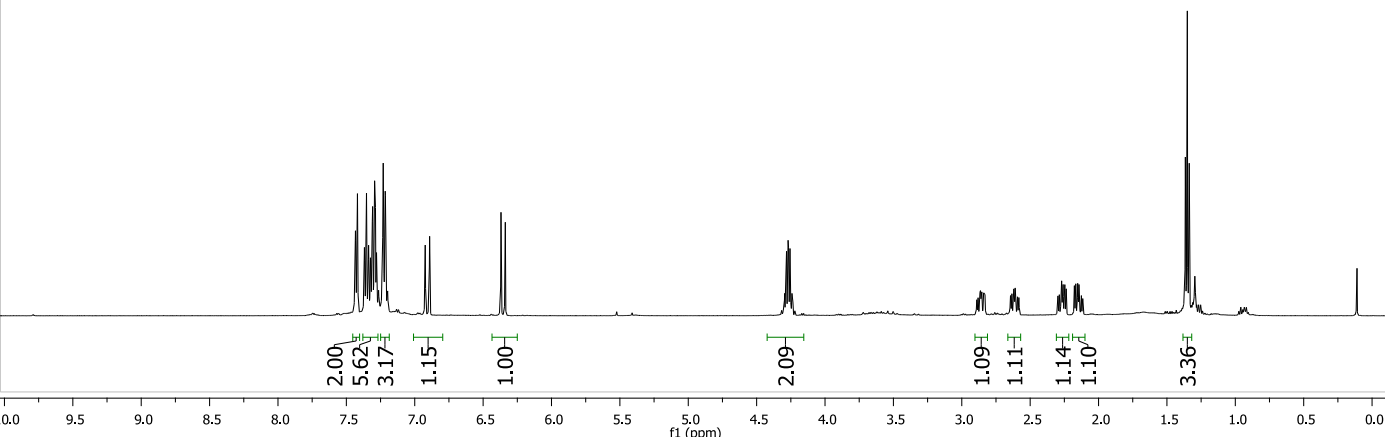

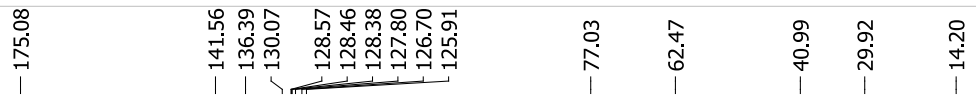
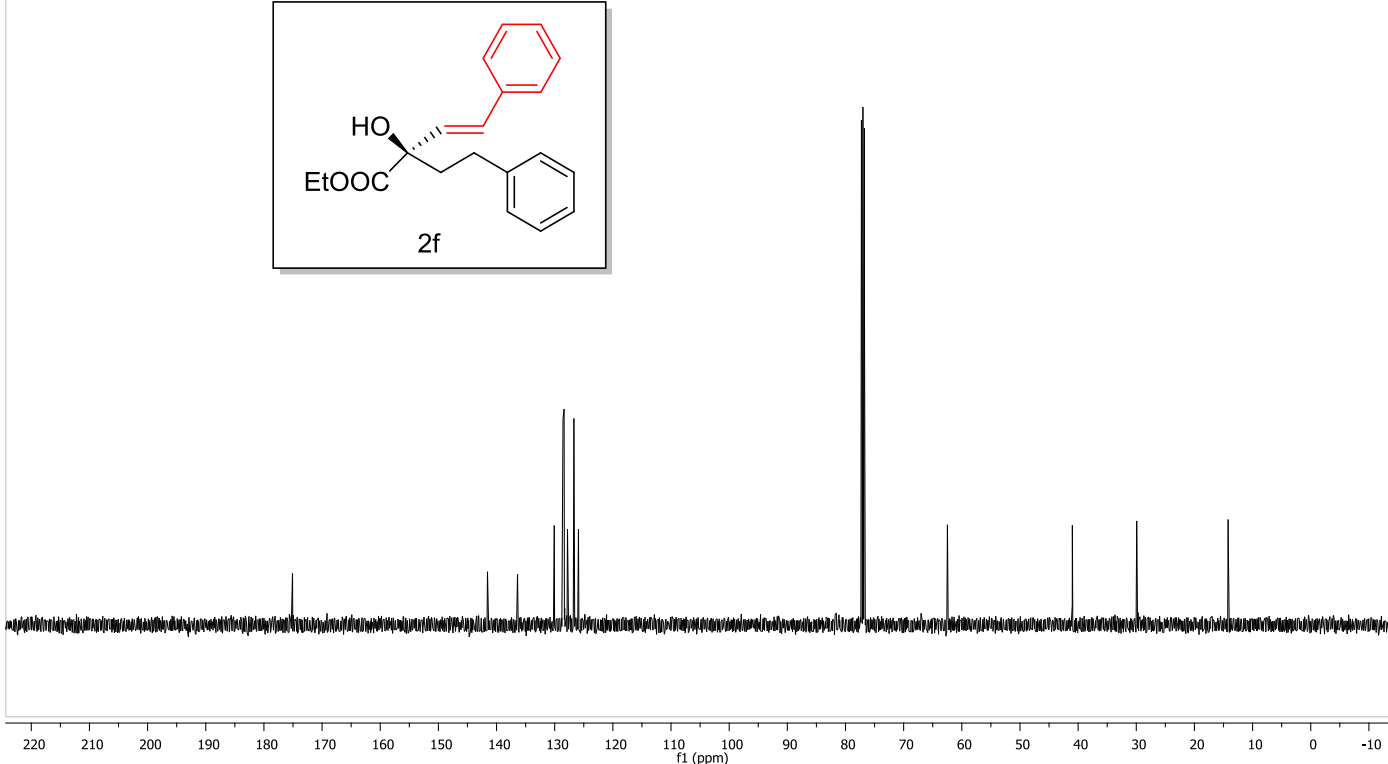

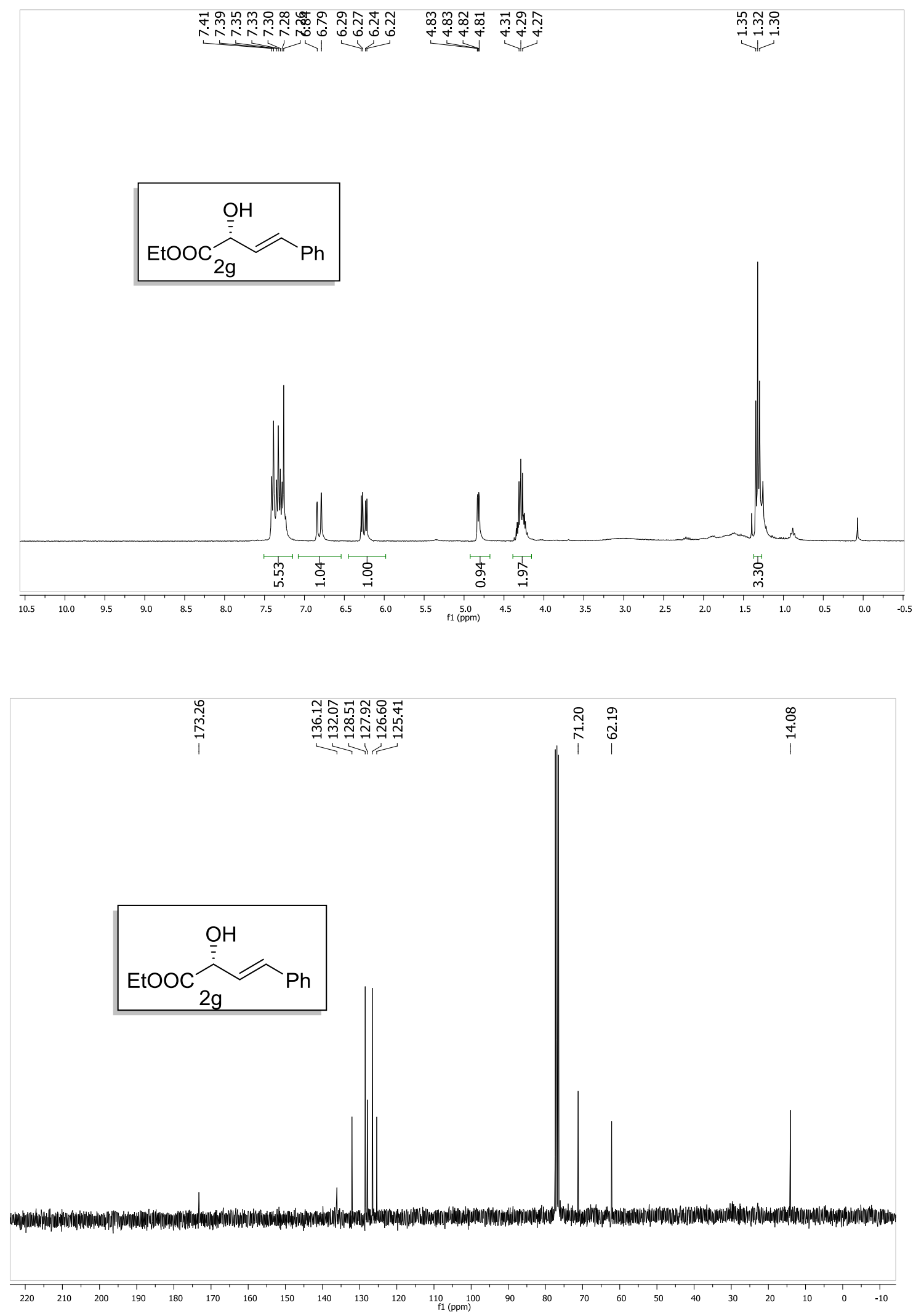

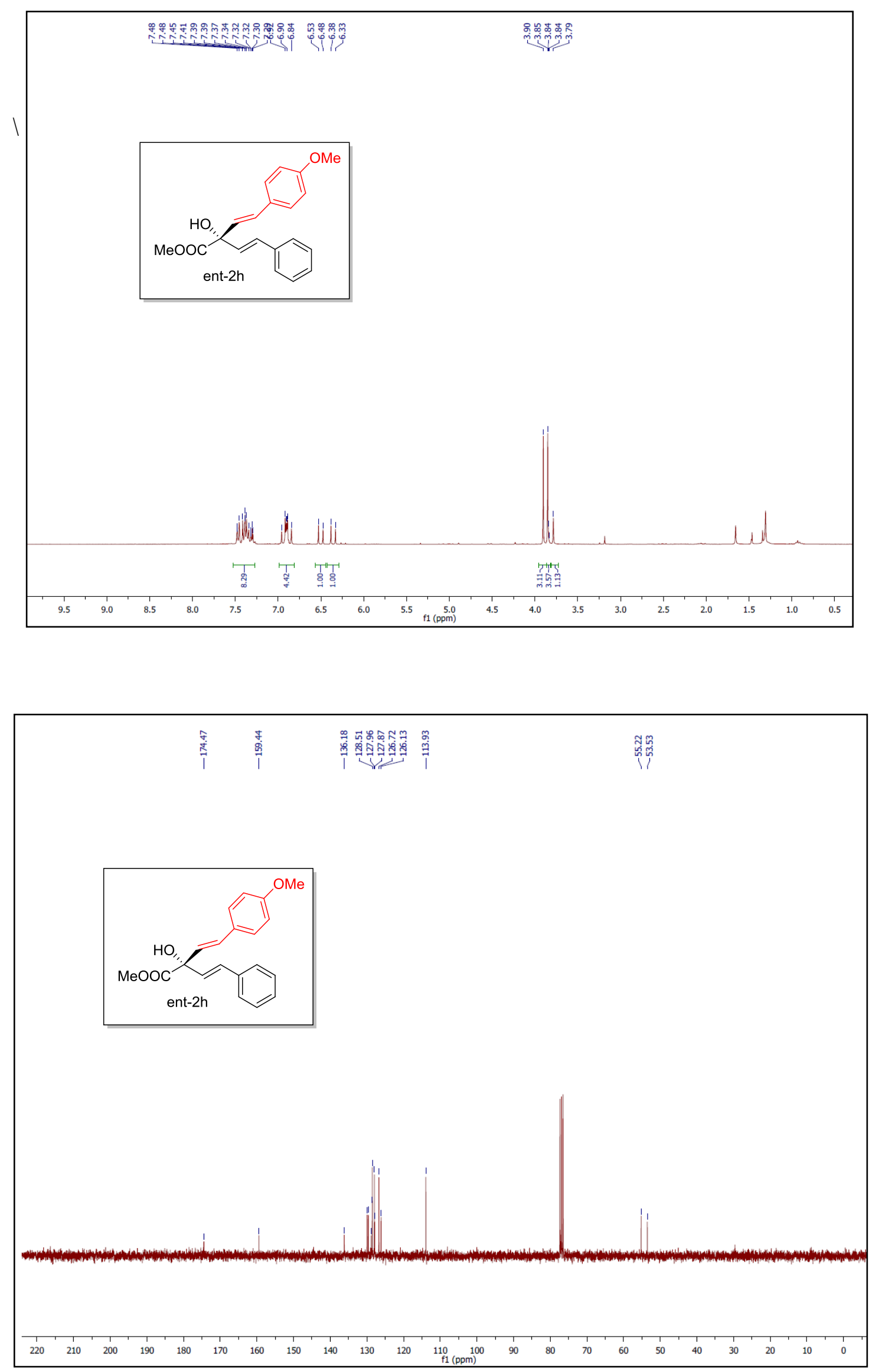

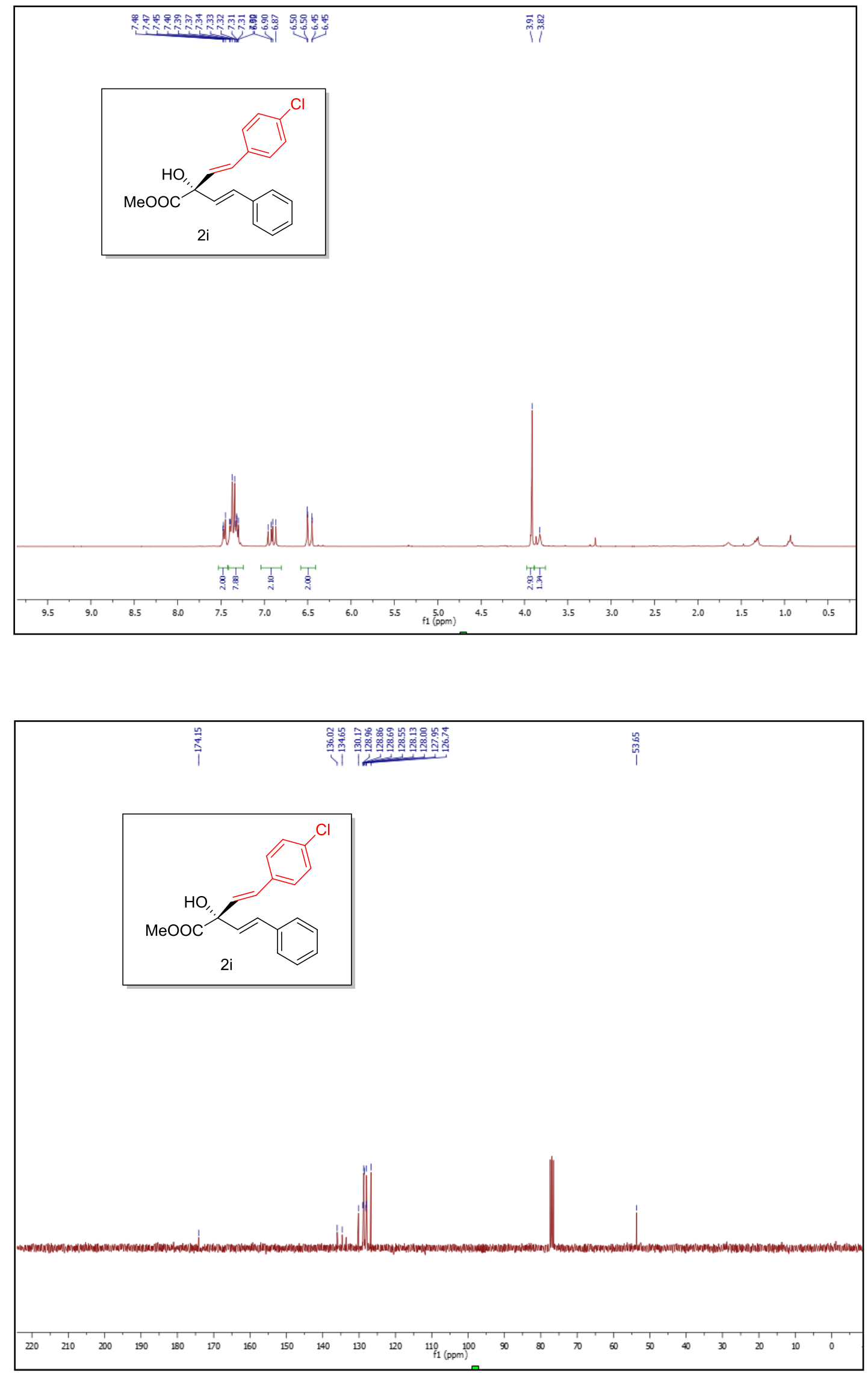

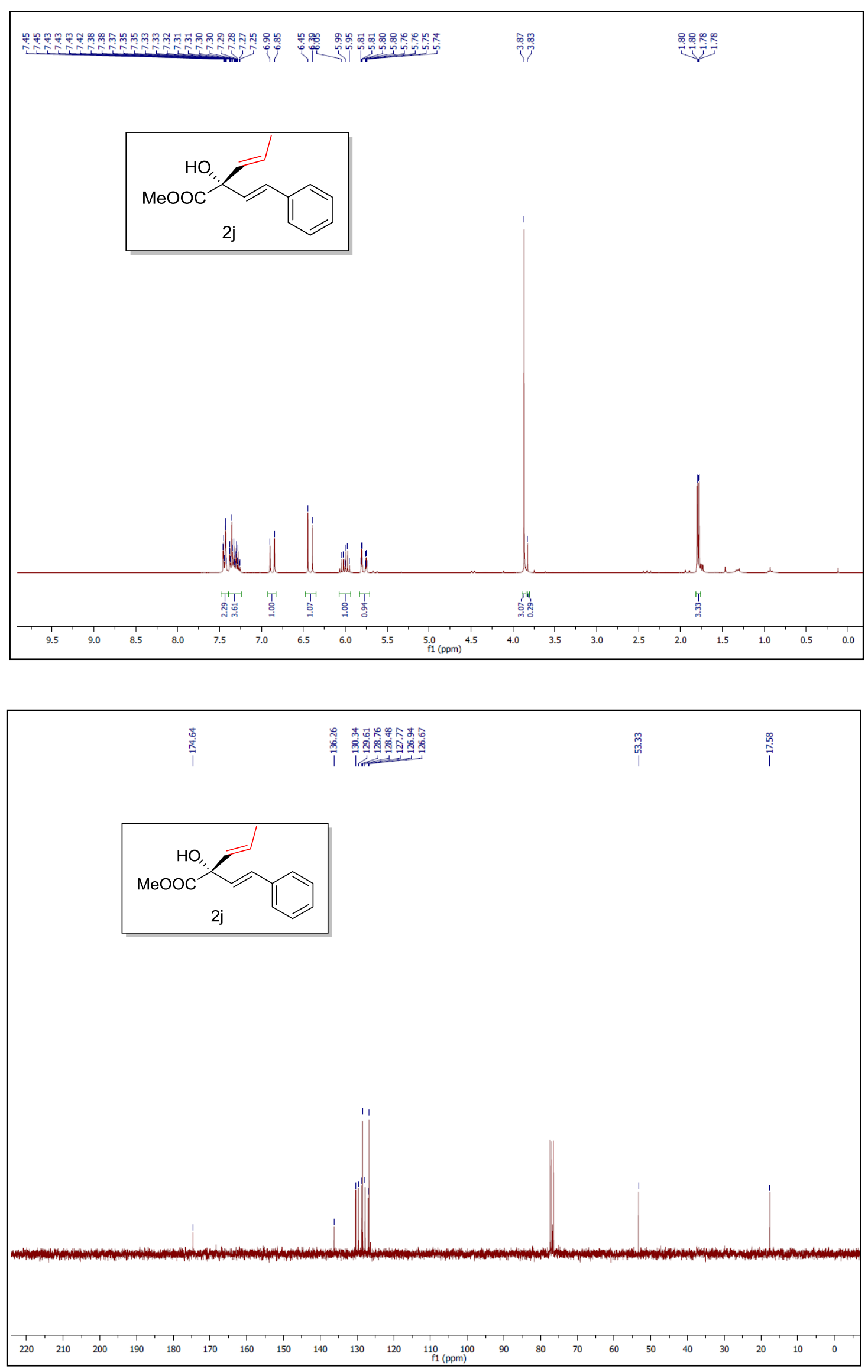

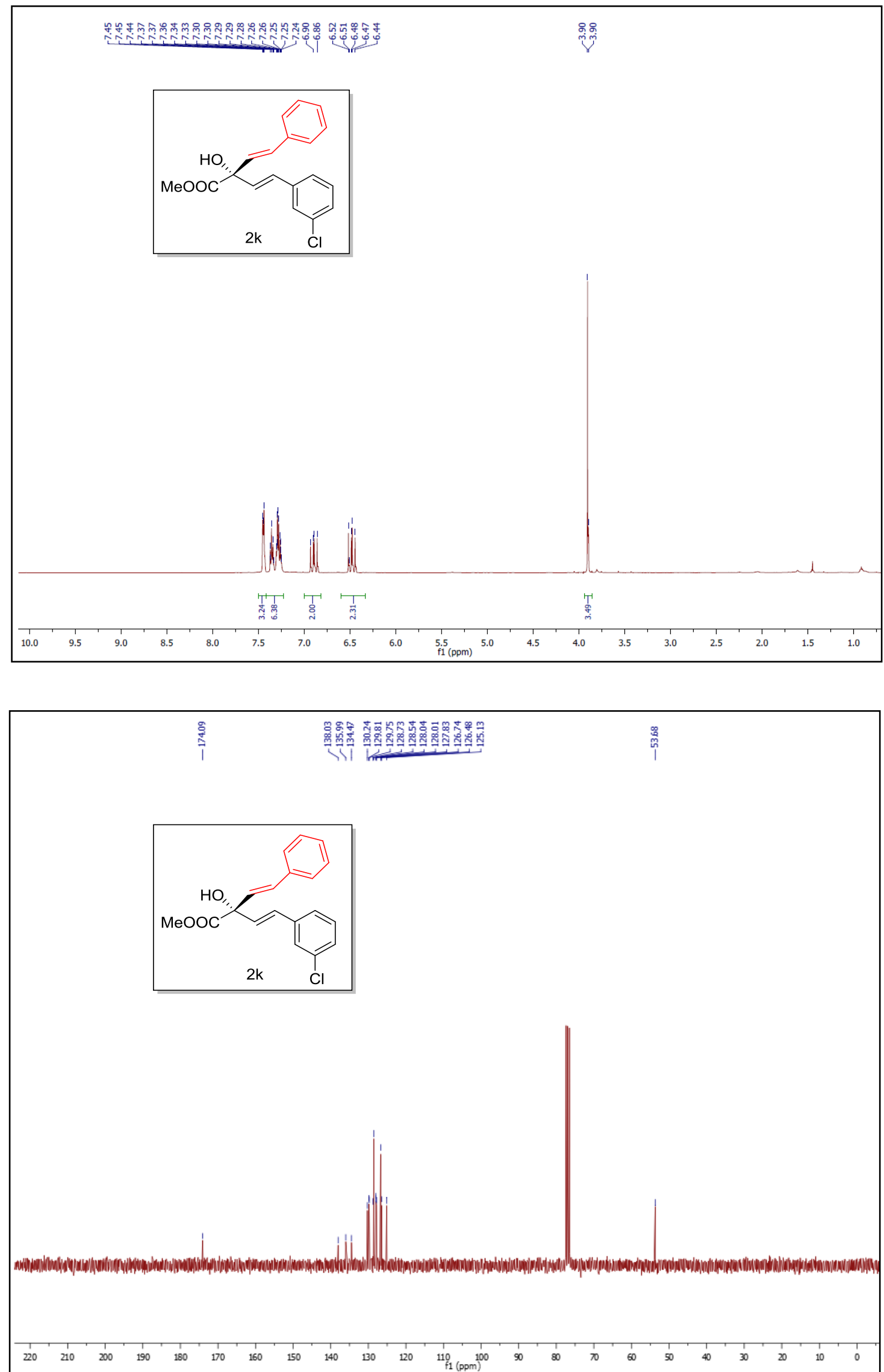

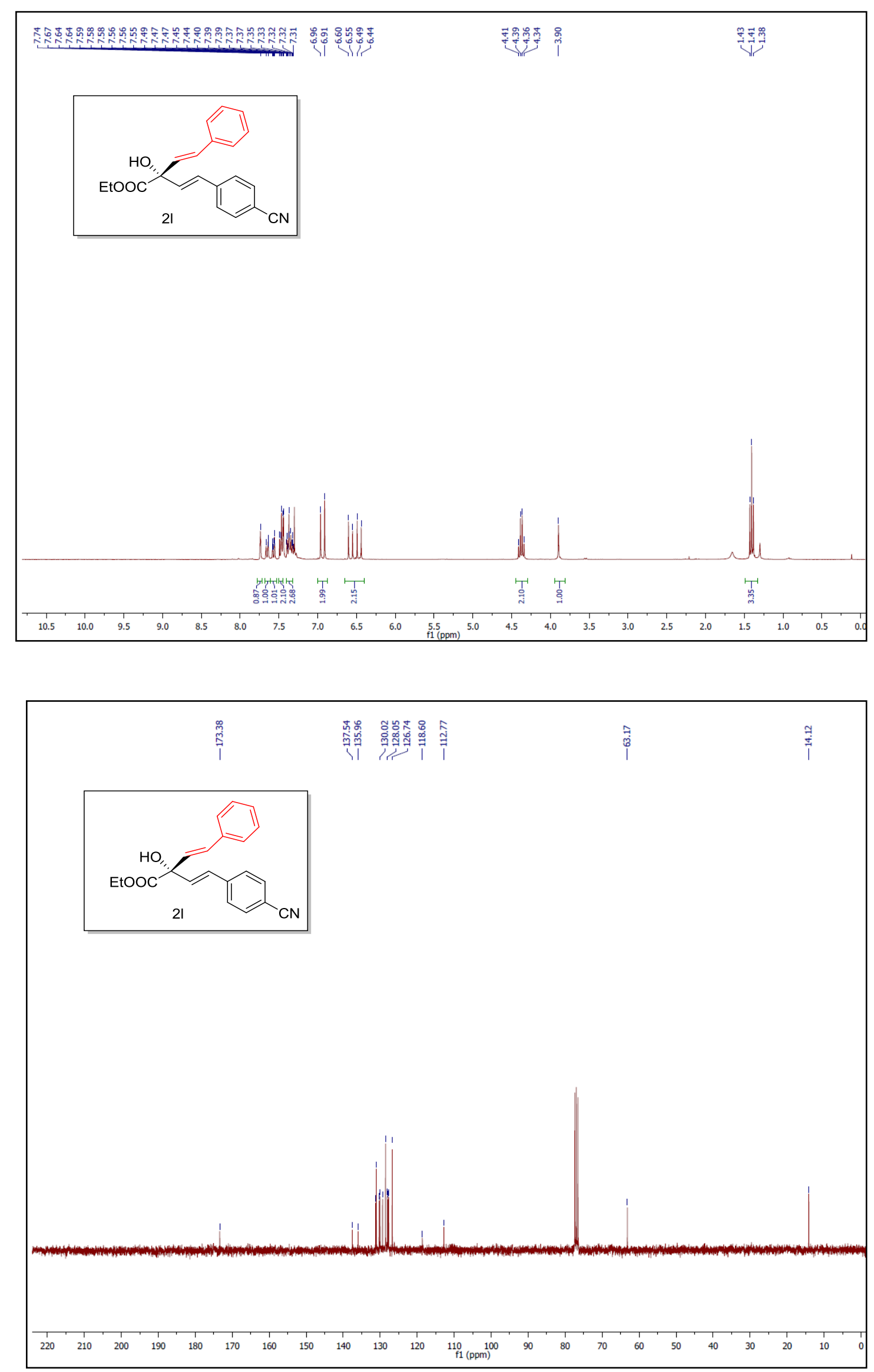

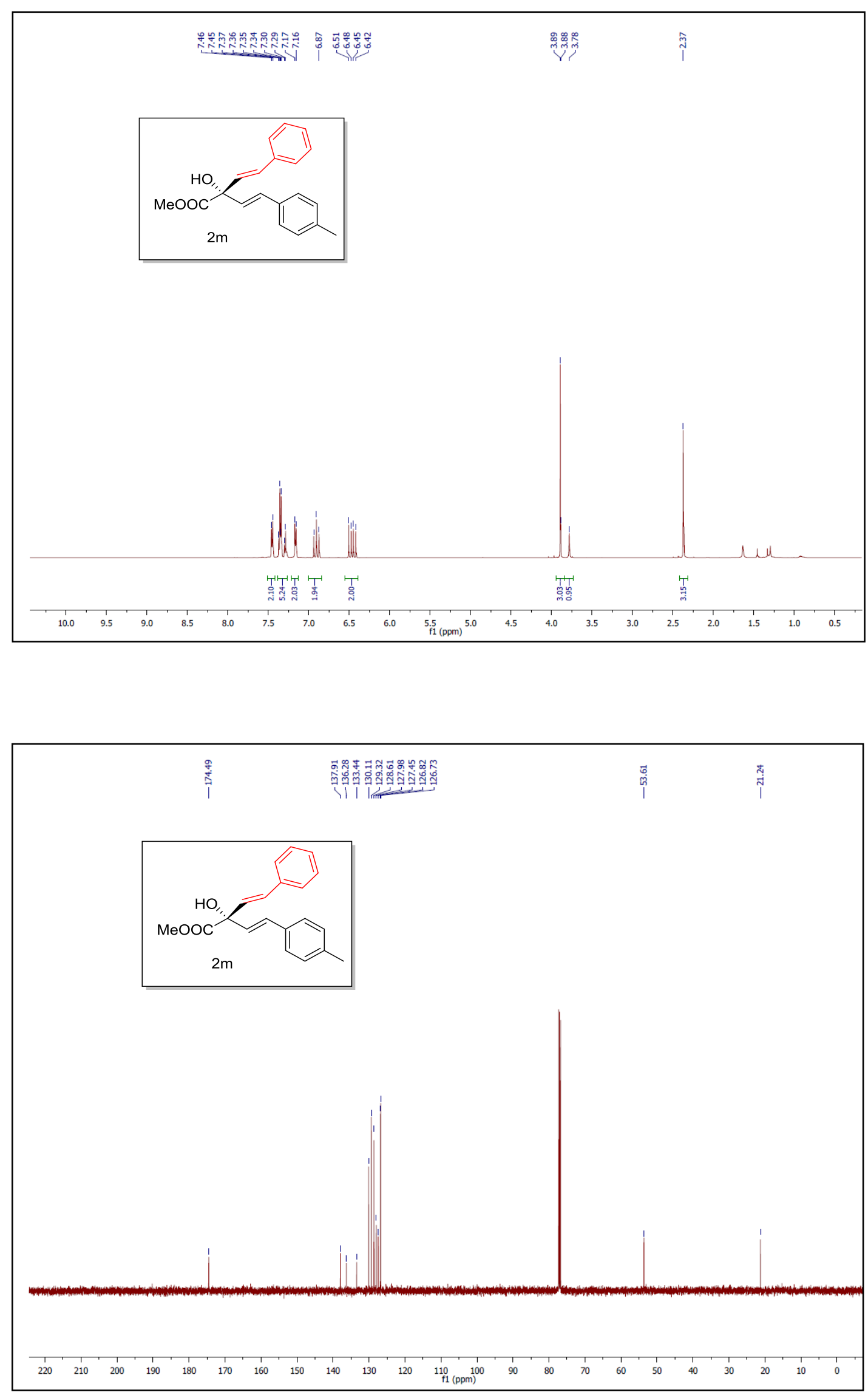

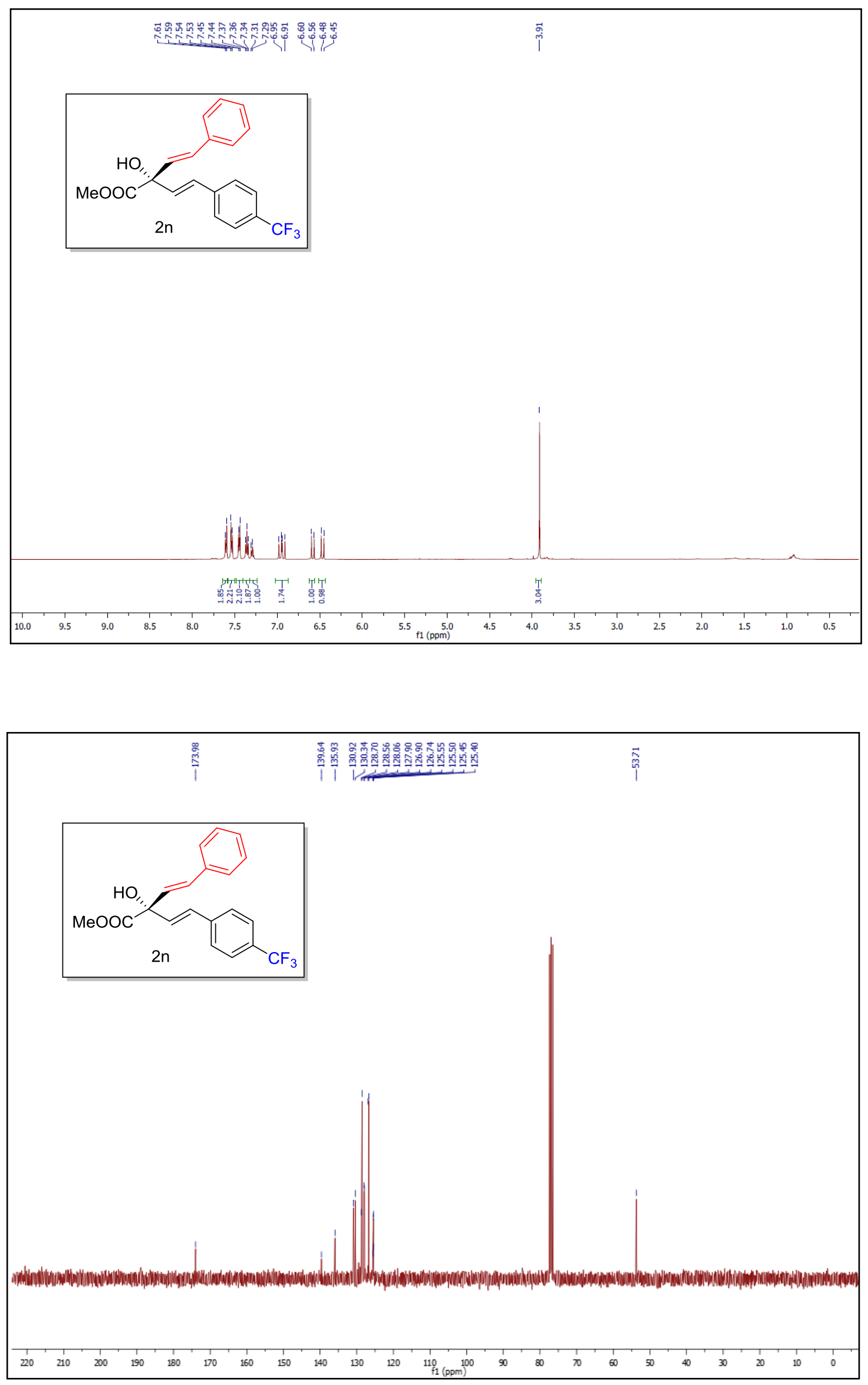


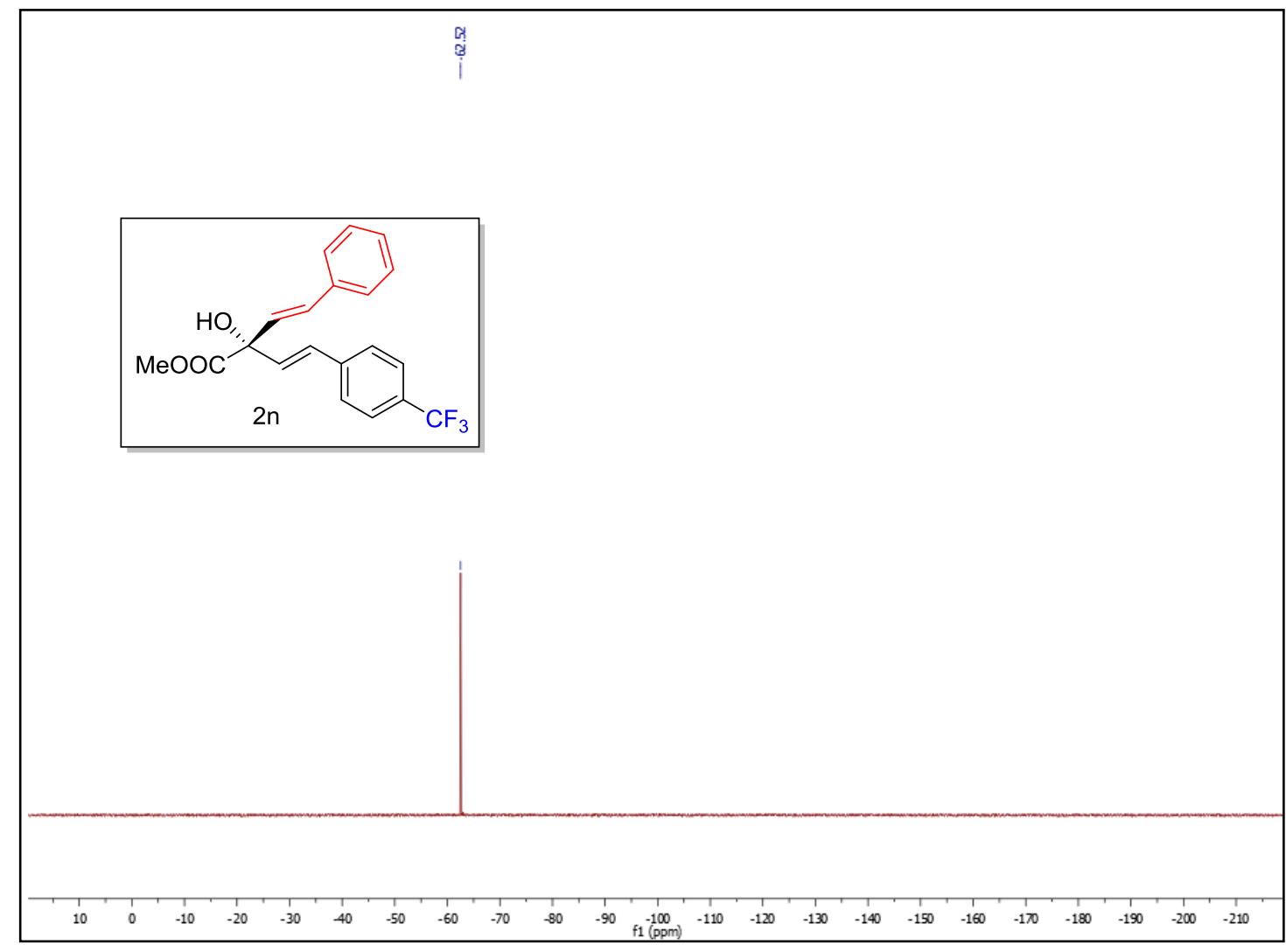



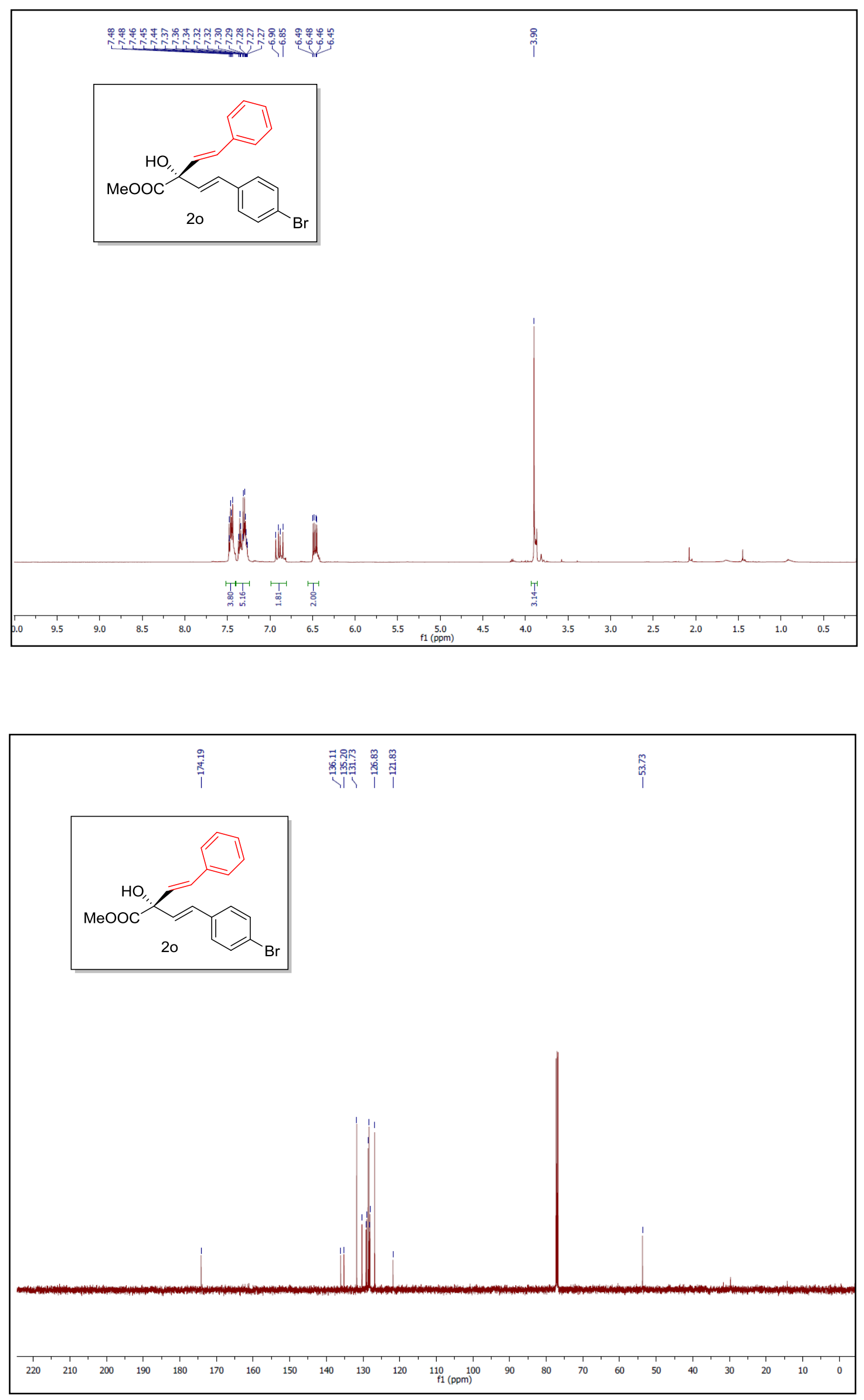

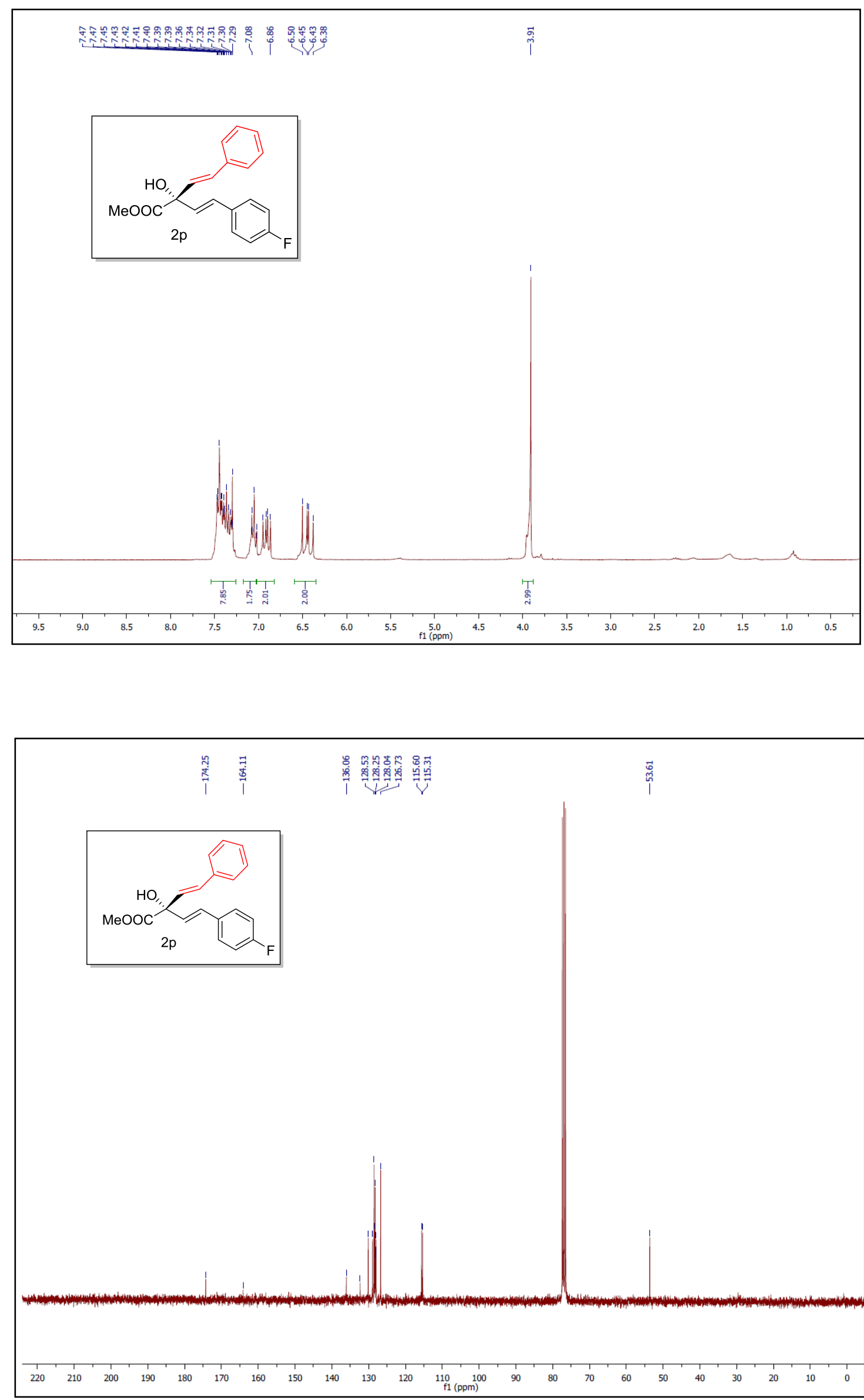


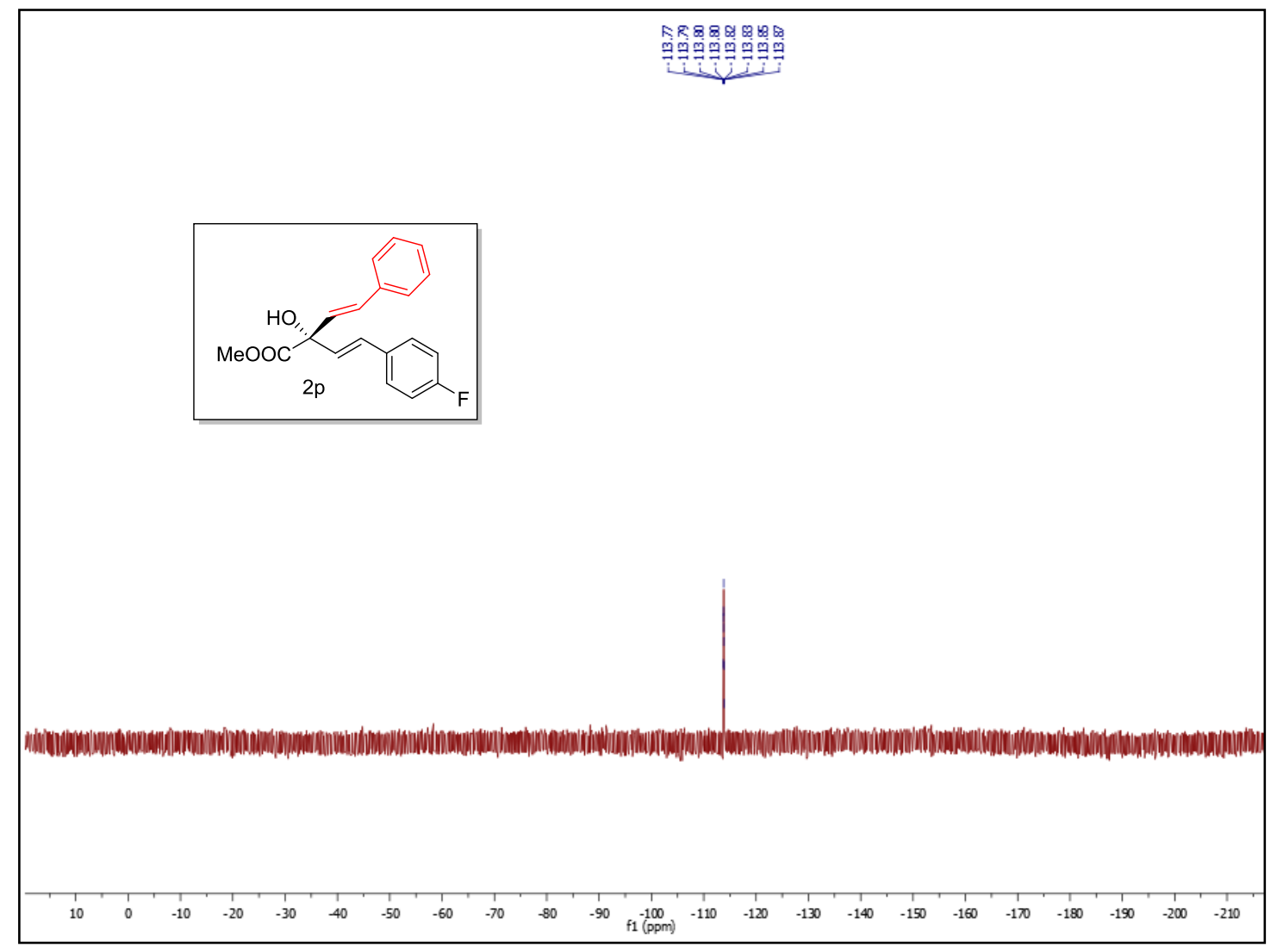



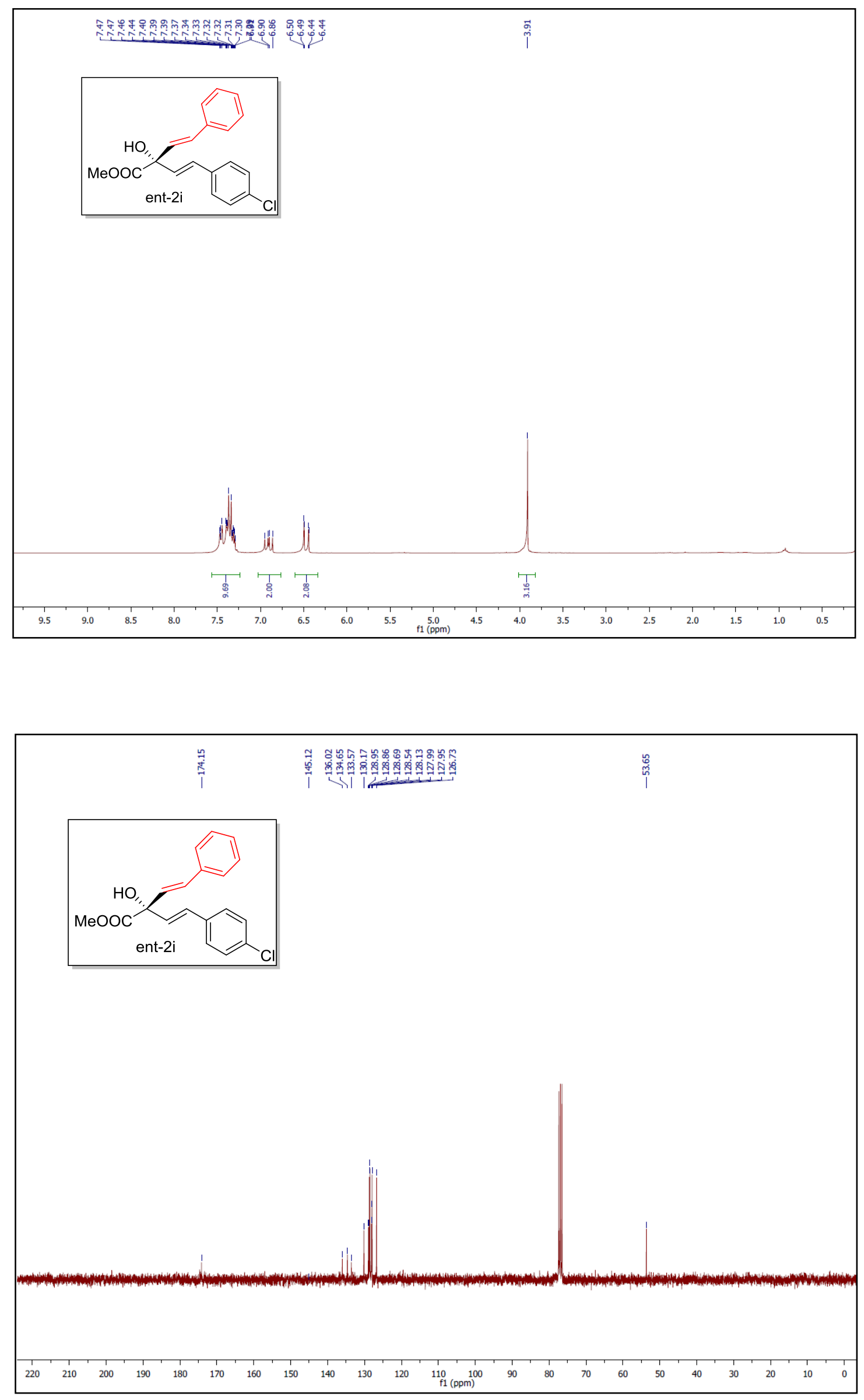

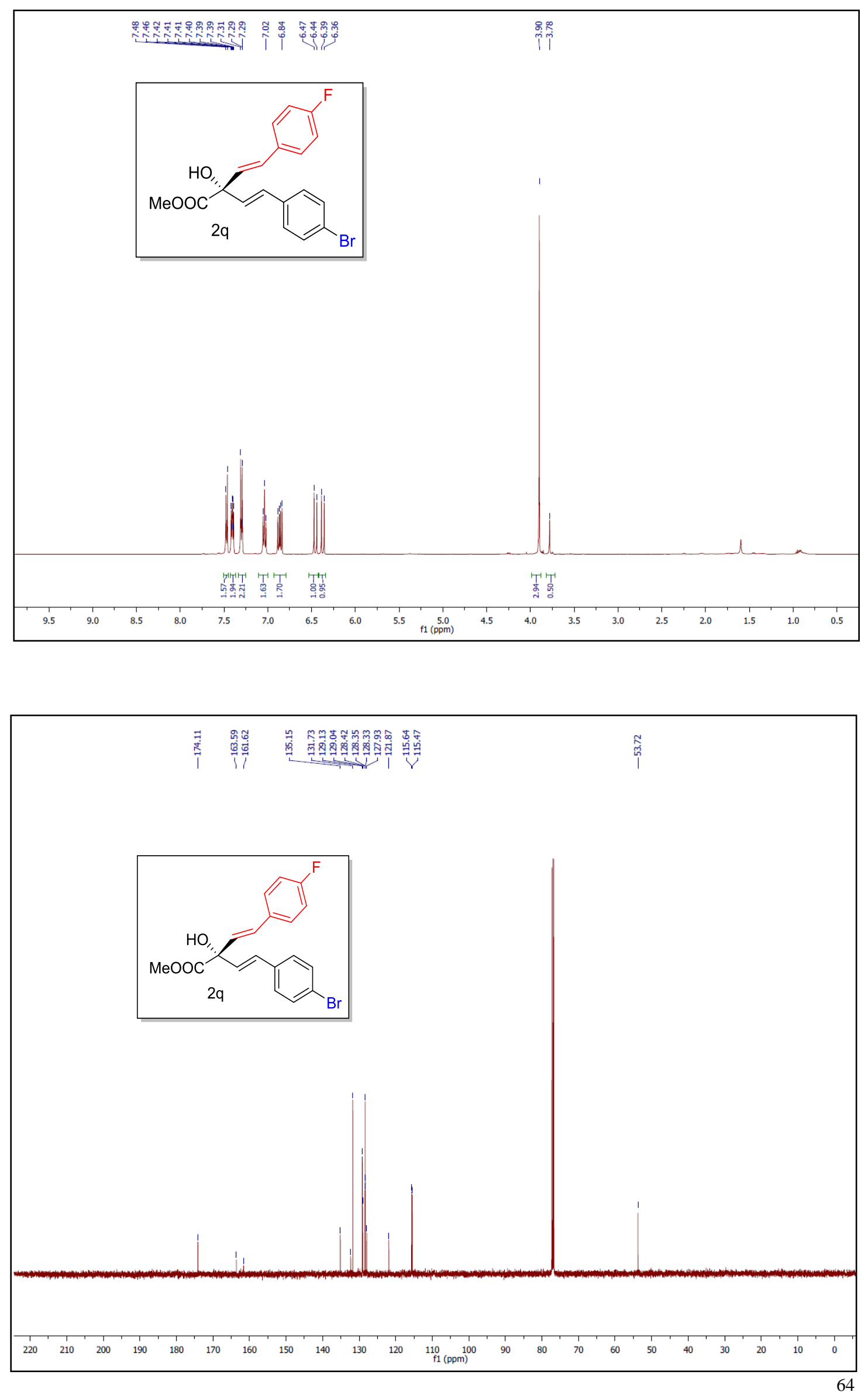


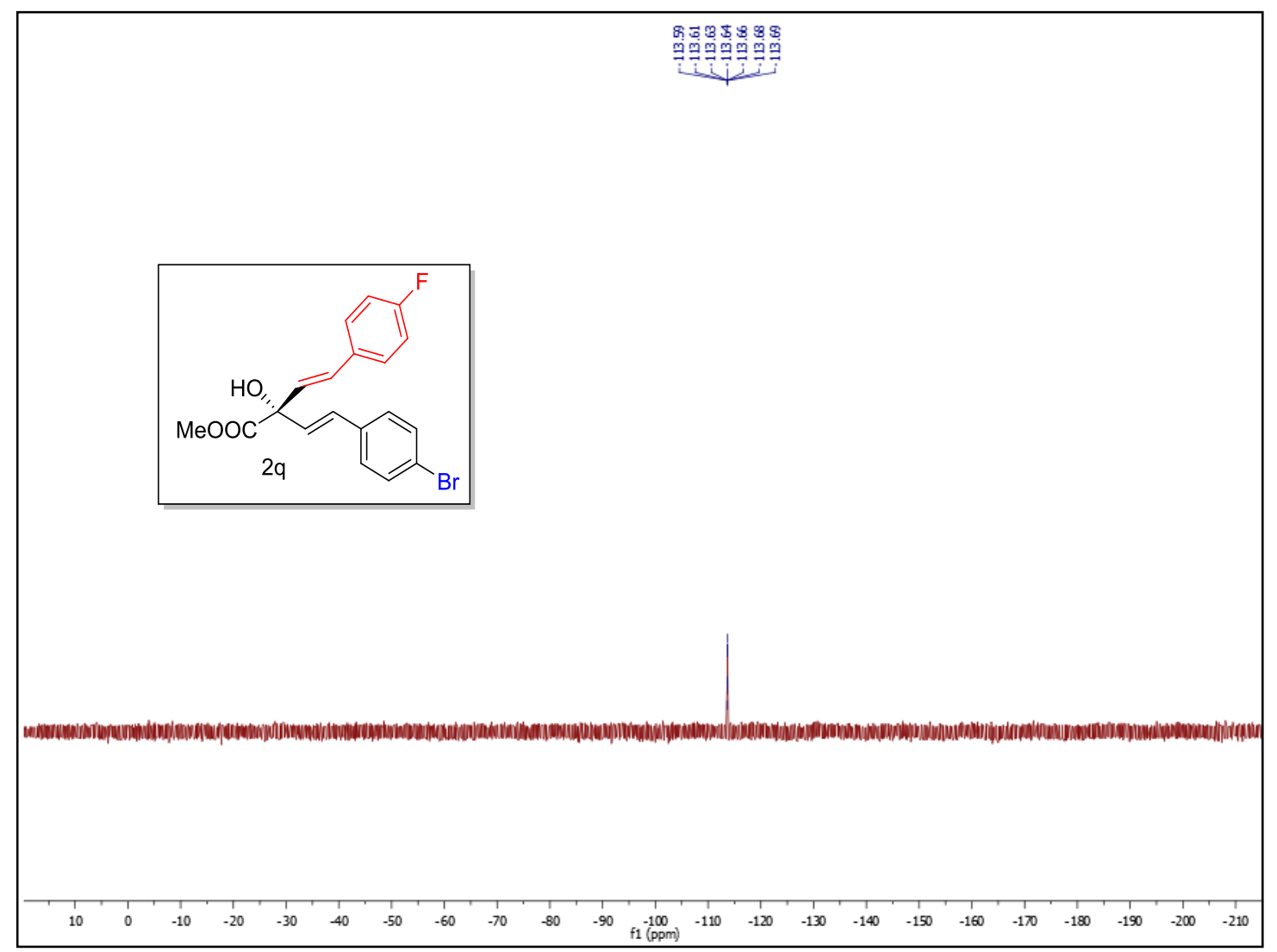



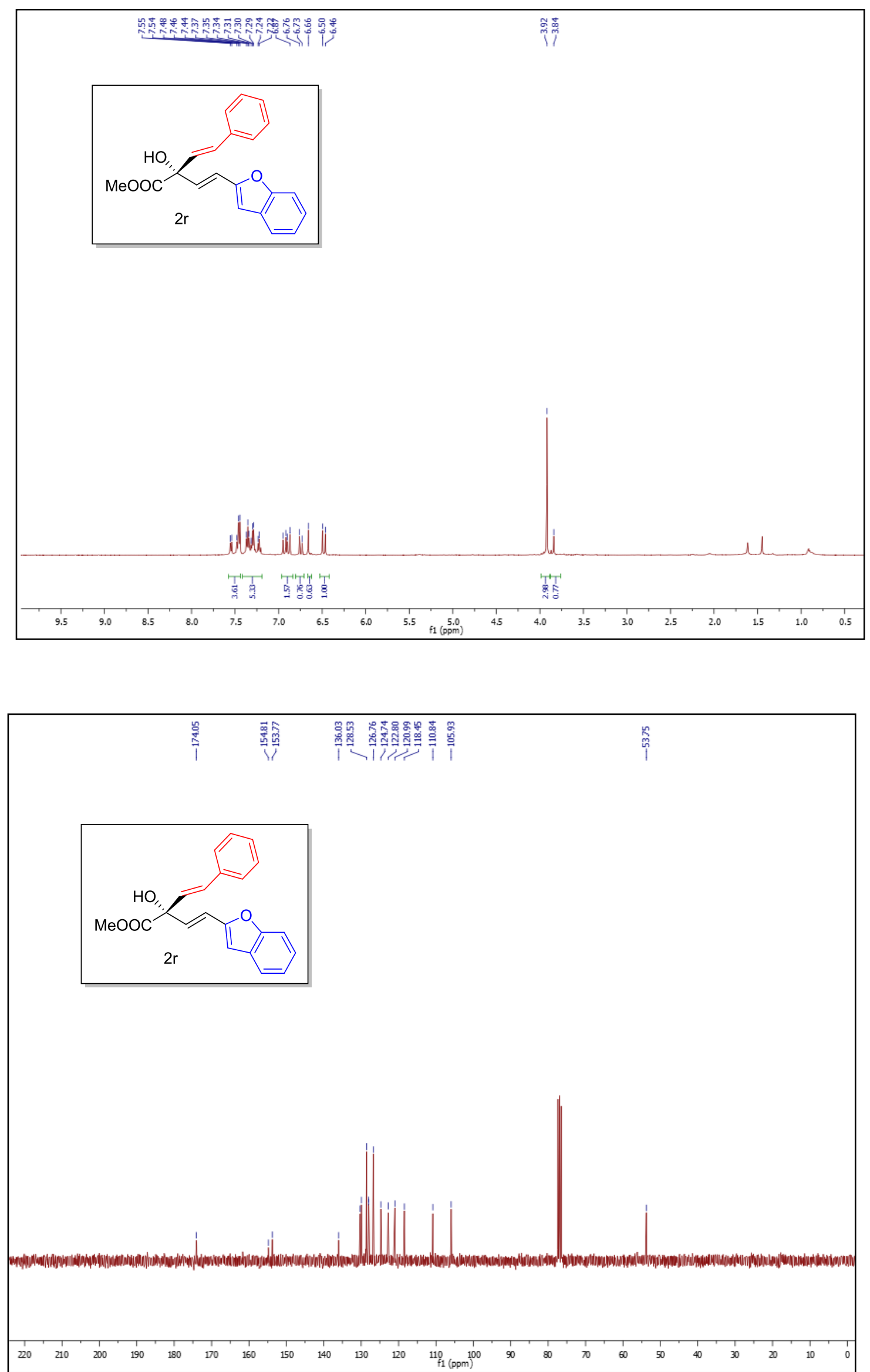

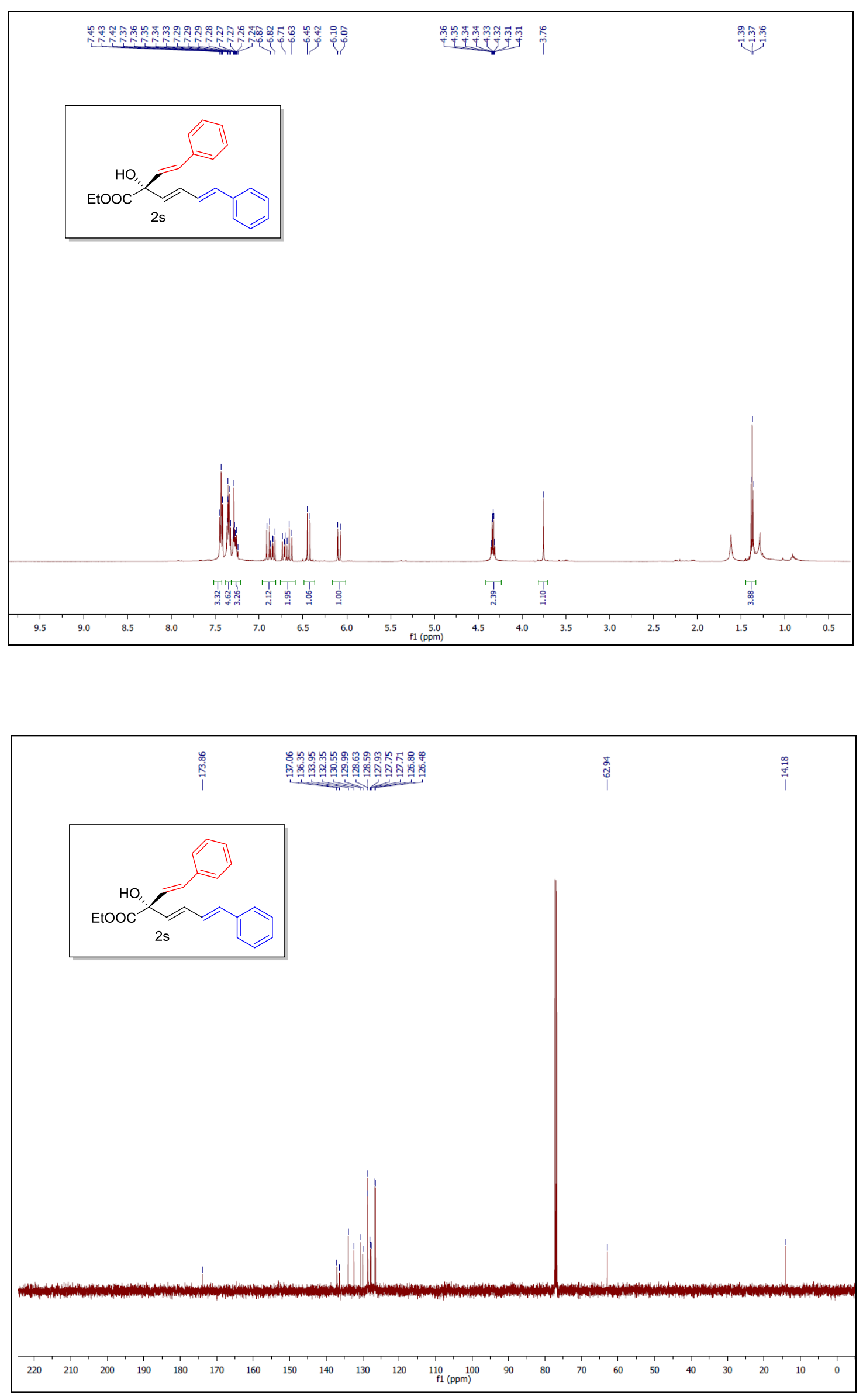


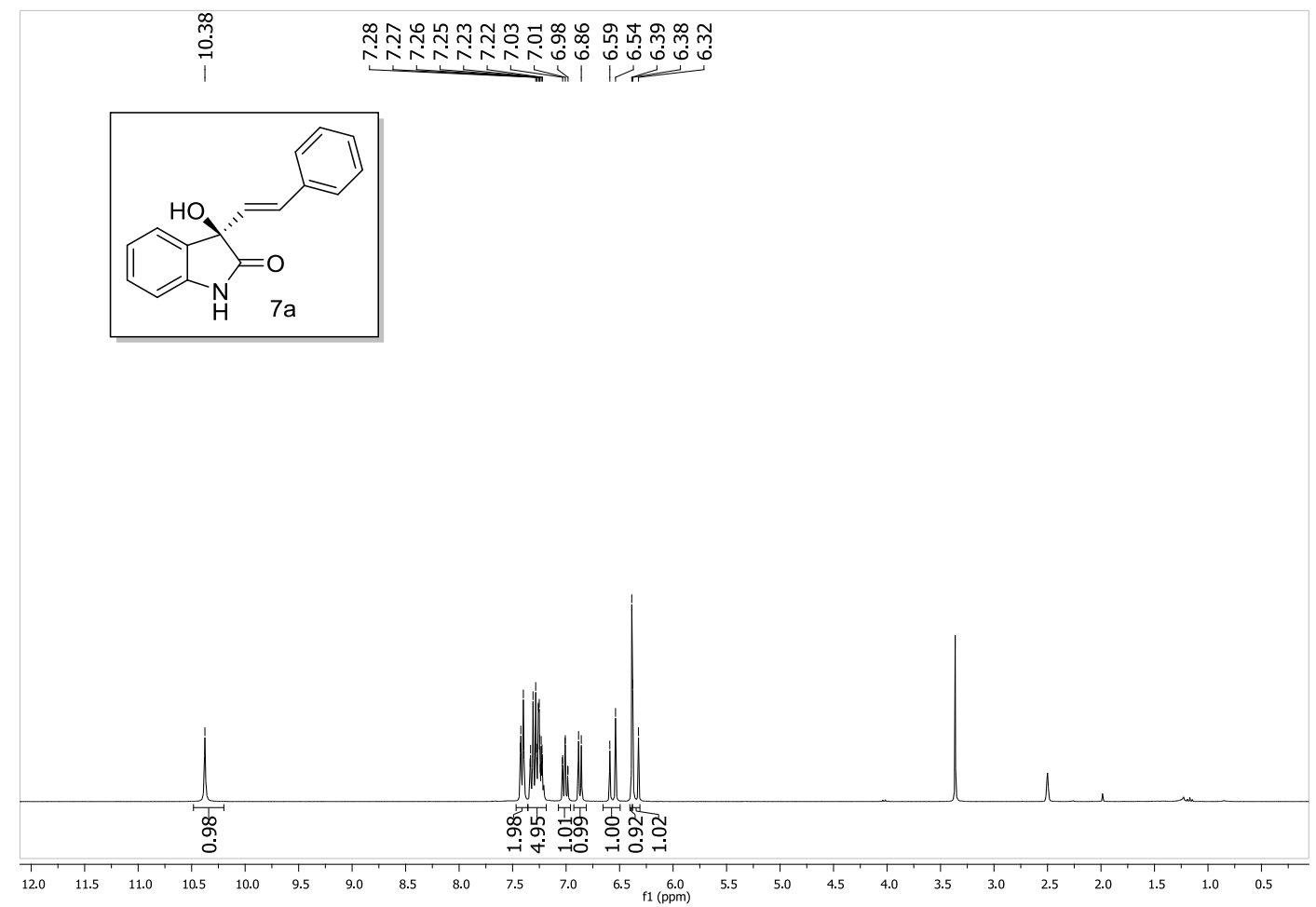

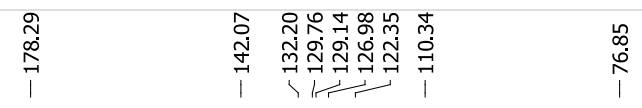
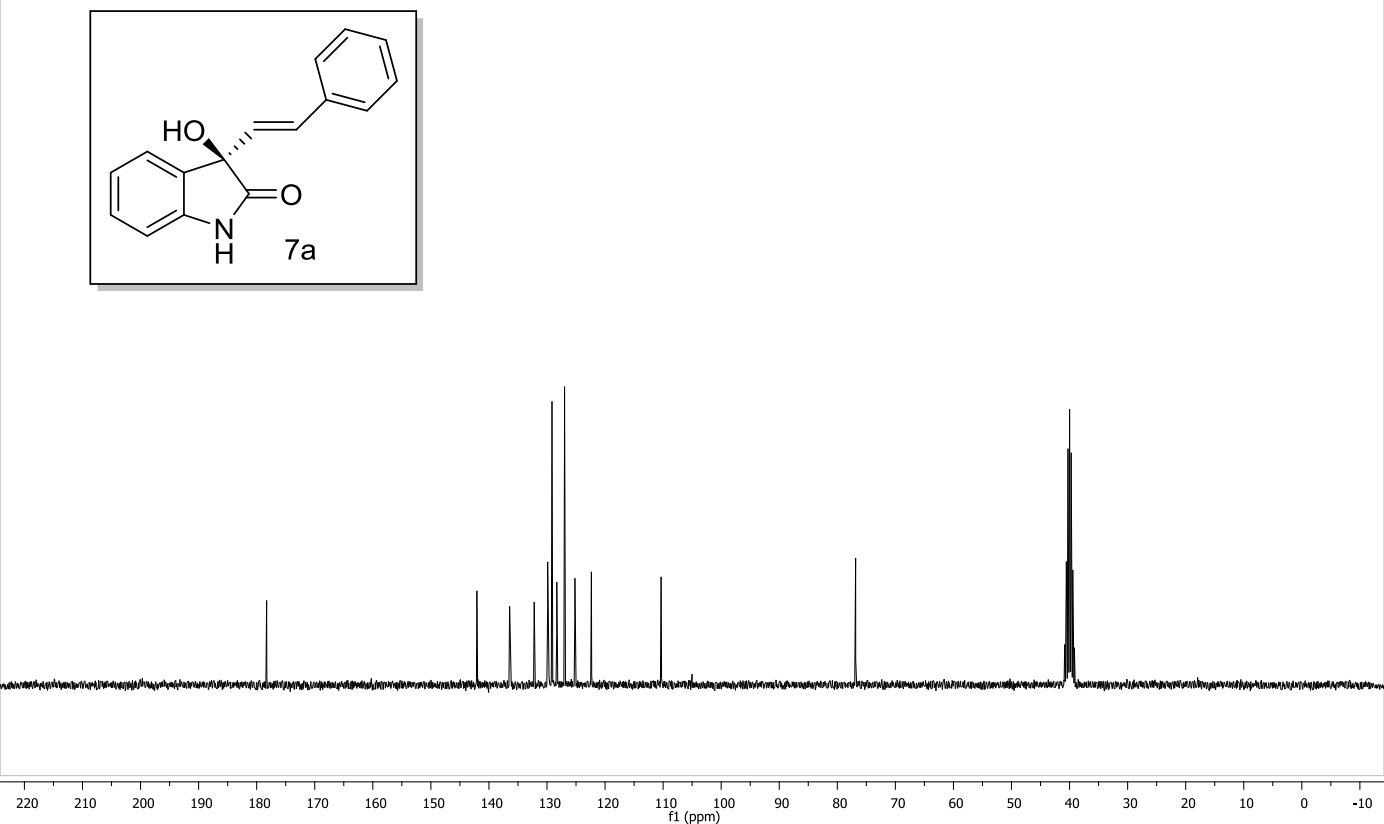

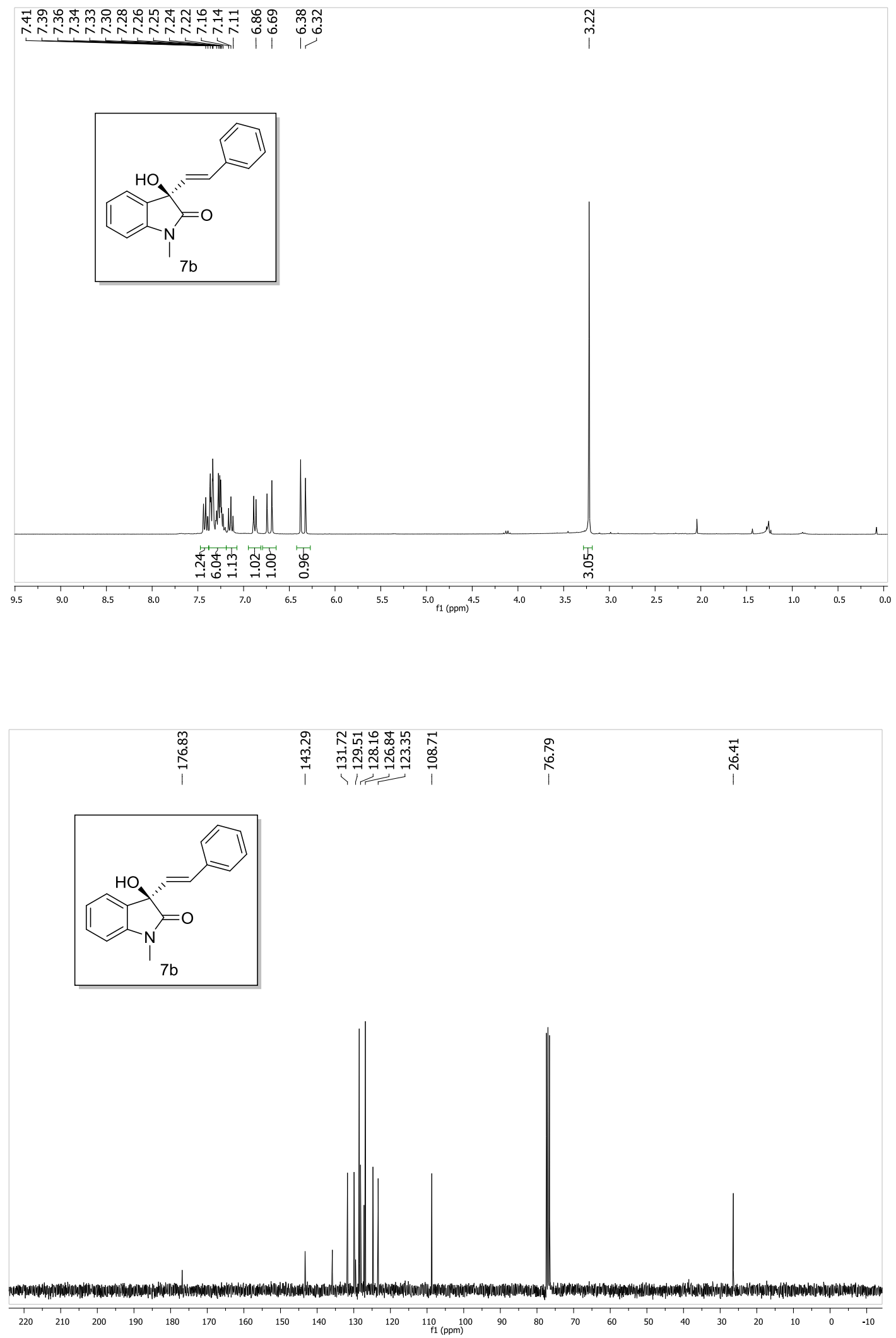

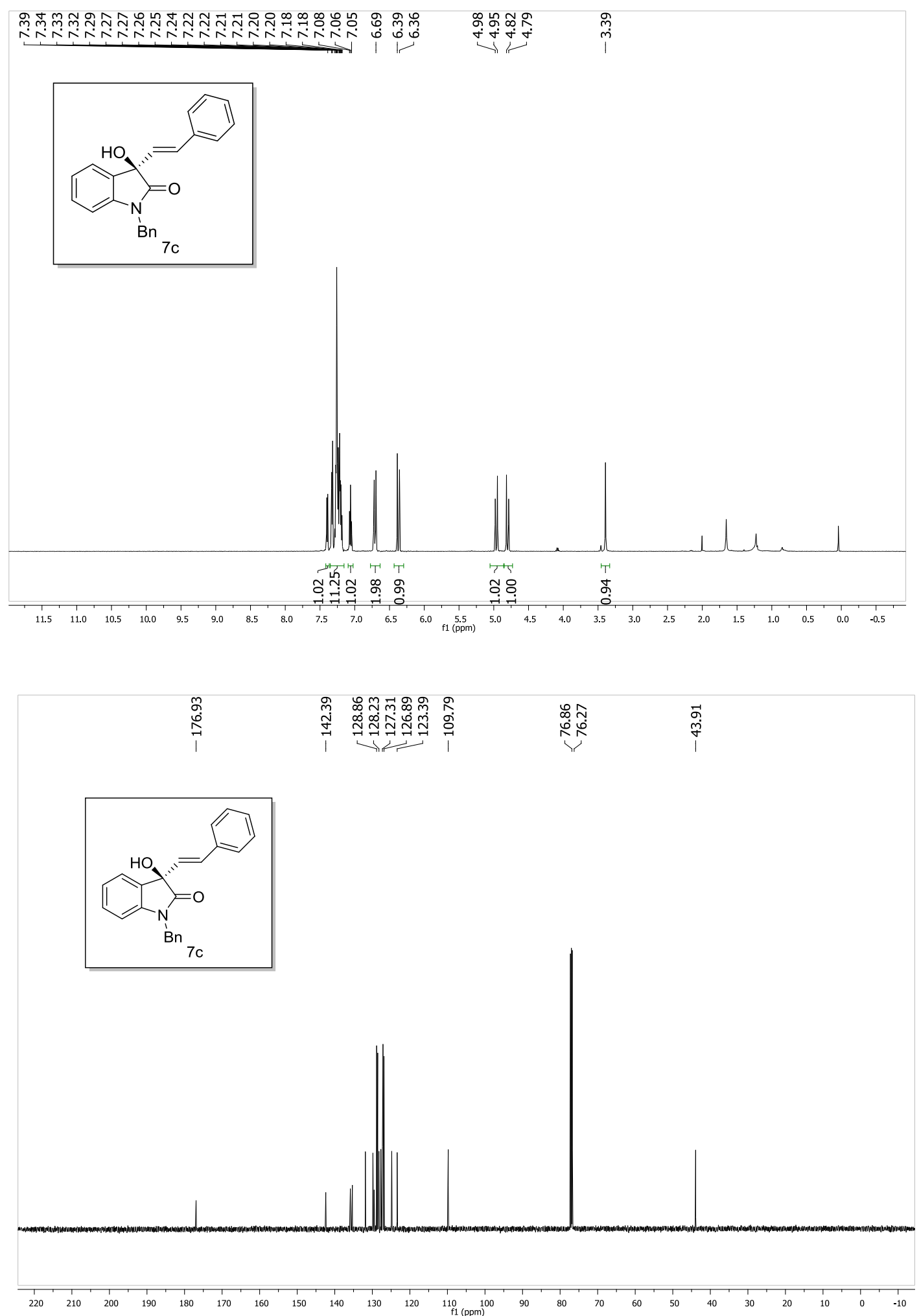

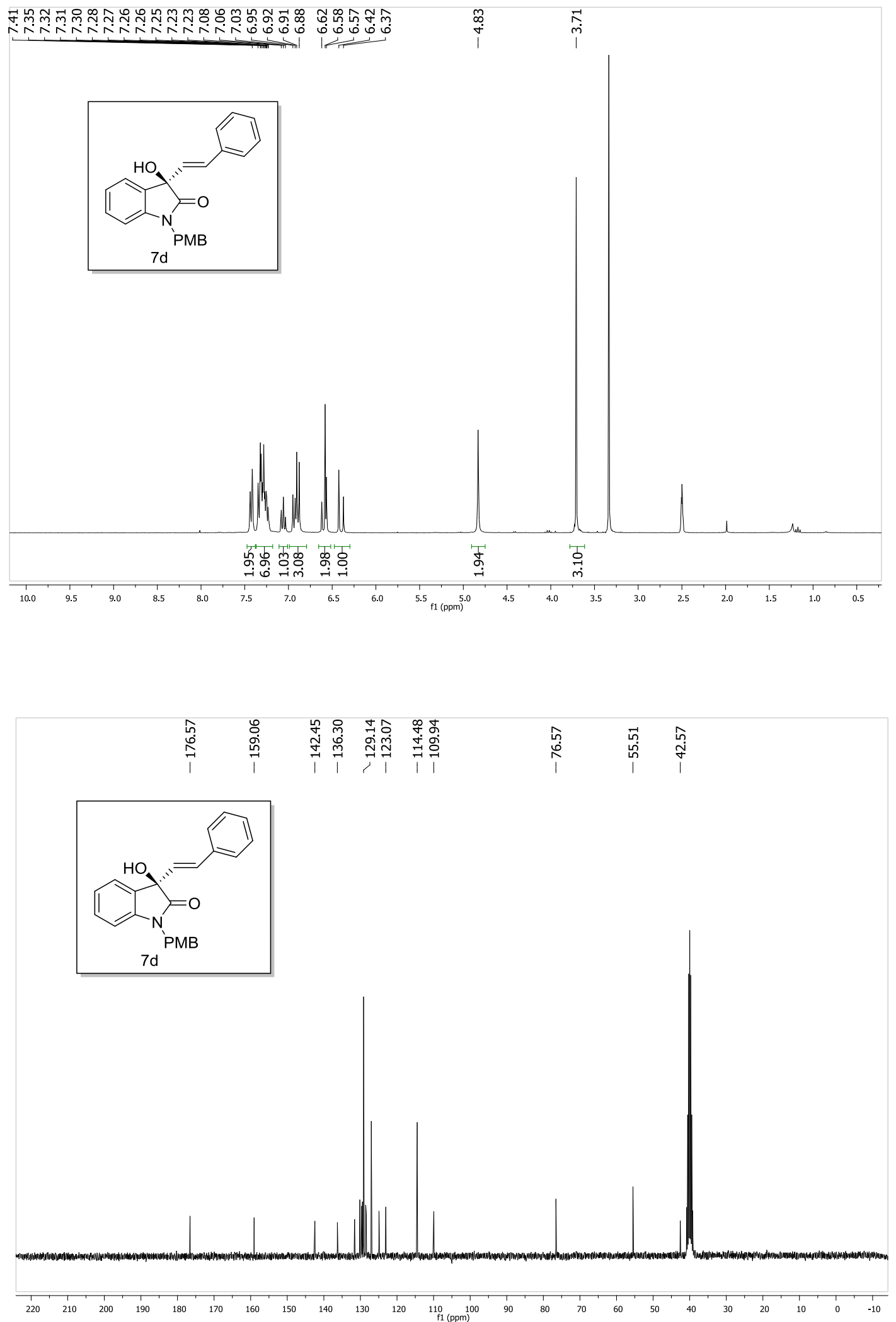

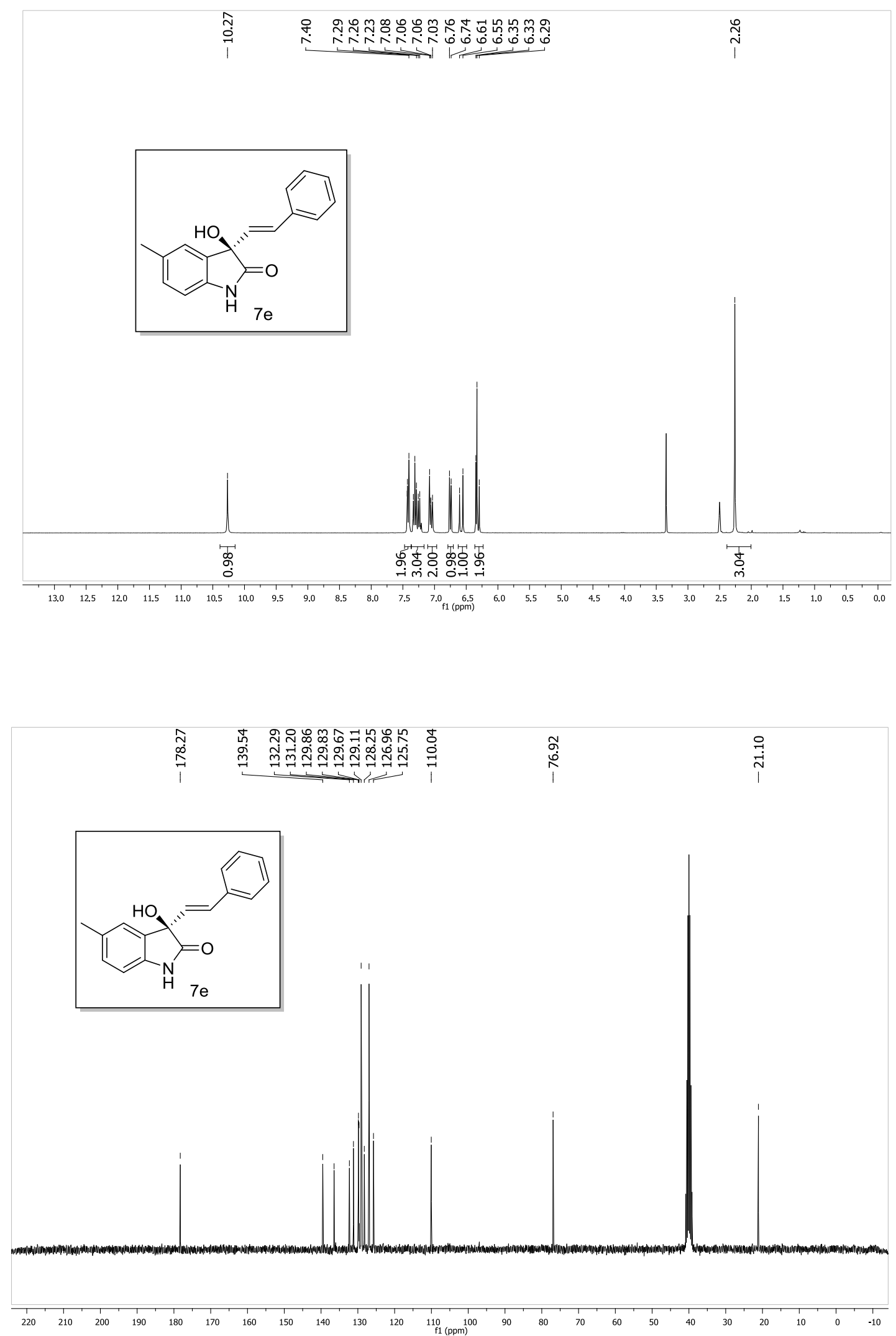

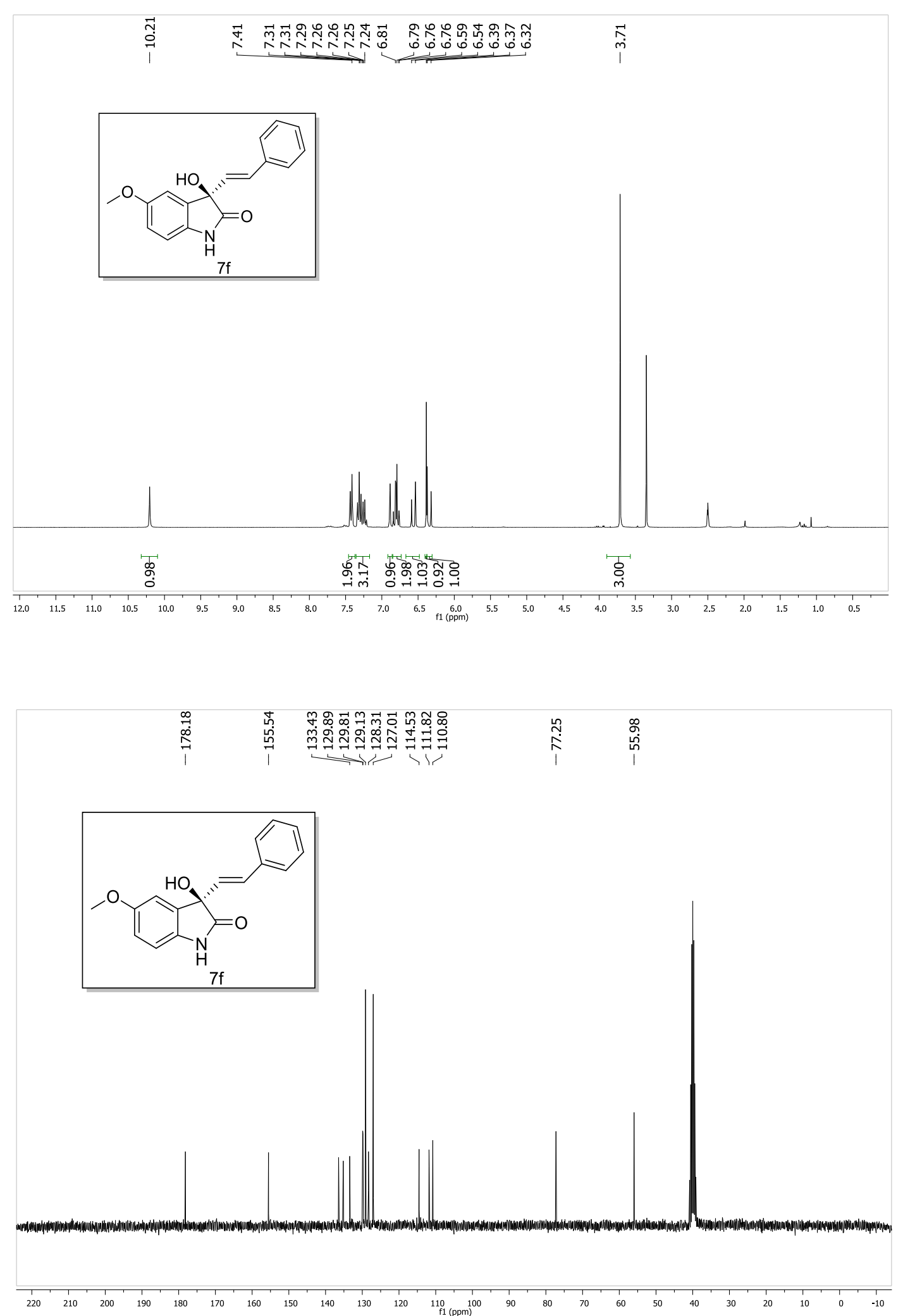

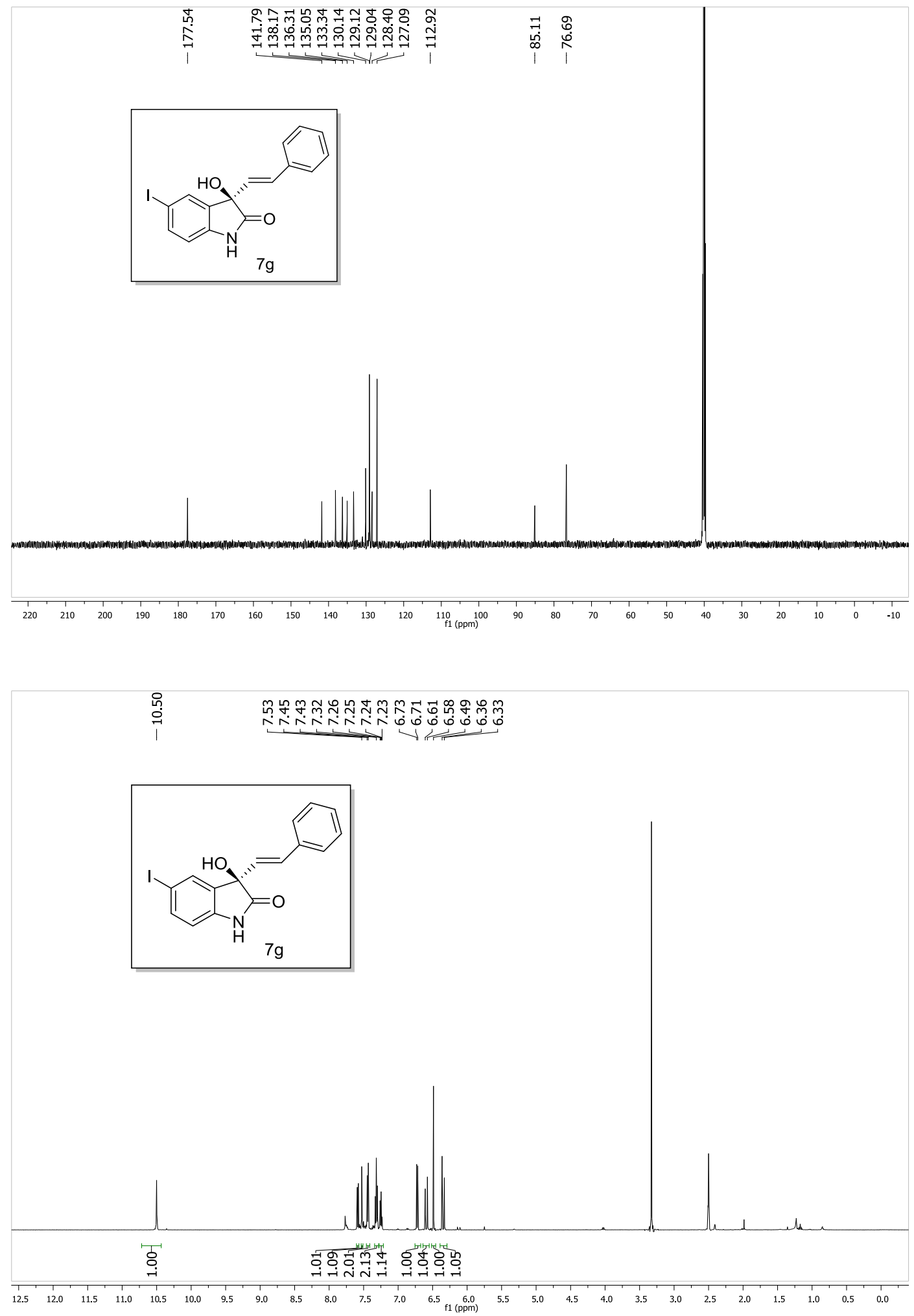

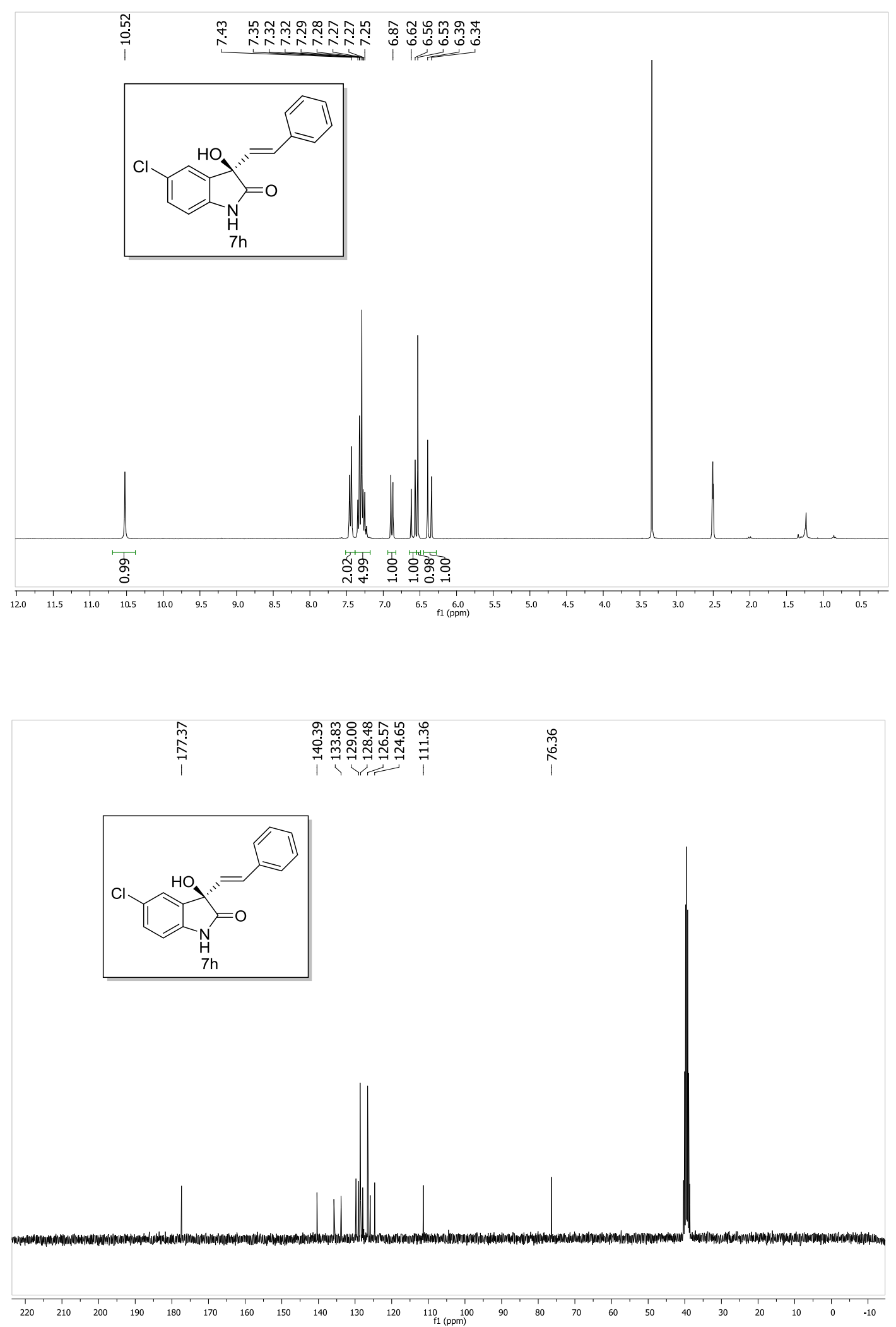

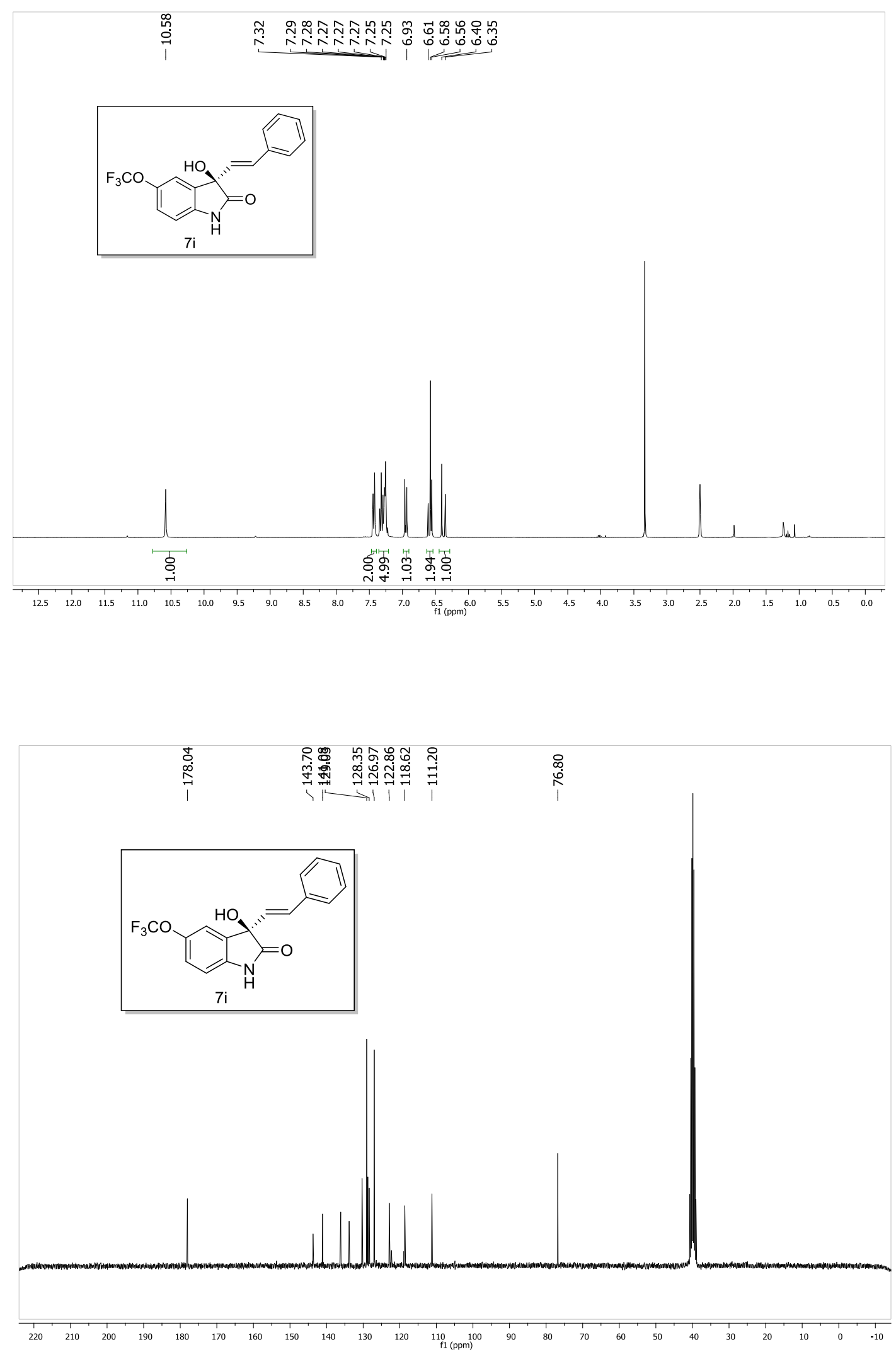


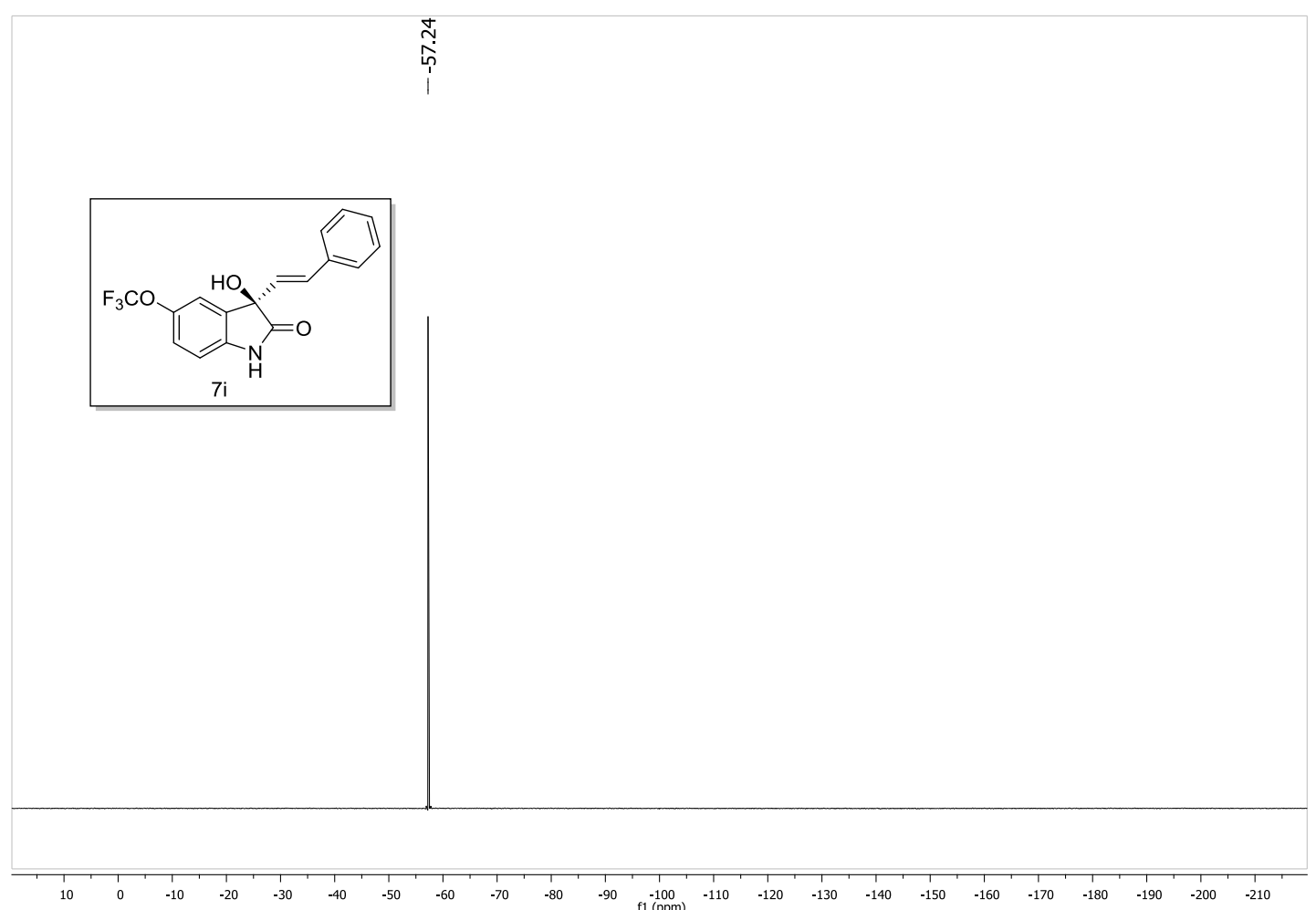



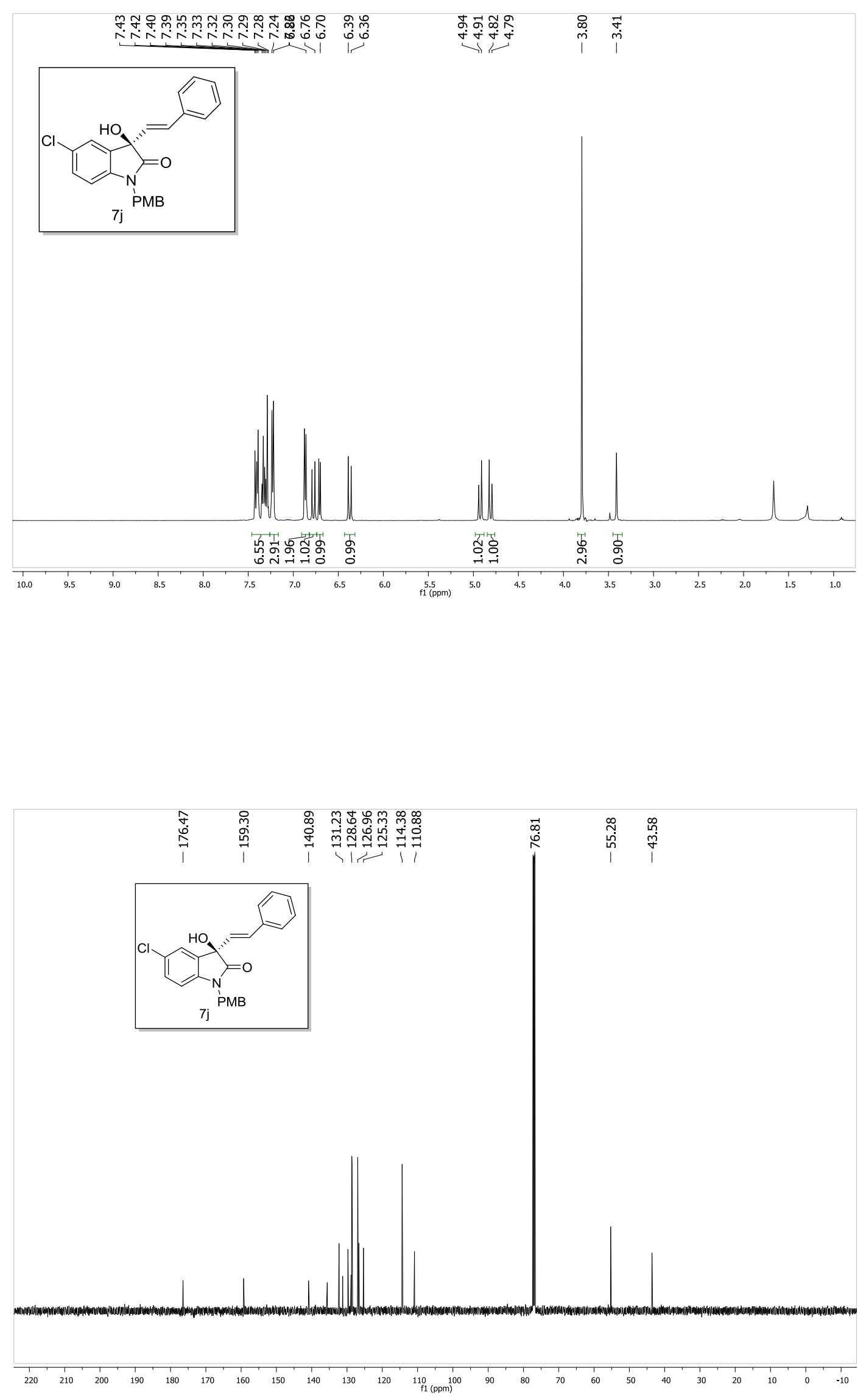

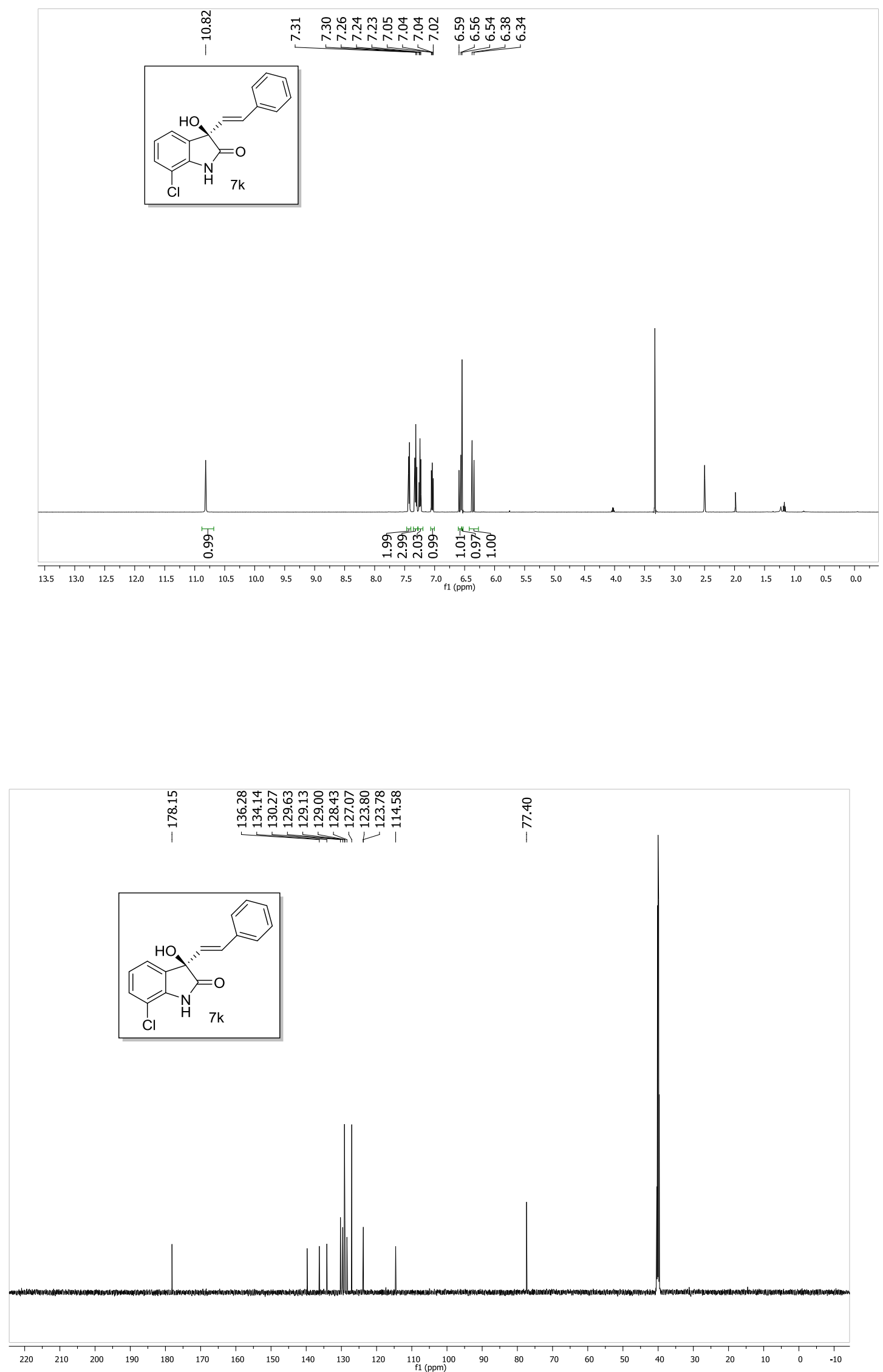

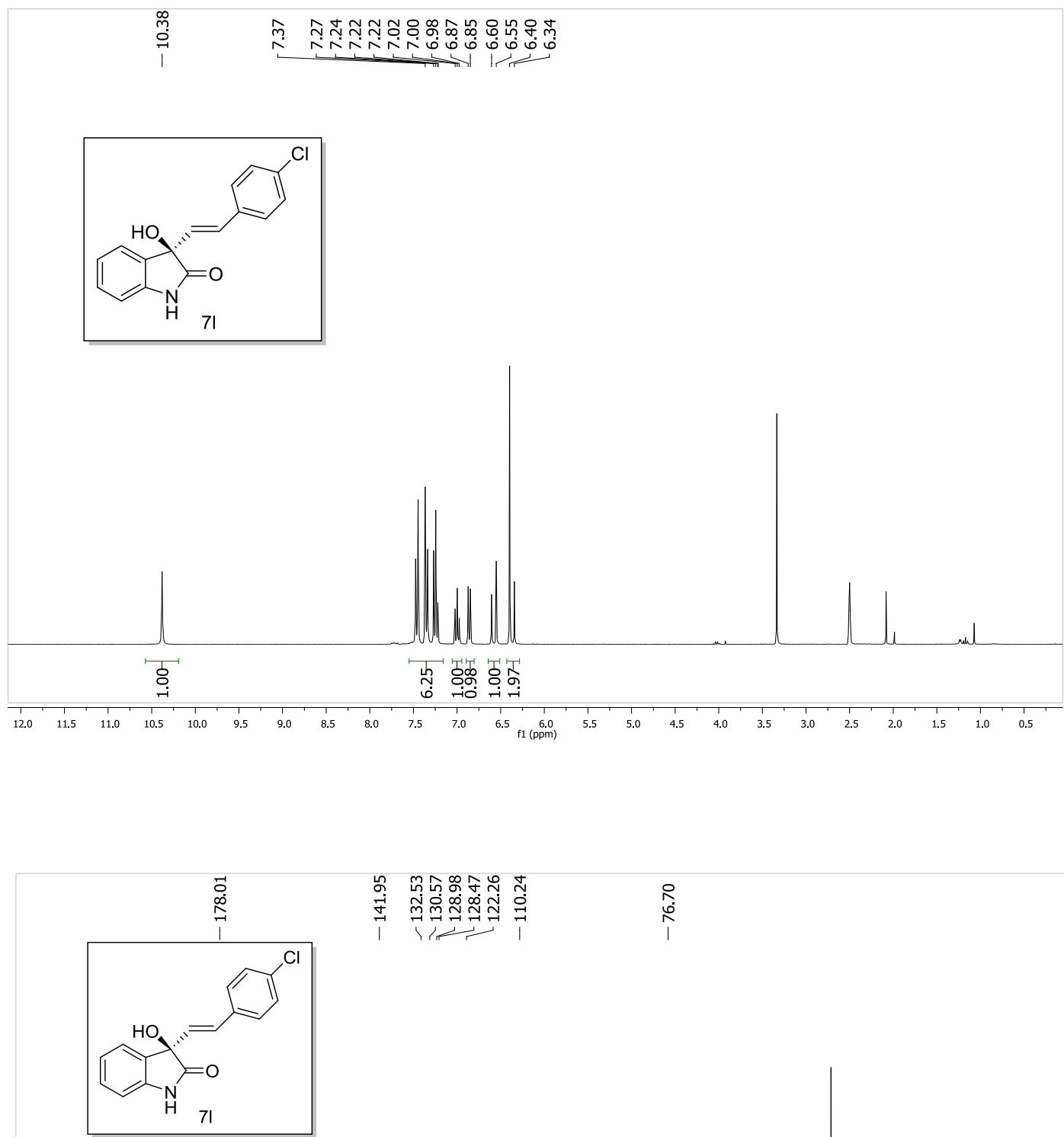

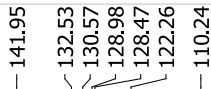

i̊
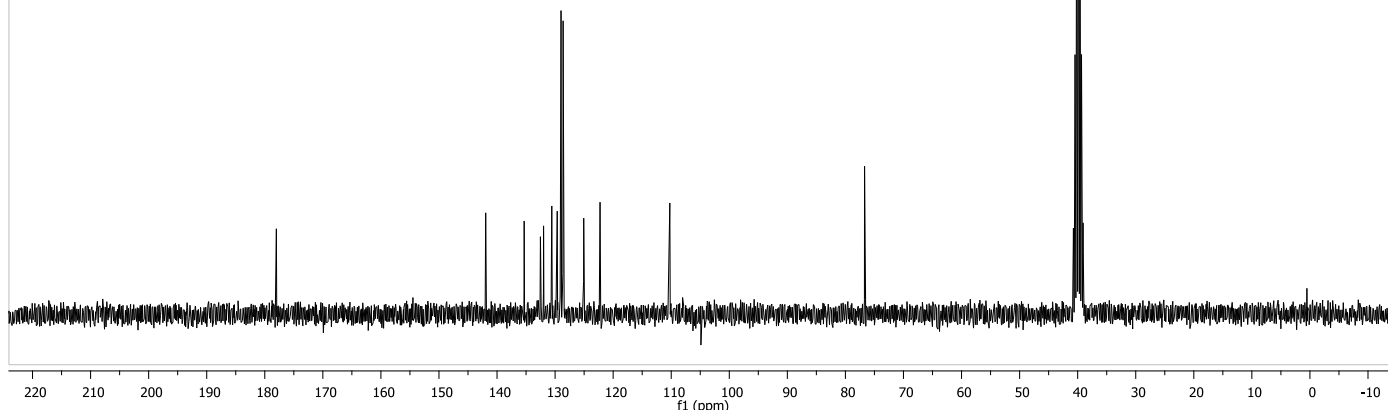

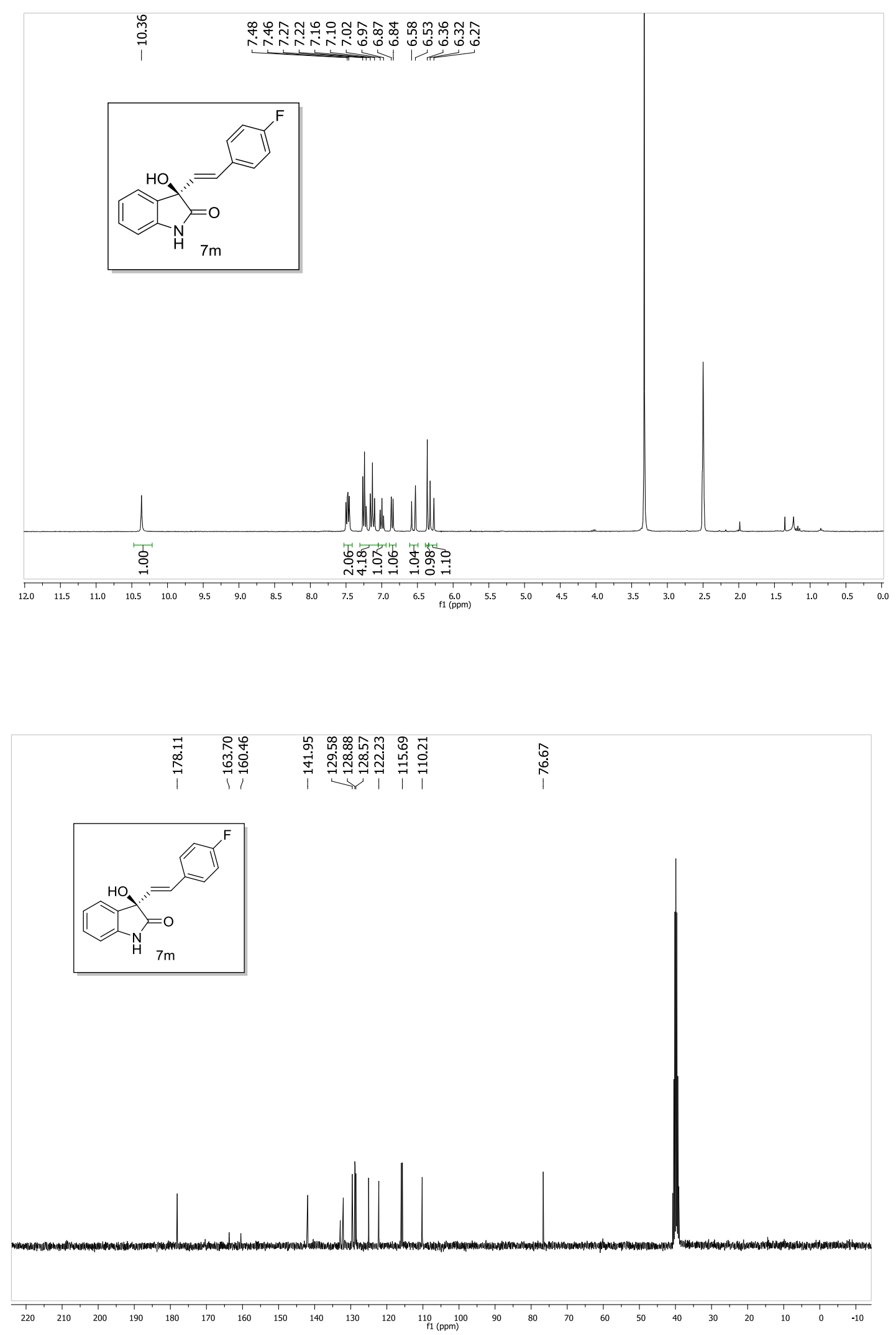


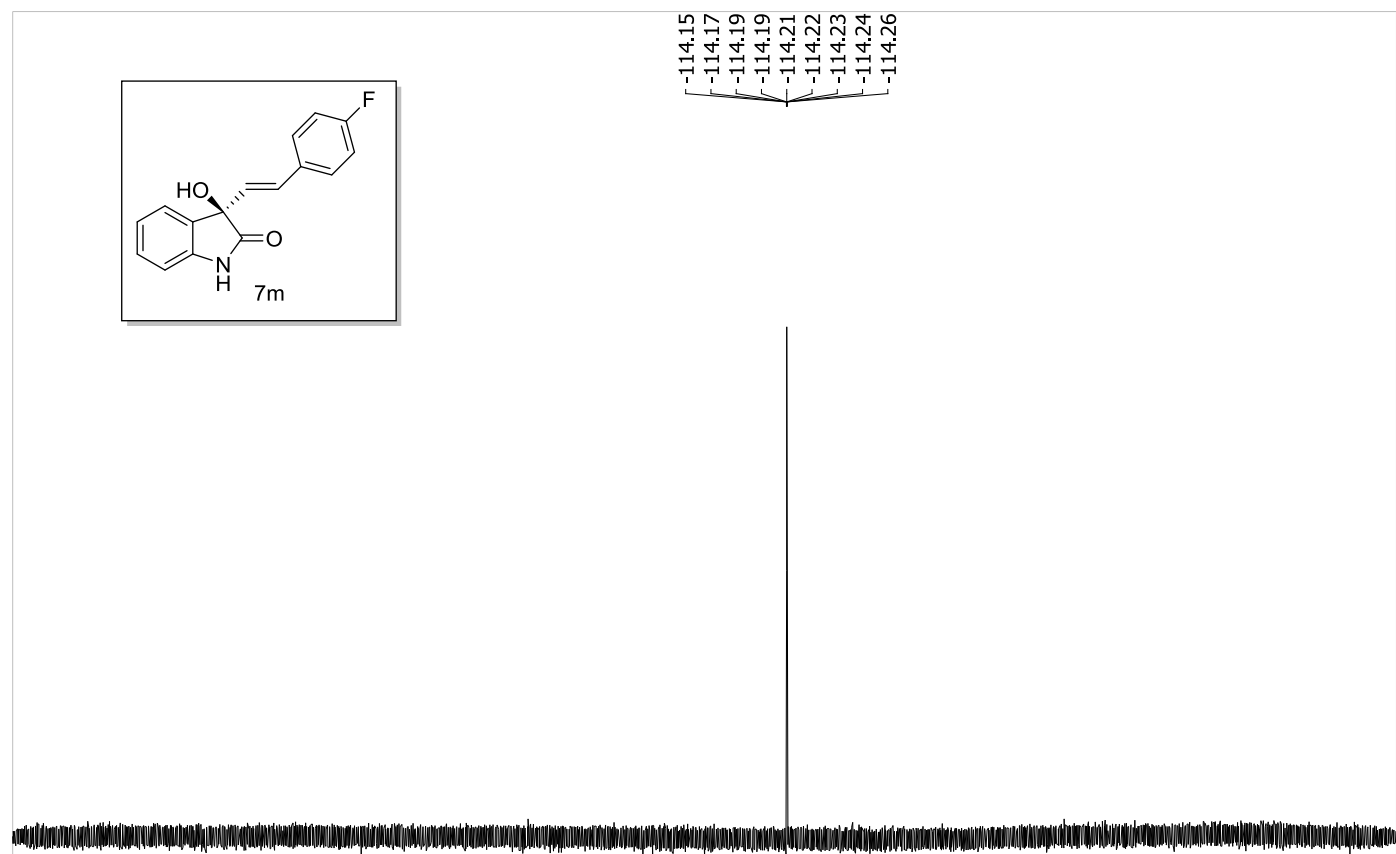

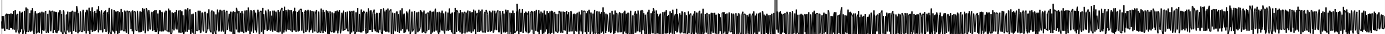
$\begin{array}{lllllllllllllllllllllllllllllllllll}1 & 0 & 0 & -10 & -20 & -30 & -40 & -50 & -60 & -70 & -80 & -90 & -100 & -110 & -120 & -130 & -140 & -150 & -160 & -170 & -180 & -190 & -200 & -210 & 1\end{array}$ 

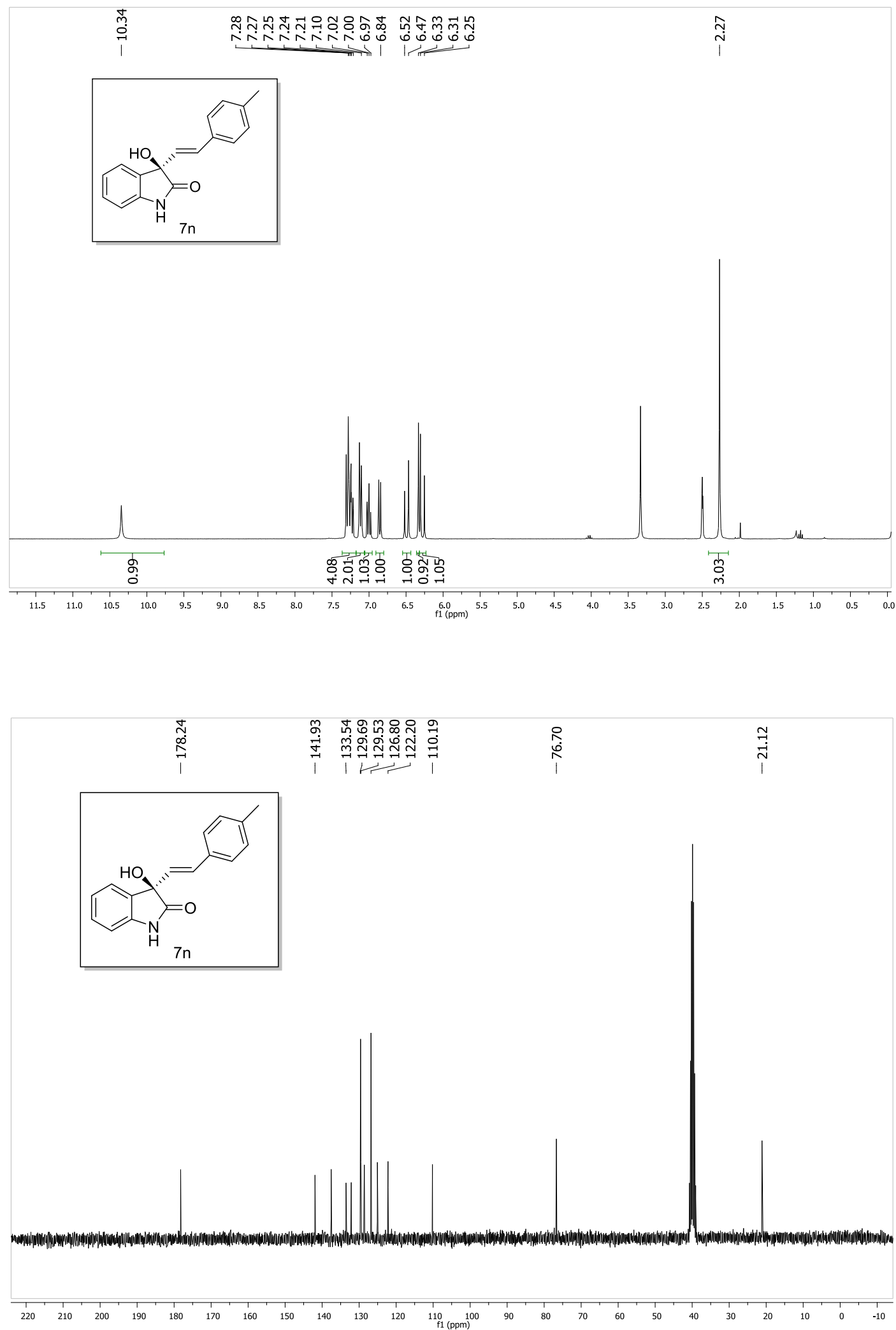

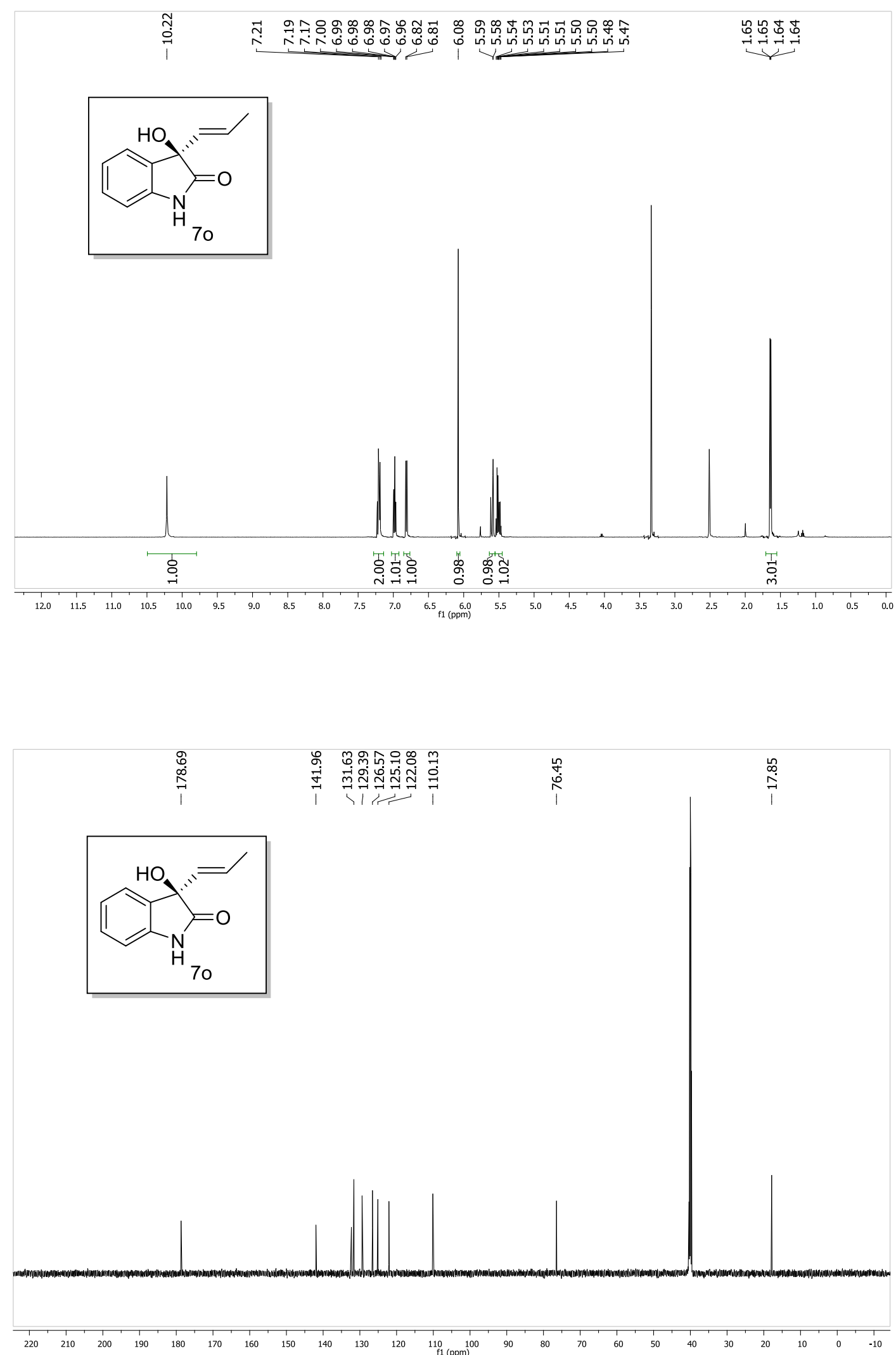

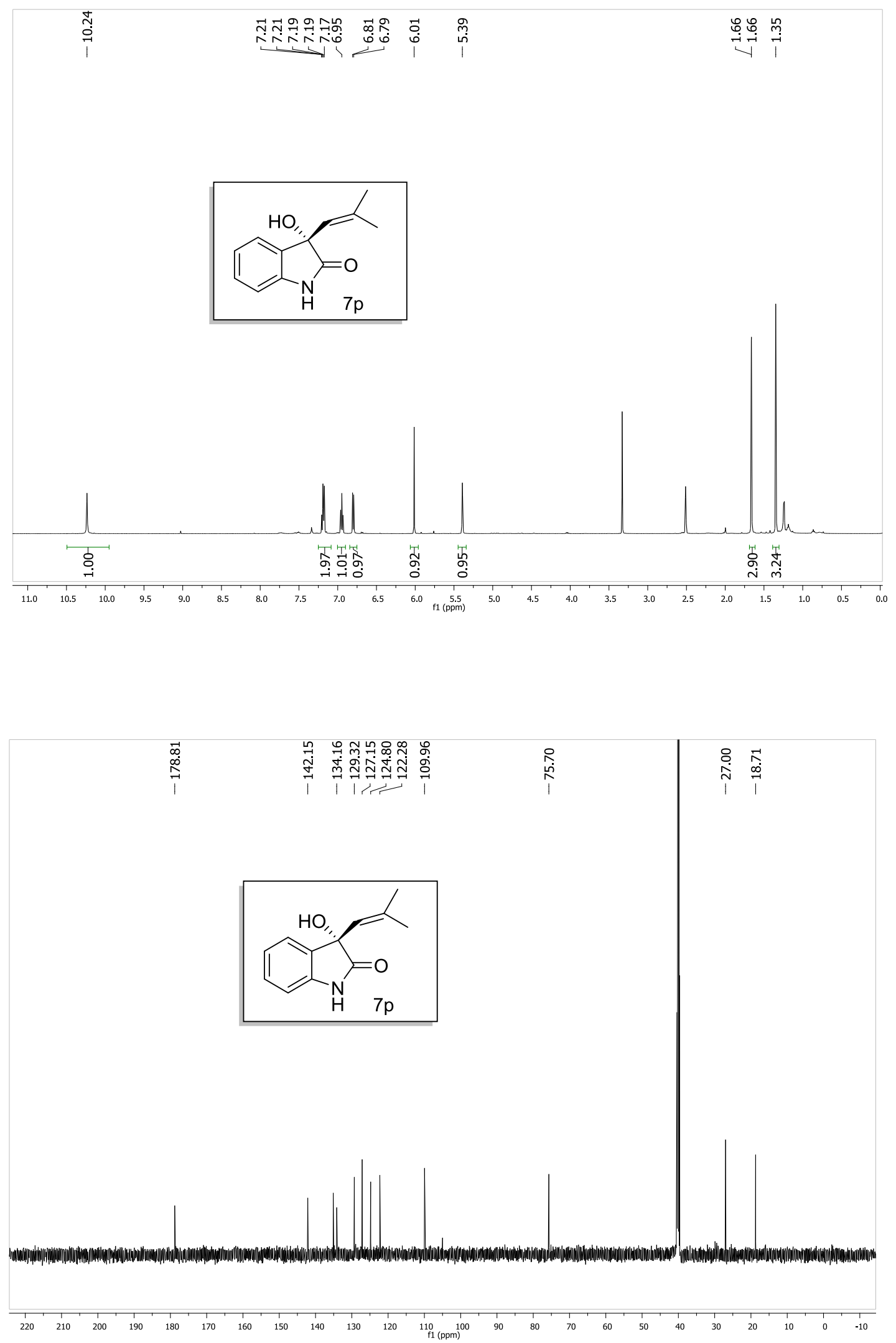

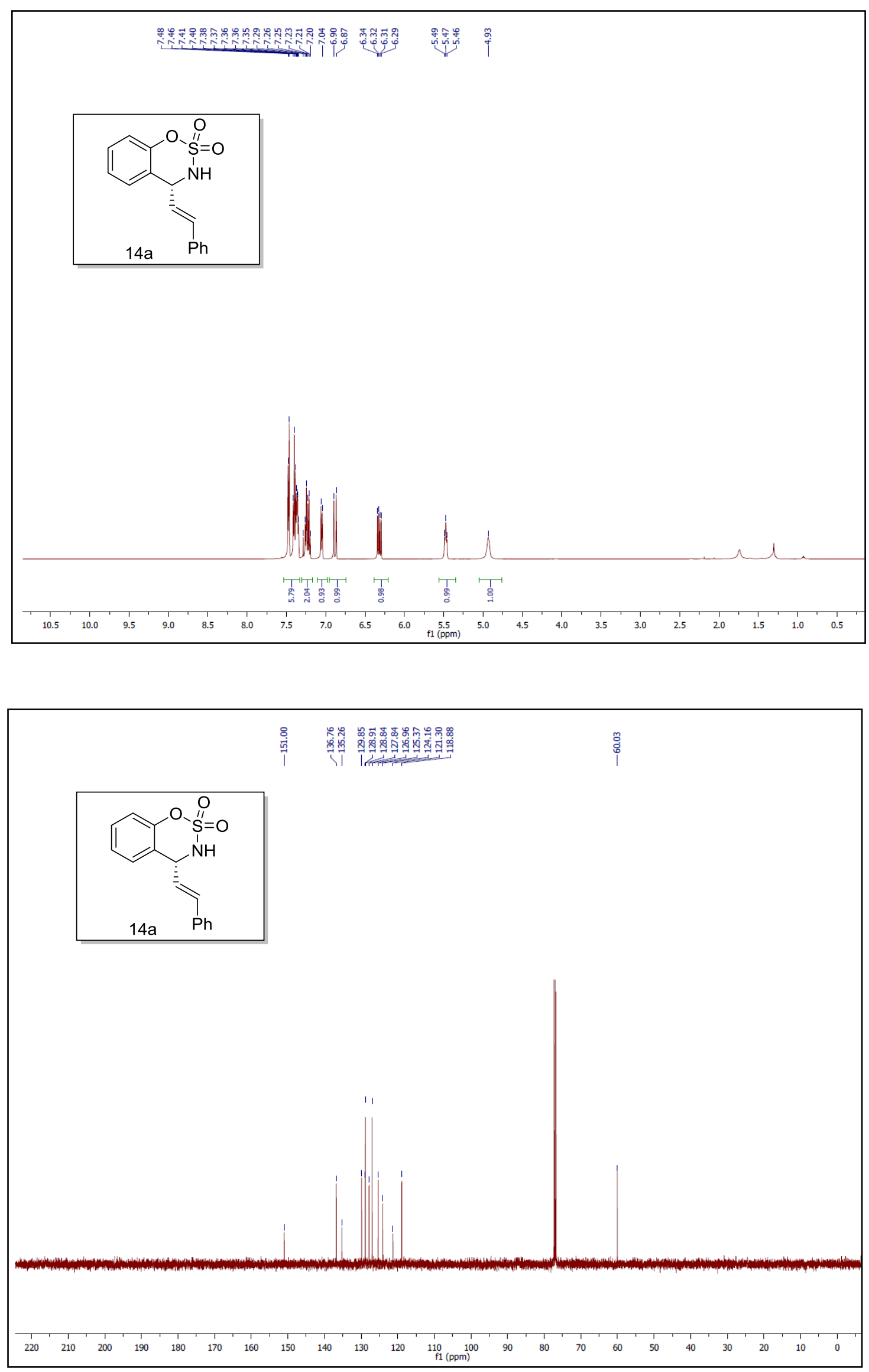

86 

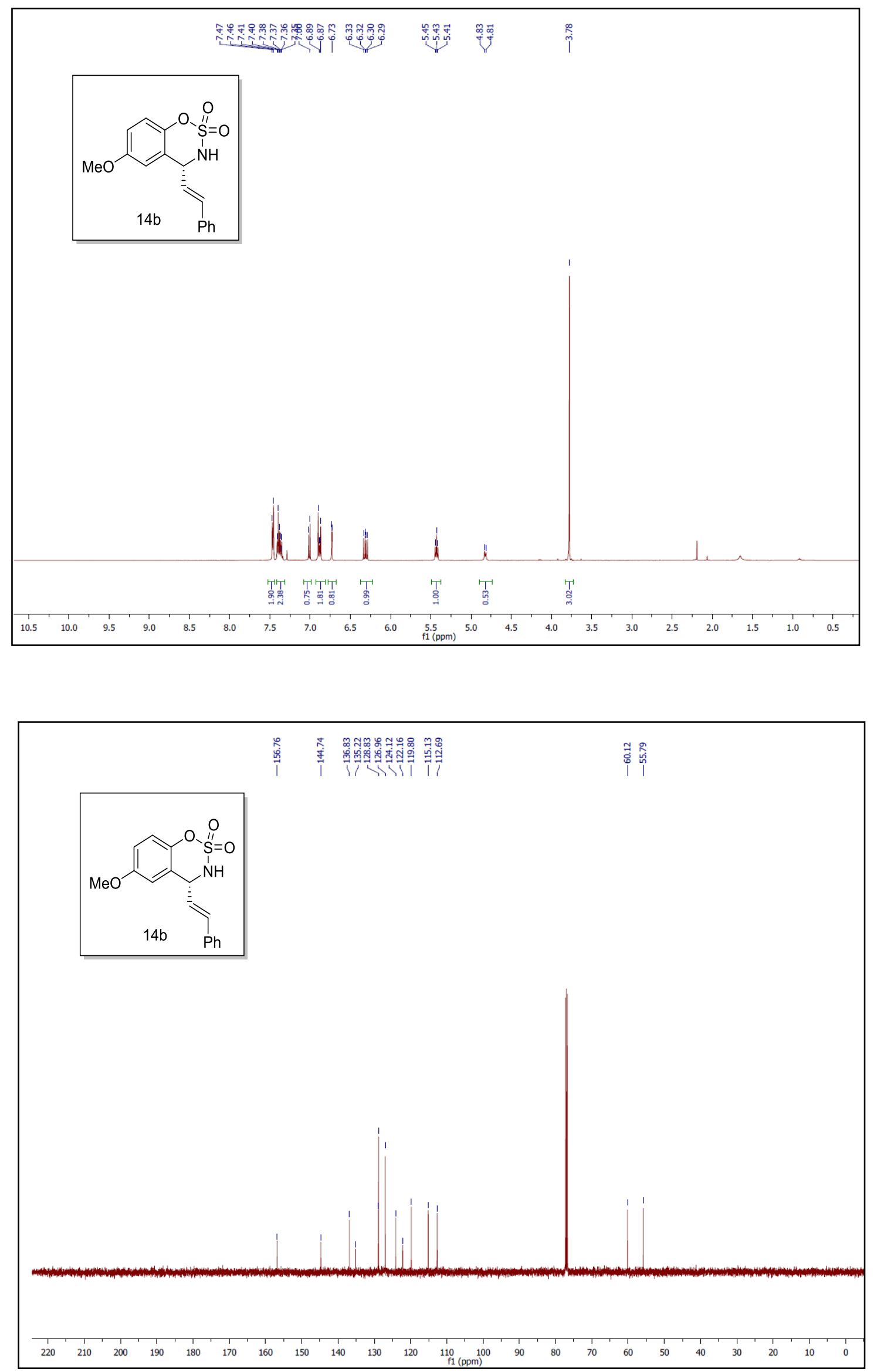

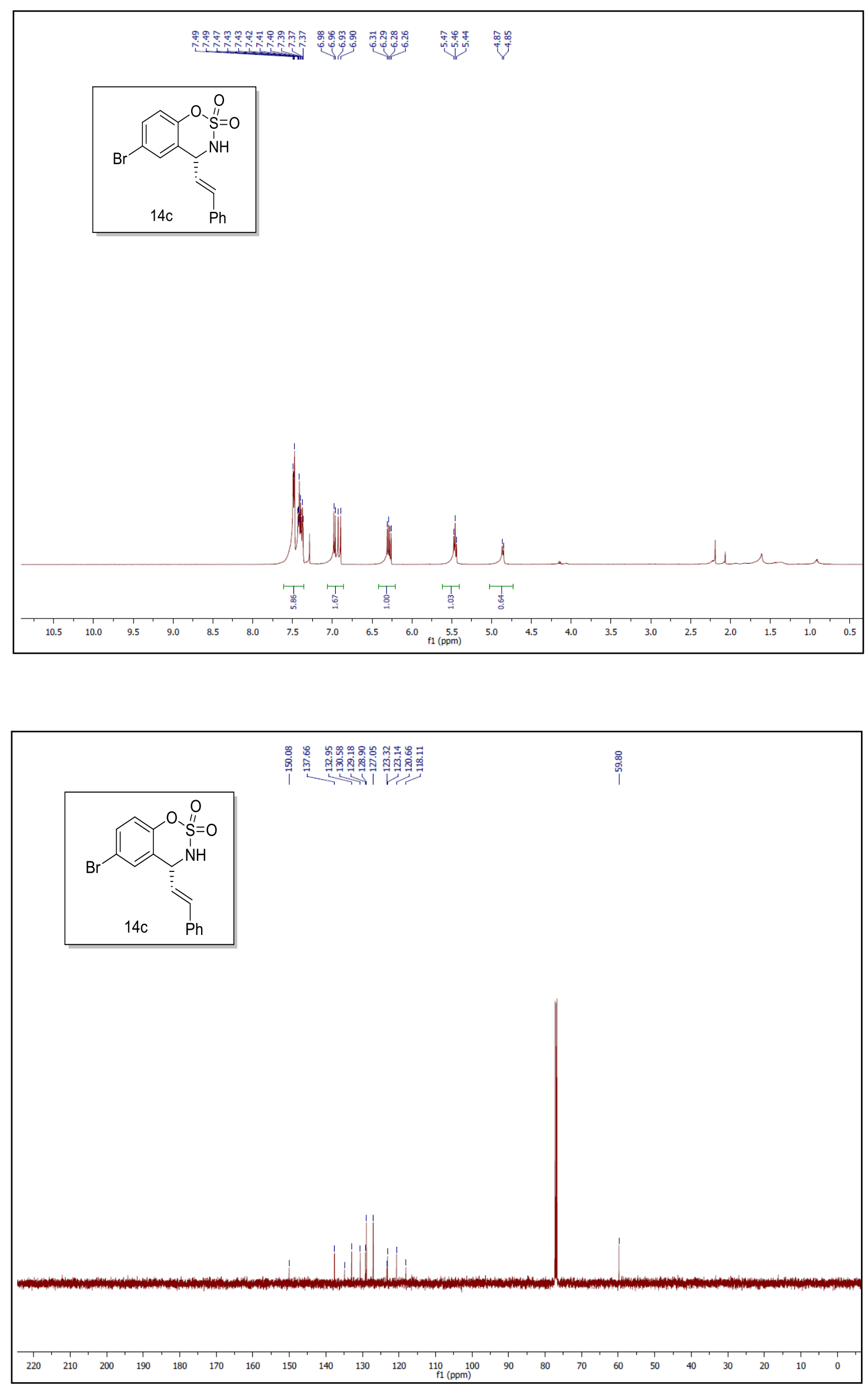

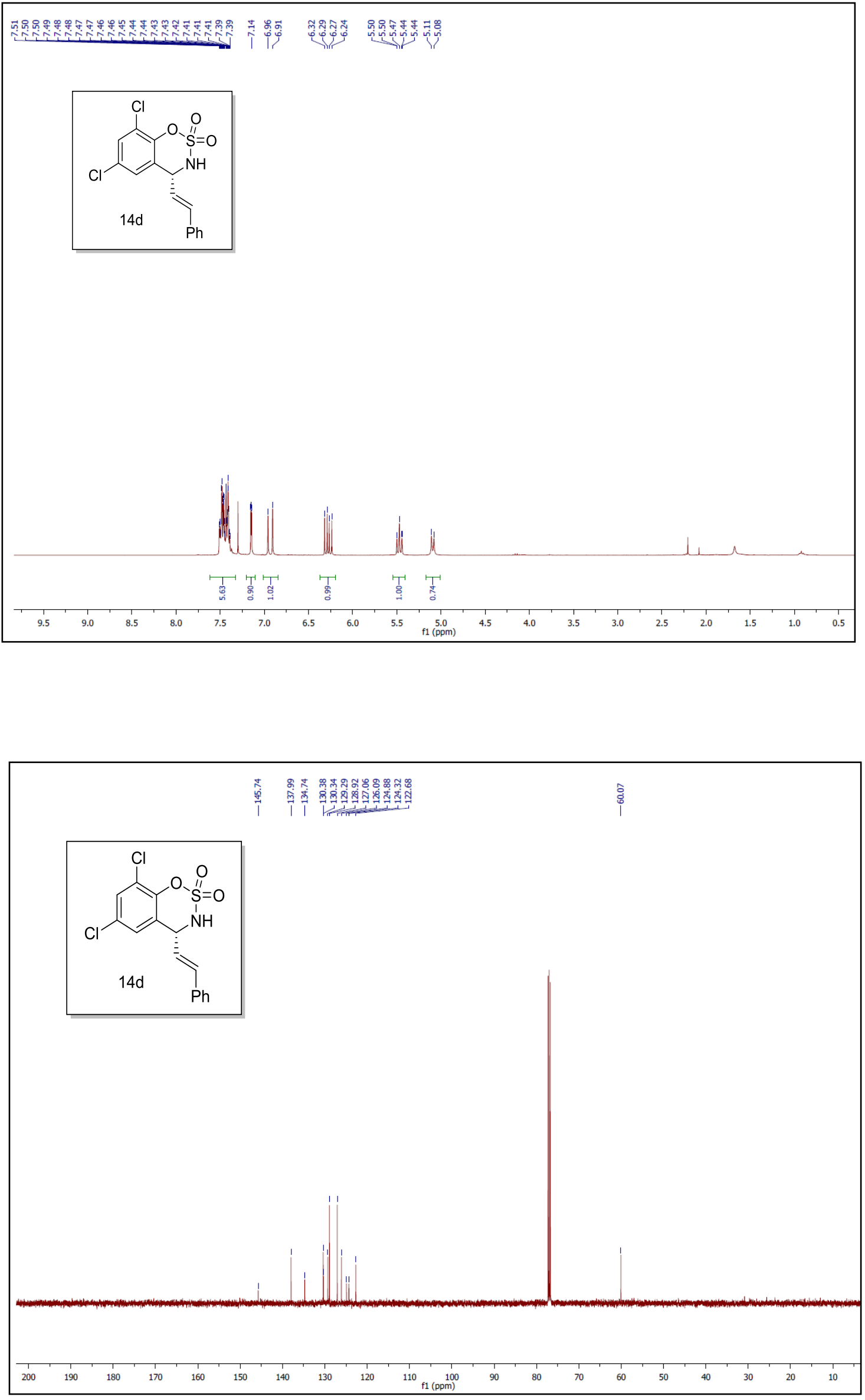

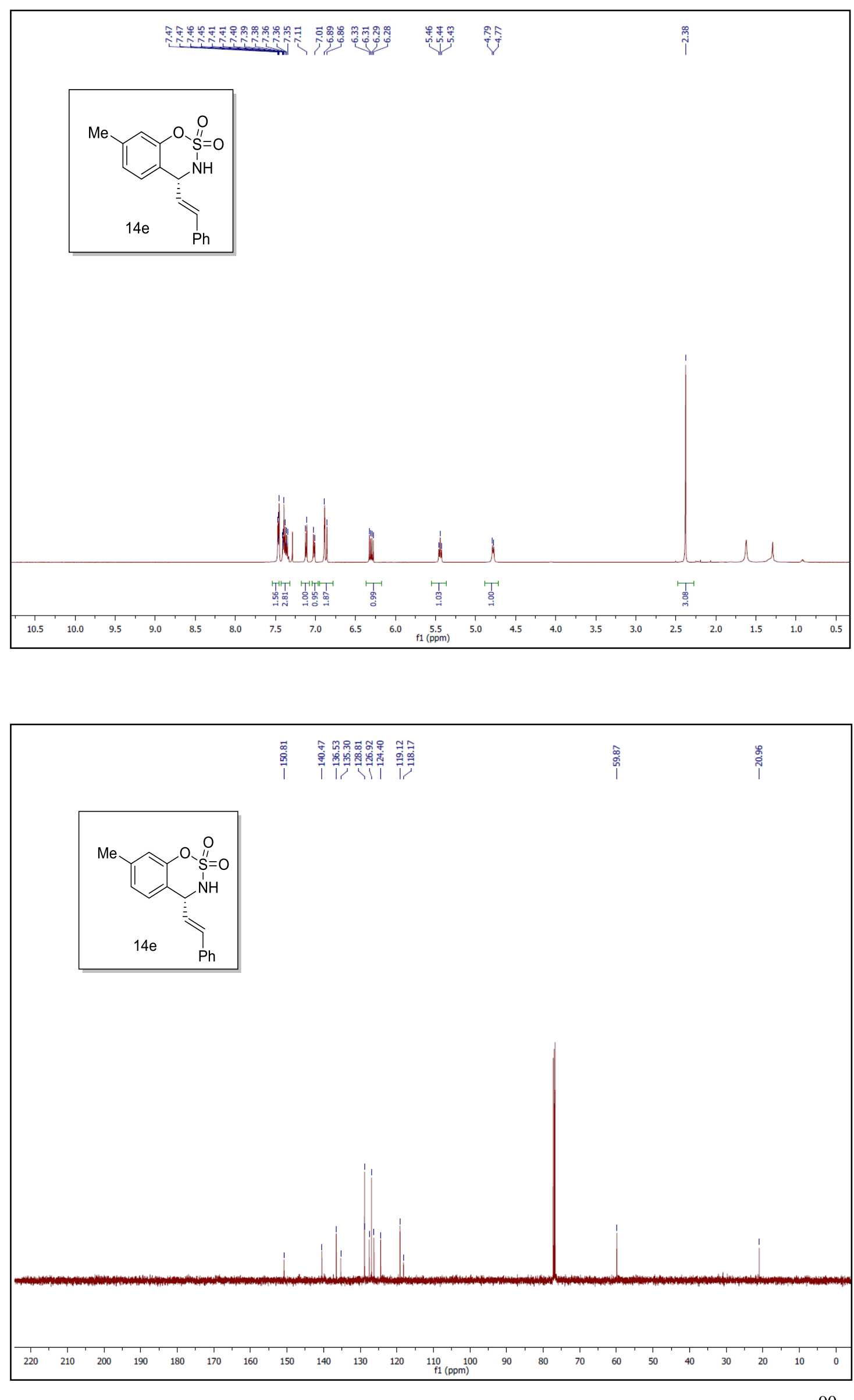

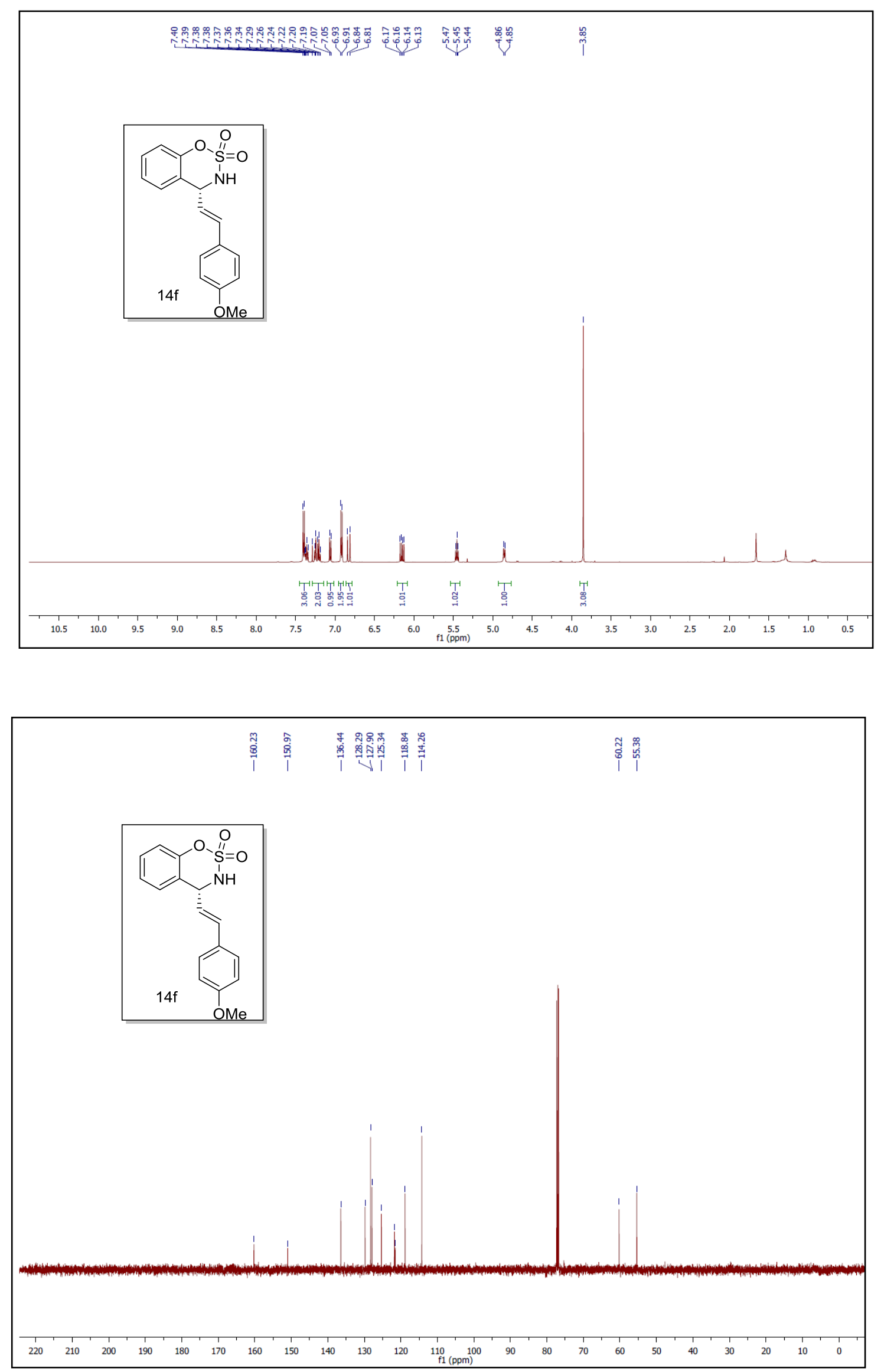

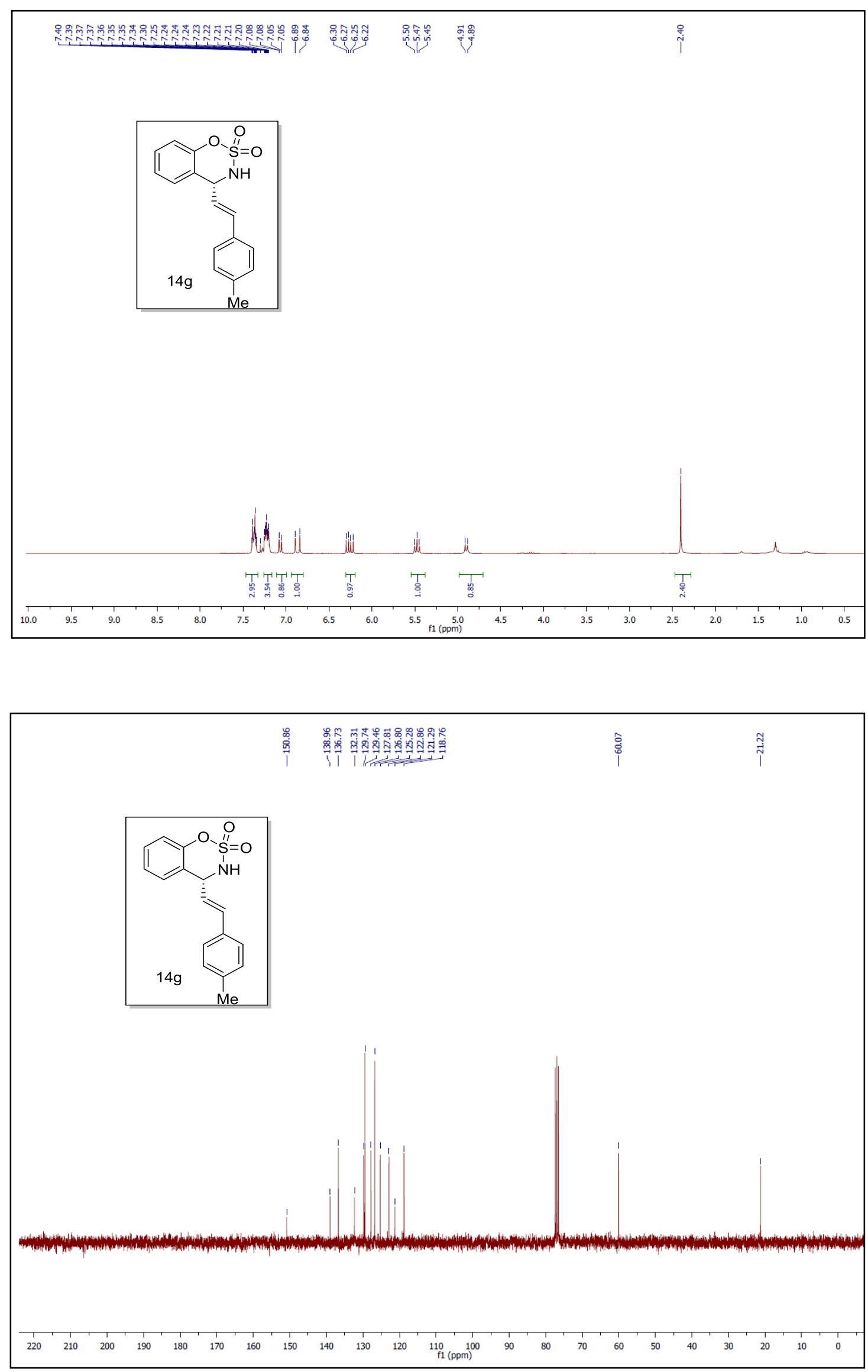

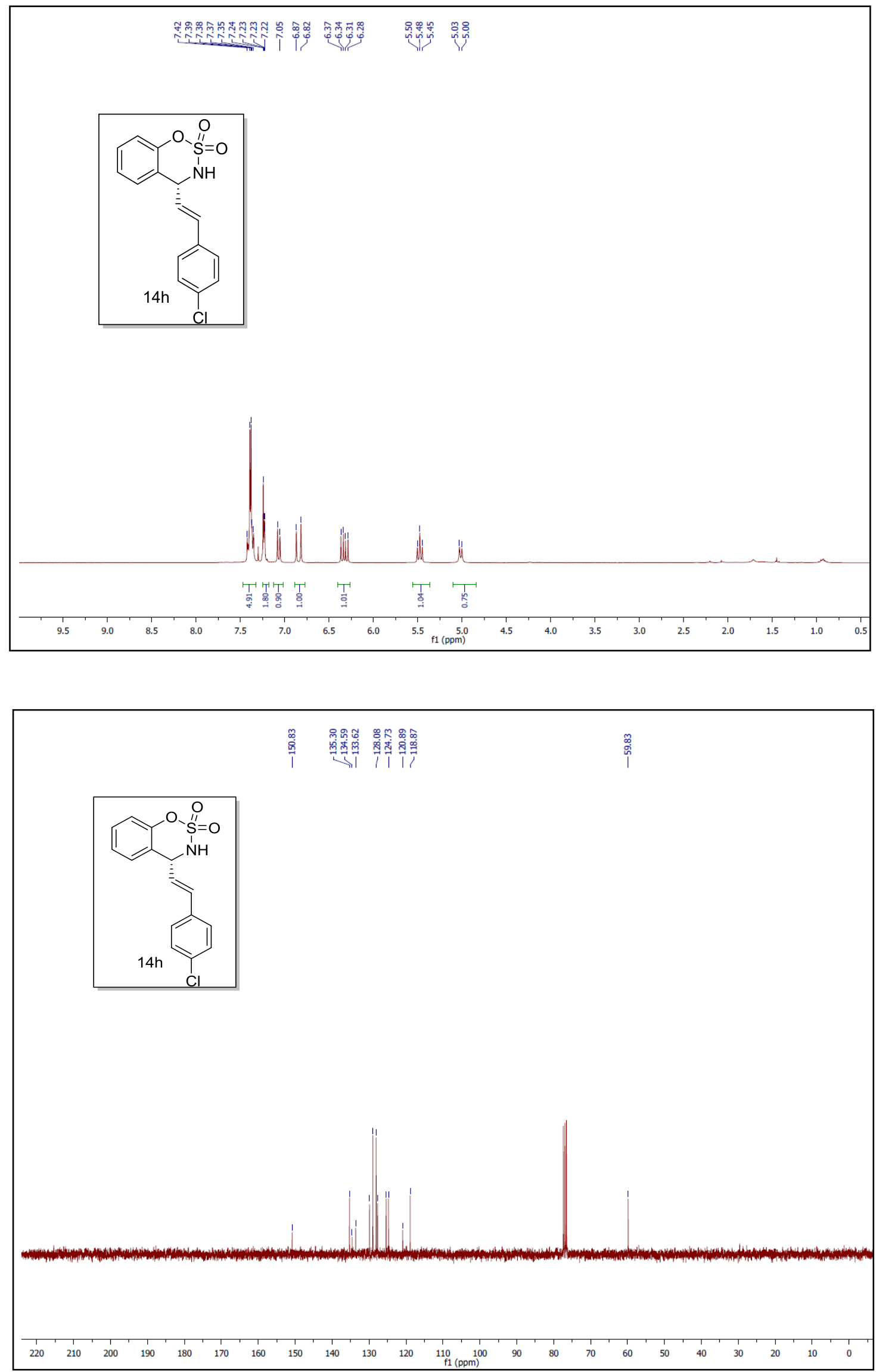

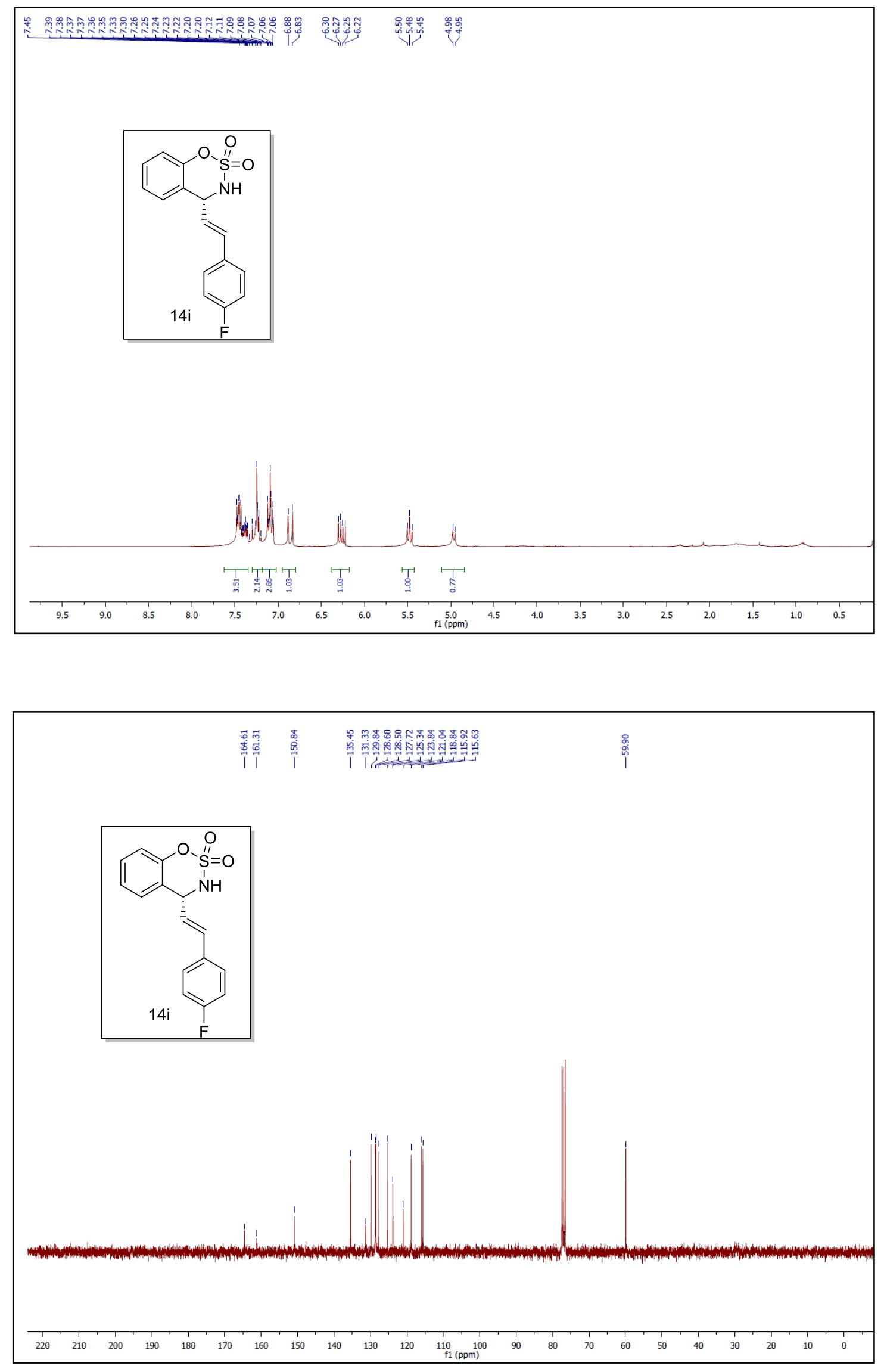


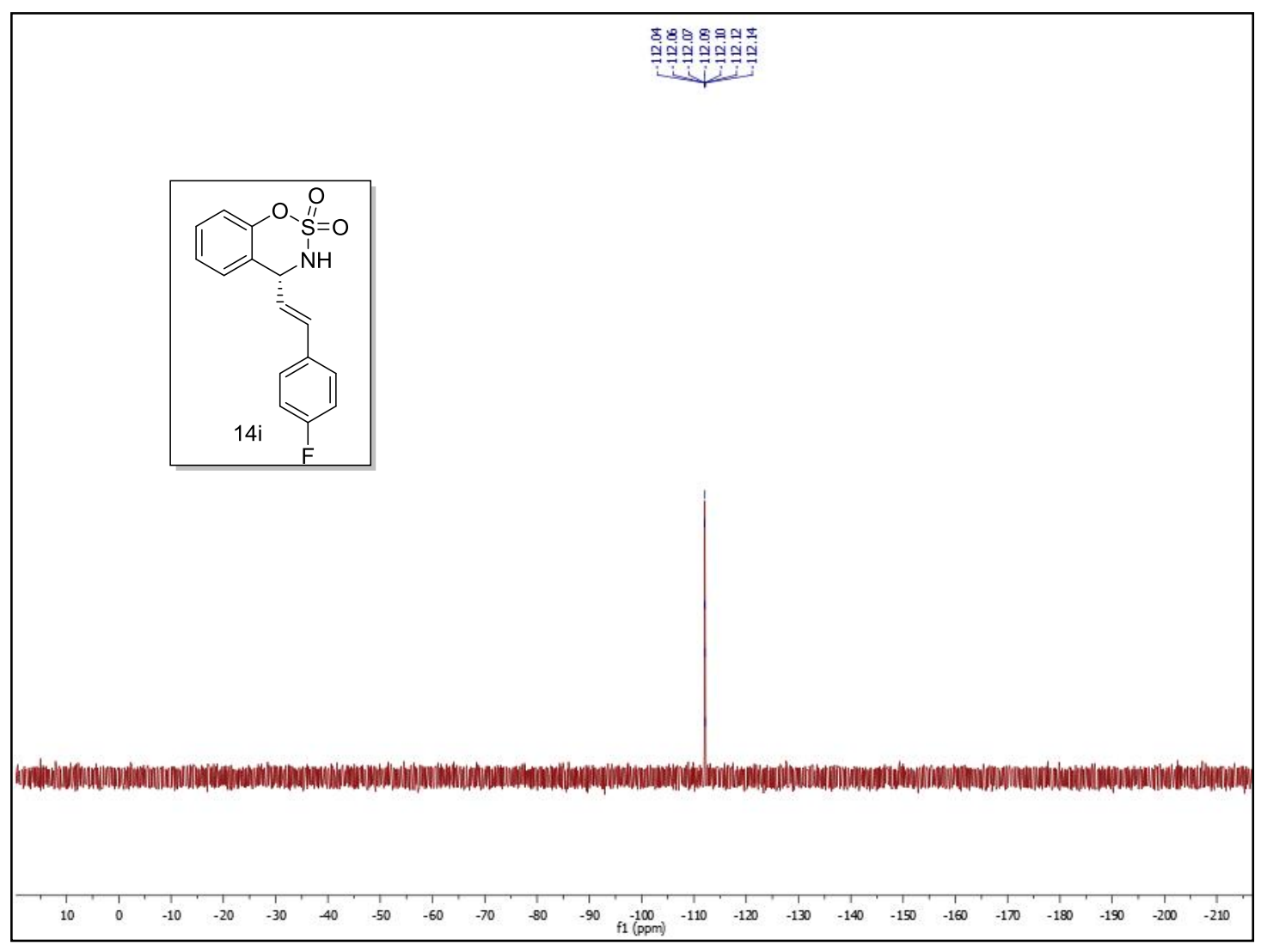



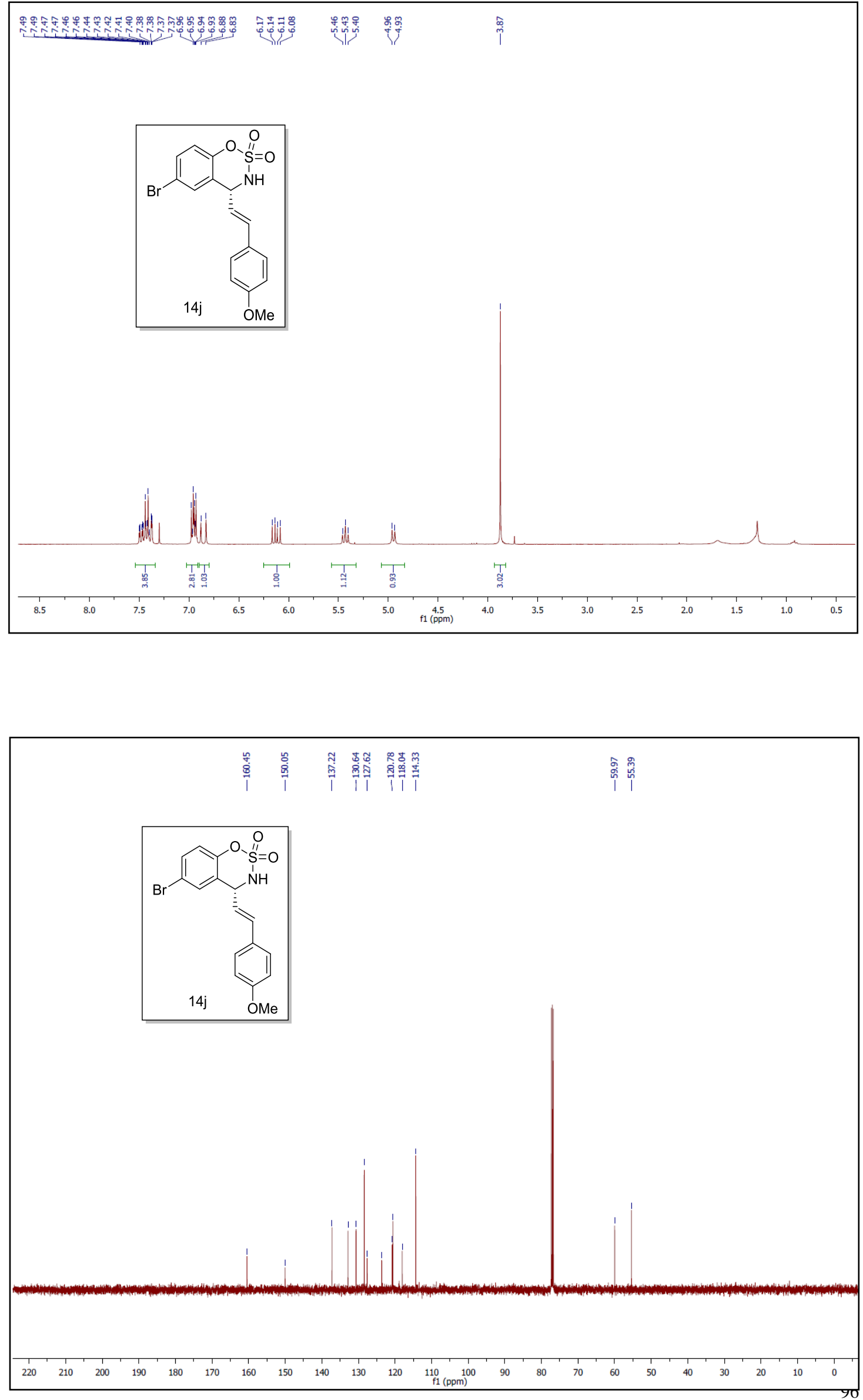

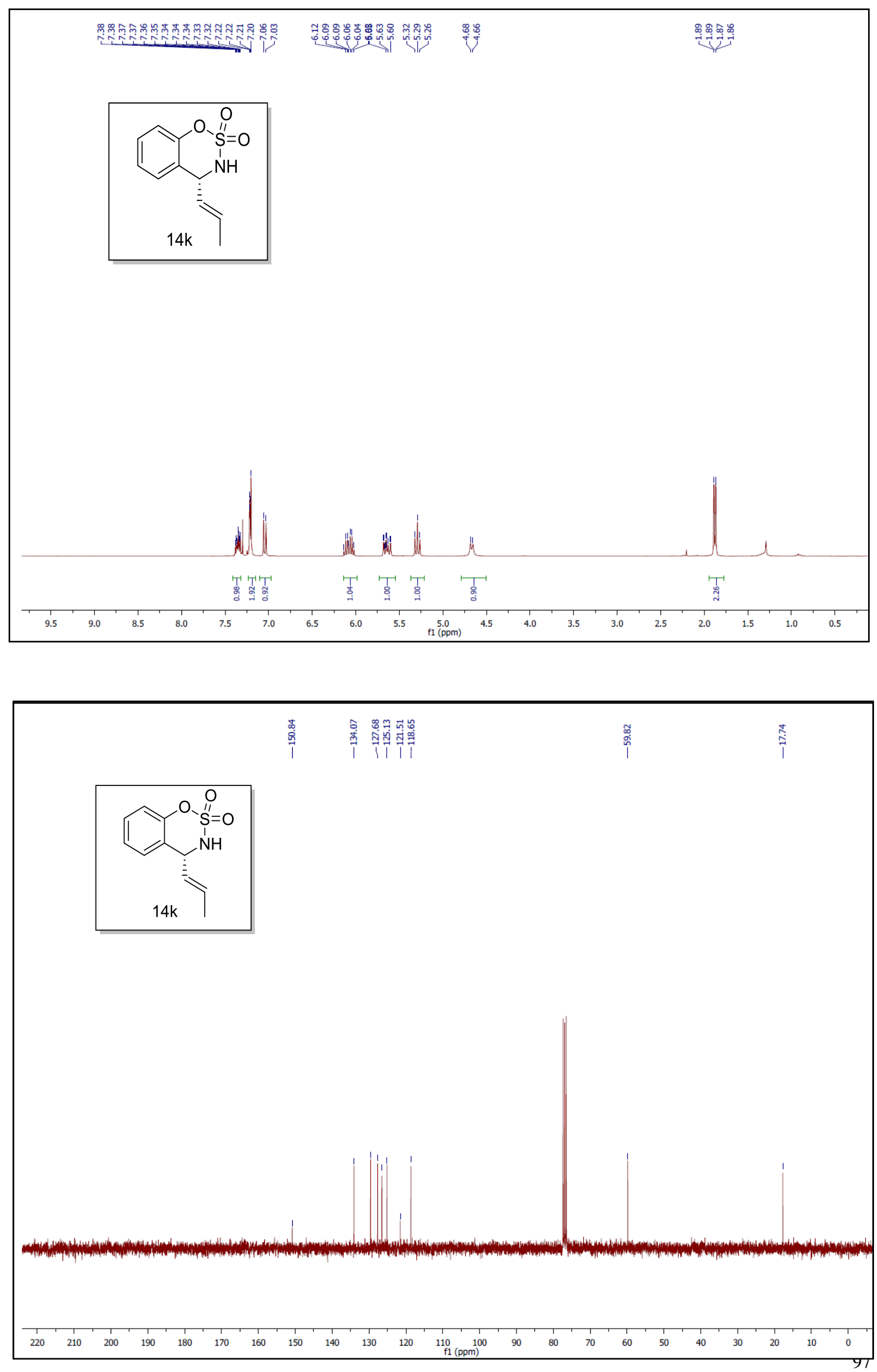

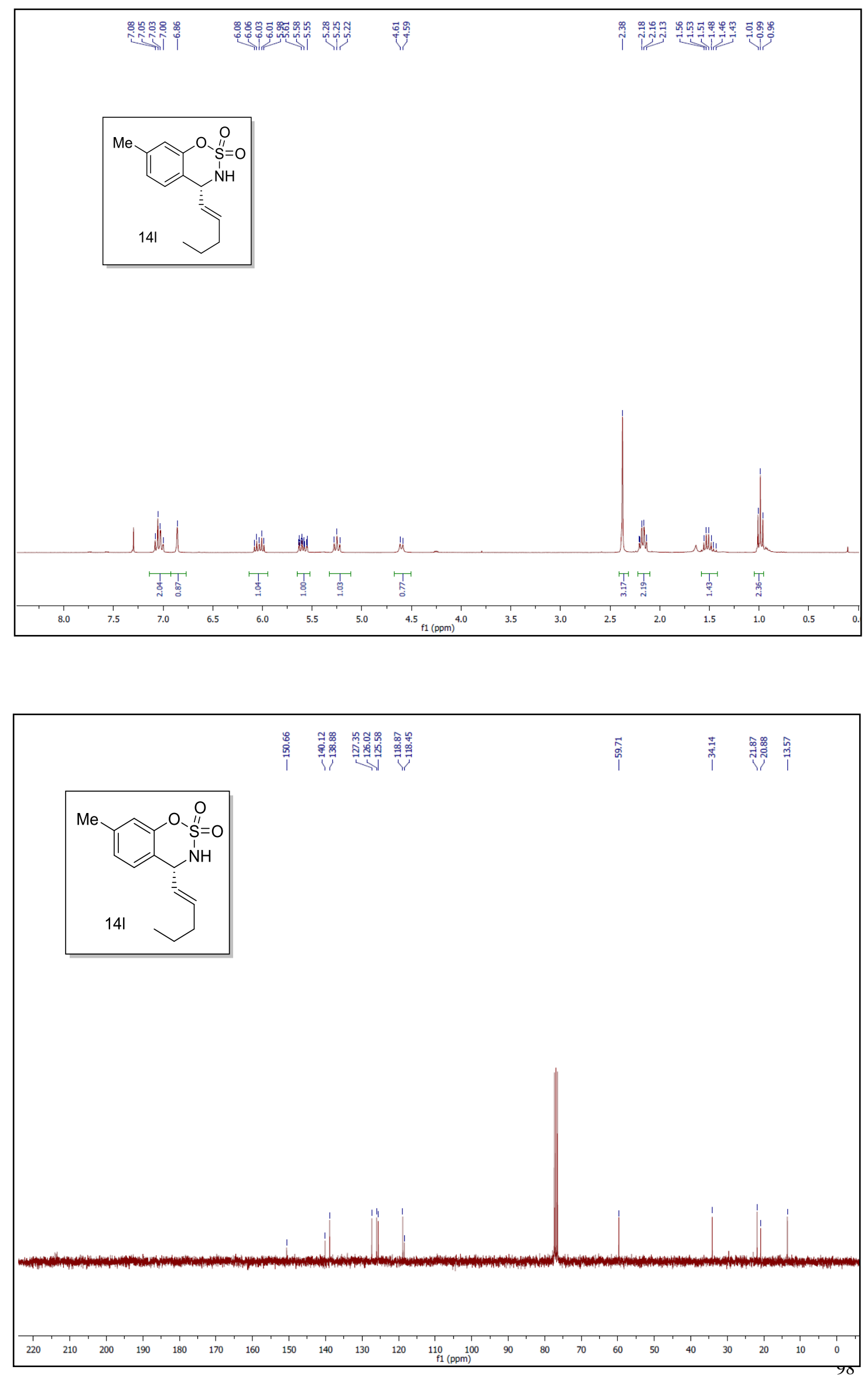

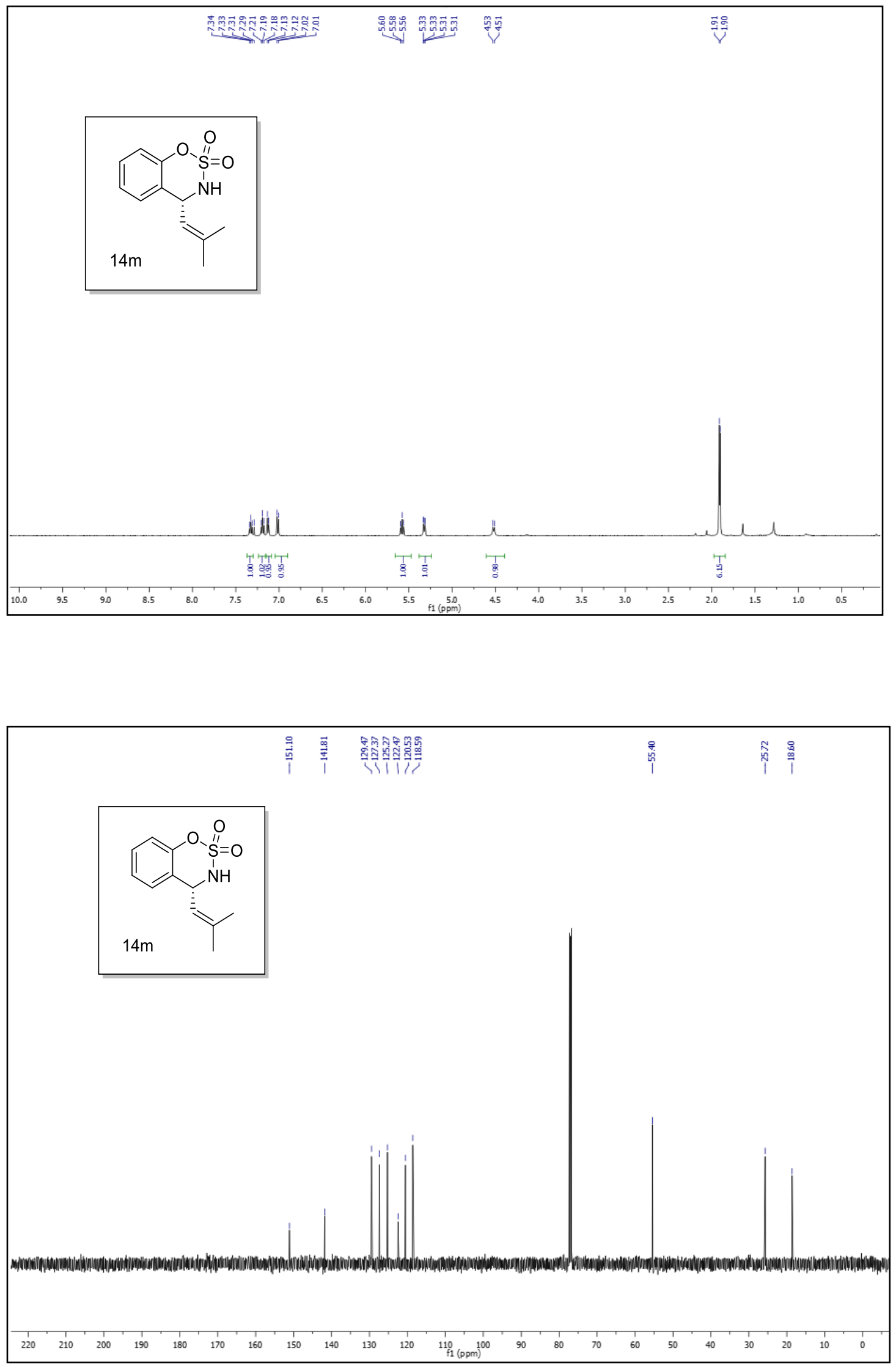
NMR spectra of the derivatives:
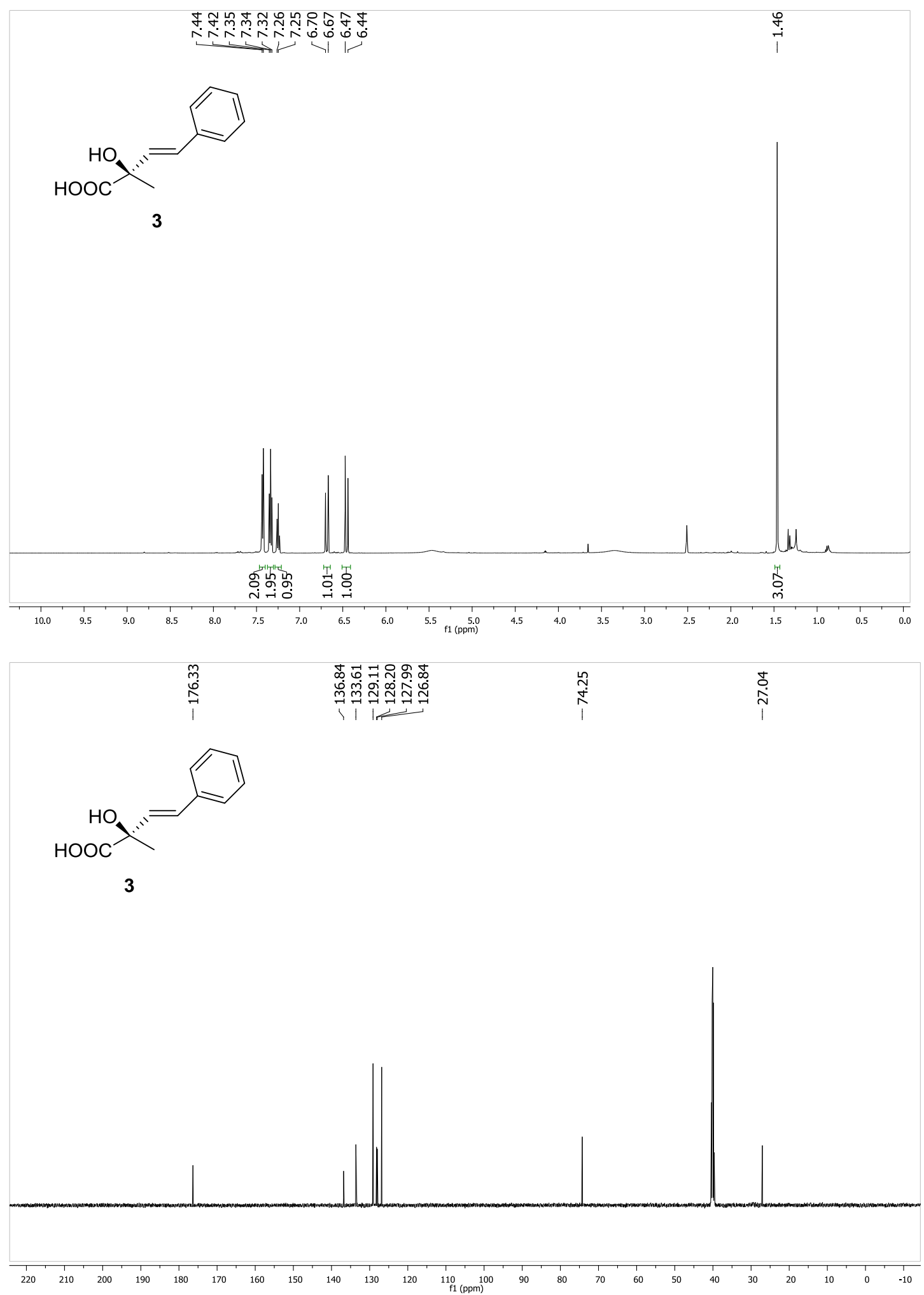

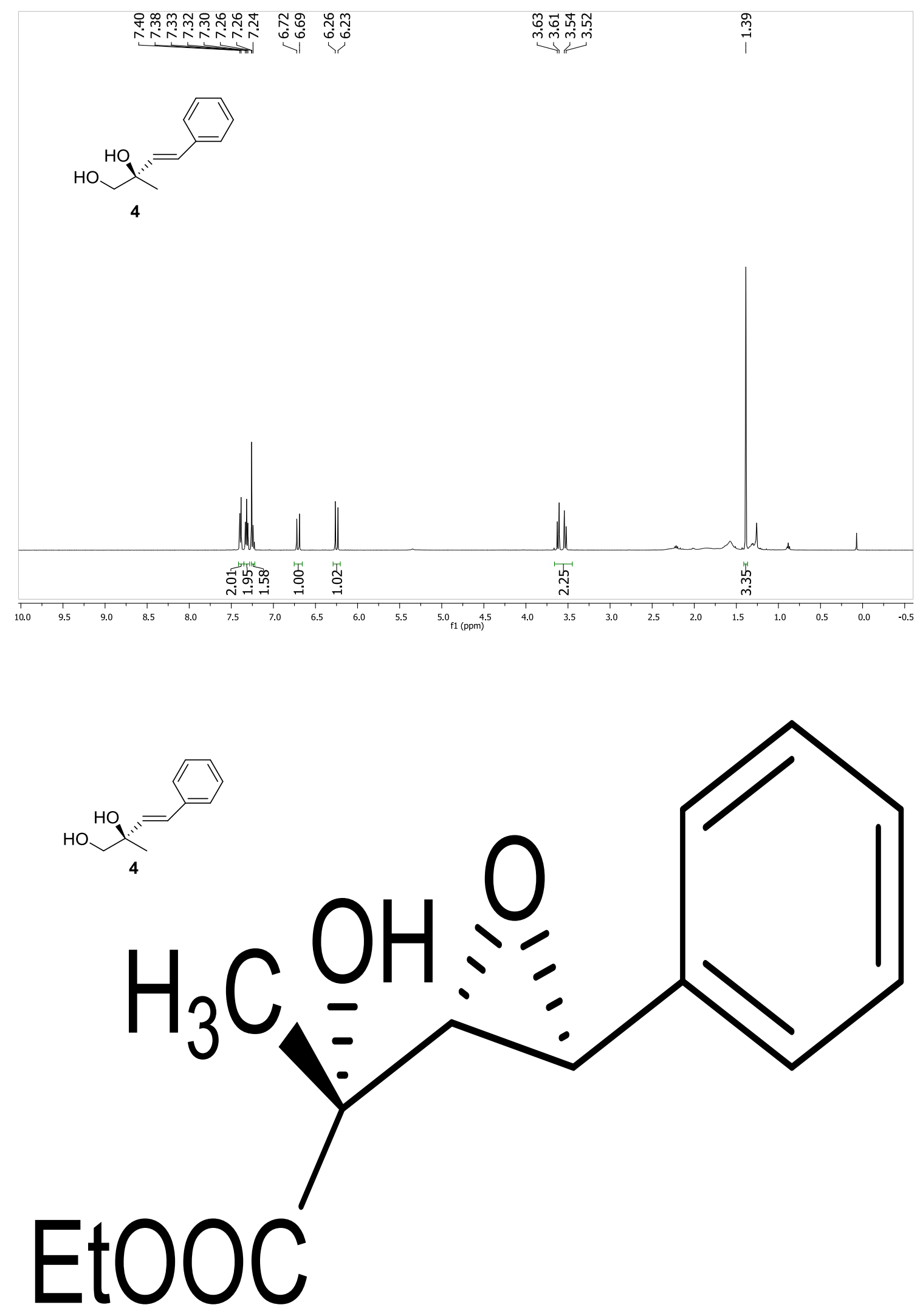

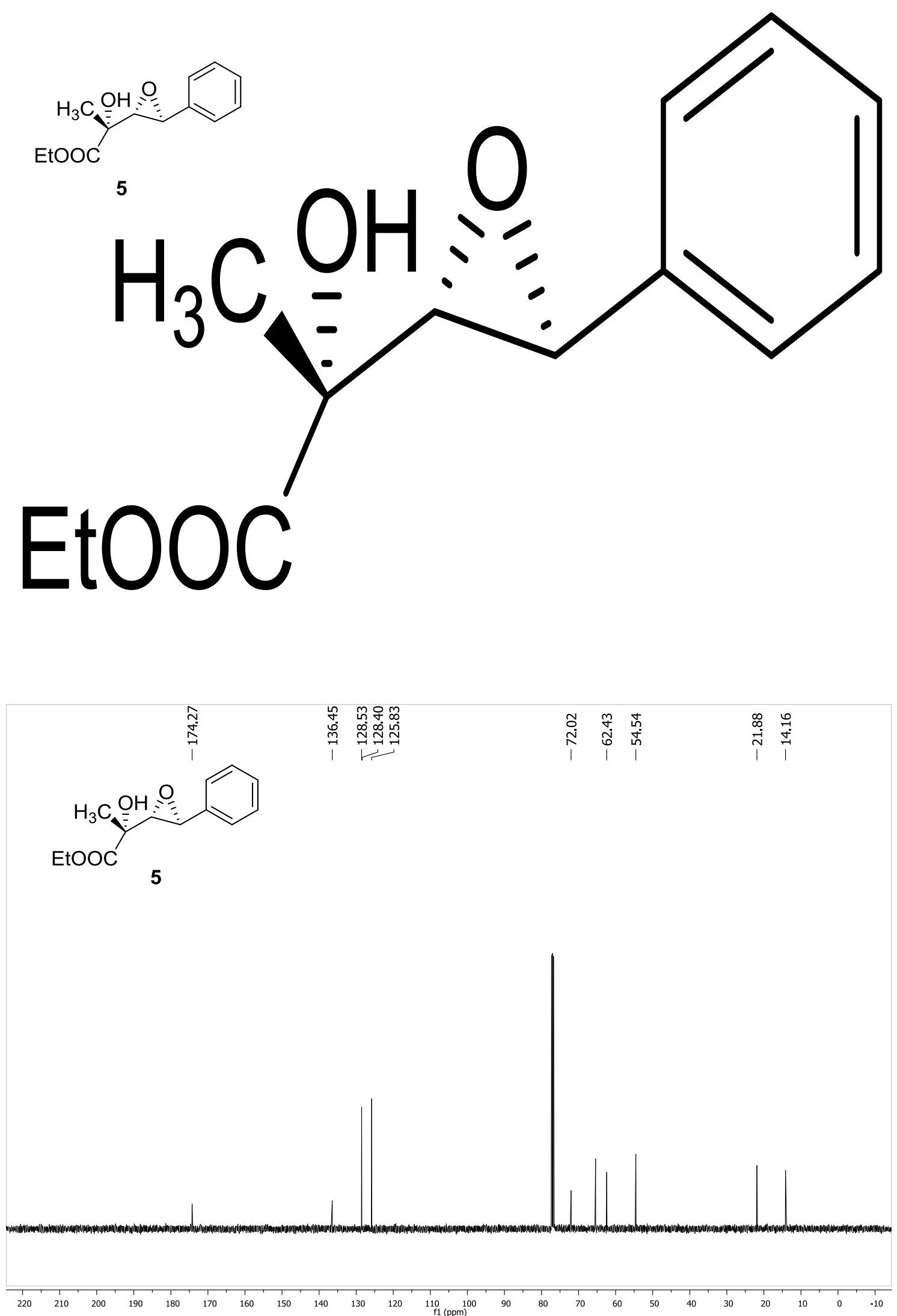
2-D NMR:

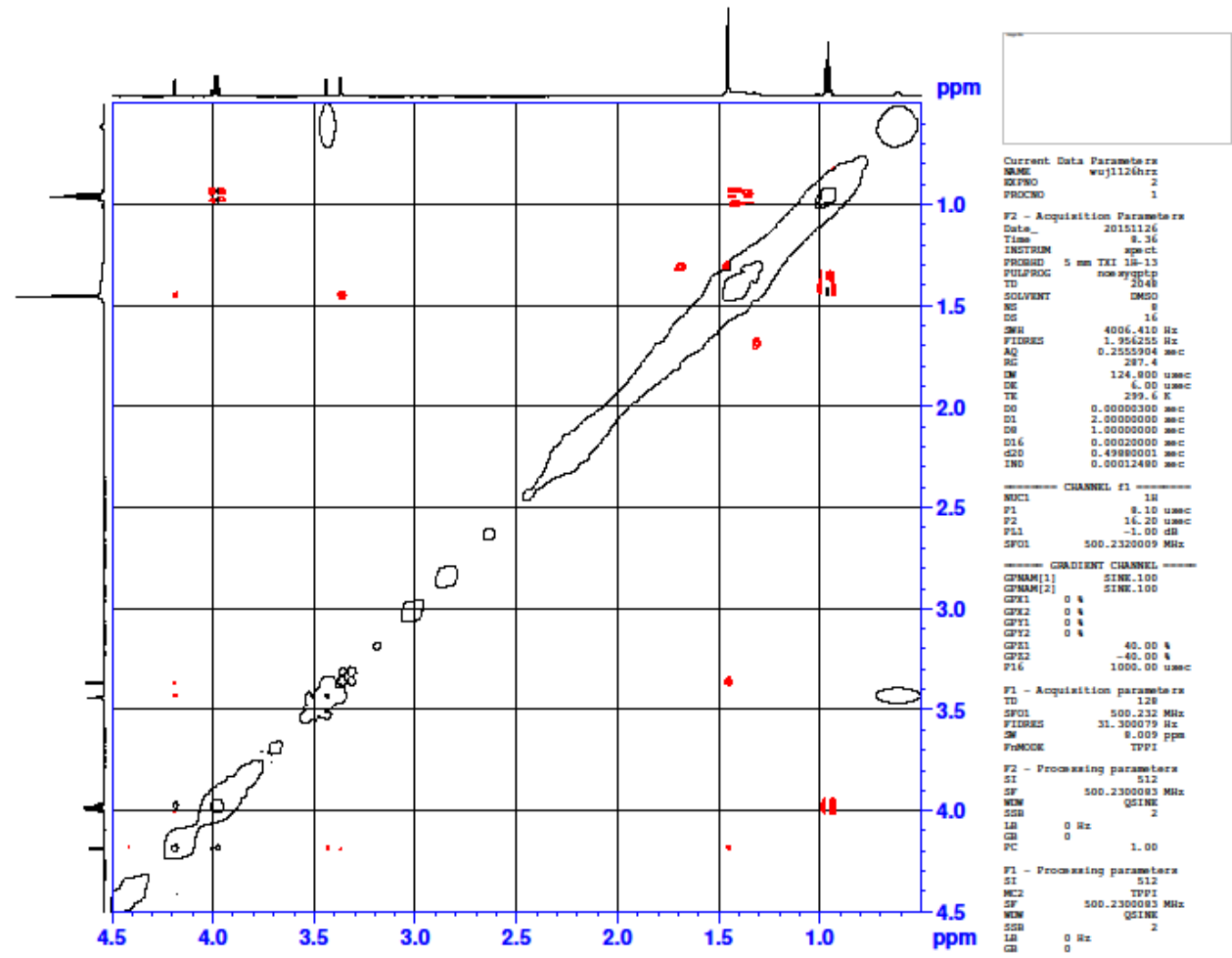



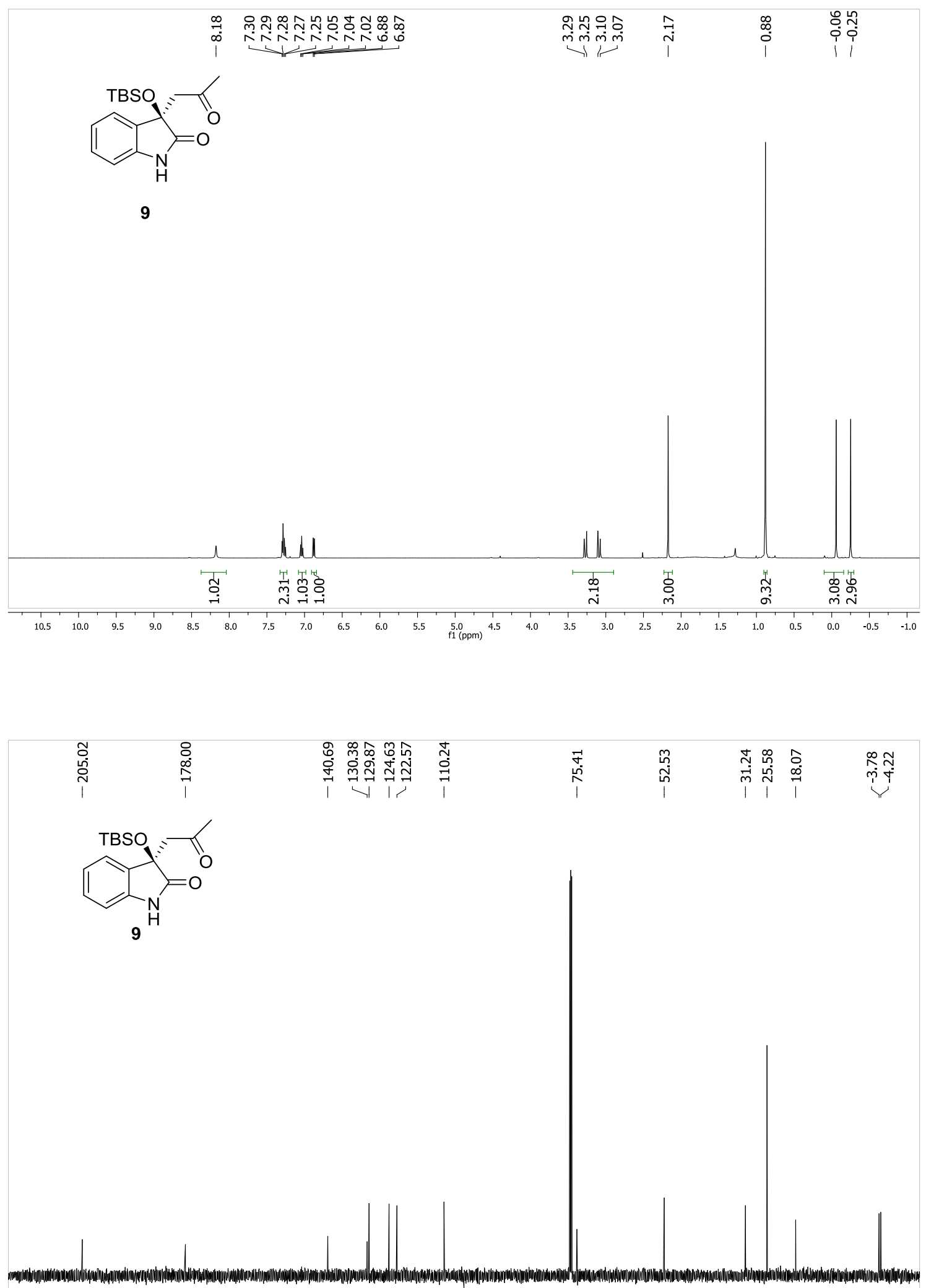


\section{X-ray analysis of compound 3}
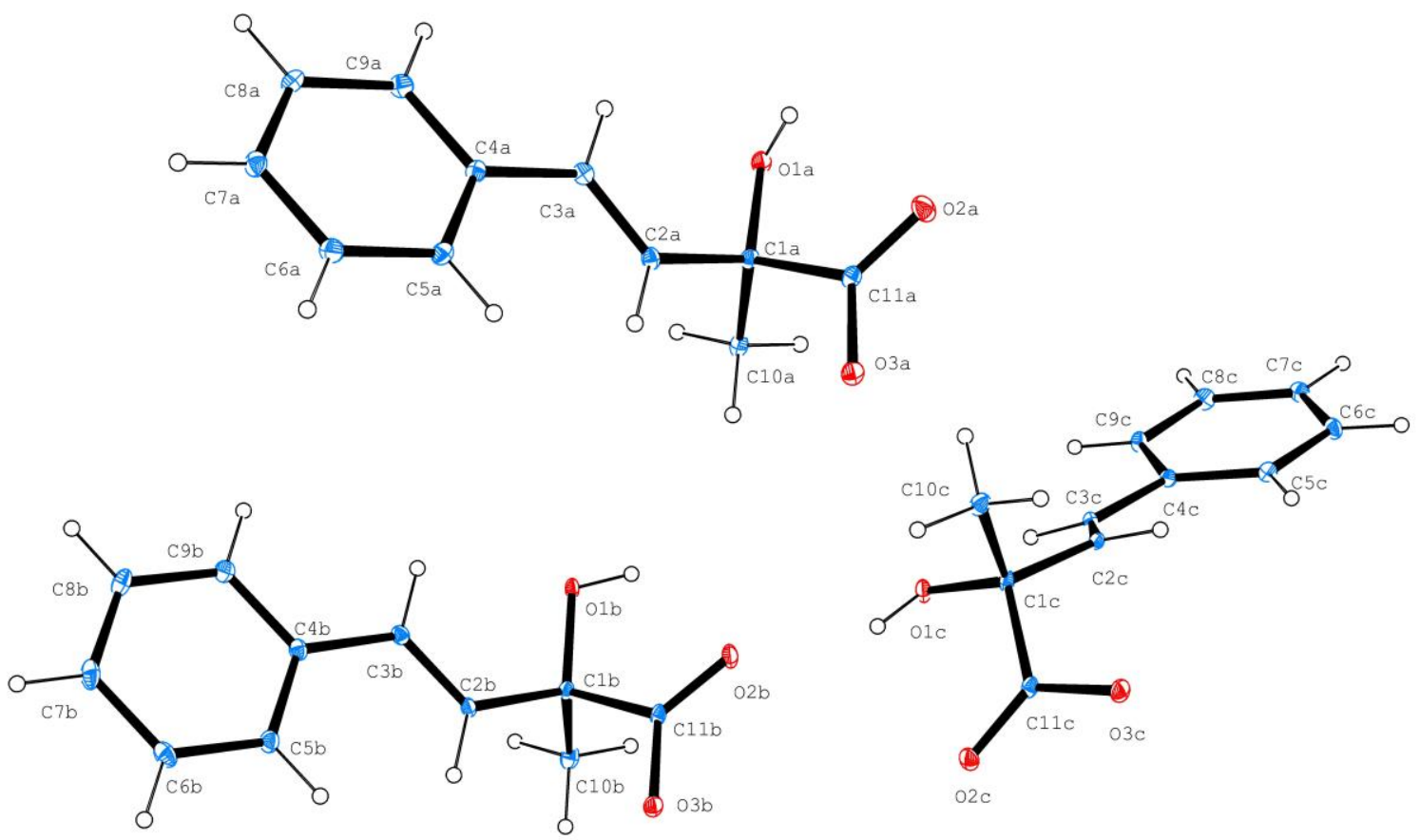

Molecule's view

Table S1. Crystal data and structure refinement for $\mathrm{f} 498$.

Empirical formula

Formula weight

Temperature

Wavelength

Crystal system

Space group

Unit cell dimensions

Volume

Z

Density (calculated)

Absorption coefficient
$\mathrm{C}_{33} \mathrm{H}_{36} \mathrm{O}_{9}$

576.62

100(2) K

$1.54178 \AA$

Orthorhombic

P 2(1) 2(1) 2(1)

$\mathrm{a}=5.8398(2) \AA$

$\alpha=90^{\circ}$

$\mathrm{b}=21.5974(8) \AA$

$\beta=90^{\circ}$

$\mathrm{c}=23.1903(8) \AA$

$\gamma=90^{\circ}$

2924.87(18) $\AA^{3}$

4

$1.309 \mathrm{Mg} / \mathrm{m}^{3}$

$0.783 \mathrm{~mm}^{-1}$ 
$\mathrm{F}(000)$

Crystal size

Theta range for data collection

Index ranges

Reflections collected

Independent reflections

Completeness to theta $=67.679^{\circ}$

Refinement method

Data / restraints / parameters

Goodness-of-fit on $\mathrm{F}^{2}$

Final R indices [I $>2 \operatorname{sigma}(\mathrm{I})]$

$\mathrm{R}$ indices (all data)

Absolute structure parameter

Largest diff. peak and hole
1224

$0.361 \times 0.133 \times 0.090 \mathrm{~mm}^{3}$

2.796 to $72.458^{\circ}$

$-7<=\mathrm{h}<=6,-18<=\mathrm{k}<=26,-28<=\mathrm{l}<=24$

14205

$5676[\mathrm{R}(\mathrm{int})=0.0354]$

$99.6 \%$

Full-matrix least-squares on $\mathrm{F}^{2}$

$5676 / 0 / 385$

1.054

$\mathrm{R} 1=0.0377, \mathrm{wR} 2=0.0929$

$\mathrm{R} 1=0.0428, \mathrm{wR} 2=0.0962$

$0.00(8)^{*}$

0.260 and -0.224 e. $\AA^{-3}$

*Flack x determined using 2042 quotients [(I+)-(I-)]/[(I+)+(I-)]

(Parsons, Flack and Wagner, Acta Cryst. B69 (2013) 249-259). 
Table S2. Atomic coordinates $\left(x 1^{4}\right)$ and equivalent isotropic displacement parameters $\left(\AA^{2} x 10^{3}\right)$ for $4498 . \quad U(e q)$ is defined as one third of the trace of the orthogonalized $U^{i j}$ tensor.

\begin{tabular}{|c|c|c|c|c|}
\hline & $\mathrm{x}$ & $\mathrm{y}$ & $\mathrm{z}$ & $\mathrm{U}(\mathrm{eq})$ \\
\hline $\mathrm{O}(1 \mathrm{~A})$ & $7408(3)$ & $3312(1)$ & $5180(1)$ & $17(1)$ \\
\hline $\mathrm{O}(2 \mathrm{~A})$ & $3192(3)$ & $2927(1)$ & $4932(1)$ & $27(1)$ \\
\hline $\mathrm{O}(3 \mathrm{~A})$ & $1561(3)$ & $3681(1)$ & $5453(1)$ & $20(1)$ \\
\hline $\mathrm{C}(1 \mathrm{~A})$ & $5600(4)$ & $3632(1)$ & $5465(1)$ & $15(1)$ \\
\hline$C(2 A)$ & $5694(4)$ & $3562(1)$ & $6115(1)$ & $15(1)$ \\
\hline $\mathrm{C}(3 \mathrm{~A})$ & 7249(4) & $3228(1)$ & $6393(1)$ & $16(1)$ \\
\hline $\mathrm{C}(4 \mathrm{~A})$ & $7547(4)$ & $3192(1)$ & $7024(1)$ & $16(1)$ \\
\hline $\mathrm{C}(5 \mathrm{~A})$ & $5896(5)$ & $3396(1)$ & $7414(1)$ & $17(1)$ \\
\hline$C(6 A)$ & $6323(5)$ & $3378(1)$ & $8006(1)$ & $20(1)$ \\
\hline $\mathrm{C}(7 \mathrm{~A})$ & $8404(5)$ & $3160(1)$ & $8212(1)$ & $20(1)$ \\
\hline $\mathrm{C}(8 \mathrm{~A})$ & $10041(5)$ & $2946(1)$ & $7828(1)$ & $20(1)$ \\
\hline $\mathrm{C}(9 \mathrm{~A})$ & $9610(5)$ & $2960(1)$ & $7239(1)$ & 19(1) \\
\hline$C(10 A)$ & $5840(4)$ & $4314(1)$ & $5297(1)$ & $18(1)$ \\
\hline \multirow[t]{2}{*}{$\mathrm{C}(11 \mathrm{~A})$} & $3315(4)$ & $3371(1)$ & $5250(1)$ & $16(1)$ \\
\hline & $\mathrm{x}$ & $\mathrm{y}$ & $\mathrm{z}$ & $\mathrm{U}(\mathrm{eq})$ \\
\hline $\mathrm{O}(1 \mathrm{~B})$ & 7498(3) & $6433(1)$ & $5605(1)$ & $17(1)$ \\
\hline $\mathrm{O}(2 \mathrm{~B})$ & $3299(3)$ & $6158(1)$ & $5240(1)$ & $21(1)$ \\
\hline $\mathrm{O}(3 \mathrm{~B})$ & 1659(3) & $6836(1)$ & $5845(1)$ & 19(1) \\
\hline $\mathrm{C}(1 \mathrm{~B})$ & 5706(4) & $6820(1)$ & $5818(1)$ & $14(1)$ \\
\hline $\mathrm{C}(2 \mathrm{~B})$ & $5769(4)$ & $6819(1)$ & $6470(1)$ & $14(1)$ \\
\hline $\mathrm{C}(3 \mathrm{~B})$ & $7242(4)$ & $6499(1)$ & $6787(1)$ & $15(1)$ \\
\hline $\mathrm{C}(4 \mathrm{~B})$ & 7404(5) & $6509(1)$ & $7422(1)$ & $16(1)$ \\
\hline $\mathrm{C}(5 \mathrm{~B})$ & $5696(5)$ & $6759(1)$ & $7775(1)$ & $18(1)$ \\
\hline $\mathrm{C}(6 \mathrm{~B})$ & $6001(5)$ & $6783(1)$ & $8371(1)$ & $23(1)$ \\
\hline $\mathrm{C}(7 \mathrm{~B})$ & 7992(5) & $6557(1)$ & $8619(1)$ & $25(1)$ \\
\hline $\mathrm{C}(8 \mathrm{~B})$ & $9661(5)$ & $6296(1)$ & $8277(1)$ & $25(1)$ \\
\hline $\mathrm{C}(9 \mathrm{~B})$ & $9377(5)$ & $6272(1)$ & $7679(1)$ & $20(1)$ \\
\hline & $\mathrm{x}$ & $\mathrm{y}$ & $\mathrm{z}$ & $\mathrm{U}(\mathrm{eq})$ \\
\hline
\end{tabular}




\begin{tabular}{|c|c|c|c|c|}
\hline$C(10 B)$ & 5973(5) & 7481(1) & $5585(1)$ & $21(1)$ \\
\hline \multirow[t]{2}{*}{$C(11 B)$} & $3419(4)$ & $6563(1)$ & $5605(1)$ & $14(1)$ \\
\hline & $\mathrm{x}$ & $\mathrm{y}$ & $\mathrm{z}$ & $\mathrm{U}(\mathrm{eq})$ \\
\hline $\mathrm{O}(1 \mathrm{C})$ & 2411(3) & $5687(1)$ & $4157(1)$ & $18(1)$ \\
\hline $\mathrm{O}(2 \mathrm{C})$ & $-1790(3)$ & $6027(1)$ & $4472(1)$ & $20(1)$ \\
\hline $\mathrm{O}(3 \mathrm{C})$ & $-3422(3)$ & $5248(1)$ & $3979(1)$ & $20(1)$ \\
\hline $\mathrm{C}(1 \mathrm{C})$ & $627(4)$ & $5264(1)$ & $4018(1)$ & $15(1)$ \\
\hline $\mathrm{C}(2 \mathrm{C})$ & $760(4)$ & $5109(1)$ & $3384(1)$ & $14(1)$ \\
\hline $\mathrm{C}(3 \mathrm{C})$ & 2334(4) & $5342(1)$ & $3034(1)$ & $15(1)$ \\
\hline $\mathrm{C}(4 \mathrm{C})$ & 2693(4) & $5185(1)$ & $2420(1)$ & $14(1)$ \\
\hline $\mathrm{C}(5 \mathrm{C})$ & 1068(4) & $4874(1)$ & 2085(1) & $16(1)$ \\
\hline $\mathrm{C}(6 \mathrm{C})$ & 1552(5) & $4724(1)$ & $1515(1)$ & $19(1)$ \\
\hline $\mathrm{C}(7 \mathrm{C})$ & $3647(5)$ & $4876(1)$ & 1274(1) & $19(1)$ \\
\hline $\mathrm{C}(8 \mathrm{C})$ & $5262(5)$ & $5188(1)$ & 1601(1) & $20(1)$ \\
\hline $\mathrm{C}(9 \mathrm{C})$ & 4777(5) & $5347(1)$ & 2170(1) & $17(1)$ \\
\hline$C(10 C)$ & $864(5)$ & $4668(1)$ & $4375(1)$ & $20(1)$ \\
\hline$C(11 C)$ & $-1664(4)$ & $5562(1)$ & $4177(1)$ & $15(1)$ \\
\hline
\end{tabular}

Table S4. Bond lengths [A] and angles $\left[^{\circ}\right]$ for 4498.

$\begin{array}{llll}\mathrm{O}(1 \mathrm{~A})-\mathrm{C}(1 \mathrm{~A}) & 1.424(3) & \mathrm{O}(1 \mathrm{~A})-\mathrm{H}(1 \mathrm{~A}) & 0.8400 \\ \mathrm{O}(2 \mathrm{~A})-\mathrm{C}(11 \mathrm{~A}) & 1.210(3) & \mathrm{O}(3 \mathrm{~A})-\mathrm{C}(11 \mathrm{~A}) & 1.312(3) \\ \mathrm{O}(3 \mathrm{~A})-\mathrm{H}(3 \mathrm{~A}) & 0.8400 & \mathrm{C}(1 \mathrm{~A})-\mathrm{C}(2 \mathrm{~A}) & 1.516(3) \\ \mathrm{C}(1 \mathrm{~A})-\mathrm{C}(10 \mathrm{~A}) & 1.529(3) & \mathrm{C}(1 \mathrm{~A})-\mathrm{C}(11 \mathrm{~A}) & 1.532(3) \\ \mathrm{C}(2 \mathrm{~A})-\mathrm{C}(3 \mathrm{~A}) & 1.327(3) & \mathrm{C}(2 \mathrm{~A})-\mathrm{H}(2 \mathrm{~A}) & 0.9500 \\ \mathrm{C}(3 \mathrm{~A})-\mathrm{C}(4 \mathrm{~A}) & 1.476(3) & \mathrm{C}(3 \mathrm{~A})-\mathrm{H}(3 \mathrm{~A} 1) & 0.9500 \\ \mathrm{C}(4 \mathrm{~A})-\mathrm{C}(5 \mathrm{~A}) & 1.394(3) & \mathrm{C}(4 \mathrm{~A})-\mathrm{C}(9 \mathrm{~A}) & 1.396(4) \\ \mathrm{C}(5 \mathrm{~A})-\mathrm{C}(6 \mathrm{~A}) & 1.395(3) & \mathrm{C}(5 \mathrm{~A})-\mathrm{H}(5 \mathrm{~A}) & 0.9500 \\ \mathrm{C}(6 \mathrm{~A})-\mathrm{C}(7 \mathrm{~A}) & 1.388(4) & \mathrm{C}(6 \mathrm{~A})-\mathrm{H}(6 \mathrm{~A}) & 0.9500 \\ \mathrm{C}(7 \mathrm{~A})-\mathrm{C}(8 \mathrm{~A}) & 1.386(4) & \mathrm{C}(7 \mathrm{~A})-\mathrm{H}(7 \mathrm{~A}) & 0.9500 \\ \mathrm{C}(8 \mathrm{~A})-\mathrm{C}(9 \mathrm{~A}) & 1.391(3) & \mathrm{C}(8 \mathrm{~A})-\mathrm{H}(8 \mathrm{~A}) & 0.9500\end{array}$




\begin{tabular}{|c|c|c|c|}
\hline $\mathrm{C}(9 \mathrm{~A})-\mathrm{H}(9 \mathrm{~A})$ & 0.9500 & $\mathrm{C}(10 \mathrm{~A})-\mathrm{H}(10 \mathrm{~A})$ & 0.9800 \\
\hline $\mathrm{C}(10 \mathrm{~A})-\mathrm{H}(10 \mathrm{~B})$ & 0.9800 & $\mathrm{C}(10 \mathrm{~A})-\mathrm{H}(10 \mathrm{C})$ & 0.9800 \\
\hline $\mathrm{O}(1 \mathrm{~B})-\mathrm{C}(1 \mathrm{~B})$ & $1.427(3)$ & $\mathrm{O}(1 \mathrm{~B})-\mathrm{H}(1 \mathrm{~B})$ & 0.8400 \\
\hline $\mathrm{O}(2 \mathrm{~B})-\mathrm{C}(11 \mathrm{~B})$ & $1.220(3)$ & $\mathrm{O}(3 \mathrm{~B})-\mathrm{C}(11 \mathrm{~B})$ & $1.310(3)$ \\
\hline $\mathrm{O}(3 \mathrm{~B})-\mathrm{H}(3 \mathrm{~B})$ & 0.8400 & $\mathrm{C}(1 \mathrm{~B})-\mathrm{C}(2 \mathrm{~B})$ & $1.513(3)$ \\
\hline$C(1 B)-C(11 B)$ & $1.528(3)$ & $C(1 B)-C(10 B)$ & $1.535(3)$ \\
\hline$C(2 B)-C(3 B)$ & $1.325(3)$ & $\mathrm{C}(2 \mathrm{~B})-\mathrm{H}(2 \mathrm{~B})$ & 0.9500 \\
\hline$C(3 B)-C(4 B)$ & $1.477(3)$ & $\mathrm{C}(3 \mathrm{~B})-\mathrm{H}(3 \mathrm{~B} 1)$ & 0.9500 \\
\hline $\mathrm{C}(4 \mathrm{~B})-\mathrm{C}(9 \mathrm{~B})$ & $1.395(4)$ & $\mathrm{C}(4 \mathrm{~B})-\mathrm{C}(5 \mathrm{~B})$ & $1.398(3)$ \\
\hline$C(5 B)-C(6 B)$ & $1.394(3)$ & $\mathrm{C}(5 \mathrm{~B})-\mathrm{H}(5 \mathrm{~B})$ & 0.9500 \\
\hline$C(6 B)-C(7 B)$ & $1.386(4)$ & $\mathrm{C}(6 \mathrm{~B})-\mathrm{H}(6 \mathrm{~B})$ & 0.9500 \\
\hline$C(7 B)-C(8 B)$ & $1.378(4)$ & $\mathrm{C}(7 \mathrm{~B})-\mathrm{H}(7 \mathrm{~B})$ & 0.9500 \\
\hline$C(8 B)-C(9 B)$ & $1.395(3)$ & $\mathrm{C}(8 \mathrm{~B})-\mathrm{H}(8 \mathrm{~B})$ & 0.9500 \\
\hline $\mathrm{C}(9 \mathrm{~B})-\mathrm{H}(9 \mathrm{~B})$ & 0.9500 & $\mathrm{C}(10 \mathrm{~B})-\mathrm{H}(10 \mathrm{D})$ & 0.9800 \\
\hline $\mathrm{C}(10 \mathrm{~B})-\mathrm{H}(10 \mathrm{E})$ & 0.9800 & $\mathrm{C}(10 \mathrm{~B})-\mathrm{H}(10 \mathrm{~F})$ & 0.9800 \\
\hline $\mathrm{O}(1 \mathrm{C})-\mathrm{C}(1 \mathrm{C})$ & $1.422(3)$ & $\mathrm{O}(1 \mathrm{C})-\mathrm{H}(1 \mathrm{C})$ & 0.8400 \\
\hline $\mathrm{O}(2 \mathrm{C})-\mathrm{C}(11 \mathrm{C})$ & $1.219(3)$ & $\mathrm{O}(3 \mathrm{C})-\mathrm{C}(11 \mathrm{C})$ & $1.314(3)$ \\
\hline $\mathrm{O}(3 \mathrm{C})-\mathrm{H}(3 \mathrm{C})$ & 0.8400 & $C(1 C)-C(2 C)$ & $1.512(3)$ \\
\hline$C(1 C)-C(11 C)$ & $1.528(3)$ & $C(1 C)-C(10 C)$ & $1.537(3)$ \\
\hline$C(2 C)-C(3 C)$ & $1.325(3)$ & $\mathrm{C}(2 \mathrm{C})-\mathrm{H}(2 \mathrm{C})$ & 0.9500 \\
\hline$C(3 C)-C(4 C)$ & $1.480(3)$ & $\mathrm{C}(3 \mathrm{C})-\mathrm{H}(3 \mathrm{C} 1)$ & 0.9500 \\
\hline$C(4 C)-C(9 C)$ & $1.393(3)$ & $C(4 C)-C(5 C)$ & $1.398(3)$ \\
\hline$C(5 C)-C(6 C)$ & $1.391(3)$ & $\mathrm{C}(5 \mathrm{C})-\mathrm{H}(5 \mathrm{C})$ & 0.9500 \\
\hline$C(6 C)-C(7 C)$ & $1.384(4)$ & $\mathrm{C}(6 \mathrm{C})-\mathrm{H}(6 \mathrm{C})$ & 0.9500 \\
\hline$C(7 C)-C(8 C)$ & $1.386(4)$ & $\mathrm{C}(7 \mathrm{C})-\mathrm{H}(7 \mathrm{C})$ & 0.9500 \\
\hline$C(8 C)-C(9 C)$ & $1.393(3)$ & $\mathrm{C}(8 \mathrm{C})-\mathrm{H}(8 \mathrm{C})$ & 0.9500 \\
\hline $\mathrm{C}(9 \mathrm{C})-\mathrm{H}(9 \mathrm{C})$ & 0.9500 & $\mathrm{C}(10 \mathrm{C})-\mathrm{H}(10 \mathrm{G})$ & 0.9800 \\
\hline $\mathrm{C}(10 \mathrm{C})-\mathrm{H}(10 \mathrm{H})$ & 0.9800 & $\mathrm{C}(10 \mathrm{C})-\mathrm{H}(10 \mathrm{I})$ & 0.9800 \\
\hline $\mathrm{C}(1 \mathrm{~A})-\mathrm{O}(1 \mathrm{~A})-\mathrm{H}(1 \mathrm{~A})$ & 109.5 & $\mathrm{C}(11 \mathrm{~A})-\mathrm{O}(3 \mathrm{~A})-\mathrm{H}(3 \mathrm{~A})$ & 109.5 \\
\hline $\mathrm{O}(1 \mathrm{~A})-\mathrm{C}(1 \mathrm{~A})-\mathrm{C}(2 \mathrm{~A})$ & $112.7(2)$ & $O(1 \mathrm{~A})-\mathrm{C}(1 \mathrm{~A})-\mathrm{C}(10 \mathrm{~A})$ & $106.35(19)$ \\
\hline$C(2 A)-C(1 A)-C(10 A)$ & $110.28(18)$ & $\mathrm{O}(1 \mathrm{~A})-\mathrm{C}(1 \mathrm{~A})-\mathrm{C}(11 \mathrm{~A})$ & $108.42(17)$ \\
\hline$C(2 A)-C(1 A)-C(11 A)$ & 108.52(19) & $C(10 A)-C(1 A)-C(11 A)$ & $110.6(2)$ \\
\hline$C(3 \mathrm{~A})-\mathrm{C}(2 \mathrm{~A})-\mathrm{C}(1 \mathrm{~A})$ & $124.3(2)$ & $\mathrm{C}(3 \mathrm{~A})-\mathrm{C}(2 \mathrm{~A})-\mathrm{H}(2 \mathrm{~A})$ & 117.9 \\
\hline $\mathrm{C}(1 \mathrm{~A})-\mathrm{C}(2 \mathrm{~A})-\mathrm{H}(2 \mathrm{~A})$ & 117.9 & $\mathrm{C}(2 \mathrm{~A})-\mathrm{C}(3 \mathrm{~A})-\mathrm{C}(4 \mathrm{~A})$ & $126.3(2)$ \\
\hline $\mathrm{C}(2 \mathrm{~A})-\mathrm{C}(3 \mathrm{~A})-\mathrm{H}(3 \mathrm{~A} 1)$ & 116.8 & $\mathrm{C}(4 \mathrm{~A})-\mathrm{C}(3 \mathrm{~A})-\mathrm{H}(3 \mathrm{~A} 1)$ & 116.8 \\
\hline
\end{tabular}




\begin{tabular}{|c|c|c|c|}
\hline$C(5 A)-C(4 A)-C(9 A)$ & $118.6(2)$ & $\mathrm{C}(5 \mathrm{~A})-\mathrm{C}(4 \mathrm{~A})-\mathrm{C}(3 \mathrm{~A})$ & $123.1(2)$ \\
\hline$C(9 A)-C(4 A)-C(3 A)$ & $118.3(2)$ & $C(4 A)-C(5 A)-C(6 A)$ & $120.4(2)$ \\
\hline $\mathrm{C}(4 \mathrm{~A})-\mathrm{C}(5 \mathrm{~A})-\mathrm{H}(5 \mathrm{~A})$ & 119.8 & $\mathrm{C}(6 \mathrm{~A})-\mathrm{C}(5 \mathrm{~A})-\mathrm{H}(5 \mathrm{~A})$ & 119.8 \\
\hline$C(7 A)-C(6 A)-C(5 A)$ & $120.3(2)$ & $C(7 A)-C(6 A)-H(6 A)$ & 119.8 \\
\hline $\mathrm{C}(5 \mathrm{~A})-\mathrm{C}(6 \mathrm{~A})-\mathrm{H}(6 \mathrm{~A})$ & 119.8 & $\mathrm{C}(8 \mathrm{~A})-\mathrm{C}(7 \mathrm{~A})-\mathrm{C}(6 \mathrm{~A})$ & $119.7(2)$ \\
\hline $\mathrm{C}(8 \mathrm{~A})-\mathrm{C}(7 \mathrm{~A})-\mathrm{H}(7 \mathrm{~A})$ & 120.1 & $\mathrm{C}(6 \mathrm{~A})-\mathrm{C}(7 \mathrm{~A})-\mathrm{H}(7 \mathrm{~A})$ & 120.1 \\
\hline$C(7 A)-C(8 A)-C(9 A)$ & $120.0(2)$ & $\mathrm{C}(7 \mathrm{~A})-\mathrm{C}(8 \mathrm{~A})-\mathrm{H}(8 \mathrm{~A})$ & 120.0 \\
\hline $\mathrm{C}(9 \mathrm{~A})-\mathrm{C}(8 \mathrm{~A})-\mathrm{H}(8 \mathrm{~A})$ & 120.0 & $C(8 A)-C(9 A)-C(4 A)$ & $120.9(2)$ \\
\hline $\mathrm{C}(8 \mathrm{~A})-\mathrm{C}(9 \mathrm{~A})-\mathrm{H}(9 \mathrm{~A})$ & 119.5 & $\mathrm{C}(4 \mathrm{~A})-\mathrm{C}(9 \mathrm{~A})-\mathrm{H}(9 \mathrm{~A})$ & 119.5 \\
\hline$C(1 \mathrm{~A})-\mathrm{C}(10 \mathrm{~A})-\mathrm{H}(10 \mathrm{~A})$ & 109.5 & $C(1 A)-C(10 A)-H(10 B)$ & 109.5 \\
\hline $\mathrm{H}(10 \mathrm{~A})-\mathrm{C}(10 \mathrm{~A})-\mathrm{H}(10 \mathrm{~B})$ & 109.5 & $\mathrm{C}(1 \mathrm{~A})-\mathrm{C}(10 \mathrm{~A})-\mathrm{H}(10 \mathrm{C})$ & 109.5 \\
\hline $\mathrm{H}(10 \mathrm{~A})-\mathrm{C}(10 \mathrm{~A})-\mathrm{H}(10 \mathrm{C})$ & 109.5 & $\mathrm{H}(10 \mathrm{~B})-\mathrm{C}(10 \mathrm{~A})-\mathrm{H}(10 \mathrm{C})$ & 109.5 \\
\hline $\mathrm{O}(2 \mathrm{~A})-\mathrm{C}(11 \mathrm{~A})-\mathrm{O}(3 \mathrm{~A})$ & $125.2(2)$ & $\mathrm{O}(2 \mathrm{~A})-\mathrm{C}(11 \mathrm{~A})-\mathrm{C}(1 \mathrm{~A})$ & $122.8(2)$ \\
\hline $\mathrm{O}(3 \mathrm{~A})-\mathrm{C}(11 \mathrm{~A})-\mathrm{C}(1 \mathrm{~A})$ & $112.00(18)$ & & \\
\hline $\mathrm{C}(1 \mathrm{~B})-\mathrm{O}(1 \mathrm{~B})-\mathrm{H}(1 \mathrm{~B})$ & 109.5 & $\mathrm{C}(11 \mathrm{~B})-\mathrm{O}(3 \mathrm{~B})-\mathrm{H}(3 \mathrm{~B})$ & 109.5 \\
\hline $\mathrm{O}(1 \mathrm{~B})-\mathrm{C}(1 \mathrm{~B})-\mathrm{C}(2 \mathrm{~B})$ & $109.11(19)$ & $\mathrm{O}(1 \mathrm{~B})-\mathrm{C}(1 \mathrm{~B})-\mathrm{C}(11 \mathrm{~B})$ & $108.42(18)$ \\
\hline$C(2 B)-C(1 B)-C(11 B)$ & $110.15(19)$ & $\mathrm{O}(1 \mathrm{~B})-\mathrm{C}(1 \mathrm{~B})-\mathrm{C}(10 \mathrm{~B})$ & $110.40(19)$ \\
\hline$C(2 B)-C(1 B)-C(10 B)$ & $110.56(19)$ & $C(11 B)-C(1 B)-C(10 B)$ & $108.2(2)$ \\
\hline $\mathrm{C}(3 \mathrm{~B})-\mathrm{C}(2 \mathrm{~B})-\mathrm{C}(1 \mathrm{~B})$ & $124.8(2)$ & $\mathrm{C}(3 \mathrm{~B})-\mathrm{C}(2 \mathrm{~B})-\mathrm{H}(2 \mathrm{~B})$ & 117.6 \\
\hline $\mathrm{C}(1 \mathrm{~B})-\mathrm{C}(2 \mathrm{~B})-\mathrm{H}(2 \mathrm{~B})$ & 117.6 & $\mathrm{C}(2 \mathrm{~B})-\mathrm{C}(3 \mathrm{~B})-\mathrm{C}(4 \mathrm{~B})$ & $125.9(2)$ \\
\hline $\mathrm{C}(2 \mathrm{~B})-\mathrm{C}(3 \mathrm{~B})-\mathrm{H}(3 \mathrm{~B} 1)$ & 117.0 & $\mathrm{C}(4 \mathrm{~B})-\mathrm{C}(3 \mathrm{~B})-\mathrm{H}(3 \mathrm{~B} 1)$ & 117.0 \\
\hline $\mathrm{C}(9 \mathrm{~B})-\mathrm{C}(4 \mathrm{~B})-\mathrm{C}(5 \mathrm{~B})$ & $118.8(2)$ & $\mathrm{C}(9 \mathrm{~B})-\mathrm{C}(4 \mathrm{~B})-\mathrm{C}(3 \mathrm{~B})$ & $118.3(2)$ \\
\hline$C(5 B)-C(4 B)-C(3 B)$ & $122.9(2)$ & $C(6 B)-C(5 B)-C(4 B)$ & $120.1(2)$ \\
\hline $\mathrm{C}(6 \mathrm{~B})-\mathrm{C}(5 \mathrm{~B})-\mathrm{H}(5 \mathrm{~B})$ & 119.9 & $\mathrm{C}(4 \mathrm{~B})-\mathrm{C}(5 \mathrm{~B})-\mathrm{H}(5 \mathrm{~B})$ & 119.9 \\
\hline$C(7 B)-C(6 B)-C(5 B)$ & $120.5(2)$ & $\mathrm{C}(7 \mathrm{~B})-\mathrm{C}(6 \mathrm{~B})-\mathrm{H}(6 \mathrm{~B})$ & 119.8 \\
\hline$C(5 B)-C(6 B)-H(6 B)$ & 119.8 & $\mathrm{C}(8 \mathrm{~B})-\mathrm{C}(7 \mathrm{~B})-\mathrm{C}(6 \mathrm{~B})$ & $119.8(2)$ \\
\hline $\mathrm{C}(8 \mathrm{~B})-\mathrm{C}(7 \mathrm{~B})-\mathrm{H}(7 \mathrm{~B})$ & 120.1 & $\mathrm{C}(6 \mathrm{~B})-\mathrm{C}(7 \mathrm{~B})-\mathrm{H}(7 \mathrm{~B})$ & 120.1 \\
\hline $\mathrm{C}(7 \mathrm{~B})-\mathrm{C}(8 \mathrm{~B})-\mathrm{C}(9 \mathrm{~B})$ & $120.2(3)$ & $\mathrm{C}(7 \mathrm{~B})-\mathrm{C}(8 \mathrm{~B})-\mathrm{H}(8 \mathrm{~B})$ & 119.9 \\
\hline $\mathrm{C}(9 \mathrm{~B})-\mathrm{C}(8 \mathrm{~B})-\mathrm{H}(8 \mathrm{~B})$ & 119.9 & $\mathrm{C}(4 \mathrm{~B})-\mathrm{C}(9 \mathrm{~B})-\mathrm{C}(8 \mathrm{~B})$ & $120.5(3)$ \\
\hline $\mathrm{C}(4 \mathrm{~B})-\mathrm{C}(9 \mathrm{~B})-\mathrm{H}(9 \mathrm{~B})$ & 119.7 & $\mathrm{C}(8 \mathrm{~B})-\mathrm{C}(9 \mathrm{~B})-\mathrm{H}(9 \mathrm{~B})$ & 119.7 \\
\hline $\mathrm{C}(1 \mathrm{~B})-\mathrm{C}(10 \mathrm{~B})-\mathrm{H}(10 \mathrm{D})$ & 109.5 & $\mathrm{C}(1 \mathrm{~B})-\mathrm{C}(10 \mathrm{~B})-\mathrm{H}(10 \mathrm{E})$ & 109.5 \\
\hline $\mathrm{H}(10 \mathrm{D})-\mathrm{C}(10 \mathrm{~B})-\mathrm{H}(10 \mathrm{E})$ & 109.5 & $\mathrm{C}(1 \mathrm{~B})-\mathrm{C}(10 \mathrm{~B})-\mathrm{H}(10 \mathrm{~F})$ & 109.5 \\
\hline $\mathrm{H}(10 \mathrm{D})-\mathrm{C}(10 \mathrm{~B})-\mathrm{H}(10 \mathrm{~F})$ & 109.5 & $\mathrm{H}(10 \mathrm{E})-\mathrm{C}(10 \mathrm{~B})-\mathrm{H}(10 \mathrm{~F})$ & 109.5 \\
\hline $\mathrm{O}(2 \mathrm{~B})-\mathrm{C}(11 \mathrm{~B})-\mathrm{O}(3 \mathrm{~B})$ & $125.0(2)$ & $\mathrm{O}(2 \mathrm{~B})-\mathrm{C}(11 \mathrm{~B})-\mathrm{C}(1 \mathrm{~B})$ & $122.4(2)$ \\
\hline $\mathrm{O}(3 \mathrm{~B})-\mathrm{C}(11 \mathrm{~B})-\mathrm{C}(1 \mathrm{~B})$ & $112.63(18)$ & & \\
\hline $\mathrm{C}(1 \mathrm{C})-\mathrm{O}(1 \mathrm{C})-\mathrm{H}(1 \mathrm{C})$ & 109.5 & $\mathrm{C}(11 \mathrm{C})-\mathrm{O}(3 \mathrm{C})-\mathrm{H}(3 \mathrm{C})$ & 109.5 \\
\hline
\end{tabular}




$\begin{array}{llll}\mathrm{O}(1 \mathrm{C})-\mathrm{C}(1 \mathrm{C})-\mathrm{C}(2 \mathrm{C}) & 108.94(19) & \mathrm{O}(1 \mathrm{C})-\mathrm{C}(1 \mathrm{C})-\mathrm{C}(11 \mathrm{C}) & 108.53(18) \\ \mathrm{C}(2 \mathrm{C})-\mathrm{C}(1 \mathrm{C})-\mathrm{C}(11 \mathrm{C}) & 111.86(19) & \mathrm{O}(1 \mathrm{C})-\mathrm{C}(1 \mathrm{C})-\mathrm{C}(10 \mathrm{C}) & 110.51(19) \\ \mathrm{C}(2 \mathrm{C})-\mathrm{C}(1 \mathrm{C})-\mathrm{C}(10 \mathrm{C}) & 109.44(19) & \mathrm{C}(11 \mathrm{C})-\mathrm{C}(1 \mathrm{C})-\mathrm{C}(10 \mathrm{C}) & 107.56(19) \\ \mathrm{C}(3 \mathrm{C})-\mathrm{C}(2 \mathrm{C})-\mathrm{C}(1 \mathrm{C}) & 123.1(2) & \mathrm{C}(3 \mathrm{C})-\mathrm{C}(2 \mathrm{C})-\mathrm{H}(2 \mathrm{C}) & 118.4 \\ \mathrm{C}(1 \mathrm{C})-\mathrm{C}(2 \mathrm{C})-\mathrm{H}(2 \mathrm{C}) & 118.4 & \mathrm{C}(2 \mathrm{C})-\mathrm{C}(3 \mathrm{C})-\mathrm{C}(4 \mathrm{C}) & 126.8(2) \\ \mathrm{C}(2 \mathrm{C})-\mathrm{C}(3 \mathrm{C})-\mathrm{H}(3 \mathrm{C} 1) & 116.6 & \mathrm{C}(4 \mathrm{C})-\mathrm{C}(3 \mathrm{C})-\mathrm{H}(3 \mathrm{C} 1) & 116.6 \\ \mathrm{C}(9 \mathrm{C})-\mathrm{C}(4 \mathrm{C})-\mathrm{C}(5 \mathrm{C}) & 118.9(2) & \mathrm{C}(9 \mathrm{C})-\mathrm{C}(4 \mathrm{C})-\mathrm{C}(3 \mathrm{C}) & 117.8(2) \\ \mathrm{C}(5 \mathrm{C})-\mathrm{C}(4 \mathrm{C})-\mathrm{C}(3 \mathrm{C}) & 123.3(2) & \mathrm{C}(6 \mathrm{C})-\mathrm{C}(5 \mathrm{C})-\mathrm{C}(4 \mathrm{C}) & 120.1(2) \\ \mathrm{C}(6 \mathrm{C})-\mathrm{C}(5 \mathrm{C})-\mathrm{H}(5 \mathrm{C}) & 119.9 & \mathrm{C}(4 \mathrm{C})-\mathrm{C}(5 \mathrm{C})-\mathrm{H}(5 \mathrm{C}) & 119.9 \\ \mathrm{C}(7 \mathrm{C})-\mathrm{C}(6 \mathrm{C})-\mathrm{C}(5 \mathrm{C}) & 120.5(2) & \mathrm{C}(7 \mathrm{C})-\mathrm{C}(6 \mathrm{C})-\mathrm{H}(6 \mathrm{C}) & 119.7 \\ \mathrm{C}(5 \mathrm{C})-\mathrm{C}(6 \mathrm{C})-\mathrm{H}(6 \mathrm{C}) & 119.7 & \mathrm{C}(6 \mathrm{C})-\mathrm{C}(7 \mathrm{C})-\mathrm{C}(8 \mathrm{C}) & 119.7(2) \\ \mathrm{C}(6 \mathrm{C})-\mathrm{C}(7 \mathrm{C})-\mathrm{H}(7 \mathrm{C}) & 120.1 & \mathrm{C}(8 \mathrm{C})-\mathrm{C}(7 \mathrm{C})-\mathrm{H}(7 \mathrm{C}) & 120.1 \\ \mathrm{C}(7 \mathrm{C})-\mathrm{C}(8 \mathrm{C})-\mathrm{C}(9 \mathrm{C}) & 120.0(2) & \mathrm{C}(7 \mathrm{C})-\mathrm{C}(8 \mathrm{C})-\mathrm{H}(8 \mathrm{C}) & 120.0 \\ \mathrm{C}(9 \mathrm{C})-\mathrm{C}(8 \mathrm{C})-\mathrm{H}(8 \mathrm{C}) & 120.0 & \mathrm{C}(8 \mathrm{C})-\mathrm{C}(9 \mathrm{C})-\mathrm{C}(4 \mathrm{C}) & 120.7(2) \\ \mathrm{C}(8 \mathrm{C})-\mathrm{C}(9 \mathrm{C})-\mathrm{H}(9 \mathrm{C}) & 119.7 & \mathrm{C}(4 \mathrm{C})-\mathrm{C}(9 \mathrm{C})-\mathrm{H}(9 \mathrm{C}) & 119.7 \\ \mathrm{C}(1 \mathrm{C})-\mathrm{C}(10 \mathrm{C})-\mathrm{H}(10 \mathrm{G}) & 109.5 & \mathrm{C}(1 \mathrm{C})-\mathrm{C}(10 \mathrm{C})-\mathrm{H}(10 \mathrm{H}) & 109.5 \\ \mathrm{H}(10 \mathrm{G})-\mathrm{C}(10 \mathrm{C})-\mathrm{H}(10 \mathrm{H}) & 109.5 & \mathrm{C}(1 \mathrm{C})-\mathrm{C}(10 \mathrm{C})-\mathrm{H}(10 \mathrm{I}) & 109.5 \\ \mathrm{H}(10 \mathrm{G})-\mathrm{C}(10 \mathrm{C})-\mathrm{H}(10 \mathrm{I}) & 109.5 & \mathrm{H}(10 \mathrm{H})-\mathrm{C}(10 \mathrm{C})-\mathrm{H}(10 \mathrm{I}) & 109.5 \\ \mathrm{O}(2 \mathrm{C})-\mathrm{C}(11 \mathrm{C})-\mathrm{O}(3 \mathrm{C}) & 125.1(2) & \mathrm{O}(2 \mathrm{C})-\mathrm{C}(11 \mathrm{C})-\mathrm{C}(1 \mathrm{C}) & 122.3(2) \\ \mathrm{O}(3 \mathrm{C})-\mathrm{C}(11 \mathrm{C})-\mathrm{C}(1 \mathrm{C}) & 112.53(18) & & \\ & & & \end{array}$


Table S5. Anisotropic displacement parameters $\left(\AA^{2} \times 10^{3}\right)$ for $4498 . \quad$ The anisotropic displacement factor exponent takes the form: $-2 \pi^{2}\left[h^{2} a^{* 2} U^{11}+\ldots+2 h k a^{*} b^{*} U^{12}\right]$

\begin{tabular}{|c|c|c|c|c|c|c|}
\hline & $\mathrm{U}^{11}$ & $\mathrm{U}^{22}$ & $\mathrm{U}^{33}$ & $\mathrm{U}^{23}$ & $\mathrm{U}^{13}$ & $\mathrm{U}^{12}$ \\
\hline $\mathrm{O}(1 \mathrm{~A})$ & $12(1)$ & $22(1)$ & $17(1)$ & $-3(1)$ & $1(1)$ & $0(1)$ \\
\hline $\mathrm{O}(2 \mathrm{~A})$ & $18(1)$ & $28(1)$ & $36(1)$ & $-14(1)$ & $-2(1)$ & $-2(1)$ \\
\hline $\mathrm{O}(3 \mathrm{~A})$ & $10(1)$ & $28(1)$ & $22(1)$ & $-5(1)$ & $1(1)$ & $-2(1)$ \\
\hline$C(1 \mathrm{~A})$ & $10(1)$ & $21(1)$ & $14(1)$ & $-1(1)$ & $1(1)$ & $1(1)$ \\
\hline$C(2 A)$ & $15(1)$ & $18(1)$ & $13(1)$ & $-1(1)$ & $1(1)$ & $-2(1)$ \\
\hline$C(3 A)$ & $16(1)$ & $17(1)$ & $16(1)$ & $-1(1)$ & $1(1)$ & $-2(1)$ \\
\hline$C(4 A)$ & $17(1)$ & $15(1)$ & $15(1)$ & $3(1)$ & $-1(1)$ & $-2(1)$ \\
\hline$C(5 A)$ & $16(1)$ & $17(1)$ & $18(1)$ & $3(1)$ & $-2(1)$ & $0(1)$ \\
\hline$C(6 A)$ & $23(2)$ & 19(1) & $16(1)$ & $1(1)$ & $3(1)$ & $0(1)$ \\
\hline $\mathrm{C}(7 \mathrm{~A})$ & $25(1)$ & $19(1)$ & $15(1)$ & $4(1)$ & $-3(1)$ & $-1(1)$ \\
\hline $\mathrm{C}(8 \mathrm{~A})$ & $18(1)$ & $20(1)$ & $20(1)$ & $4(1)$ & $-4(1)$ & $2(1)$ \\
\hline $\mathrm{C}(9 \mathrm{~A})$ & $20(1)$ & $20(1)$ & $17(1)$ & $1(1)$ & $3(1)$ & $0(1)$ \\
\hline$C(10 A)$ & $15(1)$ & $24(1)$ & $14(1)$ & $0(1)$ & $-2(1)$ & $-1(1)$ \\
\hline \multirow[t]{2}{*}{$\mathrm{C}(11 \mathrm{~A})$} & $14(1)$ & $20(1)$ & $14(1)$ & $3(1)$ & $0(1)$ & $0(1)$ \\
\hline & $\mathrm{U}^{11}$ & $\mathrm{U}^{22}$ & $\mathrm{U}^{33}$ & $\mathrm{U}^{23}$ & $\mathrm{U}^{13}$ & $\mathrm{U}^{12}$ \\
\hline $\mathrm{O}(1 \mathrm{~B})$ & $10(1)$ & $31(1)$ & 11(1) & $-7(1)$ & $-1(1)$ & $2(1)$ \\
\hline $\mathrm{O}(2 \mathrm{~B})$ & $16(1)$ & $32(1)$ & $14(1)$ & $-8(1)$ & $-2(1)$ & $-1(1)$ \\
\hline $\mathrm{O}(3 \mathrm{~B})$ & $10(1)$ & $29(1)$ & $18(1)$ & $-5(1)$ & $-1(1)$ & $0(1)$ \\
\hline$C(1 B)$ & $10(1)$ & $22(1)$ & 11(1) & $-2(1)$ & $0(1)$ & $0(1)$ \\
\hline $\mathrm{C}(2 \mathrm{~B})$ & $12(1)$ & $18(1)$ & $12(1)$ & $-4(1)$ & $0(1)$ & $-1(1)$ \\
\hline$C(3 B)$ & $14(1)$ & $18(1)$ & $14(1)$ & $-3(1)$ & $1(1)$ & $-1(1)$ \\
\hline$C(4 B)$ & $19(1)$ & $14(1)$ & $14(1)$ & $0(1)$ & $-1(1)$ & $-3(1)$ \\
\hline $\mathrm{C}(5 \mathrm{~B})$ & $19(1)$ & $21(1)$ & $14(1)$ & $2(1)$ & $-1(1)$ & $0(1)$ \\
\hline$C(6 B)$ & $31(2)$ & $24(1)$ & $13(1)$ & $-2(1)$ & $6(1)$ & $-1(1)$ \\
\hline $\mathrm{C}(7 \mathrm{~B})$ & $36(2)$ & $28(1)$ & $12(1)$ & $4(1)$ & $-4(1)$ & $-4(1)$ \\
\hline $\mathrm{C}(8 \mathrm{~B})$ & $27(2)$ & $30(1)$ & $18(1)$ & $7(1)$ & $-7(1)$ & $1(1)$ \\
\hline $\mathrm{C}(9 \mathrm{~B})$ & $23(2)$ & $21(1)$ & $16(1)$ & $1(1)$ & $-1(1)$ & $2(1)$ \\
\hline
\end{tabular}




\begin{tabular}{|c|c|c|c|c|c|c|c|}
\hline $\mathrm{C}(10 \mathrm{~B})$ & $20(1)$ & $26(1)$ & $15(1)$ & & $4(1)$ & $-1(1)$ & $-3(1)$ \\
\hline \multirow[t]{2}{*}{$\mathrm{C}(11 \mathrm{~B})$} & $14(1)$ & $21(1)$ & $8(1)$ & & $3(1)$ & $0(1)$ & $-1(1)$ \\
\hline & $\mathrm{U}^{11}$ & $\mathrm{U}^{22}$ & $\mathrm{U}^{33}$ & & $\mathrm{U}^{23}$ & $\mathrm{U}^{13}$ & $\mathrm{U}^{12}$ \\
\hline $\mathrm{O}(1 \mathrm{C})$ & $11(1)$ & $31(1)$ & $13(1)$ & & $-8(1)$ & $1(1)$ & $-2(1)$ \\
\hline $\mathrm{O}(2 \mathrm{C})$ & $16(1)$ & $29(1)$ & $15(1)$ & & $-7(1)$ & $2(1)$ & $1(1)$ \\
\hline $\mathrm{O}(3 \mathrm{C})$ & $9(1)$ & $28(1)$ & $23(1)$ & & $-6(1)$ & $0(1)$ & $1(1)$ \\
\hline $\mathrm{C}(1 \mathrm{C})$ & $9(1)$ & $24(1)$ & $12(1)$ & & $-2(1)$ & $-1(1)$ & $-2(1)$ \\
\hline $\mathrm{C}(2 \mathrm{C})$ & $12(1)$ & $21(1)$ & 11(1) & & $-4(1)$ & $-1(1)$ & $4(1)$ \\
\hline $\mathrm{C}(3 \mathrm{C})$ & $15(1)$ & $18(1)$ & $12(1)$ & & $-4(1)$ & $-2(1)$ & $2(1)$ \\
\hline $\mathrm{C}(4 \mathrm{C})$ & $18(1)$ & $15(1)$ & 11(1) & & $1(1)$ & $0(1)$ & $2(1)$ \\
\hline $\mathrm{C}(5 \mathrm{C})$ & $14(1)$ & $20(1)$ & $14(1)$ & & $2(1)$ & $1(1)$ & $1(1)$ \\
\hline$C(6 C)$ & $25(2)$ & 19(1) & $13(1)$ & & $-1(1)$ & $-2(1)$ & $-1(1)$ \\
\hline$C(7 C)$ & $26(2)$ & 19(1) & $10(1)$ & & $2(1)$ & $2(1)$ & $1(1)$ \\
\hline $\mathrm{C}(8 \mathrm{C})$ & $20(1)$ & $24(1)$ & $16(1)$ & & $3(1)$ & $5(1)$ & $-1(1)$ \\
\hline $\mathrm{C}(9 \mathrm{C})$ & $18(1)$ & $21(1)$ & $13(1)$ & & $0(1)$ & $-2(1)$ & $-1(1)$ \\
\hline $\mathrm{C}(10 \mathrm{C})$ & $18(1)$ & $28(1)$ & $13(1)$ & & $1(1)$ & $0(1)$ & $4(1)$ \\
\hline $\mathrm{C}(11 \mathrm{C})$ & $13(1)$ & $24(1)$ & $8(1)$ & & $1(1)$ & $0(1)$ & $0(1)$ \\
\hline \multirow{2}{*}{$\begin{array}{l}\text { Table S6. } \\
\text { for } 4498 .\end{array}$} & \multicolumn{7}{|c|}{ Hydrogen coordinates $\left(x 1^{4}\right)$ and isotropic displacement parameters $\left(\AA^{2} \times 10^{3}\right.$} \\
\hline & & $\mathrm{x}$ & \multicolumn{3}{|c|}{$\mathrm{y}$} & $\mathrm{z}$ & $\mathrm{U}(\mathrm{eq})$ \\
\hline $\mathrm{H}(1 \mathrm{~A})$ & & 7082 & \multicolumn{2}{|r|}{2934} & \multicolumn{2}{|r|}{5157} & 26 \\
\hline $\mathrm{H}(3 \mathrm{~A})$ & & 341 & \multicolumn{2}{|r|}{3526} & \multicolumn{2}{|r|}{5327} & 30 \\
\hline $\mathrm{H}(2 \mathrm{~A})$ & & 4567 & \multicolumn{2}{|r|}{3770} & \multicolumn{2}{|r|}{6337} & 19 \\
\hline $\mathrm{H}(3 \mathrm{~A} 1)$ & & 8269 & \multicolumn{2}{|r|}{2990} & \multicolumn{2}{|r|}{6164} & 20 \\
\hline \multirow[t]{2}{*}{$\mathrm{H}(5 \mathrm{~A})$} & & 4471 & \multicolumn{2}{|r|}{3548} & \multicolumn{2}{|r|}{7277} & 21 \\
\hline & $\mathrm{U}^{11}$ & $\mathrm{U}^{22}$ & $\mathrm{U}^{33}$ & & $\mathrm{U}^{23}$ & $\mathrm{U}^{13}$ & $\mathrm{U}^{12}$ \\
\hline
\end{tabular}




$\begin{array}{lrlll}\mathrm{H}(6 \mathrm{~A}) & 5185 & 3515 & 8269 & 23 \\ \mathrm{H}(7 \mathrm{~A}) & 8706 & 3159 & 8615 & 24 \\ \mathrm{H}(8 \mathrm{~A}) & 11456 & 2789 & 7968 & 24 \\ \mathrm{H}(9 \mathrm{~A}) & 10736 & 2810 & 6978 & 23 \\ \mathrm{H}(10 \mathrm{~A}) & 7317 & 4472 & 5433 & 26 \\ \mathrm{H}(10 \mathrm{~B}) & 4597 & 4553 & 5473 & 26 \\ \mathrm{H}(10 \mathrm{C}) & 5759 & 4353 & 4876 & 26\end{array}$

\begin{tabular}{|c|c|c|c|c|c|c|}
\hline & $\mathrm{U}^{11}$ & $\mathrm{U}^{22}$ & $\mathrm{U}^{33}$ & $\mathrm{U}^{23}$ & $\mathrm{U}^{13}$ & $\mathrm{U}^{12}$ \\
\hline $\mathrm{H}(1 \mathrm{~B})$ & & 7174 & 6315 & & 5270 & 25 \\
\hline $\mathrm{H}(3 \mathrm{~B})$ & & 442 & 6682 & & 5716 & 28 \\
\hline $\mathrm{H}(2 \mathrm{~B})$ & & 4672 & 7065 & & 6667 & 17 \\
\hline $\mathrm{H}(3 \mathrm{~B} 1)$ & & 8289 & 6240 & & 6587 & 18 \\
\hline $\mathrm{H}(5 \mathrm{~B})$ & & 4324 & 6914 & & 7608 & 21 \\
\hline $\mathrm{H}(6 \mathrm{~B})$ & & 4837 & 6954 & & 8608 & 27 \\
\hline $\mathrm{H}(7 \mathrm{~B})$ & & 8205 & 6582 & & 9025 & 30 \\
\hline $\mathrm{H}(8 \mathrm{~B})$ & & 11010 & 6133 & & 8447 & 30 \\
\hline $\mathrm{H}(9 \mathrm{~B})$ & & 10537 & 6092 & & 7446 & 24 \\
\hline$H(10 D)$ & & 7457 & 7649 & & 5705 & 31 \\
\hline $\mathrm{H}(10 \mathrm{E})$ & & 4742 & 7742 & & 5738 & 31 \\
\hline $\mathrm{H}(10 \mathrm{~F})$ & & 5888 & 7475 & & 5163 & 31 \\
\hline
\end{tabular}

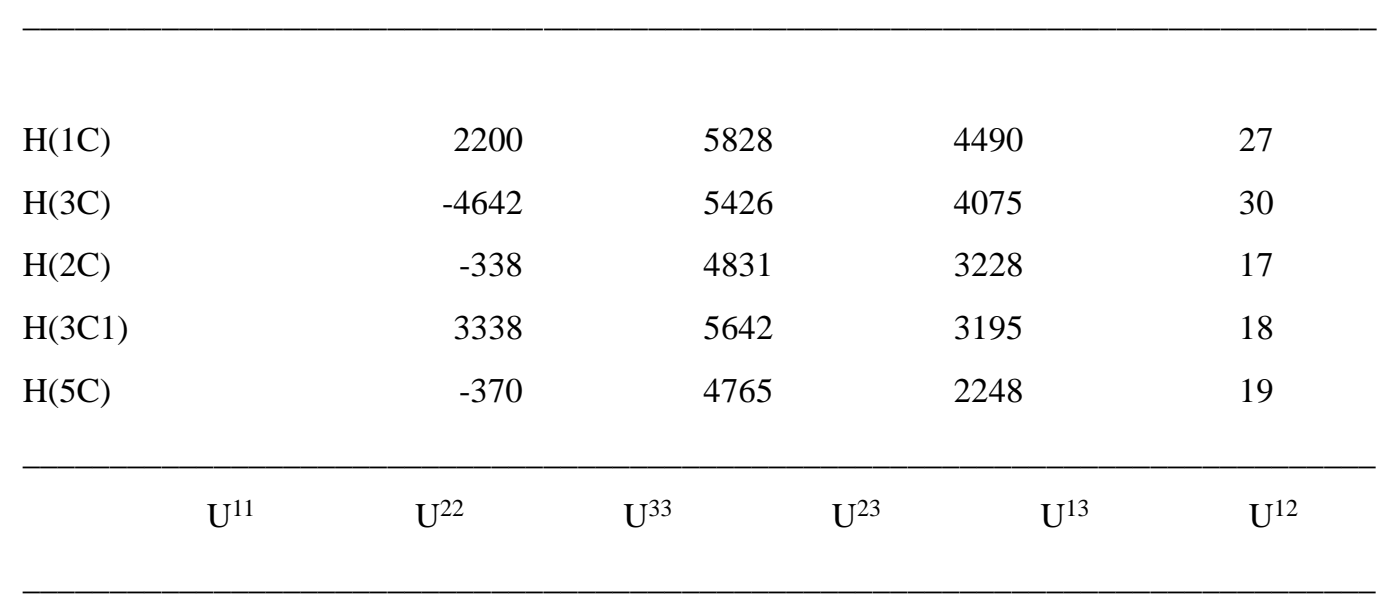

$\begin{array}{rrrrr}\mathrm{H}(6 \mathrm{C}) & 436 & 4516 & 1288 & 23 \\ \mathrm{H}(7 \mathrm{C}) & 3976 & 4766 & 886 & 22\end{array}$




$\begin{array}{lcccc}\mathrm{H}(8 \mathrm{C}) & 6701 & 5294 & 1437 & 24 \\ \mathrm{H}(9 \mathrm{C}) & 5880 & 5568 & 2390 & 21 \\ \mathrm{H}(10 \mathrm{G}) & 2325 & 4467 & 4284 & 30 \\ \mathrm{H}(10 \mathrm{H}) & -399 & 4386 & 4281 & 30 \\ \mathrm{H}(10 \mathrm{I}) & 817 & 4769 & 4786 & 30\end{array}$


Table S8. Torsion angles $\left[^{\circ}\right]$ for $f 498$.

\begin{tabular}{|c|c|}
\hline $\mathrm{O}(1 \mathrm{~A})-\mathrm{C}(1 \mathrm{~A})-\mathrm{C}(2 \mathrm{~A})-\mathrm{C}(3 \mathrm{~A})$ & $-1.5(3)$ \\
\hline$C(10 A)-C(1 A)-C(2 A)-C(3 A)$ & $-120.1(3)$ \\
\hline$C(11 \mathrm{~A})-\mathrm{C}(1 \mathrm{~A})-\mathrm{C}(2 \mathrm{~A})-\mathrm{C}(3 \mathrm{~A})$ & $118.6(3)$ \\
\hline$C(1 A)-C(2 A)-C(3 A)-C(4 A)$ & $173.9(2)$ \\
\hline$C(2 A)-C(3 A)-C(4 A)-C(5 A)$ & $15.1(4)$ \\
\hline$C(2 A)-C(3 A)-C(4 A)-C(9 A)$ & $-162.8(2)$ \\
\hline $\mathrm{C}(9 \mathrm{~A})-\mathrm{C}(4 \mathrm{~A})-\mathrm{C}(5 \mathrm{~A})-\mathrm{C}(6 \mathrm{~A})$ & $1.1(3)$ \\
\hline$C(3 A)-C(4 A)-C(5 A)-C(6 A)$ & $-176.7(2)$ \\
\hline$C(4 A)-C(5 A)-C(6 A)-C(7 A)$ & $0.4(4)$ \\
\hline$C(5 A)-C(6 A)-C(7 A)-C(8 A)$ & $-1.6(4)$ \\
\hline $\mathrm{C}(6 \mathrm{~A})-\mathrm{C}(7 \mathrm{~A})-\mathrm{C}(8 \mathrm{~A})-\mathrm{C}(9 \mathrm{~A})$ & $1.2(4)$ \\
\hline $\mathrm{C}(7 \mathrm{~A})-\mathrm{C}(8 \mathrm{~A})-\mathrm{C}(9 \mathrm{~A})-\mathrm{C}(4 \mathrm{~A})$ & $0.3(4)$ \\
\hline $\mathrm{C}(5 \mathrm{~A})-\mathrm{C}(4 \mathrm{~A})-\mathrm{C}(9 \mathrm{~A})-\mathrm{C}(8 \mathrm{~A})$ & $-1.5(4)$ \\
\hline$C(3 A)-C(4 A)-C(9 A)-C(8 A)$ & $176.5(2)$ \\
\hline$O(1 A)-C(1 A)-C(11 A)-O(2 A)$ & $6.2(3)$ \\
\hline $\mathrm{C}(2 \mathrm{~A})-\mathrm{C}(1 \mathrm{~A})-\mathrm{C}(11 \mathrm{~A})-\mathrm{O}(2 \mathrm{~A})$ & $-116.5(2)$ \\
\hline $\mathrm{C}(10 \mathrm{~A})-\mathrm{C}(1 \mathrm{~A})-\mathrm{C}(11 \mathrm{~A})-\mathrm{O}(2 \mathrm{~A})$ & $122.4(2)$ \\
\hline $\mathrm{O}(1 \mathrm{~A})-\mathrm{C}(1 \mathrm{~A})-\mathrm{C}(11 \mathrm{~A})-\mathrm{O}(3 \mathrm{~A})$ & $-174.17(18)$ \\
\hline $\mathrm{C}(2 \mathrm{~A})-\mathrm{C}(1 \mathrm{~A})-\mathrm{C}(11 \mathrm{~A})-\mathrm{O}(3 \mathrm{~A})$ & $63.2(2)$ \\
\hline$C(10 A)-C(1 A)-C(11 A)-O(3 A)$ & $-57.9(2)$ \\
\hline $\mathrm{O}(1 \mathrm{~B})-\mathrm{C}(1 \mathrm{~B})-\mathrm{C}(2 \mathrm{~B})-\mathrm{C}(3 \mathrm{~B})$ & $-1.0(3)$ \\
\hline $\mathrm{C}(11 \mathrm{~B})-\mathrm{C}(1 \mathrm{~B})-\mathrm{C}(2 \mathrm{~B})-\mathrm{C}(3 \mathrm{~B})$ & $117.9(3)$ \\
\hline $\mathrm{C}(10 \mathrm{~B})-\mathrm{C}(1 \mathrm{~B})-\mathrm{C}(2 \mathrm{~B})-\mathrm{C}(3 \mathrm{~B})$ & $-122.6(3)$ \\
\hline $\mathrm{C}(1 \mathrm{~B})-\mathrm{C}(2 \mathrm{~B})-\mathrm{C}(3 \mathrm{~B})-\mathrm{C}(4 \mathrm{~B})$ & $177.3(2)$ \\
\hline $\mathrm{C}(2 \mathrm{~B})-\mathrm{C}(3 \mathrm{~B})-\mathrm{C}(4 \mathrm{~B})-\mathrm{C}(9 \mathrm{~B})$ & $-164.3(2)$ \\
\hline $\mathrm{C}(2 \mathrm{~B})-\mathrm{C}(3 \mathrm{~B})-\mathrm{C}(4 \mathrm{~B})-\mathrm{C}(5 \mathrm{~B})$ & $14.0(4)$ \\
\hline $\mathrm{C}(9 \mathrm{~B})-\mathrm{C}(4 \mathrm{~B})-\mathrm{C}(5 \mathrm{~B})-\mathrm{C}(6 \mathrm{~B})$ & $1.5(4)$ \\
\hline $\mathrm{C}(3 \mathrm{~B})-\mathrm{C}(4 \mathrm{~B})-\mathrm{C}(5 \mathrm{~B})-\mathrm{C}(6 \mathrm{~B})$ & $-176.8(2)$ \\
\hline $\mathrm{C}(4 \mathrm{~B})-\mathrm{C}(5 \mathrm{~B})-\mathrm{C}(6 \mathrm{~B})-\mathrm{C}(7 \mathrm{~B})$ & $-0.2(4)$ \\
\hline $\mathrm{C}(5 \mathrm{~B})-\mathrm{C}(6 \mathrm{~B})-\mathrm{C}(7 \mathrm{~B})-\mathrm{C}(8 \mathrm{~B})$ & $-1.3(4)$ \\
\hline $\mathrm{C}(6 \mathrm{~B})-\mathrm{C}(7 \mathrm{~B})-\mathrm{C}(8 \mathrm{~B})-\mathrm{C}(9 \mathrm{~B})$ & $1.5(4)$ \\
\hline $\mathrm{C}(5 \mathrm{~B})-\mathrm{C}(4 \mathrm{~B})-\mathrm{C}(9 \mathrm{~B})-\mathrm{C}(8 \mathrm{~B})$ & $-1.4(4)$ \\
\hline B) $-\mathrm{C}(4 \mathrm{~B})-\mathrm{C}(9 \mathrm{~B})-\mathrm{C}(8 \mathrm{~B})$ & $177.0(2$ \\
\hline
\end{tabular}




$\begin{array}{lc}\mathrm{C}(7 \mathrm{~B})-\mathrm{C}(8 \mathrm{~B})-\mathrm{C}(9 \mathrm{~B})-\mathrm{C}(4 \mathrm{~B}) & -0.1(4) \\ \mathrm{O}(1 \mathrm{~B})-\mathrm{C}(1 \mathrm{~B})-\mathrm{C}(11 \mathrm{~B})-\mathrm{O}(2 \mathrm{~B}) & -10.6(3) \\ \mathrm{C}(2 \mathrm{~B})-\mathrm{C}(1 \mathrm{~B})-\mathrm{C}(11 \mathrm{~B})-\mathrm{O}(2 \mathrm{~B}) & -130.0(2) \\ \mathrm{C}(10 \mathrm{~B})-\mathrm{C}(1 \mathrm{~B})-\mathrm{C}(11 \mathrm{~B})-\mathrm{O}(2 \mathrm{~B}) & 109.1(2) \\ \mathrm{O}(1 \mathrm{~B})-\mathrm{C}(1 \mathrm{~B})-\mathrm{C}(11 \mathrm{~B})-\mathrm{O}(3 \mathrm{~B}) & 170.92(17) \\ \mathrm{C}(2 \mathrm{~B})-\mathrm{C}(1 \mathrm{~B})-\mathrm{C}(11 \mathrm{~B})-\mathrm{O}(3 \mathrm{~B}) & 51.6(3) \\ \mathrm{C}(10 \mathrm{~B})-\mathrm{C}(1 \mathrm{~B})-\mathrm{C}(11 \mathrm{~B})-\mathrm{O}(3 \mathrm{~B}) & -69.4(2) \\ & \\ \mathrm{O}(1 \mathrm{C})-\mathrm{C}(1 \mathrm{C})-\mathrm{C}(2 \mathrm{C})-\mathrm{C}(3 \mathrm{C}) & 0.2(3) \\ \mathrm{C}(11 \mathrm{C})-\mathrm{C}(1 \mathrm{C})-\mathrm{C}(2 \mathrm{C})-\mathrm{C}(3 \mathrm{C}) & 120.2(3) \\ \mathrm{C}(10 \mathrm{C})-\mathrm{C}(1 \mathrm{C})-\mathrm{C}(2 \mathrm{C})-\mathrm{C}(3 \mathrm{C}) & -120.7(2) \\ \mathrm{C}(1 \mathrm{C})-\mathrm{C}(2 \mathrm{C})-\mathrm{C}(3 \mathrm{C})-\mathrm{C}(4 \mathrm{C}) & 175.5(2) \\ \mathrm{C}(2 \mathrm{C})-\mathrm{C}(3 \mathrm{C})-\mathrm{C}(4 \mathrm{C})-\mathrm{C}(9 \mathrm{C}) & -162.9(2) \\ \mathrm{C}(2 \mathrm{C})-\mathrm{C}(3 \mathrm{C})-\mathrm{C}(4 \mathrm{C})-\mathrm{C}(5 \mathrm{C}) & 15.9(4) \\ \mathrm{C}(9 \mathrm{C})-\mathrm{C}(4 \mathrm{C})-\mathrm{C}(5 \mathrm{C})-\mathrm{C}(6 \mathrm{C}) & 0.8(3) \\ \mathrm{C}(3 \mathrm{C})-\mathrm{C}(4 \mathrm{C})-\mathrm{C}(5 \mathrm{C})-\mathrm{C}(6 \mathrm{C}) & -178.0(2) \\ \mathrm{C}(4 \mathrm{C})-\mathrm{C}(5 \mathrm{C})-\mathrm{C}(6 \mathrm{C})-\mathrm{C}(7 \mathrm{C}) & 0.5(3) \\ \mathrm{C}(5 \mathrm{C})-\mathrm{C}(6 \mathrm{C})-\mathrm{C}(7 \mathrm{C})-\mathrm{C}(8 \mathrm{C}) & -1.0(4) \\ \mathrm{C}(6 \mathrm{C})-\mathrm{C}(7 \mathrm{C})-\mathrm{C}(8 \mathrm{C})-\mathrm{C}(9 \mathrm{C}) & 0.1(4) \\ \mathrm{C}(7 \mathrm{C})-\mathrm{C}(8 \mathrm{C})-\mathrm{C}(9 \mathrm{C})-\mathrm{C}(4 \mathrm{C}) & 1.2(4) \\ \mathrm{C}(5 \mathrm{C})-\mathrm{C}(4 \mathrm{C})-\mathrm{C}(9 \mathrm{C})-\mathrm{C}(8 \mathrm{C}) & -1.7(3) \\ \mathrm{C}(3 \mathrm{C})-\mathrm{C}(4 \mathrm{C})-\mathrm{C}(9 \mathrm{C})-\mathrm{C}(8 \mathrm{C}) & 177.2(2) \\ \mathrm{O}(1 \mathrm{C})-\mathrm{C}(1 \mathrm{C})-\mathrm{C}(11 \mathrm{C})-\mathrm{O}(2 \mathrm{C}) & -12.8(3) \\ \mathrm{C}(2 \mathrm{C})-\mathrm{C}(1 \mathrm{C})-\mathrm{C}(11 \mathrm{C})-\mathrm{O}(2 \mathrm{C}) & -133.0(2) \\ \mathrm{C}(10 \mathrm{C})-\mathrm{C}(1 \mathrm{C})-\mathrm{C}(11 \mathrm{C})-\mathrm{O}(2 \mathrm{C}) & 106.8(2) \\ \mathrm{O}(1 \mathrm{C})-\mathrm{C}(1 \mathrm{C})-\mathrm{C}(11 \mathrm{C})-\mathrm{O}(3 \mathrm{C}) & -70.8(2) \\ \mathrm{C}(2 \mathrm{C})-\mathrm{C}(1 \mathrm{C})-\mathrm{C}(11 \mathrm{C})-\mathrm{O}(3 \mathrm{C}) & \\ \mathrm{C}(10 \mathrm{C})-\mathrm{C}(1 \mathrm{C})-\mathrm{C}(11 \mathrm{C})-\mathrm{O}(3 \mathrm{C}) & \\ & \\ & \\ & \end{array}$


Table S9. Hydrogen bonds for 4498 [A and ${ }^{\circ}$ ].

\begin{tabular}{|c|c|c|c|c|}
\hline D-H...A & $\mathrm{d}(\mathrm{D}-\mathrm{H})$ & d(H...A) & $\mathrm{d}(\mathrm{D} \ldots \mathrm{A})$ & $<(\mathrm{DHA})$ \\
\hline $\mathrm{O}(1 \mathrm{~A})-\mathrm{H}(1 \mathrm{~A}) \ldots \mathrm{O}(2 \mathrm{~A}) \# 1$ & 0.84 & 1.98 & $2.727(3)$ & 147.7 \\
\hline $\mathrm{O}(3 \mathrm{~A})-\mathrm{H}(3 \mathrm{~A}) \ldots \mathrm{O}(1 \mathrm{~A}) \# 2$ & 0.84 & 1.81 & $2.630(3)$ & 166.4 \\
\hline $\mathrm{O}(1 \mathrm{~B})-\mathrm{H}(1 \mathrm{~B}) \ldots \mathrm{O}(2 \mathrm{C}) \# 3$ & 0.84 & 2.04 & $2.800(2)$ & 149.7 \\
\hline $\mathrm{O}(3 \mathrm{~B})-\mathrm{H}(3 \mathrm{~B}) \ldots \mathrm{O}(1 \mathrm{~B}) \# 2$ & 0.84 & 1.82 & $2.640(2)$ & 165.2 \\
\hline $\mathrm{O}(1 \mathrm{C})-\mathrm{H}(1 \mathrm{C}) \ldots \mathrm{O}(2 \mathrm{~B})$ & 0.84 & 1.99 & $2.758(2)$ & 152.7 \\
\hline $\mathrm{O}(3 \mathrm{C})-\mathrm{H}(3 \mathrm{C}) \ldots \mathrm{O}(1 \mathrm{C}) \# 2$ & 0.84 & 1.82 & $2.644(3)$ & 166.2 \\
\hline
\end{tabular}

Symmetry transformations used to generate equivalent atoms:

$\# 1 \mathrm{x}+1 / 2,-\mathrm{y}+1 / 2,-\mathrm{z}+1 \quad \# 2 \mathrm{x}-1, \mathrm{y}, \mathrm{z} \quad \# 3 \mathrm{x}+1, \mathrm{y}, \mathrm{z}$ 


\section{X-ray analysis of ent-2i}

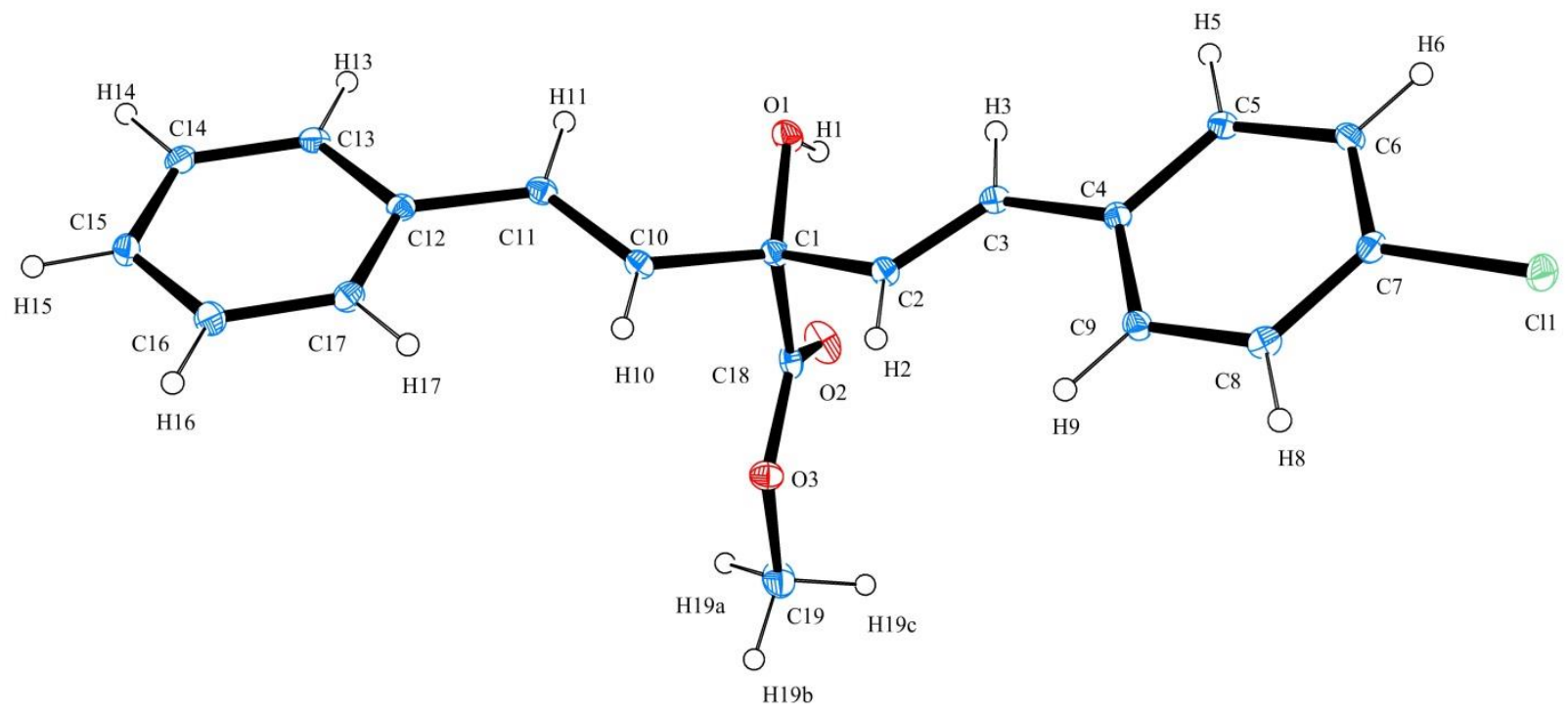

Molecule's view:

Table S1. Sample and crystal data for g061.

Identification code

Chemical formula

Formula weight

Temperature

Wavelength

Crystal size

Crystal system

Space group

Unit cell dimensions g061

$\mathrm{C}_{19} \mathrm{H}_{17} \mathrm{ClO}_{3}$

$328.77 \mathrm{~g} / \mathrm{mol}$

100(2) K

$1.54178 \AA$

0.117 x 0.157 x $0.208 \mathrm{~mm}$

monoclinic

P 12 (1) 1

$$
\begin{array}{ll}
\mathrm{a}=5.9854(7) \AA & \alpha=90^{\circ} \\
\mathrm{b}=7.5004(8) \AA & \beta=97.565(4)^{\circ}
\end{array}
$$




$$
\mathrm{c}=18.085(2) \AA \quad \gamma=90^{\circ}
$$

Volume

$\mathbf{Z}$

Density (calculated)

Absorption coefficient

$\mathbf{F}(000)$
804.82(16) $\AA^{3}$

2

$1.357 \mathrm{~g} / \mathrm{cm}^{3}$

$2.205 \mathrm{~mm}^{-1}$

344

Table S2. Data collection and structure refinement for g061.

\begin{tabular}{|c|c|}
\hline Theta range for data collection & 4.93 to $68.19^{\circ}$ \\
\hline Index ranges & $-7<=\mathrm{h}<=7,-9<=\mathrm{k}<=9,-21<=\mathrm{l}<=21$ \\
\hline Reflections collected & 10346 \\
\hline Independent reflections & $2871[\mathrm{R}(\mathrm{int})=0.0289]$ \\
\hline Coverage of independent reflections & $99.9 \%$ \\
\hline Absorption correction & multi-scan \\
\hline Max. and min. transmission & 0.7820 and 0.6570 \\
\hline Refinement method & Full-matrix least-squares on $\mathrm{F}^{2}$ \\
\hline Refinement program & SHELXL-2014/7 (Sheldrick, 2014) \\
\hline Function minimized & $\Sigma \mathrm{w}\left(\mathrm{F}_{\mathrm{o}}^{2}-\mathrm{F}_{\mathrm{c}}^{2}\right)^{2}$ \\
\hline Data / restraints / parameters & $2871 / 1 / 210$ \\
\hline Goodness-of-fit on $\mathbf{F}^{2}$ & 1.031 \\
\hline \multirow[t]{3}{*}{ Final $\mathbf{R}$ indices } & 2798 data; $\mathrm{I}>2 \sigma(\mathrm{I}) \mathrm{R} 1=0.0233$, wR $2=0.0580$ \\
\hline & $\mathrm{R} 1=0.0243, \mathrm{wR} 2=0.0587$ \\
\hline & $\mathrm{w}=1 /\left[\sigma^{2}\left(\mathrm{~F}_{\mathrm{o}}^{2}\right)+(0.0219 \mathrm{P})^{2}+0.1451 \mathrm{P}\right]$ \\
\hline Weighting scheme & where $\mathrm{P}=\left(\mathrm{F}_{\mathrm{o}}^{2}+2 \mathrm{~F}_{\mathrm{c}}^{2}\right) / 3$ \\
\hline Absolute structure parameter & $0.057(5)^{*}$ \\
\hline
\end{tabular}




\section{Largest diff. peak and hole $\quad 0.251$ and $-0.195 \mathrm{e}^{-3}$ \\ R.M.S. deviation from mean $\quad 0.037 \mathrm{e}^{-3}$}

*Flack x determined using 1213 quotients $[(\mathrm{I}+)-(\mathrm{I}-)] /[(\mathrm{I}+)+(\mathrm{I}-)]$

(Parsons, Flack and Wagner, Acta Cryst. B69 (2013) 249-259).

Table S3. Atomic coordinates and equivalent isotropic atomic displacement parameters $\left(\AA^{2}\right)$ for g061.

$\mathrm{U}(\mathrm{eq})$ is defined as one third of the trace of the orthogonalized $\mathrm{U}_{\mathrm{ij}}$ tensor.
$\mathbf{x} / \mathbf{a}$
$\mathbf{y} / \mathbf{b}$
$\mathbf{z} / \mathbf{c}$
$\mathbf{U}(\mathbf{e q})$
Cl1 $0.79884(7) \quad 0.55602(7) \quad 0.33395(3) \quad 0.02424(14)$
O3 $0.4577(2) \quad 0.71188(19) \quad 0.80350(8) \quad 0.0216(3)$
$\mathrm{O} 1 \quad 0.9135(2) \quad 0.6798(2) \quad 0.69514(9) \quad 0.0220(3)$
O2 $0.6948(3) \quad 0.9185(2) \quad 0.76827(9) \quad 0.0299(4)$
C5 $0.3867(3) \quad 0.6556(3) \quad 0.45817(11) \quad 0.0163(4)$
C6 $0.2199(3) \quad 0.6417(3) \quad 0.39750(12) \quad 0.0183(4)$
C9 $0.1440(3) \quad 0.5199(3) \quad 0.53778(12) \quad 0.0173(4)$
C18 0.6259(3) $\quad 0.7682(3) \quad 0.76763(11) \quad 0.0193(4)$ 


$\begin{array}{cccccc} & \mathbf{x} / \mathbf{a} & \mathbf{y} / \mathbf{b} & \mathbf{z} / \mathbf{c} & \mathbf{U}(\mathbf{e q}) \\ \mathrm{C} 4 & 0.3530(3) & 0.5967(2) & 0.52911(11) & 0.0152(4) \\ \mathrm{C} 8 & 0.9767(3) & 0.5042(3) & 0.47791(12) & 0.0184(4) \\ \mathrm{C} 7 & 0.0153(3) & 0.5671(3) & 0.40846(10) & 0.0174(4) \\ \mathrm{C} 11 & 0.9936(3) & 0.4317(3) & 0.80769(12) & 0.0179(4) \\ \mathrm{C} 10 & 0.7830(3) & 0.4633(3) & 0.77727(11) & 0.0170(4) \\ \mathrm{C} 3 & 0.5331(3) & 0.6229(3) & 0.59158(12) & 0.0165(4) \\ \mathrm{C} 2 & 0.5330(3) & 0.5682(3) & 0.66146(10) & 0.0165(4) \\ \mathrm{C} 14 & 0.3631(3) & 0.2144(3) & 0.96617(12) & 0.0198(4) \\ \mathrm{C} 13 & 0.2891(3) & 0.3181(3) & 0.90420(12) & 0.0182(4) \\ \mathrm{C} 12 & 0.0672(3) & 0.3069(3) & 0.86849(11) & 0.0165(4) \\ \mathrm{C} 17 & 0.9231(3) & 0.1841(3) & 0.89625(12) & 0.0186(4) \\ \mathrm{C} 1 & 0.7198(3) & 0.6156(3) & 0.72358(11) & 0.0179(4) \\ \mathrm{C} 16 & 0.9963(3) & 0.0814(3) & 0.95847(12) & 0.0218(4) \\ \mathrm{C} 15 & 0.2156(3) & 0.0976(3) & 0.99432(12) & 0.0210(4) \\ \mathrm{C} 19 & 0.3565(4) & 0.8446(3) & 0.84700(13) & 0.0295(5)\end{array}$

Table S4. Bond lengths (Å) for g061.

$\begin{array}{llll}\text { C11-C7 } & 1.7439(19) & \text { O3-C18 } & 1.336(3) \\ \text { O3-C19 } & 1.450(3) & \text { O1-C1 } & 1.413(2) \\ \text { O1-H1 } & 0.84 & \text { O2-C18 } & 1.199(3)\end{array}$




\begin{tabular}{llll} 
C5-C6 & $1.387(3)$ & C5-C4 & $1.397(3)$ \\
C5-H5 & 0.95 & C6-C7 & $1.384(3)$ \\
C6-H6 & 0.95 & C9-C8 & $1.380(3)$ \\
C9-C4 & $1.404(3)$ & C9-H9 & 0.95 \\
C18-C1 & $1.542(3)$ & C4-C3 & $1.468(3)$ \\
C8-C7 & $1.389(3)$ & C8-H8 & 0.95 \\
C11-C10 & $1.329(3)$ & C11-C12 & $1.467(3)$ \\
C11-H11 & 0.95 & C10-C1 & $1.515(3)$ \\
C10-H10 & 0.95 & C3-C2 & $1.329(3)$ \\
C3-H3 & 0.95 & C2-C1 & $1.519(3)$ \\
C2-H2 & 0.95 & C14-C15 & $1.387(3)$ \\
C14-C13 & $1.388(3)$ & C14-H14 & 0.95 \\
C13-C12 & $1.401(3)$ & C13-H13 & 0.95 \\
C12-C17 & $1.400(3)$ & C17-C16 & $1.386(3)$ \\
C17-H17 & 0.95 & C16-C15 & $1.391(3)$ \\
C16-H16 & 0.95 & C15-H15 & 0.95 \\
C19-H19A & 0.98 & C19-H19B & 0.98 \\
C19-H19C & 0.98 & & \\
\hline
\end{tabular}

Table S5. Bond angles $\left(^{\circ}\right)$ for g061.

$\begin{array}{llll}\text { C18-O3-C19 } & 116.21(17) & \text { C1-O1-H1 } & 109.5 \\ \text { C6-C5-C4 } & 121.81(18) & \text { C6-C5-H5 } & 119.1 \\ \text { C4-C5-H5 } & 119.1 & \text { C7-C6-C5 } & 118.39(18) \\ \text { C7-C6-H6 } & 120.8 & \text { C5-C6-H6 } & 120.8 \\ \text { C8-C9-C4 } & 120.84(18) & \text { C8-C9-H9 } & 119.6\end{array}$




\begin{tabular}{|c|c|c|c|}
\hline C4-C9-H9 & 119.6 & $\mathrm{O} 2-\mathrm{C} 18-\mathrm{O} 3$ & $124.9(2)$ \\
\hline $\mathrm{O} 2-\mathrm{C} 18-\mathrm{C} 1$ & 123.62(19) & $\mathrm{O} 3-\mathrm{C} 18-\mathrm{C} 1$ & $111.43(16)$ \\
\hline $\mathrm{C} 5-\mathrm{C} 4-\mathrm{C} 9$ & $118.09(18)$ & $\mathrm{C} 5-\mathrm{C} 4-\mathrm{C} 3$ & $118.95(17)$ \\
\hline $\mathrm{C} 9-\mathrm{C} 4-\mathrm{C} 3$ & $122.93(17)$ & C9-C8-C7 & $119.36(18)$ \\
\hline C9-C8-H8 & 120.3 & C7-C8-H8 & 120.3 \\
\hline C6-C7-C8 & $121.49(18)$ & C6-C7-Cl1 & $119.34(15)$ \\
\hline C8-C7-C11 & $119.16(15)$ & C10-C11-C12 & $126.82(18)$ \\
\hline C10-C11-H11 & 116.6 & C12-C11-H11 & 116.6 \\
\hline C11-C10-C1 & $122.88(18)$ & C11-C10-H10 & 118.6 \\
\hline C1-C10-H10 & 118.6 & $\mathrm{C} 2-\mathrm{C} 3-\mathrm{C} 4$ & $126.75(18)$ \\
\hline $\mathrm{C} 2-\mathrm{C} 3-\mathrm{H} 3$ & 116.6 & $\mathrm{C} 4-\mathrm{C} 3-\mathrm{H} 3$ & 116.6 \\
\hline $\mathrm{C} 3-\mathrm{C} 2-\mathrm{C} 1$ & 122.61(18) & $\mathrm{C} 3-\mathrm{C} 2-\mathrm{H} 2$ & 118.7 \\
\hline $\mathrm{C} 1-\mathrm{C} 2-\mathrm{H} 2$ & 118.7 & C15-C14-C13 & $119.97(19)$ \\
\hline C15-C14-H14 & 120.0 & C13-C14-H14 & 120.0 \\
\hline C14-C13-C12 & 121.39(19) & C14-C13-H13 & 119.3 \\
\hline C12-C13-H13 & 119.3 & C17-C12-C13 & $117.78(19)$ \\
\hline C17-C12-C11 & $123.38(19)$ & C13-C12-C11 & $118.71(18)$ \\
\hline C16-C17-C12 & $120.86(19)$ & C16-C17-H17 & 119.6 \\
\hline C12-C17-H17 & 119.6 & $\mathrm{O} 1-\mathrm{C} 1-\mathrm{C} 10$ & $110.06(16)$ \\
\hline $\mathrm{O} 1-\mathrm{C} 1-\mathrm{C} 2$ & $111.69(16)$ & $\mathrm{C} 10-\mathrm{C} 1-\mathrm{C} 2$ & $113.25(17)$ \\
\hline O1-C1-C18 & 107.91(16) & C10-C1-C18 & $107.81(16)$ \\
\hline C2-C1-C18 & $105.82(16)$ & C17-C16-C15 & $120.53(19)$ \\
\hline C17-C16-H16 & 119.7 & C15-C16-H16 & 119.7 \\
\hline C14-C15-C16 & 119.42(19) & C14-C15-H15 & 120.3 \\
\hline C16-C15-H15 & 120.3 & O3-C19-H19A & 109.5 \\
\hline O3-C19-H19B & 109.5 & H19A-C19-H19B & 109.5 \\
\hline O3-C19-H19C & 109.5 & H19A-C19-H19C & 109.5 \\
\hline
\end{tabular}


H19B-C19-H19C 109.5

Table S6. Torsion angles $\left(^{\circ}\right)$ for g061.

\begin{tabular}{|c|c|c|c|}
\hline $\mathrm{C} 4-\mathrm{C} 5-\mathrm{C} 6-\mathrm{C} 7$ & $0.0(3)$ & C19-O3-C18-O2 & $-1.0(3)$ \\
\hline C19-O3-C18-C1 & $179.89(17)$ & $\mathrm{C} 6-\mathrm{C} 5-\mathrm{C} 4-\mathrm{C} 9$ & $0.7(3)$ \\
\hline C6-C5-C4-C3 & $-177.24(18)$ & $\mathrm{C} 8-\mathrm{C} 9-\mathrm{C} 4-\mathrm{C} 5$ & $-0.3(3)$ \\
\hline $\mathrm{C} 8-\mathrm{C} 9-\mathrm{C} 4-\mathrm{C} 3$ & $177.50(18)$ & $\mathrm{C} 4-\mathrm{C} 9-\mathrm{C} 8-\mathrm{C} 7$ & $-0.7(3)$ \\
\hline $\mathrm{C} 5-\mathrm{C} 6-\mathrm{C} 7-\mathrm{C} 8$ & $-1.2(3)$ & C5-C6-C7-Cl1 & $177.48(15)$ \\
\hline C9-C8-C7-C6 & $1.5(3)$ & C9-C8-C7-Cl1 & $-177.14(15)$ \\
\hline C12-C11-C10-C1 & 169.69(19) & $\mathrm{C} 5-\mathrm{C} 4-\mathrm{C} 3-\mathrm{C} 2$ & $-176.8(2)$ \\
\hline $\mathrm{C} 9-\mathrm{C} 4-\mathrm{C} 3-\mathrm{C} 2$ & $5.4(3)$ & $\mathrm{C} 4-\mathrm{C} 3-\mathrm{C} 2-\mathrm{C} 1$ & $-174.74(18)$ \\
\hline C15-C14-C13-C12 & $-0.5(3)$ & C14-C13-C12-C17 & $-1.5(3)$ \\
\hline C14-C13-C12-C11 & $174.36(18)$ & $\mathrm{C} 10-\mathrm{C} 11-\mathrm{C} 12-\mathrm{C} 17$ & $9.2(3)$ \\
\hline C10-C11-C12-C13 & $-166.5(2)$ & $\mathrm{C} 13-\mathrm{C} 12-\mathrm{C} 17-\mathrm{C} 16$ & $2.0(3)$ \\
\hline C11-C12-C17-C16 & $-173.68(19)$ & C11-C10-C1-O1 & $17.2(3)$ \\
\hline $\mathrm{C} 11-\mathrm{C} 10-\mathrm{C} 1-\mathrm{C} 2$ & $143.0(2)$ & $\mathrm{C} 11-\mathrm{C} 10-\mathrm{C} 1-\mathrm{C} 18$ & $-100.3(2)$ \\
\hline $\mathrm{C} 3-\mathrm{C} 2-\mathrm{C} 1-\mathrm{O} 1$ & $-15.7(3)$ & $\mathrm{C} 3-\mathrm{C} 2-\mathrm{C} 1-\mathrm{C} 10$ & $-140.6(2)$ \\
\hline C3-C2-C1-C18 & $101.5(2)$ & $\mathrm{O} 2-\mathrm{C} 18-\mathrm{C} 1-\mathrm{O} 1$ & $8.1(3)$ \\
\hline $\mathrm{O} 3-\mathrm{C} 18-\mathrm{C} 1-\mathrm{O} 1$ & $-172.81(16)$ & $\mathrm{O} 2-\mathrm{C} 18-\mathrm{C} 1-\mathrm{C} 10$ & $127.0(2)$ \\
\hline
\end{tabular}




$\begin{array}{llll}\mathrm{O} 3-\mathrm{C} 18-\mathrm{C} 1-\mathrm{C} 10 & -54.0(2) & \mathrm{O} 2-\mathrm{C} 18-\mathrm{C} 1-\mathrm{C} 2 & -111.6(2) \\ \mathrm{O} 3-\mathrm{C} 18-\mathrm{C} 1-\mathrm{C} 2 & 67.5(2) & \mathrm{C} 12-\mathrm{C} 17-\mathrm{C} 16-\mathrm{C} 15 & -0.4(3) \\ \mathrm{C} 13-\mathrm{C} 14-\mathrm{C} 15-\mathrm{C} 16 & 2.1(3) & \mathrm{C} 17-\mathrm{C} 16-\mathrm{C} 15-\mathrm{C} 14 & -1.6(3)\end{array}$

Table S7. Anisotropic atomic displacement parameters $\left(\AA^{2}\right)$ for $\mathrm{g} 061$.

The anisotropic atomic displacement factor exponent takes the form: $-2 \pi^{2}\left[h^{2} a^{* 2} U_{11}+\ldots+2 h k a^{*} b^{*} U_{12}\right]$

$$
\begin{array}{llllll}
\mathbf{U}_{11} & \mathbf{U}_{22} & \mathbf{U}_{33} & \mathbf{U}_{23} & \mathbf{U}_{13} & \mathbf{U}_{12}
\end{array}
$$

\begin{tabular}{|c|c|c|c|c|c|c|}
\hline $\mathrm{Cl1}$ & $0.0195(2)$ & $0.0333(3)$ & $0.0183(2)$ & $-0.0060(2)$ & $-0.00341(17)$ & $0.0015(2)$ \\
\hline $\mathrm{O} 3$ & $0.0242(8)$ & $0.0210(8)$ & $0.0202(8)$ & $-0.0043(6)$ & $0.0050(6)$ & $0.0009(6)$ \\
\hline $\mathrm{O} 1$ & $0.0190(7)$ & $0.0259(8)$ & $0.0211(8)$ & $0.0049(6)$ & $0.0029(6)$ & $-0.0067(6)$ \\
\hline $\mathrm{O} 2$ & $0.0463(10)$ & $0.0198(8)$ & $0.0232(8)$ & $-0.0023(7)$ & $0.0025(7)$ & $-0.0093(7)$ \\
\hline $\mathrm{C} 5$ & $0.0160(9)$ & $0.0159(10)$ & $0.0174(10)$ & $-0.0011(8)$ & $0.0040(8)$ & $-0.0009(7)$ \\
\hline C6 & $0.0215(10)$ & $0.0197(10)$ & $0.0144(10)$ & $0.0008(8)$ & $0.0047(8)$ & $0.0027(8)$ \\
\hline C9 & $0.0192(9)$ & $0.0165(10)$ & $0.0164(10)$ & $0.0020(7)$ & $0.0033(8)$ & $-0.0001(7)$ \\
\hline $\mathrm{C} 18$ & $0.0225(10)$ & $0.0209(10)$ & $0.0127(9)$ & $0.0011(8)$ & $-0.0045(8)$ & $-0.0017(8)$ \\
\hline $\mathrm{C} 4$ & $0.0170(9)$ & $0.0136(10)$ & $0.0151(9)$ & $-0.0017(7)$ & $0.0027(7)$ & $0.0023(7)$ \\
\hline $\mathrm{C} 8$ & $0.0170(9)$ & $0.0160(9)$ & $0.0223(11)$ & $-0.0009(8)$ & $0.0033(8)$ & $-0.0013(7)$ \\
\hline $\mathrm{C} 7$ & $0.0163(8)$ & $0.0170(9)$ & $0.0180(9)$ & $-0.0046(9)$ & $-0.0012(7)$ & $0.0027(8)$ \\
\hline C11 & $0.0171(9)$ & $0.0200(10)$ & $0.0172(10)$ & $-0.0019(8)$ & $0.0039(8)$ & $-0.0036(8)$ \\
\hline $\mathrm{C} 10$ & $0.0191(9)$ & $0.0177(10)$ & $0.0144(10)$ & $-0.0011(8)$ & $0.0033(8)$ & $-0.0028(8)$ \\
\hline C3 & $0.0159(9)$ & $0.0151(9)$ & $0.0189(10)$ & $-0.0017(8)$ & $0.0038(8)$ & $-0.0003(7)$ \\
\hline $\mathrm{C} 2$ & $0.0164(8)$ & $0.0166(8)$ & $0.0163(9)$ & $0.0001(9)$ & $0.0017(7)$ & $-0.0024(8)$ \\
\hline C14 & $0.0169(9)$ & $0.0243(11)$ & $0.0181(11)$ & $-0.0032(9)$ & $0.0015(8)$ & $0.0042(8)$ \\
\hline $\mathrm{C} 13$ & $0.0148(9)$ & $0.0214(10)$ & $0.0190(10)$ & $-0.0025(9)$ & $0.0047(8)$ & $-0.0009(8)$ \\
\hline
\end{tabular}




$$
\begin{array}{llllll}
\mathbf{U}_{11} & \mathbf{U}_{22} & \mathbf{U}_{33} & \mathbf{U}_{23} & \mathbf{U}_{13} & \mathbf{U}_{12}
\end{array}
$$

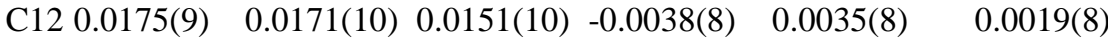
$\begin{array}{llllll}\text { C17 0.0162(9) } & 0.0175(10) & 0.0218(11) & -0.0014(9) & 0.0015(8) & -0.0006(8)\end{array}$
$\begin{array}{lllllll}\mathrm{C} 1 & 0.0154(9) & 0.0219(10) & 0.0165(10) & 0.0011(8) & 0.0024(8) & -0.0039(7)\end{array}$

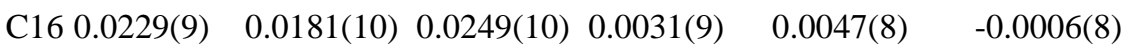
$\begin{array}{llllll}\text { C15 0.0244(10) } & 0.0204(11) & 0.0184(10) & 0.0029(8) & 0.0033(8) & 0.0062(8)\end{array}$
$\begin{array}{llllll}\text { C19 0.0412(13) } & 0.0279(12) & 0.0196(12) & -0.0039(10) & 0.0047(10) & 0.0114(10)\end{array}$

Table S8. Hydrogen atomic coordinates and isotropic atomic displacement parameters $\left(\AA^{2}\right)$ for g061.

\begin{tabular}{|c|c|c|c|c|}
\hline & $\mathbf{x} / \mathbf{a}$ & $\mathbf{y} / \mathbf{b}$ & $\mathbf{z} / \mathbf{c}$ & $\mathbf{U}(\mathbf{e q})$ \\
\hline H1 & 0.9161 & 0.7917 & 0.6974 & 0.033 \\
\hline H5 & 0.5278 & 0.7066 & 0.4513 & 0.02 \\
\hline H6 & 0.2455 & 0.6823 & 0.3496 & 0.022 \\
\hline H9 & 0.1175 & 0.4783 & 0.5855 & 0.021 \\
\hline H8 & -0.1636 & 0.4508 & 0.4841 & 0.022 \\
\hline H11 & 1.1085 & 0.4968 & 0.7880 & 0.022 \\
\hline H10 & 0.6669 & 0.3870 & 0.7897 & 0.02 \\
\hline H3 & 0.6631 & 0.6854 & 0.5809 & 0.02 \\
\hline $\mathrm{H} 2$ & 0.4114 & 0.4967 & 0.6730 & 0.02 \\
\hline H14 & 1.5146 & 0.2234 & 0.9893 & 0.024 \\
\hline H13 & 1.3911 & 0.3985 & 0.8856 & 0.022 \\
\hline $\mathrm{H} 17$ & 0.7731 & 0.1710 & 0.8721 & 0.022 \\
\hline H16 & 0.8958 & -0.0007 & 0.9768 & 0.026 \\
\hline H15 & 1.2640 & 0.0292 & 1.0377 & 0.025 \\
\hline H19A & 0.4730 & 0.8975 & 0.8836 & 0.044 \\
\hline
\end{tabular}




$\begin{array}{rrrrr} & \mathbf{x} / \mathbf{a} & \mathbf{y} / \mathbf{b} & \mathbf{z} / \mathbf{c} & \mathbf{U}(\mathbf{e q}) \\ \text { H19B } & 0.2416 & 0.7880 & 0.8730 & 0.044 \\ \text { H19C } & 0.2865 & 0.9379 & 0.8138 & 0.044\end{array}$




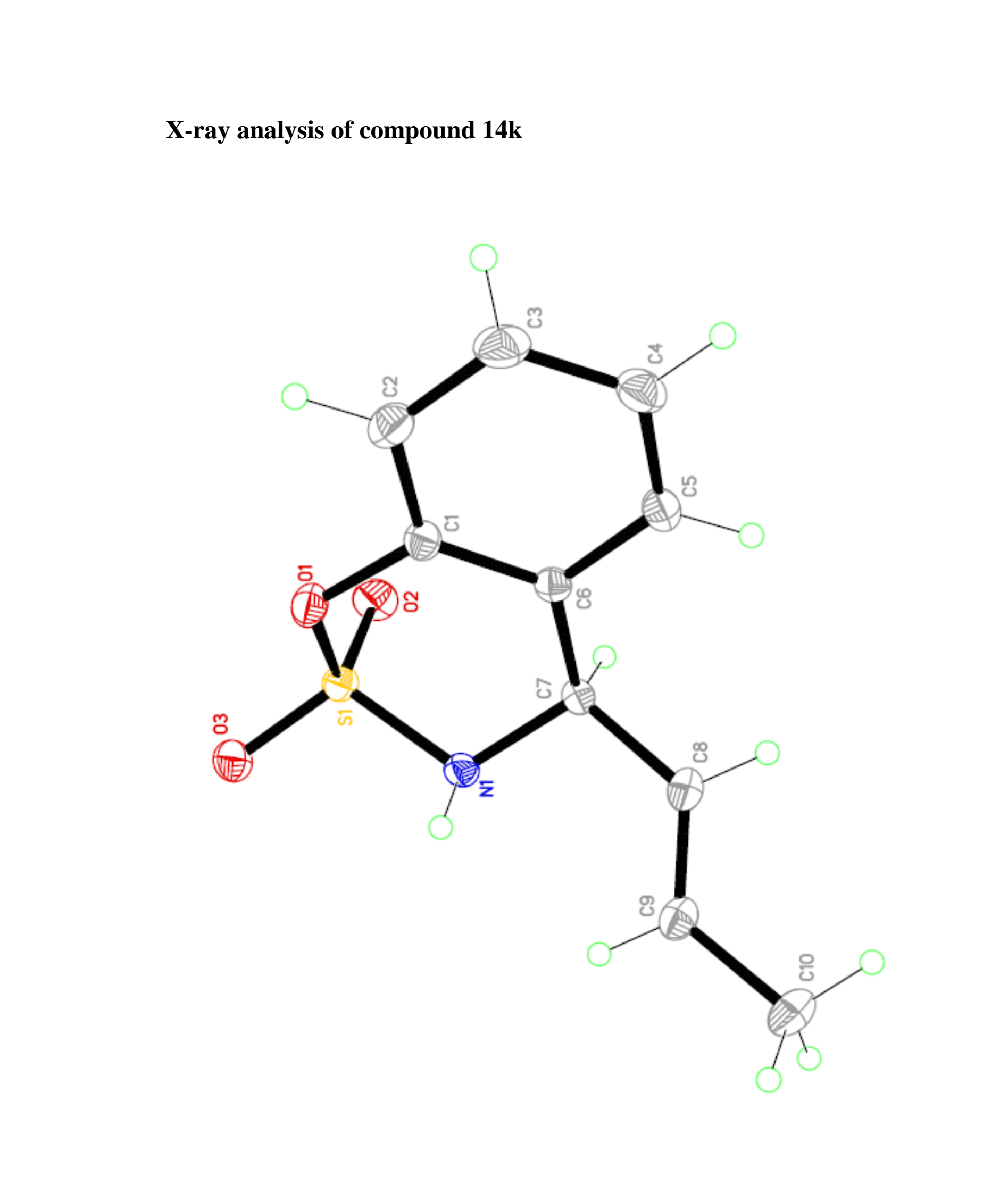


Table 1. Crystal data and structure refinement for G114.

Identification code G114

Empirical formula C10 H11 N O3 S

Formula weight 225.26

Temperature 100(2) K

Wavelength 0.71073 ?

Crystal system Monoclinic

Space group P21

Unit cell dimensions $\mathrm{a}=9.1983(10) ? \quad \mathrm{a}=90$ ?

$$
\begin{array}{ll}
\mathrm{b}=16.7173(18) ? & \mathrm{~b}=90.549(2) ? \\
\mathrm{c}=13.7123(15) ? & \mathrm{~g}=90 ?
\end{array}
$$

Volume 2108.5(4) ?3

Z 8

Density (calculated) $1.419 \mathrm{Mg} / \mathrm{m} 3$

Absorption coefficient $\quad 0.293 \mathrm{~mm}-1$

$\mathrm{F}(000) \quad 944$

Crystal size $\quad 0.580 \times 0.360 \times 0.260 \mathrm{~mm} 3$

Theta range for data collection 1.921 to 28.275 ?

Index ranges $\quad-12<=\mathrm{h}<=12,-22<=\mathrm{k}<=22,-18<=1<=18$

Reflections collected $\quad 24981$

Independent reflections $10429[\mathrm{R}(\mathrm{int})=0.0246]$

Completeness to theta $=25.242 ? \quad 99.9 \%$

Absorption correction Semi-empirical from equivalents

Max. and min. transmission $\quad 0.7457$ and 0.6473

Refinement method Full-matrix least-squares on F2

Data / restraints / parameters 10429/1/561

Goodness-of-fit on F2 1.028

Final R indices $[\mathrm{I}>2 \operatorname{sigma}(\mathrm{I})] \quad \mathrm{R} 1=0.0285, \mathrm{wR} 2=0.0741$

$\mathrm{R}$ indices (all data) $\mathrm{R} 1=0.0292, \mathrm{wR} 2=0.0746$

Absolute structure parameter $0.031(14)$

Extinction coefficient $\quad \mathrm{n} / \mathrm{a}$

Largest diff. peak and hole $\quad 0.398$ and -0.277 e.?-3

Table 2. Atomic coordinates ( $\mathrm{x}$ 104) and equivalent isotropic displacement parameters (?2x 103)

for G114. $\mathrm{U}(\mathrm{eq})$ is defined as one third of the trace of the orthogonalized Uij tensor.

\begin{tabular}{|c|c|c|c|}
\hline$S(1) 10 S$ & 7022 & 642 & 15( \\
\hline 4 & $831^{\circ}$ & 5902 & $15(1$ \\
\hline 3) 2 & 191 & 9 & 17 \\
\hline$(4$ & 308 & 85 & $15(1$ \\
\hline t) & $7515(1)$ & $7375(1)$ & 18( \\
\hline (2) $9603(2)$ & $3(1)$ & $6570(1)$ & 22( \\
\hline
\end{tabular}

$\begin{array}{llll}\mathrm{x} & \mathrm{y} & \mathrm{z} & \mathrm{U}\end{array}$




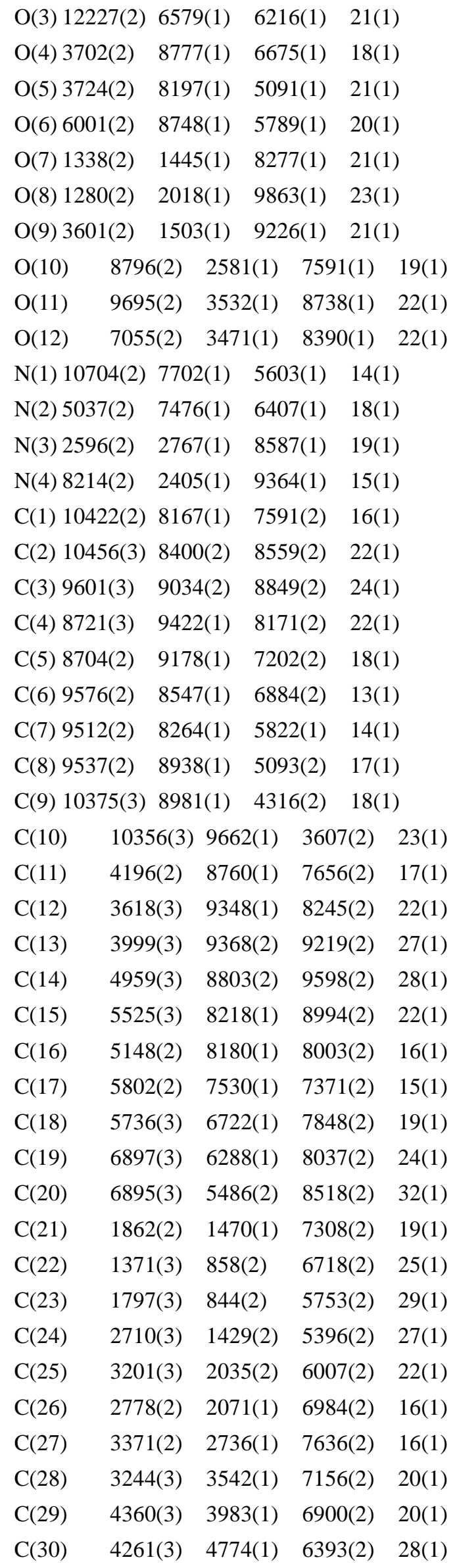




$\begin{array}{lllll}\mathrm{C}(31) & 7838(2) & 1935(1) & 7381(2) & 16(1) \\ \mathrm{C}(32) & 7828(3) & 1695(1) & 6414(2) & 22(1) \\ \mathrm{C}(33) & 6901(3) & 1082(1) & 6129(2) & 23(1) \\ \mathrm{C}(34) & 6008(3) & 724(1) & 6806(2) & 21(1) \\ \mathrm{C}(35) & 6059(2) & 966(1) & 7772(2) & 17(1) \\ \mathrm{C}(36) & 6995(2) & 1575(1) & 8091(1) & 14(1) \\ \mathrm{C}(37) & 7010(2) & 1841(1) & 9157(1) & 14(1) \\ \mathrm{C}(38) & 7071(3) & 1148(1) & 9860(2) & 20(1) \\ \mathrm{C}(39) & 7990(3) & 1050(2) & 10591(2) & 24(1) \\ \mathrm{C}(40) & 7976(3) & 344(2) & 11270(2) & 32(1)\end{array}$

Table 3. Bond lengths [臸 and angles [癩 for G114.

\begin{tabular}{ll}
\hline $\mathrm{S}(1)-\mathrm{O}(2)$ & $1.4179(17)$ \\
$\mathrm{S}(1)-\mathrm{O}(3)$ & $1.4240(17)$ \\
$\mathrm{S}(1)-\mathrm{O}(1)$ & $1.5904(16)$ \\
$\mathrm{S}(1)-\mathrm{N}(1)$ & $1.6088(18)$ \\
$\mathrm{S}(2)-\mathrm{O}(6)$ & $1.4223(16)$ \\
$\mathrm{S}(2)-\mathrm{O}(5)$ & $1.4249(16)$ \\
$\mathrm{S}(2)-\mathrm{O}(4)$ & $1.5943(16)$ \\
$\mathrm{S}(2)-\mathrm{N}(2)$ & $1.600(2)$ \\
$\mathrm{S}(3)-\mathrm{O}(9)$ & $1.4227(16)$ \\
$\mathrm{S}(3)-\mathrm{O}(8)$ & $1.4256(16)$ \\
$\mathrm{S}(3)-\mathrm{O}(7)$ & $1.5915(17)$ \\
$\mathrm{S}(3)-\mathrm{N}(3)$ & $1.603(2)$ \\
$\mathrm{S}(4)-\mathrm{O}(12)$ & $1.4218(16)$ \\
$\mathrm{S}(4)-\mathrm{O}(11)$ & $1.4251(17)$ \\
$\mathrm{S}(4)-\mathrm{O}(10)$ & $1.5915(16)$ \\
$\mathrm{S}(4)-\mathrm{N}(4)$ & $1.6059(18)$ \\
$\mathrm{O}(1)-\mathrm{C}(1)$ & $1.419(3)$ \\
$\mathrm{O}(4)-\mathrm{C}(11)$ & $1.416(3)$ \\
$\mathrm{O}(7)-\mathrm{C}(21)$ & $1.418(3)$ \\
$\mathrm{O}(10)-\mathrm{C}(31)$ & $1.422(3)$ \\
$\mathrm{N}(1)-\mathrm{C}(7)$ & $1.478(3)$ \\
$\mathrm{N}(1)-\mathrm{H}(1 \mathrm{~N})$ & $0.82(3)$ \\
$\mathrm{N}(2)-\mathrm{C}(17)$ & $1.494(3)$ \\
$\mathrm{N}(2)-\mathrm{H}(2 \mathrm{~N})$ & $0.94(3)$ \\
$\mathrm{N}(3)-\mathrm{C}(27)$ & $1.493(3)$ \\
$\mathrm{N}(3)-\mathrm{H}(3 \mathrm{~N})$ & $0.84(4)$ \\
$\mathrm{N}(4)-\mathrm{C}(37)$ & $1.479(3)$ \\
$\mathrm{N}(4)-\mathrm{H}(4 \mathrm{~N})$ & $0.86(3)$ \\
$\mathrm{C}(1)-\mathrm{C}(2)$ & $1.383(3)$ \\
$\mathrm{C}(1)-\mathrm{C}(6)$ & $1.390(3)$ \\
$\mathrm{C}(2)-\mathrm{C}(3)$ & $1.382(3)$ \\
&
\end{tabular}




$\begin{array}{ll}\mathrm{C}(2)-\mathrm{H}(2) & 0.9500 \\ \mathrm{C}(3)-\mathrm{C}(4) & 1.387(3) \\ \mathrm{C}(3)-\mathrm{H}(3) & 0.9500 \\ \mathrm{C}(4)-\mathrm{C}(5) & 1.389(3) \\ \mathrm{C}(4)-\mathrm{H}(4) & 0.9500 \\ \mathrm{C}(5)-\mathrm{C}(6) & 1.398(3) \\ \mathrm{C}(5)-\mathrm{H}(5) & 0.9500 \\ \mathrm{C}(6)-\mathrm{C}(7) & 1.531(3) \\ \mathrm{C}(7)-\mathrm{C}(8) & 1.507(3) \\ \mathrm{C}(7)-\mathrm{H}(7) & 1.0000 \\ \mathrm{C}(8)-\mathrm{C}(9) & 1.322(3) \\ \mathrm{C}(8)-\mathrm{H}(8) & 0.9500 \\ \mathrm{C}(9)-\mathrm{C}(10) & 1.496(3) \\ \mathrm{C}(9)-\mathrm{H}(9) & 0.9500 \\ \mathrm{C}(10)-\mathrm{H}(10 \mathrm{~A}) & 0.9800 \\ \mathrm{C}(10)-\mathrm{H}(10 \mathrm{~B}) & 0.9800 \\ \mathrm{C}(10)-\mathrm{H}(10 \mathrm{C}) & 0.9800 \\ \mathrm{C}(11)-\mathrm{C}(12) & 1.382(3) \\ \mathrm{C}(11)-\mathrm{C}(16) & 1.387(3) \\ \mathrm{C}(12)-\mathrm{C}(13) & 1.377(4) \\ \mathrm{C}(12)-\mathrm{H}(12) & 0.9500 \\ \mathrm{C}(13)-\mathrm{C}(14) & 1.392(4) \\ \mathrm{C}(13)-\mathrm{H}(13) & 0.9500 \\ \mathrm{C}(14)-\mathrm{C}(15) & 1.386(3) \\ \mathrm{C}(14)-\mathrm{H}(14) & 0.9500 \\ \mathrm{C}(15)-\mathrm{C}(16) & 1.400(3) \\ \mathrm{C}(15)-\mathrm{H}(15) & 0.9500 \\ \mathrm{C}(16)-\mathrm{C}(17) & 1.519(3) \\ \mathrm{C}(17)-\mathrm{C}(18) & 1.502(3) \\ \mathrm{C}(17)-\mathrm{H}(17) & 1.0000 \\ \mathrm{C}(18)-\mathrm{C}(19) & 1.315(3) \\ \mathrm{C}(18)-\mathrm{H}(18) & 0.9500 \\ \mathrm{C}(19)-\mathrm{C}(20) & 1.494(3) \\ \mathrm{C}(19)-\mathrm{H}(19) & 0.9500 \\ \mathrm{C}(20)-\mathrm{H}(20 \mathrm{~A}) & 0.9800 \\ \mathrm{C}(20)-\mathrm{H}(20 \mathrm{~B}) & 0.9800 \\ \mathrm{C}(20)-\mathrm{H}(20 \mathrm{C}) & 0.9800 \\ \mathrm{C}(21)-\mathrm{C}(22) & 1.378(3) \\ \mathrm{C}(21)-\mathrm{C}(26) & 1.386(3) \\ \mathrm{C}(22)-\mathrm{C}(23) & 1.385(4) \\ \mathrm{C}(22)-\mathrm{H}(22) & 0.9500 \\ \mathrm{C}(23)-\mathrm{C}(24) & 1.382(4) \\ \mathrm{C}(23)-\mathrm{H}(23) & 0.9500 \\ \mathrm{C}(24)-\mathrm{C}(25) & 1.388(3) \\ & \end{array}$




$\begin{array}{ll}\mathrm{C}(24)-\mathrm{H}(24) & 0.9500 \\ \mathrm{C}(25)-\mathrm{C}(26) & 1.400(3) \\ \mathrm{C}(25)-\mathrm{H}(25) & 0.9500 \\ \mathrm{C}(26)-\mathrm{C}(27) & 1.525(3) \\ \mathrm{C}(27)-\mathrm{C}(28) & 1.503(3) \\ \mathrm{C}(27)-\mathrm{H}(27) & 1.0000 \\ \mathrm{C}(28)-\mathrm{C}(29) & 1.314(3) \\ \mathrm{C}(28)-\mathrm{H}(28) & 0.9500 \\ \mathrm{C}(29)-\mathrm{C}(30) & 1.496(3) \\ \mathrm{C}(29)-\mathrm{H}(29) & 0.9500 \\ \mathrm{C}(30)-\mathrm{H}(30 \mathrm{~A}) & 0.9800 \\ \mathrm{C}(30)-\mathrm{H}(30 \mathrm{~B}) & 0.9800 \\ \mathrm{C}(30)-\mathrm{H}(30 \mathrm{C}) & 0.9800 \\ \mathrm{C}(31)-\mathrm{C}(32) & 1.385(3) \\ \mathrm{C}(31)-\mathrm{C}(36) & 1.388(3) \\ \mathrm{C}(32)-\mathrm{C}(33) & 1.387(3) \\ \mathrm{C}(32)-\mathrm{H}(32) & 0.9500 \\ \mathrm{C}(33)-\mathrm{C}(34) & 1.382(3) \\ \mathrm{C}(33)-\mathrm{H}(33) & 0.9500 \\ \mathrm{C}(34)-\mathrm{C}(35) & 1.386(3) \\ \mathrm{C}(34)-\mathrm{H}(34) & 0.9500 \\ \mathrm{C}(35)-\mathrm{C}(36) & 1.401(3) \\ \mathrm{C}(35)-\mathrm{H}(35) & 0.9500 \\ \mathrm{C}(36)-\mathrm{C}(37) & 1.527(3) \\ \mathrm{C}(37)-\mathrm{C}(38) & 1.508(3) \\ \mathrm{C}(37)-\mathrm{H}(37) & 1.0000 \\ \mathrm{C}(38)-\mathrm{C}(39) & 1.315(3) \\ \mathrm{C}(38)-\mathrm{H}(38) & 0.9500 \\ \mathrm{C}(39)-\mathrm{C}(40) & 1.504(3) \\ \mathrm{C}(39)-\mathrm{H}(39) & 0.9500 \\ \mathrm{C}(40)-\mathrm{H}(40 \mathrm{~A}) & 0.9800 \\ \mathrm{C}(40)-\mathrm{H}(40 \mathrm{~B}) & 0.9800 \\ \mathrm{C}(40)-\mathrm{H}(40 \mathrm{C}) & 0.9800 \\ \mathrm{O}(2)-\mathrm{S}(1)-\mathrm{O}(3) & 120.54(10) \\ \mathrm{O}(2)-\mathrm{S}(1)-\mathrm{O}(1) & 108.88(10) \\ \mathrm{O}(3)-\mathrm{S}(1)-\mathrm{O}(1) & 103.91(9) \\ \mathrm{O}(2)-\mathrm{S}(1)-\mathrm{N}(1) & 108.73(10) \\ \mathrm{O}(3)-\mathrm{S}(1)-\mathrm{N}(1) & 109.75(10) \\ \mathrm{O}(1)-\mathrm{S}(1)-\mathrm{N}(1) & 103.66(9) \\ \mathrm{O}(6)-\mathrm{S}(2)-\mathrm{O}(5) & 120.54(10) \\ \mathrm{O}(5)-\mathrm{S}(2)-\mathrm{O}(4) & 108.42(10) \\ \mathrm{O}(6)-\mathrm{S}(2)-\mathrm{N}(2) & 108.83(9) \\ & \end{array}$




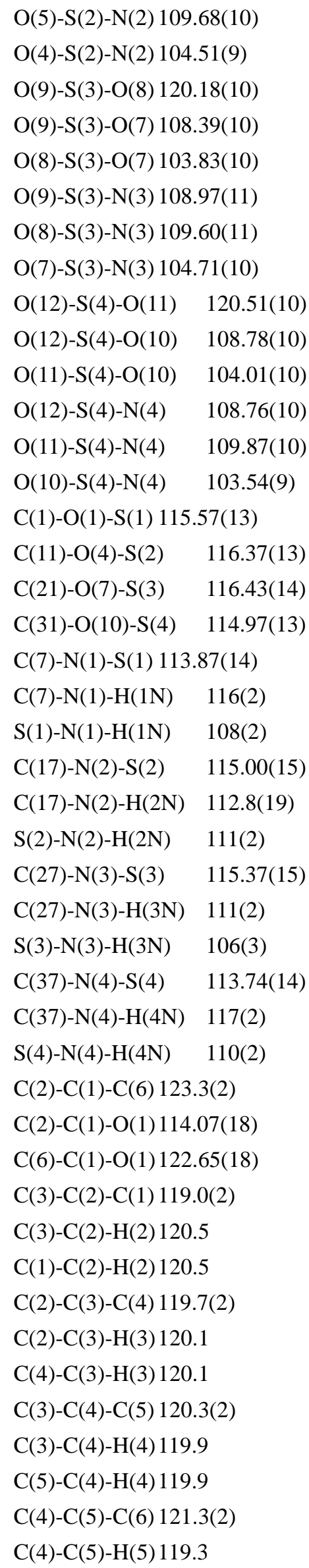


$\mathrm{C}(6)-\mathrm{C}(5)-\mathrm{H}(5) 119.3$

$\mathrm{C}(1)-\mathrm{C}(6)-\mathrm{C}(5)$ 116.43(19)

$\mathrm{C}(1)-\mathrm{C}(6)-\mathrm{C}(7)$ 122.59(18)

$\mathrm{C}(5)-\mathrm{C}(6)-\mathrm{C}(7)$ 120.86(18)

$\mathrm{N}(1)-\mathrm{C}(7)-\mathrm{C}(8)$ 108.94(16)

$\mathrm{N}(1)-\mathrm{C}(7)-\mathrm{C}(6) 111.53(16)$

$\mathrm{C}(8)-\mathrm{C}(7)-\mathrm{C}(6)$ 113.55(17)

$\mathrm{N}(1)-\mathrm{C}(7)-\mathrm{H}(7) 107.5$

$\mathrm{C}(8)-\mathrm{C}(7)-\mathrm{H}(7) 107.5$

$\mathrm{C}(6)-\mathrm{C}(7)-\mathrm{H}(7) 107.5$

$\mathrm{C}(9)-\mathrm{C}(8)-\mathrm{C}(7) 126.0(2)$

$\mathrm{C}(9)-\mathrm{C}(8)-\mathrm{H}(8) 117.0$

$\mathrm{C}(7)-\mathrm{C}(8)-\mathrm{H}(8) 117.0$

$\mathrm{C}(8)-\mathrm{C}(9)-\mathrm{C}(10) \quad 124.2(2)$

C(8)-C(9)-H(9) 117.9

$\mathrm{C}(10)-\mathrm{C}(9)-\mathrm{H}(9) \quad 117.9$

C(9)-C(10)-H(10A) 109.5

C(9)-C(10)-H(10B) 109.5

$\mathrm{H}(10 \mathrm{~A})-\mathrm{C}(10)-\mathrm{H}(10 \mathrm{~B}) \quad 109.5$

$\mathrm{C}(9)-\mathrm{C}(10)-\mathrm{H}(10 \mathrm{C}) \quad 109.5$

$\mathrm{H}(10 \mathrm{~A})-\mathrm{C}(10)-\mathrm{H}(10 \mathrm{C}) \quad 109.5$

$\mathrm{H}(10 \mathrm{~B})-\mathrm{C}(10)-\mathrm{H}(10 \mathrm{C}) \quad 109.5$

$\mathrm{C}(12)-\mathrm{C}(11)-\mathrm{C}(16) \quad 122.8(2)$

$\mathrm{C}(12)-\mathrm{C}(11)-\mathrm{O}(4) \quad 114.8(2)$

$\mathrm{C}(16)-\mathrm{C}(11)-\mathrm{O}(4) \quad 122.42(18)$

C(13)-C(12)-C(11) 119.3(2)

$\mathrm{C}(13)-\mathrm{C}(12)-\mathrm{H}(12) \quad 120.4$

$\mathrm{C}(11)-\mathrm{C}(12)-\mathrm{H}(12) \quad 120.4$

$\mathrm{C}(12)-\mathrm{C}(13)-\mathrm{C}(14) \quad 120.0(2)$

$\mathrm{C}(12)-\mathrm{C}(13)-\mathrm{H}(13) \quad 120.0$

$\mathrm{C}(14)-\mathrm{C}(13)-\mathrm{H}(13) \quad 120.0$

$\mathrm{C}(15)-\mathrm{C}(14)-\mathrm{C}(13) \quad 119.8(2)$

$\mathrm{C}(15)-\mathrm{C}(14)-\mathrm{H}(14) \quad 120.1$

$\mathrm{C}(13)-\mathrm{C}(14)-\mathrm{H}(14) \quad 120.1$

C(14)-C(15)-C(16) 121.4(2)

$\mathrm{C}(14)-\mathrm{C}(15)-\mathrm{H}(15) \quad 119.3$

$\mathrm{C}(16)-\mathrm{C}(15)-\mathrm{H}(15) \quad 119.3$

$\mathrm{C}(11)-\mathrm{C}(16)-\mathrm{C}(15) \quad 116.8(2)$

$\mathrm{C}(11)-\mathrm{C}(16)-\mathrm{C}(17) \quad 123.75(18)$

$\mathrm{C}(15)-\mathrm{C}(16)-\mathrm{C}(17) \quad 119.40(19)$

$\mathrm{N}(2)-\mathrm{C}(17)-\mathrm{C}(18) \quad$ 108.09(18)

$\mathrm{N}(2)-\mathrm{C}(17)-\mathrm{C}(16) \quad 111.31(17)$

$\mathrm{C}(18)-\mathrm{C}(17)-\mathrm{C}(16) \quad 112.15(17)$

$\mathrm{N}(2)-\mathrm{C}(17)-\mathrm{H}(17) \quad 108.4$ 


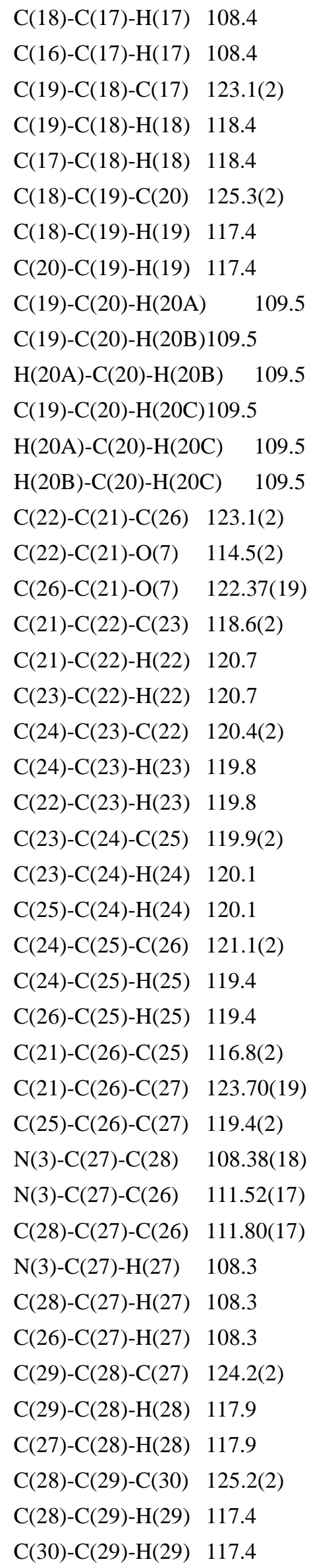




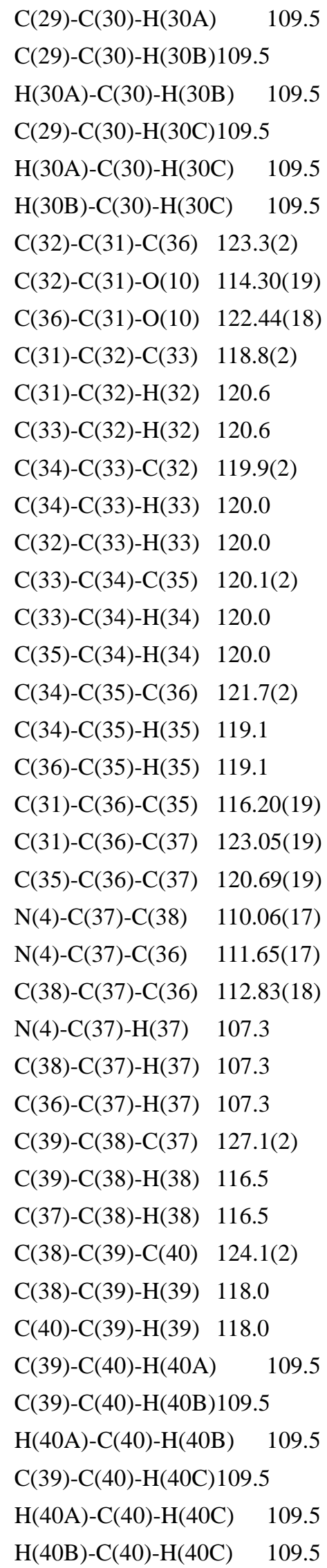

Symmetry transformations used to generate equivalent atoms: 
Table 4. Anisotropic displacement parameters (?2x 103) for G114. The anisotropic displacement factor exponent takes the form: $\quad-2 \mathrm{p} 2\left[\mathrm{~h} 2 \mathrm{a} * 2 \mathrm{U} 11+\ldots+2 \mathrm{~h} \mathrm{k} \mathrm{a}^{*} \mathrm{~b} * \mathrm{U} 12\right.$ ]

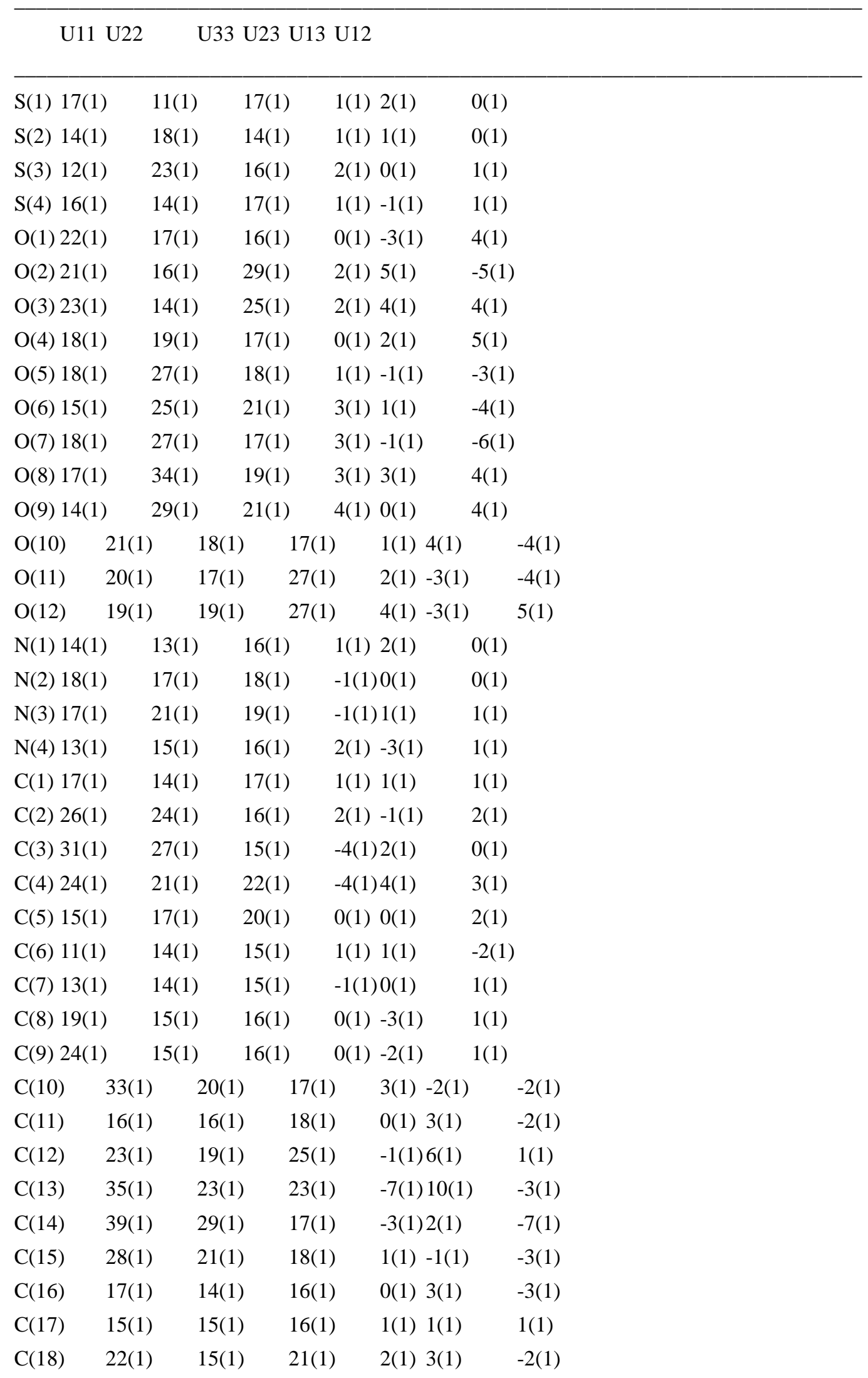




$\begin{array}{llllll}\mathrm{C}(19) & 31(1) & 21(1) & 20(1) & 5(1) 8(1) & 6(1) \\ \mathrm{C}(20) & 49(2) & 20(1) & 26(1) & 6(1) 7(1) & 11(1) \\ \mathrm{C}(21) & 18(1) & 23(1) & 15(1) & 2(1)-2(1) & -2(1) \\ \mathrm{C}(22) & 26(1) & 24(1) & 25(1) & 2(1)-6(1) & -8(1) \\ \mathrm{C}(23) & 37(1) & 27(1) & 23(1) & -5(1)-9(1) & -6(1) \\ \mathrm{C}(24) & 34(1) & 31(1) & 17(1) & -1(1)-2(1) & -1(1) \\ \mathrm{C}(25) & 23(1) & 23(1) & 20(1) & 4(1) 0(1) & -2(1) \\ \mathrm{C}(26) & 16(1) & 16(1) & 18(1) & 1(1)-4(1) & 0(1) \\ \mathrm{C}(27) & 14(1) & 16(1) & 16(1) & 1(1)-1(1) & -1(1) \\ \mathrm{C}(28) & 20(1) & 18(1) & 22(1) & 1(1)-2(1) & 4(1) \\ \mathrm{C}(29) & 26(1) & 18(1) & 17(1) & -1(1)-4(1) & 0(1) \\ \mathrm{C}(30) & 43(2) & 18(1) & 22(1) & 2(1)-3(1) & -6(1) \\ \mathrm{C}(31) & 17(1) & 14(1) & 17(1) & 1(1) 0(1) & 0(1) \\ \mathrm{C}(32) & 28(1) & 21(1) & 17(1) & 2(1) 4(1) & 3(1) \\ \mathrm{C}(33) & 33(1) & 20(1) & 16(1) & -3(1)-3(1) & 4(1) \\ \mathrm{C}(34) & 24(1) & 17(1) & 22(1) & -2(1)-4(1) & 2(1) \\ \mathrm{C}(35) & 17(1) & 16(1) & 19(1) & 1(1) 1(1) & 1(1) \\ \mathrm{C}(36) & 14(1) & 14(1) & 13(1) & 1(1)-1(1) & 4(1) \\ \mathrm{C}(37) & 14(1) & 16(1) & 13(1) & 0(1) 1(1) & 0(1) \\ \mathrm{C}(38) & 24(1) & 19(1) & 18(1) & 3(1) 2(1) & -2(1) \\ \mathrm{C}(39) & 27(1) & 22(1) & 22(1) & 4(1) 1(1) & 0(1) \\ \mathrm{C}(40) & 43(2) & 30(1) & 23(1) & 9(1) 1(1) & 3(1)\end{array}$

Table 5. Hydrogen coordinates ( x 104) and isotropic displacement parameters (?2x 103$)$ for G114.

$\begin{array}{llll}\mathrm{x} & \mathrm{y} & \mathrm{z} & \mathrm{U}(\mathrm{eq})\end{array}$

$\mathrm{H}(1 \mathrm{~N}) \quad 11490(30) 7907(18) \quad 5490(20) \quad 20(7)$

$\mathrm{H}(2 \mathrm{~N}) \quad 4210(30) \quad 7149(19) \quad 6420(20) \quad 29(8)$

$\mathrm{H}(3 \mathrm{~N}) \quad 1790(40) \quad 3000(20) \quad 8530(30) \quad 45(10)$

$\mathrm{H}(4 \mathrm{~N}) \quad 9040(40) \quad 2200(20) \quad 9520(20) \quad 30(8)$

$\mathrm{H}(2) 11058 \quad 8127901726$

H(3) 96159205951029

$\mathrm{H}(4) 81289857836927$

$\mathrm{H}(5) 80879444674821$

$\mathrm{H}(7) 85757969572717$

$\mathrm{H}(8) 88929372520120$

$\mathrm{H}(9) 11031 \quad 8553420122$

$\mathrm{H}(10 \mathrm{~A}) \quad 962910056 \quad 380735$

$\mathrm{H}(10 \mathrm{~B}) \quad 10110 \quad 9460295535$

H(10C) $11317 \quad 9914359535$

H(12) 29669734798227 


\begin{tabular}{|c|c|c|}
\hline $\mathrm{H}(13)$ & 36069769963132 & \\
\hline $\mathrm{H}(14)$ & 5226881710269 & 34 \\
\hline $\mathrm{H}(15)$ & 61827834925827 & \\
\hline $\mathrm{H}(17)$ & 68447665725318 & \\
\hline $\mathrm{H}(18)$ & 48136515802123 & \\
\hline $\mathrm{H}(19)$ & 78126502785328 & \\
\hline $\mathrm{H}(20 \mathrm{~A})$ & 58975342869147 & \\
\hline $\mathrm{H}(20 \mathrm{~B})$ & 75005505911047 & \\
\hline $\mathrm{H}(20 \mathrm{C})$ & 72855086806947 & \\
\hline $\mathrm{H}(22)$ & $\begin{array}{lll}753 & 454 & 696930\end{array}$ & \\
\hline $\mathrm{H}(23)$ & 1459431533335 & \\
\hline $\mathrm{H}(24)$ & 30031416473333 & \\
\hline $\mathrm{H}(25)$ & 38342434575826 & \\
\hline $\mathrm{H}(27)$ & 44232627777019 & \\
\hline $\mathrm{H}(28)$ & 22973746703124 & \\
\hline $\mathrm{H}(29)$ & 53033784704924 & \\
\hline $\mathrm{H}(30 \mathrm{~A})$ & 32374912628341 & \\
\hline $\mathrm{H}(30 \mathrm{~B})$ & 47584742576541 & \\
\hline $\mathrm{H}(30 \mathrm{C})$ & 47255186679841 & \\
\hline $\mathrm{H}(32)$ & 84451947595426 & \\
\hline $\mathrm{H}(33)$ & 6881909547027 & \\
\hline $\mathrm{H}(34)$ & 5358312660925 & \\
\hline $\mathrm{H}(35)$ & 5443712823021 & \\
\hline $\mathrm{H}(37)$ & 60792133927517 & \\
\hline $\mathrm{H}(38)$ & 6370738976525 & \\
\hline $\mathrm{H}(39)$ & 8708145110697 & 9 \\
\hline $\mathrm{H}(40 \mathrm{~A})$ & 71384 & 48 \\
\hline $\mathrm{H}(40 \mathrm{~B})$ & 790853211944 & 48 \\
\hline $\mathrm{H}(40 \mathrm{C})$ & $887436 \quad 11191$ & \\
\hline
\end{tabular}

Table 6. Torsion angles [癩 for G114.

\begin{tabular}{|c|c|}
\hline 2) & \\
\hline$(3)-\mathrm{S}(1)-\mathrm{O}(1)-\mathrm{C}(1) 1 \mathrm{C}$ & $9(15)$ \\
\hline $\mathrm{N}(1) \mathrm{S}(1) \mathrm{O}(1) \mathrm{C}(1) 487$ & 6) \\
\hline $\mathrm{O}(6)-\mathrm{S}(2)-\mathrm{O}(4)-\mathrm{C}(11)$ & $-69.75(16)$ \\
\hline $\mathrm{O}(5)-\mathrm{S}(2)-\mathrm{O}(4)-\mathrm{C}(11)$ & $160.93(15)$ \\
\hline 2)-O(4)-C(11) & $45.97(17)$ \\
\hline $\mathrm{O}(9)-\mathrm{S}(3)-\mathrm{O}(7)-\mathrm{C}(21)$ & $-69.92(17)$ \\
\hline $\mathrm{O}(8)-\mathrm{S}(3)-\mathrm{O}(7)-\mathrm{C}(21)$ & $161.23(16)$ \\
\hline $\mathrm{N}(3$ & $46.28(18)$ \\
\hline $\mathrm{O}(12)-\mathrm{S}(4)-\mathrm{O}(10)-\mathrm{C}(31)$ & $-65.43(16)$ \\
\hline $\mathrm{O}(11)-\mathrm{S}(4)-\mathrm{O}(10)-\mathrm{C}(31)$ & $164.97(14)$ \\
\hline $\mathrm{N}(4)-\mathrm{S}(4)-\mathrm{O}(10)-\mathrm{C}(31)$ & $50.12(16)$ \\
\hline
\end{tabular}


$\mathrm{O}(2)-\mathrm{S}(1)-\mathrm{N}(1)-\mathrm{C}(7) 54.07(17)$

$\mathrm{O}(3)-\mathrm{S}(1)-\mathrm{N}(1)-\mathrm{C}(7)-172.15(14)$

$\mathrm{O}(1)-\mathrm{S}(1)-\mathrm{N}(1)-\mathrm{C}(7)-61.65(16)$

$\mathrm{O}(6)-\mathrm{S}(2)-\mathrm{N}(2)-\mathrm{C}(17) \quad$ 57.17(17)

$\mathrm{O}(5)-\mathrm{S}(2)-\mathrm{N}(2)-\mathrm{C}(17) \quad-169.19(14)$

$\mathrm{O}(4)-\mathrm{S}(2)-\mathrm{N}(2)-\mathrm{C}(17) \quad-58.41(17)$

$\mathrm{O}(9)-\mathrm{S}(3)-\mathrm{N}(3)-\mathrm{C}(27) \quad 58.36(18)$

$\mathrm{O}(8)-\mathrm{S}(3)-\mathrm{N}(3)-\mathrm{C}(27) \quad-168.28(15)$

$\mathrm{O}(7)-\mathrm{S}(3)-\mathrm{N}(3)-\mathrm{C}(27) \quad-57.44(17)$

$\mathrm{O}(12)-\mathrm{S}(4)-\mathrm{N}(4)-\mathrm{C}(37) \quad 53.24(17)$

$\mathrm{O}(11)-\mathrm{S}(4)-\mathrm{N}(4)-\mathrm{C}(37) \quad-172.91(14)$

$\mathrm{O}(10)-\mathrm{S}(4)-\mathrm{N}(4)-\mathrm{C}(37) \quad-62.32(16)$

$\mathrm{S}(1)-\mathrm{O}(1)-\mathrm{C}(1)-\mathrm{C}(2)$ 157.93(17)

$\mathrm{S}(1)-\mathrm{O}(1)-\mathrm{C}(1)-\mathrm{C}(6)-23.3(3)$

$\mathrm{C}(6)-\mathrm{C}(1)-\mathrm{C}(2)-\mathrm{C}(3) 0.7(4)$

$\mathrm{O}(1)-\mathrm{C}(1)-\mathrm{C}(2)-\mathrm{C}(3)$ 179.4(2)

$\mathrm{C}(1)-\mathrm{C}(2)-\mathrm{C}(3)-\mathrm{C}(4) 0.4(4)$

$\mathrm{C}(2)-\mathrm{C}(3)-\mathrm{C}(4)-\mathrm{C}(5)-0.3(4)$

$\mathrm{C}(3)-\mathrm{C}(4)-\mathrm{C}(5)-\mathrm{C}(6)-0.8(4)$

$\mathrm{C}(2)-\mathrm{C}(1)-\mathrm{C}(6)-\mathrm{C}(5)-1.8(3)$

$\mathrm{O}(1)-\mathrm{C}(1)-\mathrm{C}(6)-\mathrm{C}(5) 179.62(19)$

$\mathrm{C}(2)-\mathrm{C}(1)-\mathrm{C}(6)-\mathrm{C}(7)-177.9(2)$

$\mathrm{O}(1)-\mathrm{C}(1)-\mathrm{C}(6)-\mathrm{C}(7) 3.5(3)$

$\mathrm{C}(4)-\mathrm{C}(5)-\mathrm{C}(6)-\mathrm{C}(1) 1.8(3)$

$\mathrm{C}(4)-\mathrm{C}(5)-\mathrm{C}(6)-\mathrm{C}(7) 178.0(2)$

$\mathrm{S}(1)-\mathrm{N}(1)-\mathrm{C}(7)-\mathrm{C}(8)$ 171.66(14)

$\mathrm{S}(1)-\mathrm{N}(1)-\mathrm{C}(7)-\mathrm{C}(6)$ 45.5(2)

$\mathrm{C}(1)-\mathrm{C}(6)-\mathrm{C}(7)-\mathrm{N}(1)-14.7(3)$

$\mathrm{C}(5)-\mathrm{C}(6)-\mathrm{C}(7)-\mathrm{N}(1) 169.31(19)$

$\mathrm{C}(1)-\mathrm{C}(6)-\mathrm{C}(7)-\mathrm{C}(8)-138.3(2)$

$\mathrm{C}(5)-\mathrm{C}(6)-\mathrm{C}(7)-\mathrm{C}(8) 45.8(3)$

$\mathrm{N}(1)-\mathrm{C}(7)-\mathrm{C}(8)-\mathrm{C}(9) 7.1(3)$

$\mathrm{C}(6)-\mathrm{C}(7)-\mathrm{C}(8)-\mathrm{C}(9) 132.0(2)$

$\mathrm{C}(7)-\mathrm{C}(8)-\mathrm{C}(9)-\mathrm{C}(10) \quad 179.6(2)$

$\mathrm{S}(2)-\mathrm{O}(4)-\mathrm{C}(11)-\mathrm{C}(12) \quad$ 160.77(16)

$\mathrm{S}(2)-\mathrm{O}(4)-\mathrm{C}(11)-\mathrm{C}(16) \quad-21.3(3)$

$\mathrm{C}(16)-\mathrm{C}(11)-\mathrm{C}(12)-\mathrm{C}(13)$ 0.3(4)

$\mathrm{O}(4)-\mathrm{C}(11)-\mathrm{C}(12)-\mathrm{C}(13) \quad$ 178.2(2)

$\mathrm{C}(11)-\mathrm{C}(12)-\mathrm{C}(13)-\mathrm{C}(14)$ 0.2(4)

$\mathrm{C}(12)-\mathrm{C}(13)-\mathrm{C}(14)-\mathrm{C}(15)-0.3(4)$

$\mathrm{C}(13)-\mathrm{C}(14)-\mathrm{C}(15)-\mathrm{C}(16)-0.2(4)$

$\mathrm{C}(12)-\mathrm{C}(11)-\mathrm{C}(16)-\mathrm{C}(15)-0.7(3)$

$\mathrm{O}(4)-\mathrm{C}(11)-\mathrm{C}(16)-\mathrm{C}(15)-178.42(19)$

$\mathrm{C}(12)-\mathrm{C}(11)-\mathrm{C}(16)-\mathrm{C}(17)-179.8(2)$ 
$\mathrm{O}(4)-\mathrm{C}(11)-\mathrm{C}(16)-\mathrm{C}(17) \quad 2.4(3)$

$\mathrm{C}(14)-\mathrm{C}(15)-\mathrm{C}(16)-\mathrm{C}(11)$ 0.6(3)

C(14)-C(15)-C(16)-C(17) 179.8(2)

$\mathrm{S}(2)-\mathrm{N}(2)-\mathrm{C}(17)-\mathrm{C}(18) \quad 166.73(15)$

$\mathrm{S}(2)-\mathrm{N}(2)-\mathrm{C}(17)-\mathrm{C}(16) \quad 43.1(2)$

$\mathrm{C}(11)-\mathrm{C}(16)-\mathrm{C}(17)-\mathrm{N}(2) \quad-13.3(3)$

$\mathrm{C}(15)-\mathrm{C}(16)-\mathrm{C}(17)-\mathrm{N}(2) \quad 167.58(19)$

$\mathrm{C}(11)-\mathrm{C}(16)-\mathrm{C}(17)-\mathrm{C}(18)-134.5(2)$

C(15)-C(16)-C(17)-C(18) 46.3(3)

$\mathrm{N}(2)-\mathrm{C}(17)-\mathrm{C}(18)-\mathrm{C}(19) \quad 115.8(2)$

C(16)-C(17)-C(18)-C(19) -121.2(2)

$\mathrm{C}(17)-\mathrm{C}(18)-\mathrm{C}(19)-\mathrm{C}(20)$ 179.4(2)

$\mathrm{S}(3)-\mathrm{O}(7)-\mathrm{C}(21)-\mathrm{C}(22) \quad$ 158.46(17)

$\mathrm{S}(3)-\mathrm{O}(7)-\mathrm{C}(21)-\mathrm{C}(26) \quad-22.4(3)$

$\mathrm{C}(26)-\mathrm{C}(21)-\mathrm{C}(22)-\mathrm{C}(23)-0.7(4)$

$\mathrm{O}(7)-\mathrm{C}(21)-\mathrm{C}(22)-\mathrm{C}(23) \quad 178.4(2)$

$\mathrm{C}(21)-\mathrm{C}(22)-\mathrm{C}(23)-\mathrm{C}(24) 0.8(4)$

$\mathrm{C}(22)-\mathrm{C}(23)-\mathrm{C}(24)-\mathrm{C}(25)-0.3(4)$

$\mathrm{C}(23)-\mathrm{C}(24)-\mathrm{C}(25)-\mathrm{C}(26)-0.4(4)$

$\mathrm{C}(22)-\mathrm{C}(21)-\mathrm{C}(26)-\mathrm{C}(25)$ 0.1(3)

$\mathrm{O}(7)-\mathrm{C}(21)-\mathrm{C}(26)-\mathrm{C}(25)-179.0(2)$

$\mathrm{C}(22)-\mathrm{C}(21)-\mathrm{C}(26)-\mathrm{C}(27)-177.9(2)$

$\mathrm{O}(7)-\mathrm{C}(21)-\mathrm{C}(26)-\mathrm{C}(27) \quad 3.1(3)$

$\mathrm{C}(24)-\mathrm{C}(25)-\mathrm{C}(26)-\mathrm{C}(21) 0.5(3)$

$\mathrm{C}(24)-\mathrm{C}(25)-\mathrm{C}(26)-\mathrm{C}(27)$ 178.5(2)

$\mathrm{S}(3)-\mathrm{N}(3)-\mathrm{C}(27)-\mathrm{C}(28) \quad$ 165.19(15)

$\mathrm{S}(3)-\mathrm{N}(3)-\mathrm{C}(27)-\mathrm{C}(26) \quad 41.7(2)$

$\mathrm{C}(21)-\mathrm{C}(26)-\mathrm{C}(27)-\mathrm{N}(3)-12.7(3)$

$\mathrm{C}(25)-\mathrm{C}(26)-\mathrm{C}(27)-\mathrm{N}(3) \quad$ 169.42(19)

C(21)-C(26)-C(27)-C(28) -134.2(2)

$\mathrm{C}(25)-\mathrm{C}(26)-\mathrm{C}(27)-\mathrm{C}(28)$ 47.9(3)

$\mathrm{N}(3)-\mathrm{C}(27)-\mathrm{C}(28)-\mathrm{C}(29) \quad 122.1(2)$

$\mathrm{C}(26)-\mathrm{C}(27)-\mathrm{C}(28)-\mathrm{C}(29)-114.6(2)$

$\mathrm{C}(27)-\mathrm{C}(28)-\mathrm{C}(29)-\mathrm{C}(30)$ 177.9(2)

$\mathrm{S}(4)-\mathrm{O}(10)-\mathrm{C}(31)-\mathrm{C}(32) \quad 157.47(16)$

$\mathrm{S}(4)-\mathrm{O}(10)-\mathrm{C}(31)-\mathrm{C}(36) \quad-22.9(3)$

$\mathrm{C}(36)-\mathrm{C}(31)-\mathrm{C}(32)-\mathrm{C}(33)$ 2.0(3)

$\mathrm{O}(10)-\mathrm{C}(31)-\mathrm{C}(32)-\mathrm{C}(33)-178.3(2)$

$\mathrm{C}(31)-\mathrm{C}(32)-\mathrm{C}(33)-\mathrm{C}(34) 0.2(4)$

$\mathrm{C}(32)-\mathrm{C}(33)-\mathrm{C}(34)-\mathrm{C}(35)-1.3(4)$

$\mathrm{C}(33)-\mathrm{C}(34)-\mathrm{C}(35)-\mathrm{C}(36)$ 0.4(3)

$\mathrm{C}(32)-\mathrm{C}(31)-\mathrm{C}(36)-\mathrm{C}(35)-2.8(3)$

$\mathrm{O}(10)-\mathrm{C}(31)-\mathrm{C}(36)-\mathrm{C}(35)$ 177.53(19)

$\mathrm{C}(32)-\mathrm{C}(31)-\mathrm{C}(36)-\mathrm{C}(37)-180.0(2)$ 
$\mathrm{O}(10)-\mathrm{C}(31)-\mathrm{C}(36)-\mathrm{C}(37) 0.4(3)$

$\mathrm{C}(34)-\mathrm{C}(35)-\mathrm{C}(36)-\mathrm{C}(31) 1.6(3)$

$\mathrm{C}(34)-\mathrm{C}(35)-\mathrm{C}(36)-\mathrm{C}(37) 178.8(2)$

$\mathrm{S}(4)-\mathrm{N}(4)-\mathrm{C}(37)-\mathrm{C}(38) \quad 169.88(15)$

$\mathrm{S}(4)-\mathrm{N}(4)-\mathrm{C}(37)-\mathrm{C}(36) \quad 43.8(2)$

$\mathrm{C}(31)-\mathrm{C}(36)-\mathrm{C}(37)-\mathrm{N}(4)-11.1(3)$

$\mathrm{C}(35)-\mathrm{C}(36)-\mathrm{C}(37)-\mathrm{N}(4) \quad 171.92(18)$

$\mathrm{C}(31)-\mathrm{C}(36)-\mathrm{C}(37)-\mathrm{C}(38)-135.6(2)$

C(35)-C(36)-C(37)-C(38) 47.3(3)

$\mathrm{N}(4)-\mathrm{C}(37)-\mathrm{C}(38)-\mathrm{C}(39) \quad 3.1(3)$

$\mathrm{C}(36)-\mathrm{C}(37)-\mathrm{C}(38)-\mathrm{C}(39) 128.6(2)$

C(37)-C(38)-C(39)-C(40) 179.4(2)

Symmetry transformations used to generate equivalent atoms:

Table 7. Hydrogen bonds for G114

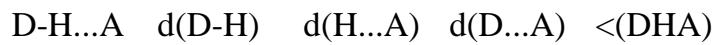

\begin{tabular}{lllll}
\hline $\mathrm{N}(1)-\mathrm{H}(1 \mathrm{~N}) \ldots \mathrm{O}(5) \# 1$ & $0.82(3)$ & $2.19(3)$ & $2.989(2)$ & $168(3)$ \\
$\mathrm{N}(2)-\mathrm{H}(2 \mathrm{~N}) \ldots \mathrm{O}(3) \# 2$ & $0.94(3)$ & $2.07(3)$ & $2.997(3)$ & $168(3)$ \\
$\mathrm{N}(3)-\mathrm{H}(3 \mathrm{~N}) \ldots \mathrm{O}(11) \# 2$ & $0.84(4)$ & $2.15(4)$ & $2.969(3)$ & $166(3)$ \\
$\mathrm{N}(4)-\mathrm{H}(4 \mathrm{~N}) \ldots \mathrm{O}(8) \# 1$ & $0.86(3)$ & $2.13(3)$ & $2.967(2)$ & $164(3)$
\end{tabular}

Symmetry transformations used to generate equivalent atoms:

\#1 $\mathrm{x}+1, \mathrm{y}, \mathrm{z} \quad$ \#2 $\mathrm{x}-1, \mathrm{y}, \mathrm{z}$ 


\section{Reference}

1. Luo, Y.; Carnell, A. J.; Lam, H. W, Angew. Chem. Int. Ed. 2012, 51, 6762-6766.

2. Aikawa, K.; Hioki, Y.; Mikami, K. J. Am. Chem. Soc. 2009, 131, 13922-13923.

3. (a) Toullec, P. Y.; Jagt, R. B. C.; de Vries, J. G.; Feringa, B. L.; Minnaard, A. J. Org. Lett. 2006, 8, 2715. (b)Yin, L.; kanai, M.; Shibasaki, L. Angew. Chem. Int. Ed. 2011, 50, 7620 -7623.

4. Shintani, R.; Inoue, M.; Hayashi, T. Angew. Chem., Int. Ed. 2006, 45, 3353-3356.

5.Kawasaki, T.; Nagaoka, M.; Satoh, T.; Okamoto, A.; Ukon, R.; Ogawa, A. Tetrahedron, 2004, 60, 3493-3503. 